\title{
$Q$ AS A LITHOLOGICAL/HYDROCARBON INDICATOR: FROM FULL WAVEFORM SONIC TO 3D SURFACE SEISMIC FINAL REPORT
}

REPORTING PERIOD: $\quad$ April 29, 2002, - December 31, 2005

SWRI PROJECT MANAGER: Jorge O. Parra, Ph.D., Institute Scientist

TEAM MEMBERS:

C.L. Hackert, L. Wilson, H.A. Collier, J. Todd Thomas

DATE OF ISSUE:

March 31, 2006

DOE CONTRACT NO:

DE-FC26-02NT15343

CONTRACTOR:

Southwest Research Institute ${ }^{\circledR}$ 6220 Culebra Road

San Antonio, Texas 78228-0510

Phone: (210) 522-3284

COST SHARING ORGANIZATION: W.T. Waggoner Estate DOE PROJECT MANAGER: Purna Halder

PROJECT DURATION: Three Years 


\section{DISCLAIMER}

"This report was prepared as an account of work sponsored by an agency of the United States Government. Neither the United States Government nor any agency thereof, nor any of their employees, makes any warranty, express or implied, or assumes any legal liability or responsibility for accuracy, completeness, or usefulness of any information, apparatus, product, or process disclosed, or represents that its use would not infringe privately owned rights. Reference herein to any specific commercial product, process, or service by trade name, trademark, manufacturer, or otherwise does not necessarily constitute or imply its endorsement, recommendation, or favoring by the Unites States Government or any agency thereof. The views and opinions of authors expressed herein do not necessarily state or reflect those of the United States Government or any agency thereof." 


\begin{abstract}
The goal of this project was to develop a method to exploit viscoelastic rock and fluid properties to greatly enhance the sensitivity of surface seismic measurements to the presence of hydrocarbon saturation. To reach the objective, Southwest Research Institute scientists used well log, lithology, production, and 3D seismic data from an oil reservoir located on the Waggoner Ranch in north central Texas.

The project was organized in three phases. In the first phase, we applied modeling techniques to investigate seismic- and acoustic-frequency wave attenuation and its effect on observable wave attributes. We also gathered existing data and acquired new data from the Waggoner Ranch field, so that all needed information was in place for the second phase. During the second phase, we developed methods to extract attenuation from borehole acoustic and surface seismic data. These methods were tested on synthetic data constructed from realistic models and real data. In the third and final phase of the project, we applied this technology to a full data set from the Waggoner site. The results presented in this Final Report show that geological conditions at the site did not allow us to obtain interpretable results from the $Q$ processing algorithm for $3 \mathrm{D}$ seismic data. However, the $Q-\log$ processing algorithm was successfully applied to full waveform sonic data from the Waggoner site.
\end{abstract}

A significant part of this project was technology transfer. We have published several papers and conducted presentations at professional conferences. In particular, we presented the Q-log algorithm and applications at the Society of Exploration Geophysicists (SEG) Development and Production Forum in Austin, Texas, in May 2005. The presentation attracted significant interest from the attendees and, at the request of the SEG delegates, it was placed on the Southwest Research Institute Internet site. The presentation can be obtained from the following link:

http://wWw.swri.org/4org/d15/elecsys/resgeo/ppt/Algorithm.pps

In addition, we presented a second application of the $Q$ algorithm at the SEG International Conference in Houston, Texas, in May 2005. The presentation attracted significant interest there as well, and it can be obtained from the following link:

http://Www.swri.org/4org/d15/elecsys/resgeo/ppt/attenuation.pps 


\section{EXECUTIVE SUMMARY}

Seismic attenuation is a poorly understood physical process that has great potential as an attribute for reservoir characterization. Attenuation is known to be influenced by many critical reservoir parameters, including fracturing, permeability and porosity, the type of saturating fluid, and even fluid viscosity. The development of methods for accurately measuring seismic attenuation is key to exploitation of this attribute for reservoir characterization. In this report, we present and discuss algorithms for measuring seismic attenuation at borehole and field scales, as well as field studies in which attenuation is measured and applied at a Florida aquifer and a north Texas oil field.

At the borehole scale, we developed and tested a processing technique for extracting attenuation information from full waveform sonic logging data. This technique is based on correcting the received head P-wave for effects such as geometrical spreading, multiple reflections, and borehole properties. This method was applied to full-waveform sonic logging data from a south Florida aquifer and a north Texas oil reservoir. In the oil reservoir, an attenuation anomaly was observed at depths that corresponded to known oil saturation. We evaluated several petrophysical models to help interpret the resulting Q logs, including a double-porosity model appropriate for carbonate rocks and an effective medium model for mixed sand-shale sequences.

We also implemented a process for estimating $Q$ from surface seismic data. This process was tested with synthetic data and applied to three real surface seismic data sets from the Florida and north Texas sites. We found that an important complicating factor was spectral tuning resulting from thin-bed interference. We derived a method to correct the observed spectra for the tuning effect in cases where the reflectivity sequence is known, for example from well logs. This correction method was successfully applied to high-resolution surface seismic from the Florida data set. Unfortunately, well control data at the north Texas site was sparse, and we were unable to obtain more than coarse average $Q$ estimates at this site.

Finally, a modeling study was conducted to examine the effects of lateral variability on Q measurement from surface seismic data. Results of this study show that Q measurements are horizontally averaged over a region analogous to the Fresnel zone. However, we also found that abrupt lateral changes in seismic medium properties can introduce diffractions and other waves that closely follow the direct and reflected waves. This often results in interference which manifests itself as spectral distortions. As a consequence, an oscillating error term is often apparent in the measured $Q$ values at or below regions of abrupt lateral change.

We were able to achieve most of our goals for this project, including implementation of methods to successfully extract $Q$ from acoustic logging and surface seismic data. Obtaining accurate $\mathrm{Q}$ values may be difficult in formations with many thin beds, strong lateral variations, or poor well control. As a result of this project, we made ten presentations and published nine articles to share our results with the geophysical community. 


\section{TABLE OF CONTENTS}

ABSTRACT. .ii

EXECUTIVE SUMMARY .iii

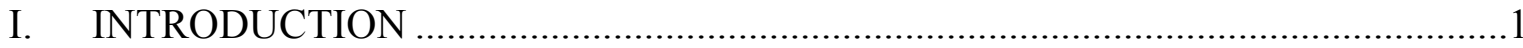

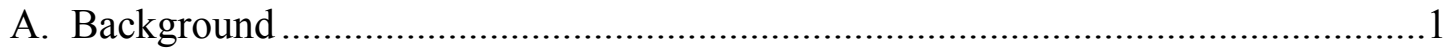

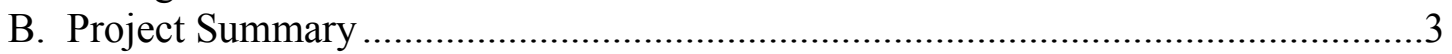

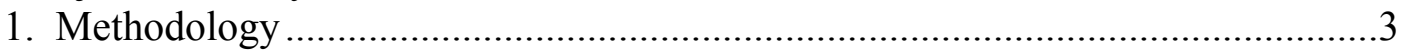

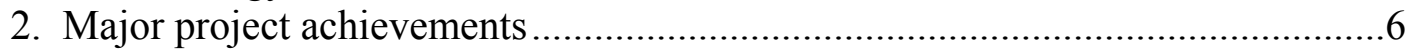

II. ALGORITHM TO EXTRACT Q FROM SONIC LOGS …………..........................

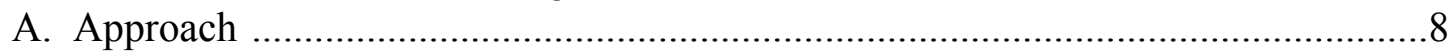

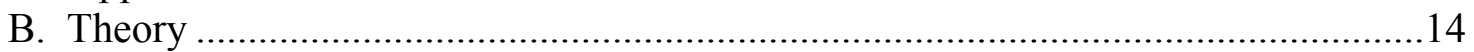

1. The borehole multiple rays model for measured data...........................................14

2. The borehole single ray model for reference data ……......................................17

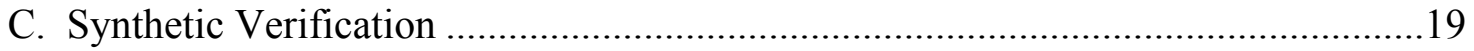

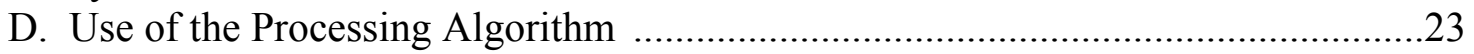

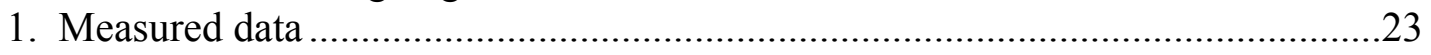

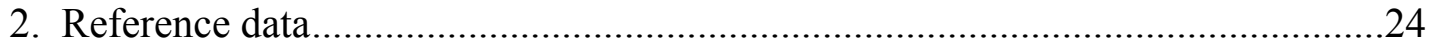

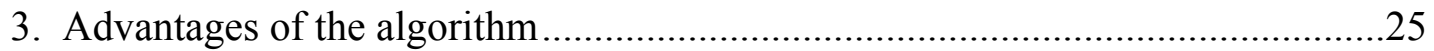

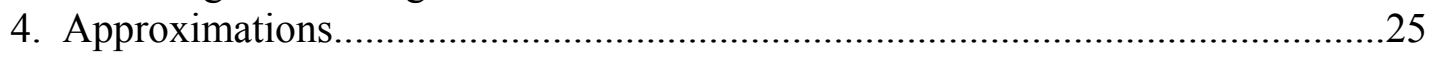

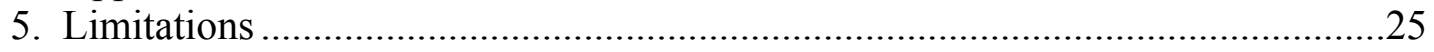

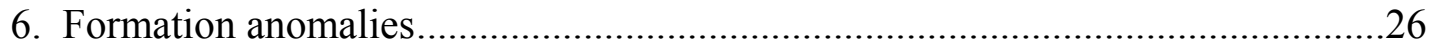

7. Observations based on practical applications ................................................26

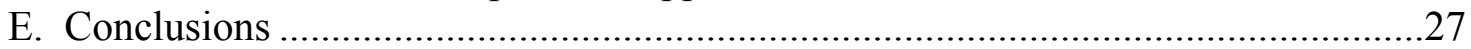

APPENDIX A -BASE FUNCTIONS FOR WAVELET CONSTRUCTION

APPENDIX B - GEOMETRICAL SPREADING OF BOREHOLE HEAD $P$ WAVE

APPENDIX C - REFLECTION AND TRANSMISSION OF WAVESWITH

GEOMETRICAL SPREADING

APPENDIX D - APPLICATION OF ASR METHOD TO A SPECTRAL PEAK

III. WAGGONER RANCH SURFACE GEOLOGY AND PETROPHYSICS …….............34

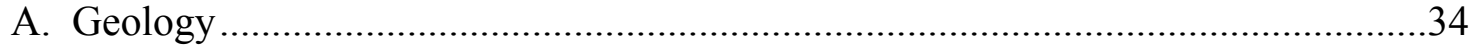

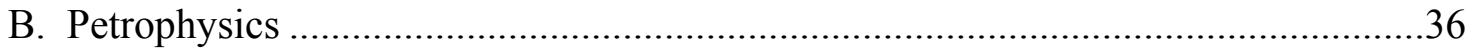

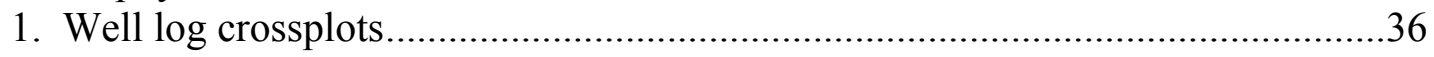

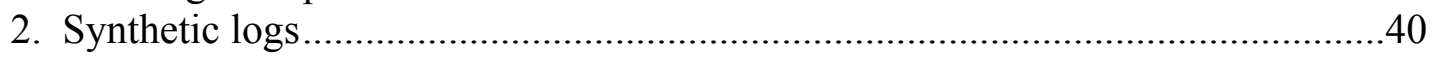

IV. EXTRACTION OF Q LOG FROM WAGGONER DATA …………………..............44

A. Application of the $Q$ Algorithm ...........................................................................45

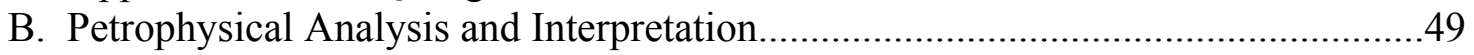

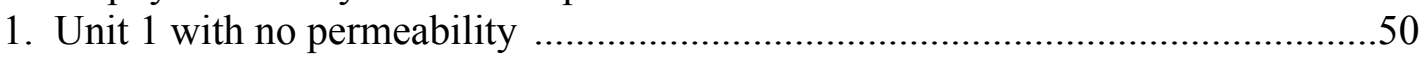

2. Unit 4 with moderate permeability and $15-20 \%$ porosity sandstone .....................51

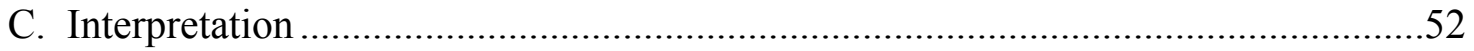

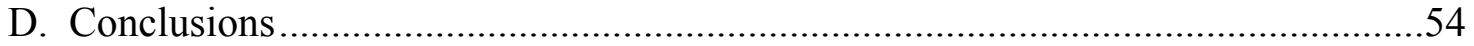




\section{TABLE OF CONTENTS (cont'd)}

V. A DOUBLE-POROSITY POROELASTIC MODEL TO RELATE P-WAVE ATTENUATION TO FLUID FLOW IN VUGGY CARBONATE ROCK ................55

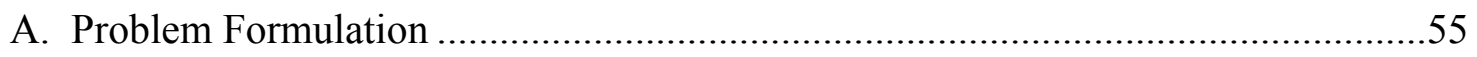

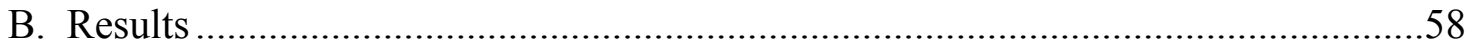

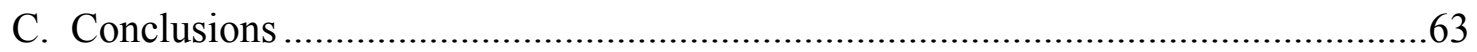

VI. MODELING AND INTERPRETATION OF Q LOGS IN CARBONATE ROCK .....64

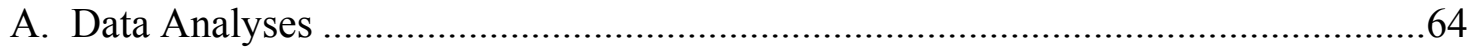

B. Rock Formation Properties and Modeling ..........................................................66

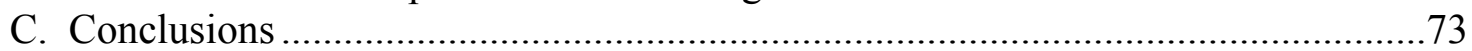

VII. PERMEABILITY AND POROSITY IMAGES BASED ON P-WAVE

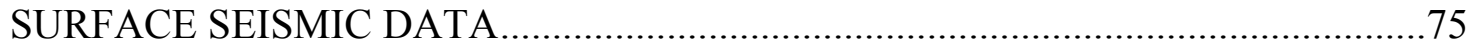

A. Seismic Reflection Method ............................................................................. 76

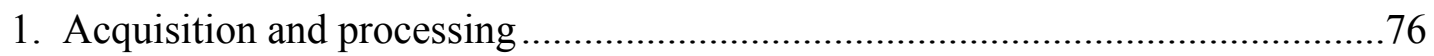

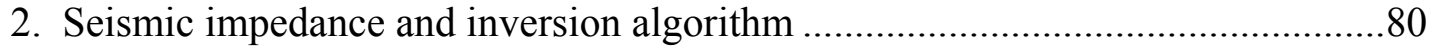

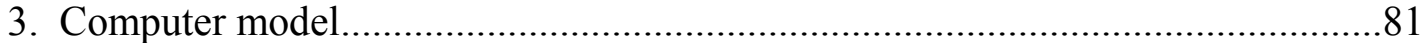

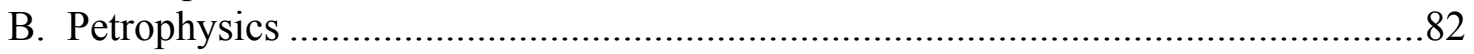

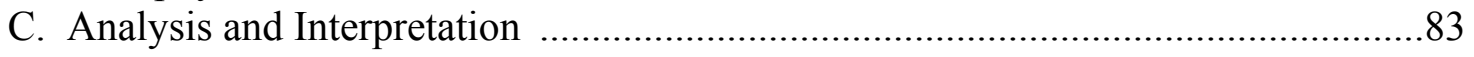

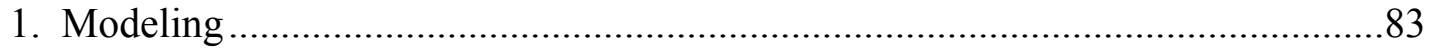

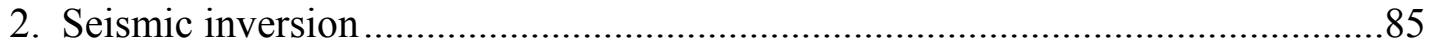

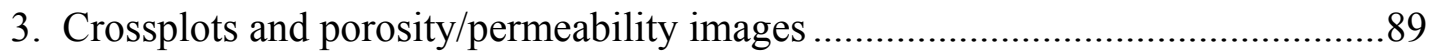

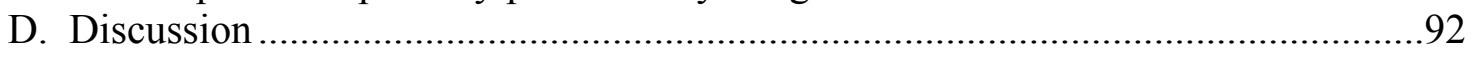

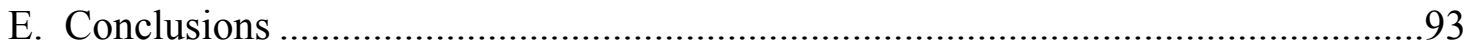

VIII. THEORY FOR ESTIMATING Q FROM SEISMIC REFLECTION DATA .........94

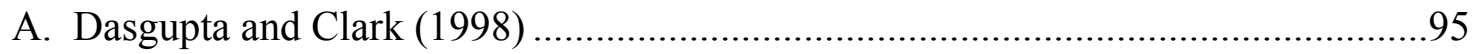

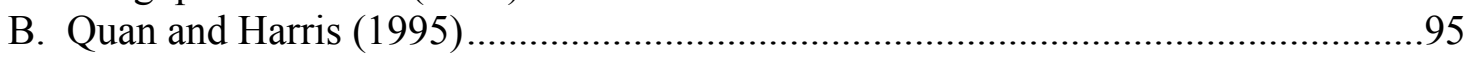

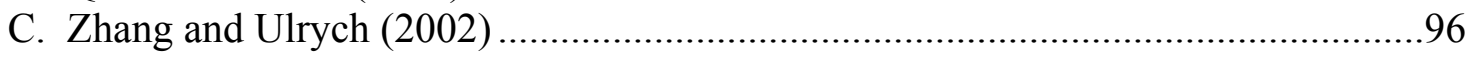

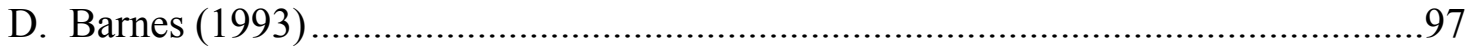

IX. ALGORITHM FOR Q ESTIMATION WITH THIN-BED CORRECTIONS ............98

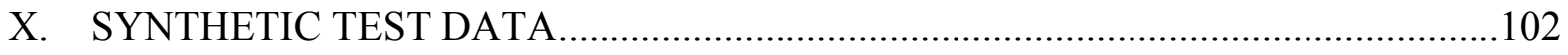

XI. APPLICATION TO FLORIDA SITE (REAL DATA) ......................................... 106

XII. APPLICATION TO WAGGONER SITE (REAL DATA) .....................................111

XIII.EFFECTS OF LATERAL VARIATIONS ON Q MEASUREMENTS $\ldots \ldots \ldots \ldots \ldots \ldots \ldots . . . . . . . . . .116$

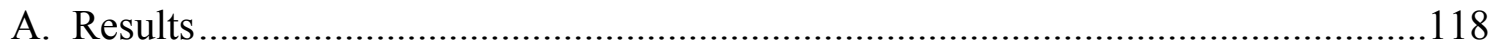

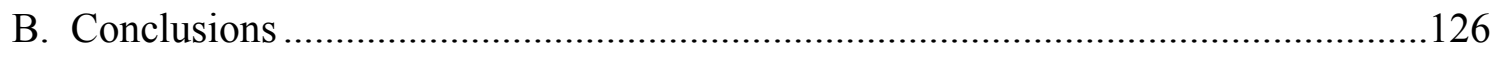




\section{TABLE OF CONTENTS (cont'd)}

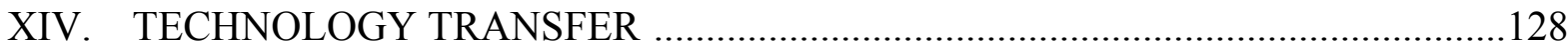

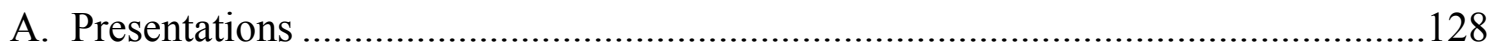

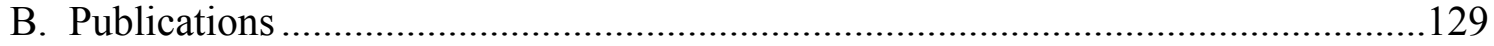

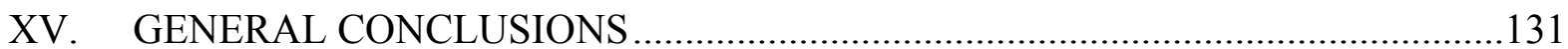

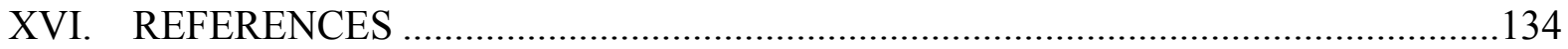


1 Examples of borehole head wave paths. $\mathrm{V}_{\mathrm{P}}$ is the $\mathrm{P}$ wave speed in the formation, $\mathrm{V}_{\mathrm{f}}$ the acoustic wave speed in the fluid, $\mathrm{d}$ the diameter, and $\alpha$ the critical angle.

2 The beginning part of a synthetic full waveform of a borehole sonic $\log$. Model parameters are: $P$ wave velocity $=3 \mathrm{~m} / \mathrm{ms}$, S wave velocity $=1.6$, mass density $=2.25 \mathrm{~g} / \mathrm{cm}^{3}$, borehole diameter $=0.4 \mathrm{~m}(1.3$ $\mathrm{ft}$.), and source-receiver offset $=3.35 \mathrm{~m}$ (11 ft.).

3 Amplitude spectra of synthetic (although labeled as "measured") head $\mathrm{P}$ waves. The center frequency of the source is $10 \mathrm{kHz}$.

4 (a) Amplitude spectrum of borehole head $\mathrm{P}$ wave with a center frequency of $10 \mathrm{kHz}$ at Receiver 1 with offset $=3.35 \mathrm{~m}$ (11 ft.). (b) Same at Receiver 8 with offset $=4.42 \mathrm{~m}$ (14.5 ft.). (c) The geometry and model parameters: P-wave speed $(\mathrm{m} / \mathrm{ms})$, S-wave speed $(\mathrm{m} / \mathrm{ms})$ and mass density $\left(\mathrm{gm} / \mathrm{cm}^{3}\right)$ of materials 1 and 2 are $(4,2,2.7)$ and $(2$, $1,2.4)$, respectively.

5 An example of wavelet fitting of artificial waveform generated by (4).

6 Input (orange) and fitted (blue) waveforms of synthetically generated reference (no attenuation) borehole sonic data at receivers 1 in (a) and 2 in (b).

7 Following Figure 6, (a) shows the extracted first wavelets of "measured" data $\left(\mathrm{Q}_{\mathrm{P}}=40, \mathrm{Q}_{\mathrm{S}}=20\right)$ at receivers 1 and $2(\mathrm{M} 11$-blue; and $\mathrm{M} 21$ - black) and reference data (no attenuation) at receivers 1 and 2 (S11—red, S21—orange); and (b) shows their amplitude spectra.

8 Geometries of the 17 different earth structures for the FD-generated waveforms.

9 Input (blue line) and inverted (red dots) Q for 17 different earth models (see Figure 8).

10 Measured and computed petrophysical properties in lower part of well \#3

11 Crossplots of porosity and permeability with Vp and Vs for shaley and sandstone rock types. 


\section{LIST OF FIGURES(cont'd)}

12 Crossplots of $\mathrm{Q}^{-1}$ with $\mathrm{Vp}, \mathrm{Vs}$, porosity, and permeability for shaley and sandstone rock types.

13 More crossplots from Waggoner well \#3.

14 Comparison of well log Vp and Vs to predicted Vp and Vs after inversion for grain moduli.

15 Comparison of measured and predicted Vp and Vs from well \#3 using grain moduli derived from a test region of 727-762 $\mathrm{m}(2385-2500 \mathrm{ft}$.) in depth.

16 A comparison of measured (black) and predicted (red) Vp in three Waggoner wells, with predictions based on grain moduli derived from depths of 727-762 $\mathrm{m}$ (2385-2500 ft.) in well \#3.

17 Examples of borehole head wave paths. $\mathrm{V}_{\mathrm{P}}$ is the $\mathrm{P}$-wave velocity in the formation, $V_{f}$ is the acoustic wave velocity in the fluid, $d$ is the diameter of the borehole, and $\alpha$ is the critical angle.

18 Location map for wells 1,2 and 3

19 Geological cross section based on lithologic columns from wells 1, 2, and 3 .

20 Comparison between $1 / \mathrm{Q}, \mathrm{V}_{\mathrm{P}}$, and $\mathrm{V}_{\mathrm{S}} \log$ s with acoustic full waveforms and the petrophysics of an oil reservoir.

21 Comparison between acoustic signatures and their spectra in the interval depth of 726-777 $\mathrm{m}(2380-2550 \mathrm{ft}$.).

22 Comparison between standard logs (resistivity, gamma ray, spontaneous potential) and the Q-log to illustrate high attenuation zones versus lithology and fluid saturation.

23 Petrophysical interpretation showing integration of core data and well logs, as well as the seven geological units derived from the core data analysis.

24 Core and thin sections from the interval at 744-747 m (2439-2449 ft.), characterized as low-velocity shale.

25 Core and thin sections from the interval at 759-762 m (2409-2419 ft.), characterized as partially oil-saturated sand. 


\section{LIST OF FIGURES(cont'd)}

26 Core and thin sections from the interval at 759-762 m (2489-2499 ft.), characterized as fully water-saturated sand.

27 (a) Computed density from x-ray CT data, and (b) photograph of the end of the core.

28 (a) Attenuation curves, and (b) pressure spectral response illustrating the length scale effect on attenuation and fluid pressure.

29 Slow-wave pressure spectral responses illustrate the length scale effect on fluid pressure

30 Pressure waveforms illustrating the length scale effect on fast and slow P-wave events. Waveforms correspond to vug sizes of $1,1.5,2$, and 3 $\mathrm{cm}(0.03,0.05,0.066,0.10 \mathrm{ft})$, in a matrix of 3-Darcy permeability.

31 (a) Attenuation curves, and (b) pressure spectral response illustrate the effect of permeability effects on fluid pressure attenuation.

32 Pressure waveforms illustrating permeability effects on fast and slow P-wave events. Waveforms are for $0.5,1,2$, and 3 Darcy permeability, with a vug of radius $\mathrm{a}=2 \mathrm{~cm}(0.066 \mathrm{ft}$. $)$

33 Comparison of sonic waveforms and lithology at the south Florida aquifer

34 Comparison between observed (gray line) and predicted (black line) attenuation logs with the permeability, vuggy porosity, and matrix porosity logs.

35 Crossplots of P-wave and S-wave velocity logs and density log, with the total porosity log in percent.

36 Comparison of matrix bulk and shear modulus with the vug space bulk modulus.

37 Attenuation curves illustrating the length scale effects on the attenuation associated with the vuggy carbonate at a depth of $335 \mathrm{~m}$ (1100 ft.).

38 Attenuation curves illustrating the length scale effects on the attenuation associated with the chalky carbonate at a depth of $357 \mathrm{~m}$ (1170 ft.). 


\section{LIST OF FIGURES(cont'd)}

39 Comparison between observed (gray line) and calculated (heavy line) attenuation logs with the lithology at the south Florida aquifer.

40 (a) ASR Pilot Project regional map; (b) location of seismic experiments at the western Hillsboro Aquifer Storage Recovery (ASR) site near Boca Raton, Florida.

41 Shot gather recorded at station 179.5.

42 Observed surface seismic reflection data illustrated as a stacked CDP section.

43 Well logs from well PBF10, south Florida. Comparison of Vp, Vs, density, and gamma ray log with lithology, Q log, permeability and porosity.

44 A comparison of full waveform monopole sonic data with the lithological column between $229 \mathrm{~m}$ (750 ft.) and $381 \mathrm{~m}(1250 \mathrm{ft}$.).

45 2D surface seismic reflection data.

46 P-wave impedance from south Florida 2D surface seismic data.

47 Comparison of well log impedance (black) from the PBF10 well and impedance from the surface seismic line at the same location (red).

48 A section of the P-impedance from surface seismic data, which overlaps with the crosswell reflection data.

49 P-wave impedance computed from crosswell reflection data.

50 (a) Crossplot of well log impedance with well log NMR permeability from a $290-\mathrm{m}(950-\mathrm{ft}$.) to a 381-m (1250-ft.) depth, and corresponding fit equation; (b) Crossplot of well log impedance with well log porosity from a 290-m (950-ft.) to a 381-m (1250-ft.) depth, and corresponding fit equation.

51 (a) Comparison between the NMR-permeability well log (green) and the permeability (red) derived from regression Equation (6); (b) Comparison between the porosity well log (green) and porosity (red) derived from regression Equation (7). 


\section{LIST OF FIGURES(cont'd)}

52 Permeability (top) and porosity (bottom) inferred from surface seismic

P-impedance and correlations with well logs.

53 This figure shows the permeability image (top) and porosity image (bottom) computed from the P-wave impedance derived from crosswell seismic data.

54 Three hypothetical spike reflectivity stickogram sequences and their corresponding spectra.

55 Models for synthetic seismic reflection data without and with thin beds added near each main layer boundary.

56 Seismic reflection response of synthetic models without (left) and with (right) thin beds near each main layer boundary.

57 Comparison between uncorrected and corrected normalized local reflection spectra.

58 Migrated seismic data and well logs from the Florida aquifer test site.

59 An unmuted pre-stack CDP NMO gather from the South Florida surface seismic data set.

60 (top) Plots of corrected and uncorrected log-spectral-ratio as a function of frequency for CDP 128, offset $1111 \mathrm{ft}$. in the Florida data. (bottom) $\mathrm{Q}^{-1}$ versus offset plots for CDP 128 of log-spectral-ratio $\mathrm{Q}^{-1}$ (square symbols with error bars) and centroid-frequency $\mathrm{Q}^{-1}$ (triangles).

61 Comparison of uncorrected (left) and corrected (right) $\mathrm{Q}^{-1}$ values computed from two sets of reflections from the Florida seismic line.

62 Cutaway view of 3-D extension survey impedance

63 Map of $\mathrm{Q}^{-1}$ computed via the centroid frequency QVO method from the Waggoner extension shoot.

64 Impedance volume image of Waggoner Ranch 3D seismic data.

$65 \mathrm{Q}$ values resulting from processing of the Waggoner 3D seismic data.

66 Comparison of mean structural impedance and recovered spectral ratio $\mathrm{Q}$ values for comparable time horizons. 
67 Comparison of mean structural impedance and recovered centroid frequency shift $\mathrm{Q}$ values for comparable time horizons.

68 Test geometry. The middle layer consists of wet sand, fizz sand, and gas sand.

69 Processed and CMP-stacked data based on model of Table 11

70 Plot of log-spectral ratios of three traces, from reflections 1 to 3 , at different CMP locations.

71 Lateral variation of apparent $\mathrm{Q}^{-1}$ : (a) from base of shale 1 to base of shale 2 (top sand), (b) from base of shale 1 to base of sand layer, and (c) from base of shale 1 to base of shale 3 .

72 Snapshots of vertical particle velocity at different times, with the source at $x=1200 \mathrm{~m}(3936 \mathrm{ft})$. Red indicates positive vertical velocity, and blue indicates negative vertical velocity.

$73 \mathrm{Q}^{-1}$ versus offset-squared for three CMP gathers at the base sand reflector, showing normal, suppressed, and enhanced attenuation. 


\section{LIST OF TABLES}

Table 1. Input Parameters for the Synthetic Borehole Head $P$ Waves Used in

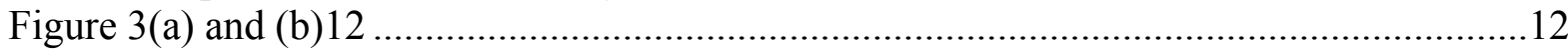

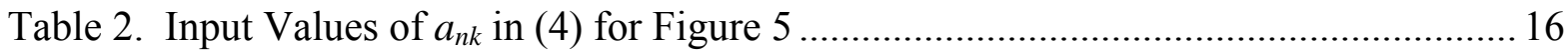

Table 3. $P$-, $S$-wave Speeds $(\mathrm{km} / \mathrm{s})$ and Mass Density $\left(\mathrm{gm} / \mathrm{cm}^{3}\right)$ in Earth Structures .......... 19

Table 4. Source-receiver Offsets.............................................................................. 20

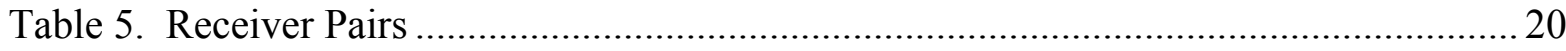

Table 6. Inverted and Typical Grain Moduli for Waggoner Rock Constituents .................. 41

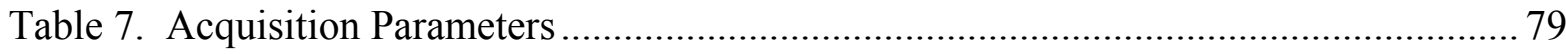

Table 8. True and Computed Values of $Q$ from the Synthetic Test Data......................... 103

Table 9. Effect of Well Log Error on Uncertainty of Computed, Spectrally

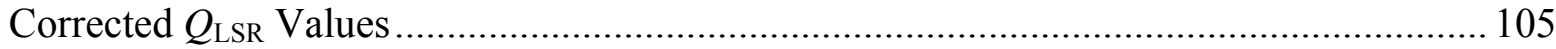

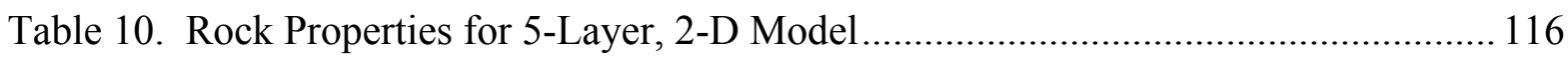

Table 11. True and Estimated Q Values for Laterally Homogeneous Cases..................... 118 


\section{INTRODUCTION}

\section{A. Background}

Seismic attenuation is a physical process governing the reduction in amplitude of seismic energy as it propagates through the ground. It is caused by a number of physical mechanisms, some of which are poorly understood. These include viscous and viscoelastic wave damping by internal frictional forces in the rock and its saturating fluid, as well as wave scattering at lithological boundaries and fractures or joints. The influence of rock pore structure and saturating fluid on attenuation give some hope that attenuation may be useful as an indicator for various reservoir properties. At the same time, understanding the effect of attenuation on seismic wave propagation can lead to processing for improved surface seismic images. Since seismic amplitude measurements are also affected by other parameters including geometric spreading and rock stiffness, measurements of seismic attenuation are most commonly performed using the spectral ratio method. This method exploits the fact that higher frequencies in seismic waves tend to attenuate more rapidly because of their smaller wavelength. The spectral ratio method has been fairly successful in extracting attenuation values from transmitted seismic data such as that recorded in vertical seismic profiles or crosswell experiments, but has had less success in the analysis of seismic reflection data, because seismic reflection data is more strongly affected by the presence of closely spaced layers in the rock whose combined reflections can appear to change the spectral content of the wave.

Acoustic borehole wave attenuation is a powerful attribute that can be used as an indicator of lithology, pore structure, fractures, and clay and fluid content in a reservoir characterization program. Since acoustic attributes from full waveform sonic logs can be easily related to petrophysics and core data in a single borehole, the intrinsic and scattering effects at the borehole scale can be determined. Since the early 1980s, various researchers have attempted to develop techniques to determine $Q$ from single-hole full waveform acoustic $\log$ data. It is, however, commonly recognized that existing $Q$ processing techniques have major limitations and pitfalls. In particular, these techniques encounter serious difficulties when the lithology and acoustic properties are highly heterogeneous in the depth direction. Additionally, in the sonic signals, the influence of formation heterogeneity is coupled with the interaction among internally reflected rays of the borehole head wave.

Our goal was to develop a method to exploit viscoelastic rock and fluid properties to greatly enhance the sensitivity of surface seismic measurements to the presence of hydrocarbon saturation. We used well log, lithology, production, and 3D seismic data from an oil reservoir on the Waggoner Ranch in north central Texas. The program resulted in a methodology for processing and interpreting surface/subsurface data to determine reservoir properties and for applying that information to predict the presence of hydrocarbons as well as volume estimates in complex geological environments.

As seismic data analysis becomes more advanced, interpreters now examine the smallest details of the seismic record. To extract more and more useful information from seismic data, geophysicists have developed techniques to look not only at the sequence of reflections, but also bright spots, amplitude variation with offset and azimuth (AVO/AVA), and even instantaneous attributes such as frequency and phase. It seems 
likely that knowledge of $Q$, the quality factor of the medium, will one day be important for providing additional information about the formation. In addition to the well-known features of gas and fractures being associated with low $Q$ (high attenuation of seismic waves), a number of recent works have proposed using $Q$ as an indicator of reservoir quality (Parra and Hackert, 2002; Pride et al., 2003; Rapoport et al., 2004; Korneev et al., 2004) and as a numerical basis for 'inverse filtering' (Zhang and Ulrych, 2002). Inverse filtering involves raising the high-frequency content of later times in seismic sections to compensate for seismic wave attenuation and to improve image resolution.

Several projects have demonstrated the calculation of $Q$ from seismic transmission data such as VSP (Hauge, 1981), crosswell (Quan and Harris, 1995; Neep et al., 1996), and sonic logging (Parra and Hackert, 2006). Very few publications, however, have addressed computing $Q$ from reflection data, and even fewer have published $Q$ value estimates from real surface seismic data. Dasgupta and Clark (1998) is a notable exception. Part of the reason for this is the complexity of surface seismic data. Compared to transmission signals, reflection signals have a longer path length, since they must travel back to the surface. The amplitude is further reduced by the reflection coefficient of the layer of interest (usually less than 0.1). A single stacked common depth point (CDP) reflection event comprises signals from multiple offsets, each representing a different travel path, path length, angle of incidence, and source-receiver pair. The short travel times and narrow bandwidth of seismic pulses often combine with interfering reflections from thin beds to defeat the spectrum-based methods most commonly used to estimate attenuation. Nevertheless, surface seismic data is so useful and common that it is worthwhile to attempt to recover $Q$ values from this source. In some cases, the limitations can be overcome by using high-frequency (wide-bandwidth) sources, estimating average $Q$ over large depth ranges, restricting analysis to reservoirs with simple geology, and using well control to remove spectral distortion in post-processing (Hackert and Parra, 2004).

Furthermore, while vertical variability and layering rightly capture a great deal of attention, resolution of lateral variations in $Q$ was cited as a source of uncertainty at the 2005 SEG Development and Production Forum on Seismic Attenuation. Few researchers have treated this problem. Adriansyah and McMechan (1998) examine the effects of a laterally variable media, including variable $Q$ on AVO measurements. Dasgupta and Clark (1998) show evidence of a decrease in $Q$ measured laterally across a gas-charged reservoir in the North Sea. However, the boundaries of the event are not clear and it is uncertain to what extent this ambiguity arises from gradual horizontal changes in $Q$ or inherent resolution issues in long-offset seismic processing.

In this report, we describe the theory and the development of a method to extract $Q$ logs from full waveform sonic data, and the implementation of a second method for estimating $Q$ from surface seismic reflection data based on that of Dasgupta and Clark (1998). The surface seismic reflection method was extended to include corrections for known thin-bed layering. After both methods (the $Q \log$ and surface methods) were tested with synthetic data, we applied them to process real datasets from a south Florida aquifer and a north Texas oil field. In particular, we applied the $Q$ estimation method to synthetic surface seismic data, to study the effects of lateral variation in seismic properties on $Q$ measurements. Our results indicate that $Q$ estimation from surface seismic data can be successfully applied, but many factors (including vertical and 
horizontal inhomogeneity, low seismic frequencies, thin reservoir units, and poor well control) can negatively impact $Q$ measurements.

\section{B. Project Summary}

The project was organized in three phases. In the first phase (year I), we applied modeling techniques to investigate seismic- and acoustic-frequency wave attenuation and their effect on observable wave attributes. We also gathered existing data and acquired new data from the Waggoner Ranch field. During the second phase (year II), we used this information to develop methods to extract attenuation $Q$ values from borehole acoustic and surface seismic data. These two methods, briefly described below, were tested on synthetic data constructed from realistic models and real data. In the third phase (year III), we applied and verified the $Q$-inversion method with sonic data from south Florida and we processed data from the Waggoner Ranch. Work completed under each phase is presented as follows: data gathering, experimental methods/technical approach, and algorithm development are reported first; and results and discussion/conclusions are reported last.

The report is organized primarily into two comprehensive discussion areas. The first area includes the theory, development, and applications of the algorithm to extract $Q$ from sonic logs, as well as the geology of the Waggoner Ranch site, and it is contained in Sections II-VIII. The second area concerns the theory, development, and applications of the algorithm to extract $Q$ from surface seismic data, and it is contained in Sections IX-

XIII. Section XIV describes technology transfer accomplishments, and Section XV presents our overall conclusions.

\section{Methodology}

\section{Algorithm to extract $Q$ from sonic logs}

A processing algorithm to extract $Q$ from full waveform sonic logs was developed and tested with synthetic data. The algorithm is original because it corrects the apparent attenuation for the mixed effect of multiple borehole rays, geometrical spreading of head waves, and formation inhomogeneity. The algorithm makes use of the head $\mathrm{P}$ wave of the sonic log and takes into account the vertical conditions (e.g., thin layers, boundaries, etc.) penetrating the borehole. The processing algorithm includes two ray borehole models to invert the acoustic signal for Q. The first model is a multiple ray solution that simulates the interaction between internally reflected rays within the borehole and reflections from layer interfaces. The model is applied to extract the raw signal, the leading borehole wavelet from both internal and external reflections. Thus, the corrected measured data at two receiver locations for these effects is obtained. The second model is a borehole single ray model in layered formations. This ray model provides the transmission coefficients across layer interfaces of the formation surrounding the borehole. As a result the ray undergoes geometrical spreading layer by layer on its path. Both models allow us to separate, correct for, and normalize the effects of the borehole, geometrical spreading, and layering at the same time. We tested the accuracy and limits 
of the model with synthetic data generated using the solution of the point source in a fluid-filled borehole surrounded by an unbounded medium, which is the classic wave number integral method. The relative error of $Q$ was found to be within 5 percent. A second set of tests was performed on synthetic full waveform sonic data logs in uniform and layered formations generated by finite difference models. In general, agreement was very good when both receivers were in the same layer or half space. Agreement was fair when the two receivers crossed an interface. In Section II, we describe concepts, theory and testing of the $Q$ algorithm to process sonic logs.

We applied the processing algorithm to extract $Q$ logs from two data sets - one from a sonic log acquired in the oil reservoir in north Texas, and the second from a sonic $\log$ acquired in the south Florida aquifer. The Texas site is a sand-shale sequence oil reservoir, which the attenuation log correlates with the lithology, including a sandstone zone partially saturated with oil and water. The geology and the petrophysics of this site are described in Section III. We found that the $Q$ log detected a zone that contains oil and water, which suggested fluid motion between the oil and water in the presence of a gas phase. This reduced the acoustic energy. In addition, with the help of petrophysics, the $Q$ log allowed us to evaluate acoustic attributes in the microseismogram. This application is described in Section IV.

To explain the wave attenuation associated with rock/fluid types of a reservoir, several self-consistent and empirical models for intrinsic and scattering attenuation were evaluated. A double porosity model was effective to evaluate the $Q \log$ processing algorithm. The theory and the model responses of the double porosity carbonate rock are given in Section V. The double porosity model was generalized to simulate $Q$ logs (i.e., $Q$ as a function of depth). In the Florida aquifer we evaluated the attenuation log using porosity logs, rock physical properties, and the double porosity model. We also used vuggy and matrix porosity logs obtained by a joint inversion of resistivity and velocity logs. We integrated the porosity logs with the lithology to visualize a region that contains different facies that give different acoustic signatures. The description of this application is given in Section VI, which also section describes a complete verification of the $Q$ algorithm with real sonic data using the double porosity model and the petrophysics that support the results.

As a result of the work done in the carbonate aquifer, we integrated the south Florida data sets to demonstrate a practical application that allowed us to map permeability and porosity at field scales using acoustic and seismic data with petrophysics. Section VII describes the use of P-wave surface seismic reflection data to delineate flow units in a proposed Aquifer Storage and Recovery (ASR) horizon. The impedance image determined by inversion from P-wave reflection data captures most of the boundaries between hydraulic facies. High-resolution porosity, permeability, and impedance images based on crosswell reflection and well logs helped us evaluate the Pwave seismic reflection results. We use well logs and crosswell seismic data to support whether 2D seismic reflection measurements detect important flow units delineated by crosswell high-resolution seismic data. This work allowed us to produce porosity and permeability images of the carbonate aquifer. 


\section{Algorithm to extract $Q$ from surface seismic data}

The method for estimating $Q$ from surface seismic data is based on the $Q$-versusoffset method of Dasgupta and Clark (1998), and estimates $Q$ using the spectral ratio or frequency-shift method by comparing reflection spectra from multiple offsets in each CMP gather. We tested the algorithm with synthetic data and applied it to three real surface seismic data sets from two sites. While the overall method was successful, we found a number of complicating factors can interfere with obtaining useful $Q$ measurements. These factors include thin-bed reflections, lateral variability in seismic properties, and low bandwidth or excessive noise in the seismic data. Some of these factors can be overcome in post-processing.

In Section VIII, we review some of the theory behind estimating $Q$ from seismic reflection data. The basic, primary methods used here are the spectral ratio method, which estimates $Q$ by the relative attenuation of high and low frequencies, and the frequency-shift method, which estimates $Q$ by the shift of the spectrum centroid. Both methods require comparing a reflection spectrum with a reference spectrum, usually from another reflection. The resulting $Q$ is an average from the depth of the reference measurement to the depth of the reflection.

In Section IX, we present a method for correcting reflection spectra with distortions caused by multiple interfering reflections caused by thin beds. This spectral distortion, an example of the well-known "tuning" effect in thin-bed reflections, can obstruct accurate $Q$ estimation. This is because $Q$ estimation techniques rely on clean, accurate spectra in order to work well. By using the known thin-bed reflection sequence from well logs, the observed spectra may be corrected to remove the thin-bed effects. If the well logs have a sufficiently good tie to the seismic data, the resulting corrected spectra are much better for use in Q-estimation techniques. This is demonstrated in Section $\mathrm{X}$, where we apply the thin-bed correction and $Q$ estimation algorithm to synthetic data generated from a finite-difference model. The algorithm is verified by recovering the input model parameters.

We apply the same method in Section XI to real data, a high-resolution 2-D seismic line over a Florida aquifer. This was a good test site for our study, because there was an extensive suite of logs and tests at this location. Without the thin-bed correction algorithm, the measured $Q$ profile showed a large degree of variability and included many unphysical negative $Q$ values. With the thin-bed spectral corrections, the measured $Q$ was near 33 across the line using both the spectral-ratio and frequency-shift methods.

In Section XII, we apply our method to two 3D seismic data sets from the north Texas oil field. These data sets covered a greater spatial extent with fewer offsets per CDP than the Florida data, and used a lower bandwidth. We were unable to apply the thin-bed correction technique because only a few wells had the necessary logs, and these generally did not tie well to the seismic data. The resulting $Q$ maps had very high variability, with a large number of negative or excessively high $Q^{-1}$ values. The overall average $Q$ for the field was in the range of 25-50, which is reasonable, but we could not identify any trends that correlated with known structures or oil production.

Finally, in Section XII, we provide a modeling study of the effects of lateral variation in seismic properties on $Q$ measurement. As expected, we find that measured $Q$ values are spatially averaged over a horizontal region that increases with depth, 
analogous to the Fresnel zone. A more interesting and important result, however, is that lateral changes in seismic properties produce diffractions, converted waves, and interface waves that can produce interference that distorts the reflected wave at the common mid point (CMP) of the lateral boundary. Like the thin-bed effects discussed earlier, any interference in the reflected wave will obstruct accurate $Q$ estimation. As some of these interfering waves propagate downward, reflections from lower strata may also the distorted at the CMP of the lateral change.

\section{Major project achievements}

- Development of an acoustic $Q$ inversion technique to extract attenuation from full waveform sonic logs. The method was successfully tested and verified with sonic data acquired in a vuggy carbonate formation using a double-porosity model. The technique was also applied to sonic data from the Waggoner reservoir, where the $Q \log$ captured the presence of oil saturation.

- Development and implementation of an algorithm for correcting the spectral content of seismic reflections for the presence of closely-spaced reflectors in order to accurately extract attenuation information. In this process, the spacing and properties of these reflectors must be known (from well logs) so that a convolutional model of the resulting spectral distortion can be computed. By correcting the observed spectra using the model spectral distortion, we obtain an accurate representation of the seismic wave spectrum, which can then be analyzed with the spectral ratio method to compute attenuation $Q$ factors. 


\section{ALGORITHM TO EXTRACT Q FROM SONIC LOGS}

Seismic attenuation is a powerful attribute that can be used as an indicator of lithology, pore structure, fractures, and clay and fluid content in a reservoir characterization program. Acoustic attributes from full waveform sonic logs effectively relate to petrophysics and core data in a single borehole. The intrinsic and scattering effects at the borehole scale can therefore be determined. Since the early 1980s, many researchers have attempted to develop techniques for the inversion of $Q_{P}$ from singlehole full waveform acoustic log data; in particular, to obtain depth profiles of intrinsic $Q_{P}{ }^{-1}$ from the head $P$ wave of the sonic data. In a popular approach, $Q_{P}{ }^{-1}$ is calculated from the Amplitude Spectral Ratio (ASR) of the head $P$ wave measured at two locations (e.g., Cheng et al., 1982; Sun et al., 2000; Dasios et al., 2004). In this approach, the borehole head wave is analogous to a plane wave in a uniform unbounded medium. The degree of success of existing work on $Q_{P}{ }^{-1}$ varies, but in general is not very satisfactory. In our view, the key to success is to be able to fully understand the signal of the head $P$ wave, and to quantify and account for other factors besides $Q_{P}{ }^{-1}$ that alter the amplitude of recorded signals.

The first of such factors is the heterogeneity of rocks in terms of acoustic impedance, as well as the associated wave reflections and scattering from fractures and discontinuities. Changes in acoustic impedance cause general changes in wave amplitude, regardless of attenuation. In addition, reflections and scattering introduce oscillations to the amplitude spectra, which make the processing of $Q_{P}{ }^{-1}$ more difficult. Observing data from many cases in the literature, it is evident that reflections cause significant amplitude change and are responsible for the poor results of $Q_{P}^{-1}$. Some but not all authors of those results recognize the adverse effect of reflections on $Q_{P}{ }^{-1}$ processing and employed numerical and statistical methods to suppress the effect of reflections. However, no quality models based on the physics of the reflection have been attempted. In this sense, none of the existing $Q_{P}{ }^{-1}$ processing techniques is capable of providing reliable results for heterogeneous formations.

The second factor is the geometrical spreading of head waves, which reduces wave amplitude in addition to attenuation. In slow formations, the leaky wave arrivals further complicate the geometrical spreading. Geometrical spreading is ignored in some existing processing techniques.

The third factor is the interaction among multiple rays of borehole head waves with different arrival times, which result from progressive reflections within the borehole. These multiple arrivals form a continuous head wave train, which manifests itself as multiple peaks with different amplitudes in the amplitude spectrum. When different formation properties are encountered on the path reaching the receiver, the amplitudes of some peaks are significantly modified while the amplitudes of other peaks are not. Such coupling of the borehole geometry and formation heterogeneity is in effect even when there are no reflections from the formation interfaces in the gated signal. Therefore, coupling between the borehole effect and formation heterogeneity makes a direct application of ASR difficult. No correction attempts for this aspect of the problem were reported in the literature.

In this section, we describe a processing algorithm to obtain depth profiles of intrinsic $Q$ from full waveform borehole sonic logs. The processing technique makes use 
of the head $P$ wave of the sonic log and takes into account the inhomogeneity (e.g., thin layers, boundaries) conditions of the formations in the vertical direction along the borehole. Coupled effects of inhomogeneity and the borehole are identified and applied for correcting the signal. We implement two different but related ray models to analyze and invert sonic signals for extracting intrinsic attenuation. One is a modified ray tracer for layered formations, which takes into account geometrical spreading of the head wave. The other is a borehole ray model where the interaction among internally reflected rays with different arrival times is considered. We are able to separate, correct for, and normalize the effects of the borehole, geometrical spreading, and layering simultaneously. Finally, to test the algorithm for accuracy we use synthetic data based on analytical and finite difference models, and we calculate $Q$ at each depth with the ASR in properly defined peak areas of the corrected and normalized spectrum.

\section{A. Approach}

Our $Q_{P}$ processing technique is based on an extended version of the ASR method. We make use of the head $P$ wave of the sonic log, but the technique can be applied to head $S$ wave and guided waves. In the ASR method, the $P$ wave attenuation $Q_{P}{ }^{-1}$ is given by

$Q_{P}^{-1}=\frac{2 V_{P}}{\omega\left(z_{2}-z_{1}\right)}\left[\ln \frac{M_{1}(\omega)}{M_{2}(\omega)}-\ln \frac{S_{1}(\omega)}{S_{2}(\omega)}\right]$

This equation can also be found in Toksoz et al (1979) and Cheng et al. (1982), in a slightly different form. In these two papers, $M_{1}$ and $M_{2}$ denote the amplitudes of the seismic waves from a common source measured at distances $z_{1}$ and $z_{2}$, respectively. $S_{1}$ and $S_{2}$ represent the geometrical factor such as geometrical spreading and reflections at these two receivers. In the absence of attenuation, $M_{1}$ and $M_{2}$ equal $S_{1}$ and $S_{2}$, respectively.

A major difficulty associated with this method is the identification and separation of the mixed effects of multiple borehole rays, geometrical spreading and formation inhomogeneity on the amplitude change. To account for these mixed effects, we express the intrinsic attenuation of head $P$ wave, $1 / Q_{P}$, as

$$
\frac{1}{Q_{P}}=\frac{1}{Q_{0}}-\left(\frac{1}{Q_{1}}+\frac{1}{Q_{2}}+\frac{1}{Q_{3 a}}+\frac{1}{Q_{3 b}}\right)
$$

where $1 / Q_{0}$ represents the apparent attenuation from the raw sonic signal at two receivers, and $\left(1 / Q_{1}+1 / Q_{2}+1 / Q_{3 a}+1 / Q_{3 b}\right)$ represent the correction term depending on the geometry of the source, borehole and surrounding layered medium. Among them, $1 / Q_{1}$ is due to the interaction among multiple borehole internal rays, $1 / Q_{2}$ due to geometrical spreading from the source, $1 / Q_{3 a}$ due to frequency independent transmission loss or gain at layer interfaces, and $1 / Q_{3 b}$ due to reflections in the layers. These four terms are coupled. 
A natural approach would be taking $1 / Q_{0}$ in (2) as the term of logarithmic $M_{1} / M_{2}$ in Equation (1), and the grouped four terms in Equation (2) as the term of logarithmic $S_{1} / S_{2}$ in Equation (1). In doing so, one would rely on synthetic full wave solutions for borehole waves in layered media to obtain $S_{1} / S_{2}$. For example, solutions provided by the boundary element (BE) model (e.g., Bouchon, 1993) or the finite difference (FD) model can be used. However, there are two problems with such an approach. First, an algorithm directly derived from the BE or FD models would be rather inefficient for processing a large volume of field data on general purpose computers or workstations. Second, the amplitude spectra of so defined $M_{1}, M_{2}, S_{1}$ and $S_{2}$ typically have very irregular shapes, which make the application of Equation (1) difficult.

In the following, we compare the shapes of amplitude spectra of waves for different geometries of source and propagation media and examine how they affect the use of Equation (1). We assume that the source function is a single wavelet with a center frequency of $f_{c}$ and duration $\tau=2 / f_{c}$. Its amplitude spectrum has a single peak at $f_{c}$.

First consider Equation (1) for the plane wave, which is non-dispersive and has no geometrical spreading, i.e. $S_{1}=S_{2}$. In this case, the full waveform recorded at any receiver remains a single wavelet and the frequency content remains the same as that at the source. Next, for waves with geometrical spreading but no reflections, such as those from a point or line source in uniform unbounded media, the full wave form is again a single wavelet, and the frequency content of the spread wave also remains the same as that of the source wavelet. In this case, the logarithmic $S_{1} / S_{2}$ does correct for the geometrical spreading. Therefore the application of Equation (1) is again straightforward.

The borehole head wave in uniform media is quite different from the above two cases. In addition to geometrical spreading, there are multiple arrivals due to internal reflections. Figure 1 shows the primary (or first) ray and some subsequent rays in the borehole. Let $\delta$ denote the time delay unit between any adjacent rays. It can be seen that the head wave consists of a series of wavelets with periodical delays measured by multiples of $\delta$. When $\delta>\tau=2 / f_{c}$, the wavelets are completely separated in time as seen in Figure 2. Otherwise, they are all overlapped.

In addition, the amplitude spectrum of the head wave has multiple peaks. Its maximum peak is no longer at $f_{c}$. Figure 3 shows $M_{1}$ and $M_{2}$ equal $S_{1}$ and $S_{2}$ for two sets of model parameters. Note that the shapes of the maximum peak of all four amplitude spectra are very similar. Our experience shows that applying Equation (1) to the first major peak yield quite accurate result of $1 / Q_{P}$. 


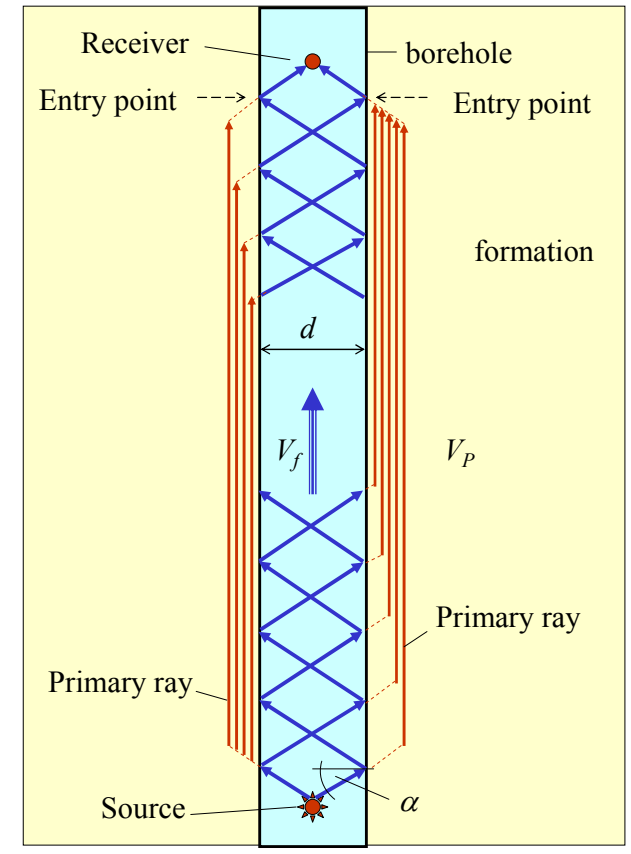

Figure 1. Examples of borehole head wave paths. $V_{P}$ is the $P$ wave speed in the formation, $V_{f}$ the acoustic wave speed in the fluid, $d$ the diameter, and $\alpha$ the critical angle. All wave fronts are cylindrically symmetric about the borehole axis. However, to show more possible paths, we allow the left and right ones to be different.

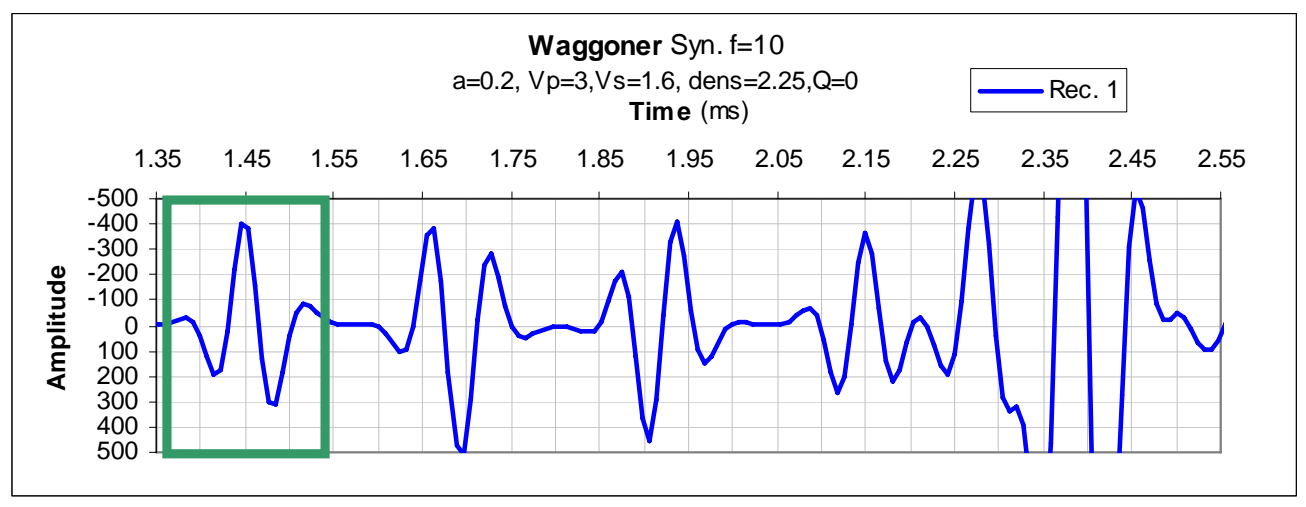

Figure 2. The beginning part of a synthetic full waveform of a borehole sonic log. Model parameters are: $P$ wave velocity $=3 \mathrm{~m} / \mathrm{ms}, S$ wave velocity $=1.6$, mass density $=2.25$ $\mathrm{g} / \mathrm{cm}^{3}$, borehole diameter $=0.4 \mathrm{~m}$ (1.3 ft.), and source-receiver offset $=3.35 \mathrm{~m}$ (11 ft.). The source function is a Ricker wavelet with a center frequency of $10 \mathrm{kHz}$. There is no attenuation. In this special case, $\delta .=0.231 \mathrm{~ms}$ is greater than $\tau=0.2 \mathrm{~ms}$, so that the wavelets are all separated before other arrivals. 


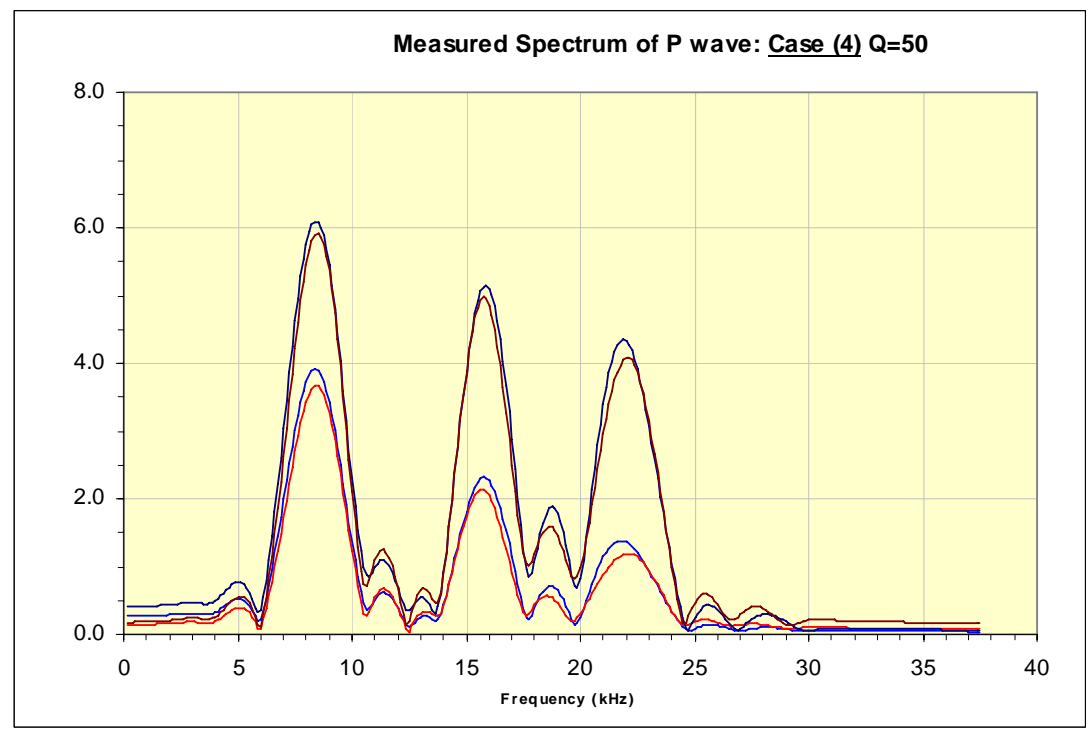

(a)

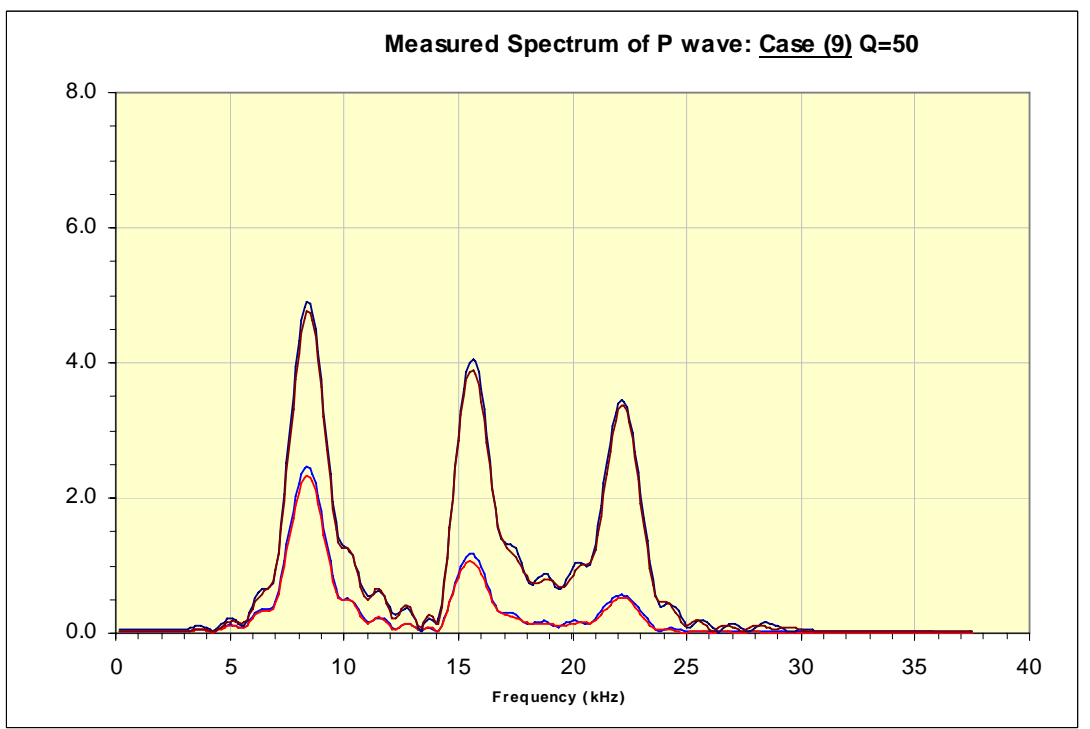

(b)

Figure 3. Amplitude spectra of synthetic (although labeled as "measured") head P waves. The center frequency of the source is $10 \mathrm{kHz}$. Other input parameters are given in Table 1. The upper two curves are $S_{1}$ and $S_{2}$, where no attenuation is present. The dark blue and brown lines denote receivers 1 and 2, respectively. The lower two curves are $M_{1}$ and $M_{2}$, where $Q_{P}$ and $Q_{S}$ are given in Table 1. The blue and red lines denote receivers 1 and 2 , respectively. 
Table 1. Input Parameters for the Synthetic Borehole Head $P$ Waves Used in Figure 3(a) and (b)

\begin{tabular}{|c|c|c|c|c|c|c|c|c|c|}
\hline & $\begin{array}{c}\text { Borehole } \\
\text { radius } \\
(\mathrm{m})\end{array}$ & \multicolumn{2}{|c|}{$\begin{array}{c}\text { Wave } \\
\text { speeds } \\
(\mathrm{m} / \mathrm{ms})\end{array}$} & $\begin{array}{c}\text { Mass } \\
\text { density } \\
\left(\mathrm{gm} / \mathrm{cm}^{3}\right.\end{array}$ & \multicolumn{2}{|c|}{$\begin{array}{l}\text { Quality } \\
\text { factors }\end{array}$} & \multicolumn{2}{c|}{$\begin{array}{c}\text { Source- } \\
\text { receiver } \\
\text { offset } \\
(\mathrm{m})\end{array}$} & $\begin{array}{c}\text { Receiver } \\
\text { spacing } \\
(\mathrm{m})\end{array}$ \\
\hline & $a$ & $V_{P}$ & $V_{S}$ & $\rho$ & $Q_{P}$ & $Q_{S}$ & $1^{\text {st }}$ & $2^{\text {nd }}$ & $\left(z_{1}-z_{2}\right)$ \\
\hline Fig. 3(a) & 0.13 & 2.8 & 1.7 & 2.55 & 50 & 30 & 3.35 & 3.50 & 0.15 \\
\hline Fig. 3(b) & 0.13 & 2.8 & 1.7 & 2.55 & 50 & 30 & 4.88 & 5.03 & 0.15 \\
\hline
\end{tabular}

However, when the external medium is horizontally layered, internal reflections in the borehole are coupled with the reflections and transmissions in the layers. The amplitude spectra of $M_{1}, M_{2}, S_{1}$ and $S_{2}$ become very complicated. In addition, there is no consistency between the amplitude spectra of $M_{1}$ and $M_{2}$ (similarly between $S_{1}$ and $S_{2}$ ). We used finite difference models to synthetically generate the full waveforms of sonic logs in layered media. Figure 4 shows an example of them. It is clear from Figure 4(a) and 4(b) that if we directly use the amplitude spectrum in (a) as $M_{1}$ (or $S_{1}$ ) and that in (b) as $M_{2}$ (or $S_{2}$ ), $M_{1} / M_{2}$ and $S_{1} / S_{2}$ will vary dramatically from frequency to frequency and could often be negative. This mismatch could be improved if we instead use $S_{2} / M_{2}$ and $S_{1} / M_{1}$ as the ratios in (1). But the multiple irregular peaks spanned in a wide frequency range of the amplitude spectra still make the direct use of Equation (1) difficult.

We therefore choose to redefine $M_{1}, M_{2}, S_{1}$ and $S_{2}$ in Equation (1). The objective is to replace them by well defined single wavelets from measured and reference data. We regroup the terms in Equation (2) as follows:

$$
\frac{1}{Q_{P}}=\left(\frac{1}{Q_{0}}-\frac{1}{Q_{1}}-\frac{1}{Q_{3 b}}\right)-\left(\frac{1}{Q_{2}}+\frac{1}{Q_{3 a}}\right)
$$

The first group of terms, $\left(1 / Q_{0}-1 / \tilde{Q_{1}}-1 / Q_{3 b}\right)$ represents the new $M_{1}, M_{2}$, and the second grouped terms, $\left(1 / Q_{2}+1 / Q_{3 a}\right)$ represent $S_{1}$ and $S_{2}$. The expression $\left(1 / Q_{0}-1 / \tilde{Q_{1}}-1 / Q_{3 b}\right)$ implies that in the raw signal, we extract the first borehole wavelet from both internal and external reflections. This step also greatly reduces the complexity of the remaining synthetic correction terms. We can see that $\left(1 / Q_{2}+1 / Q_{3 a}\right)$ merely takes care of the amplitude change of a single wavelet of borehole head wave due to geometrical spreading and transmission loss or gain on its direct path. As a result, the new $M_{1}, M_{2}, S_{1}$ and $S_{2}$ are the amplitude spectra of the first arrived wavelet at both receivers, for both measured data (with unknown $Q_{P}$ ) and reference data (with no attenuation), respectively. For $M_{1}$ and $M_{2}$, the first wavelets are extracted from the measured full waveform sonic. For $S_{1}$ and $S_{2}$, the synthetic waveforms are simply generated for single wavelets. These four single wavelets allow us to apply Equation (1) in a straightforward manner.

We developed two models to support the single wavelet concept in Equation (3). Their details are given in the following sections. 


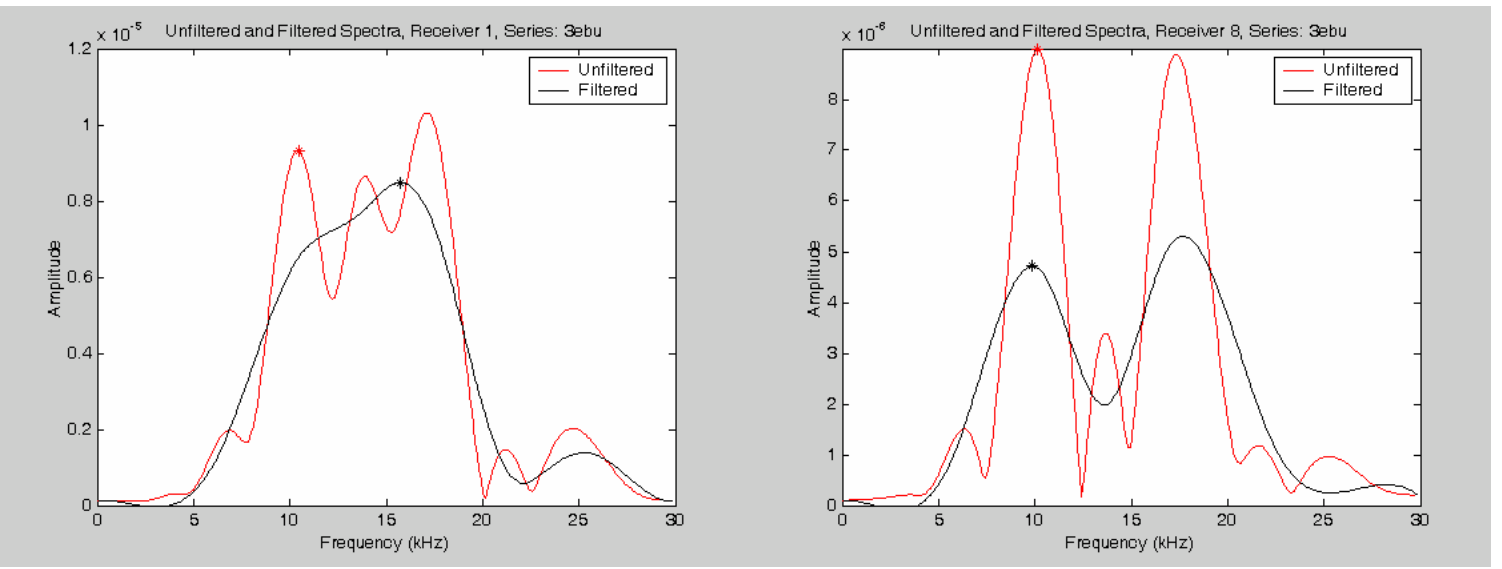

(a)

(b)

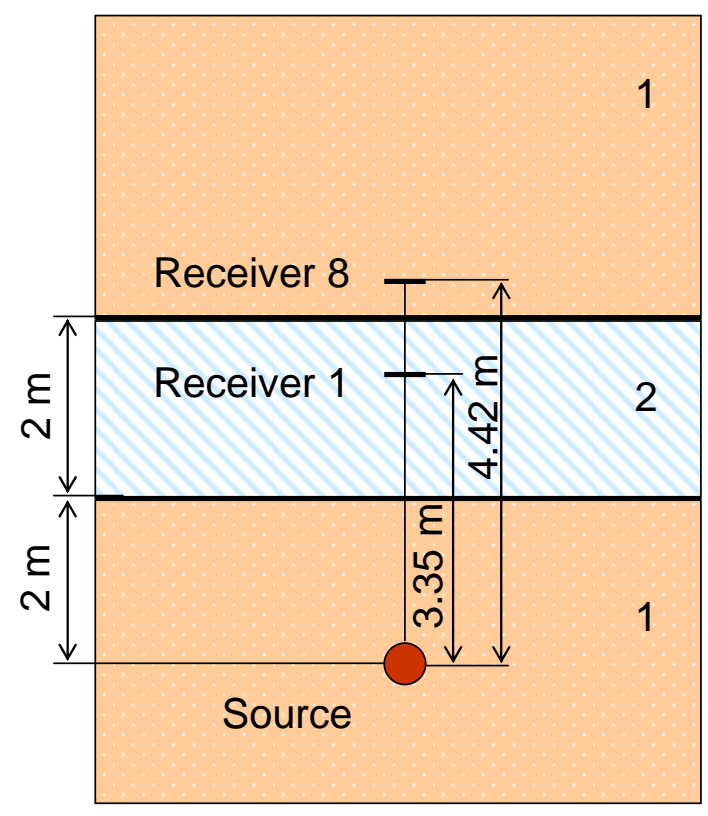

(c)

Figure 4. (a) Amplitude spectrum of borehole head $P$ wave with a center frequency of 10 $\mathrm{kHz}$ at Receiver 1 with offset $=3.35 \mathrm{~m}$ (11 ft.). (b) Same at Receiver 8 with offset $=4.42 \mathrm{~m}$ (14.5 ft.). (c) The geometry and model parameters: P-wave speed $(\mathrm{m} / \mathrm{ms}), S$-wave speed $(\mathrm{m} / \mathrm{ms})$ and mass density $\left(\mathrm{gm} / \mathrm{cm}^{3}\right)$ of materials 1 and 2 are $(4,2,2.7)$ and $(2,1,2.4)$, respectively. In (a) and (b) the red lines are the amplitude spectrum and the black lines are their "smoothed" version. 


\section{B. Theory}

\section{The borehole multiple rays model for measured data}

The first model is a borehole multiple rays model, in which the sonic signal $h(t)$ is assumed to consist of the first borehole ray followed by a number of subsequent rays within a given time gate. The multiple borehole rays consist of the internal reflections within the borehole and reflections from layer interfaces. This model is intended to account for $\left(1 / Q_{0}-1 / \tilde{Q_{1}}-1 / Q_{3 b}\right)$ in Equation (3), and to process measured data $M_{1} / M_{2}$ in Equation (1).

If we let the surrounding medium in the geometry of Figure 1 to be horizontally layered, we can see that $h(t)$ can have the following wavelet representation based on the timing of the rays:

$$
\begin{aligned}
h(t)= & \sum_{n=1}^{N} W_{n}\left[t-t_{P}-\tau / 2-(n-1) \delta\right]+ \\
& \sum_{m=1}^{M} \sum_{n=1}^{N} U_{m n}\left[t-t_{P}-\tau / 2-(n-1) \delta-\xi_{m}\right] \quad t_{P}<t<t_{P}+t_{g}
\end{aligned}
$$

where

$$
\begin{aligned}
W_{n}(t) & =\sum_{k=1}^{5} a_{n k} w_{k}(t) \\
U_{m n}(t) & =\sum_{k=1}^{5} b_{m n k} w_{k}(t)
\end{aligned}
$$

In the above, $n=1,2, \ldots N$ is the label of the borehole rays due to internal reflections, and $W_{n}(t)$ is the $n$th wavelet. Similarly, $m=1,2, \ldots M$ is the label of borehole rays due to reflections from interfaces of the surrounding medium, and $U_{m n}(t)$ is the $n$th wavelet from the $m$ th external reflections. We assume that any wavelet, although having changed its shape on the path, can be expressed as a linear sum of five weighted base functions $w_{k}(t), k=1,2, \ldots 5$. The weighting coefficients of $W_{n}(t)$ are denoted by $a_{n k}$ and those of $U_{m n}(t)$ denoted by $b_{m n k}$ Further, $t_{P}$ is the $P$ wave arrival time; $\tau$ the time duration of the source wavelet; $\delta$ the delay unit between adjacent borehole internal rays; $\xi_{m}$ the additional time delay due to the reflection from formation interfaces, and $t_{g}$ the length of the time gate.

The expressions of the five base functions are given in Appendix A. Among them, $w_{1}(t)$ is the classic Ricker wavelet peaked at $t=0$. The others are its higher order variations. Such a combination allows for shape changes due to factors such as curvature of the borehole, inhomogeneity of the formation, and attenuation. If the source function itself is not a Ricker type, we will define a more suitable set of base functions. Although we call this model a ray model and Equation (4) replies on the timing of rays to interpret and re-construct the waveform, but Equation (4) does not require the wavelength to be much smaller than the borehole caliper. 
In Equation (4), the only unknowns are $a_{n k}$ and $b_{m n k}$. All other parameters are given, or can be determined independently. Among them, $t_{P}$ is picked from the sonic signal; $\tau=2 / f_{c}$ is given; $t_{g}$ is preset; $\xi_{j}$ can be calculated from the distances of interfaces to the receiver and the $P$ wave velocities in the layers. When the surrounding medium is uniform and the borehole diameter is constant, $\delta$ can be determined from the geometry of Figure 1:

$$
\delta=d \sqrt{\frac{1}{V_{f}^{2}}-\frac{1}{V_{P}^{2}}},
$$

where $d$ is the borehole caliper, $V_{f}$ the acoustic wave velocity in the borehole, and $V_{P}$ the $P$ wave velocity in the formation. When $V_{P}$ and $d$ are depth dependent, Equation (5) can be modified in an approximate manner to estimate $\delta$, by assuming $\delta=\left(\delta_{1}+\delta_{2}\right) / 2$.

For $\delta_{1}, V_{P}$ and $d$ are taken from the depth where the ray from the source impinges upon the wall at the critical angle. For $\delta_{2}, V_{P}$ and $d$ are taken from the depth of the entry point as shown in Figure 1. The number of wavelets, $N$, can be determined from the inequality: $(N-1) \delta<t_{g}<N \delta$. Similarly, $M$ can be determined from $t_{g}$ and $\xi_{j}$. Here $t_{g}$ plays an important role in controlling $N$ and $M$.

After the above input parameters are set, a least squares fitting of a measured head wave $h(t)$ in terms of Equation (4) can be carried out to determine $a_{k n}$ and $b_{m n k}$. We then pick the first wavelet, which is the sum of $a_{1 k} w_{\mathrm{k}}(t-\tau / 2)$ for $k=1,2, \ldots 5$. Finally, the amplitude spectra of the first wavelets at the two receivers are used as $M_{1}$ and $M_{2}$ in Equation (1). The accuracy of $a_{k n}$ and $b_{m n k}$ being inverted from the fitting critically depends on the accuracy of $t_{P}$ and $\delta$ in the input.

In the following, we first use Figure 5 as an example to demonstrate the wavelet fitting and inversion of an artificial waveform of three wavelets with periodic time delays. The wavelets and waveform were generated by Equation (4) in the absence of the second term. The coefficients $a_{n k}$ are given in Table 2. Since $t_{P}, \delta$ and $\tau$ were accurately given, the inverted coefficients were found to be exactly the same as the input ones. In Figure 5(a), one cannot see the difference between the input and fitted curves. Figure 5(b) shows the three wavelets extracted from the fitting. Note that the third wavelet is cut by the time gate at $t_{g}=0.3 \mathrm{~ms}$ in the input, but the wavelet fitting was able to recover the entire wavelet.

Next let us look at synthetic borehole sonic full waveforms. Recall that Figure 2 displays the first wavelet (in the green frame) without fitting and extracting. In the synthetic full waveform borehole sonic log in a uniform medium, in Figures 6 and 7, the first wavelets must be extracted through the wavelet fitting. Note that both the "measured" (with attenuation) and "reference" (without attenuation) data were in fact synthetically generated using the wavenumber integral method. Figure 6 shows the fitting for $S_{1}$ and $S_{2}$, which is very good. The extracted first wavelets $M_{1}, M_{2}, S_{1}$ and $S_{2}$ are placed together in Figure 7(a). In Figure 7(b), we see that their amplitude spectra are single wavelets. They maintain the general shape of the source wavelet and have the ideal properties for application of the ASR method used in Equation (1). 


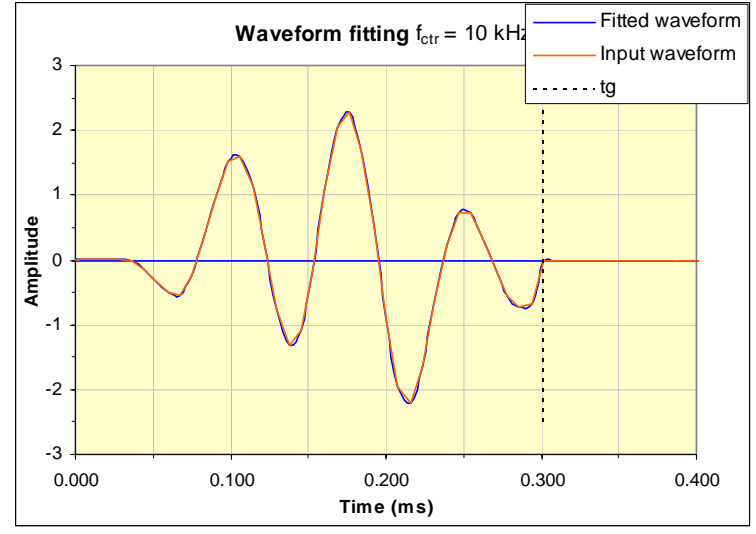

(a)

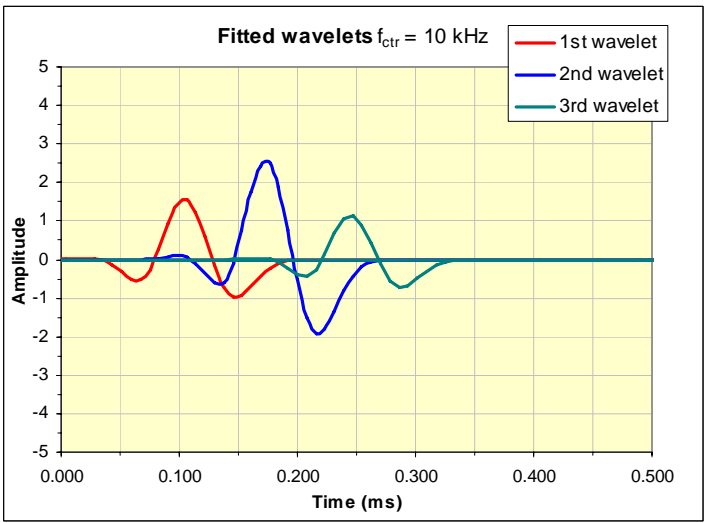

(b)

Figure 5. An example of wavelet fitting of artificial waveform generated by (4). The input parameters are: $t_{P}=0, \tau=0.2 \mathrm{~ms}$, and $\delta=0.7 \mathrm{~ms}$. Input coefficients $a_{n k}$ are given in Table 2. In (a) the orange line is the input waveform and blue line is rebuilt waveforms from the inverted $A_{1}, A_{2}$ and $A_{3}$. In (b) the orange, blue and green lines are the extracted $1^{s t}, 2^{\text {nd }}$, and $3^{\text {rd }}$ wavelets from the fitting, respectively. The time gate is set as $t_{g}=0.3 \mathrm{~ms}$.

Table 2. Input Values of $a_{n k}$ in (4) for Figure 5

\begin{tabular}{|c|c|c|c|c|c|}
\hline$a_{n k}$ & $k=1$ & $k=2$ & $k=3$ & $k=4$ & $k=5$ \\
\hline$n=1$ & 1.00 & 1.00 & 0.50 & 0.25 & 0.50 \\
\hline$n=2$ & 1.50 & 1.20 & 1.00 & 0.50 & 1.00 \\
\hline$n=3$ & 0.80 & 0.90 & 0.40 & 0.20 & 0.30 \\
\hline
\end{tabular}

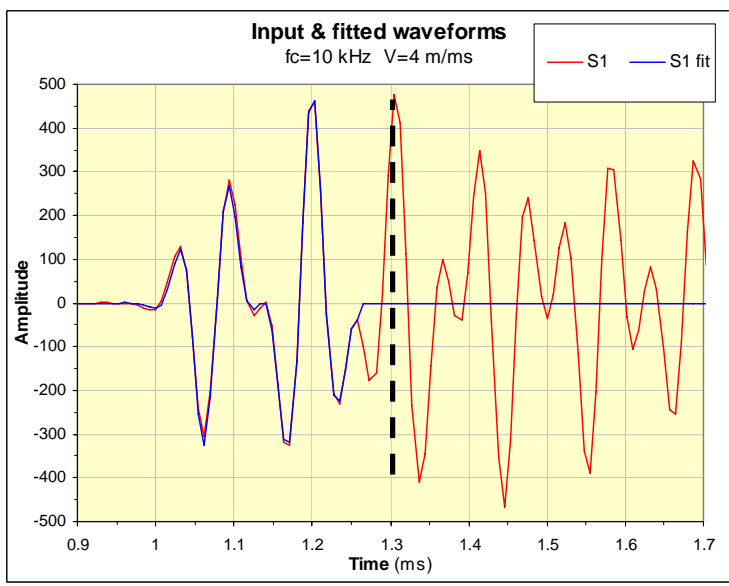

(a)

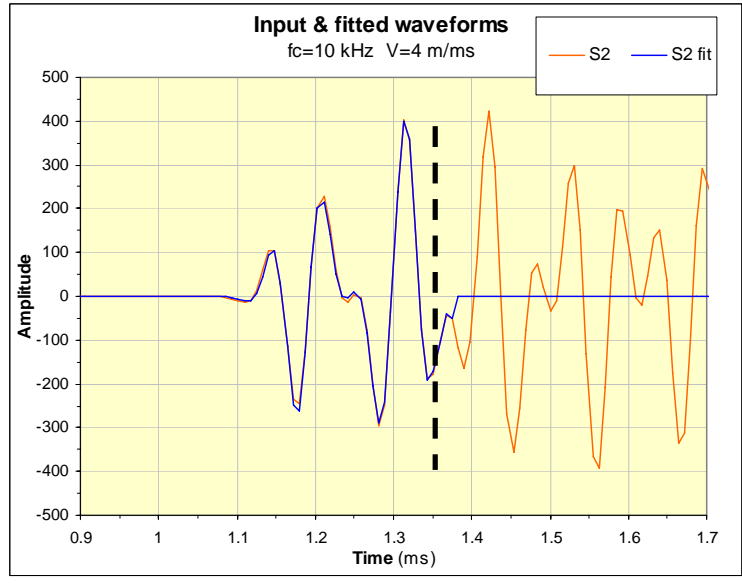

(b)

Figure 6. Input (orange) and fitted (blue) waveforms of synthetically generated reference (no attenuation) borehole sonic data at receivers 1 in (a) and 2 in (b). The dashed line shows the time gate. Model parameters are: $V_{P}=4 \mathrm{~m} / \mathrm{ms}(13 \mathrm{ft} . / \mathrm{ms}), V_{S}=2 \mathrm{~m} / \mathrm{ms}(6.6$ $f t . / \mathrm{ms}), \delta=2.7 \mathrm{gm} / \mathrm{cm}^{3}, d=0.1 \mathrm{~m}\left(0.3 \mathrm{ft}\right.$.), and $f_{c}=10 \mathrm{kHz}$. The source receiver offsets are $3.25 \mathrm{~m}$ (11 ft.) and $3.8 \mathrm{~m}$ (12.5 ft.), respectively. 


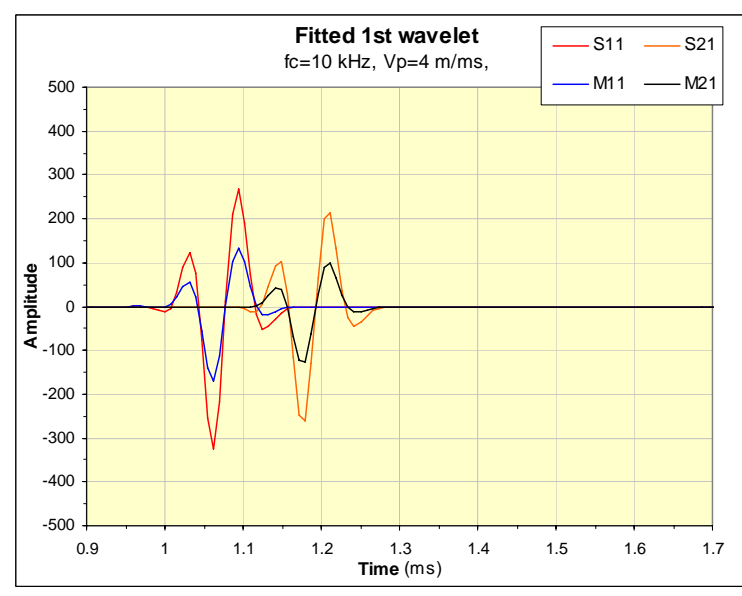

(a)

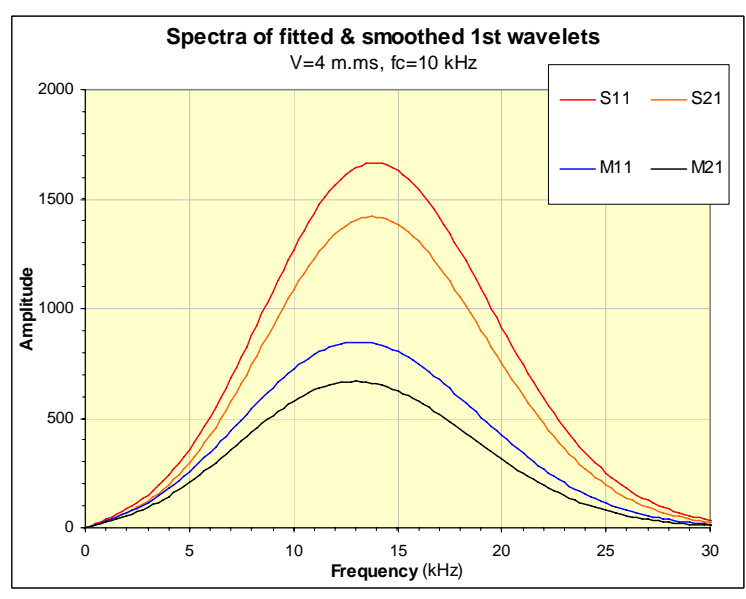

(b)

Figure 7. Following Figure 6, (a) shows the extracted first wavelets of "measured" data $\left(Q_{P}=40, Q_{S}=20\right)$ at receivers 1 and 2 (M11-blue; and M21-black) and reference data (no attenuation) at receivers 1 and 2 (S11-red, S21-orange); and (b) shows their amplitude spectra.

\section{The borehole single ray model for reference data}

The second model is a borehole single ray model in layered formations. This ray on one hand has the transmission coefficients across layer interfaces of the formation similar to a plane wave, and on the other hand undergoes geometrical spreading layer by layer on its wave path. This model is intended to account for $\left(1 / Q_{2}+1 / Q_{3 a}\right)$ in Equation (2), and to generate reference data $S_{1} / S_{2}$ in Equation (1).

The geometrical spreading in an unbounded medium is assumed to obey the following law (Quan et al., 1994):

$$
\frac{A\left(z_{2}\right)}{A\left(z_{1}\right)}=\left(\frac{z_{1}}{z_{2}}\right)^{\gamma}
$$

where $z_{1}$ and $z_{2}$ are the depths of receiver 1 and 2, respectively, $A\left(z_{1}\right)$ and $A\left(z_{1}\right)$ their wave amplitudes, and $\gamma$ is a dimensionless index that depends on the formation properties as well as the borehole caliper.

In the borehole single ray model, we consider the first arriving wavelet that starts from the source in the borehole, propagates as head wave in the surrounding layered formation along the borehole boundary, then re-enters the borehole, and reaches the receiver. Reflections from any interfaces are not considered. Suppose there are $N$ layers covering the depth from the source to the receiver. Let the source depth be 0 . We use subscripts $l, n=1,2 \ldots N$ to denote the labels of the layers such that the $n$th layer is bounded by the depths of $z_{n-1}$ and $z_{n} \ldots$., and that $V_{l}$ is the $P$ wave speed in the $l$ th layer. 
To proceed, we first use the method presented in Appendix B to obtain the geometrical spreading parameter $\gamma_{n}, n=1,2 \ldots N$, in each layer as if it is uniform and unbounded. We assume that waves propagating in a layered medium undergo sectional and continuous geometrical spreading. We accordingly modify the boundary condition to account for the geometrical spreading contrast across an interface. As a result, the transmission coefficient at the $n$th interface, $T_{n}, n=1,2, \ldots N$, becomes frequency dependent and complex valued, involving $z_{n}, \gamma_{n}$ and $\gamma_{n+1}$. Finally, we need the transmission coefficients for the critical refraction at the borehole entry points for the source and receiver indicated in Figure 1. We denote them by $C_{1}$ and $C_{N}$, respectively. We can see from the above that $\gamma_{n}, T_{n}, C_{1}$ and $C_{N}$ are all dispersive, which is consistent with the statements of Paillet and Cheng (1991) and Quan et al. (1994), i.e. the geometrical spreading of the borehole head wave is highly frequency dependent.

From the above, we arrive at the expression of the pressure of the first transmitted wavelet at the receiver depth $z$ :

$p_{T}(z)=p_{0} C_{1} C_{N} \frac{z_{1}^{\gamma_{1}}}{z^{\gamma_{N}}} \prod_{n=1}^{N-1} T_{n} \exp \left(i \omega \frac{z-z_{N-1}}{V_{N}}\right) \exp \left[i \omega \sum_{l=1}^{N-2}\left(\frac{z_{l+1}-z_{l}}{V_{l+1}}\right)\right]$

where $\mathrm{z}_{N-1}<z<z_{N} ; \quad p_{0}$ the pressure at the source, and $\omega$ the circular frequency. The derivation of Equation (7) and $T_{n}$, are given in Appendix C. In the calculation of $C_{1}$ and $C_{N}$, we ignore the curvature of the borehole and replace them by those for a flat interface.

From Equation (7), we can write $S_{1} / S_{2}$ in Equation (1) as

$$
\begin{aligned}
\frac{S_{1}}{S_{2}} & =\frac{p_{T}\left(z_{A}\right)}{p_{T}\left(z_{B}\right)} \\
& =\frac{C_{N_{1}}}{C_{N_{2}}} \frac{z_{B}^{\gamma_{N_{2}}}}{z_{A}^{\gamma_{N_{1}}}} \prod_{n=N_{1}-1}^{N_{2}-1} T_{n}^{-1} \exp \left(i \omega \frac{z_{1}-z_{N_{1}-1}}{V_{N_{1}}}-i \omega \frac{z_{2}-z_{N_{21}-1}}{V_{N_{2}}}\right) \exp \left[-i \omega \sum_{l=N_{1}-2}^{N_{2}-2}\left(\frac{z_{l+1}-z_{l}}{V_{l+1}}\right)\right]
\end{aligned}
$$

where $z_{A}$ and $z_{B}$ are the depths of receivers 1 and 2, respectively. Note that the wave path from the source to the first receiver does not contribute to Equation (8).

Equations (7) and (8) are approximate and have limitations. However, this model should be able to capture the major characteristics of the first wavelet traveling through a layered medium, just like the conventional ray model being used in low frequency seismic problems. Note in our case, the wave path is perpendicular to the interfaces and the energy is concentrated near the borehole wall. This special condition reduces the discrepancy caused by the ray assumption. For example, consider a line force in a layered medium. The line is parallel to the interfaces and the direction of the force is perpendicular to them. It can be shown that the wavefield along the direction of the force is proportional to that caused by a plane wave perpendicular to the interfaces. 


\section{Synthetic Verification}

As discussed in previous sections, the above two models are approximate. We made several efforts to test the accuracy and limits of the models.

We first conducted verification tests on synthetic full waveform borehole sonic logs in uniform formations generated by the classic wavenumber integral method. The agreement was very good. The relative error of $Q$ was found to be within $5 \%$.

The second set of tests was carried out on synthetic full waveform borehole sonic $\operatorname{logs}$ in uniform and layered formations generated by Finite Difference models. The details of the error rate for different cases are given below. The agreement between the input and inverted $Q$ s varies. In general, the agreement is very good when both receivers are in the same layer or half space and away from interfaces. The agreement is poor when the two receivers are on opposite sides of an interface. The wave velocity plays a role. The agreement is better for layers with a higher $P$ wave velocity. In summary, the accuracy needs to be improved.

We are currently working on a rigorous and efficient full waveform solution for a borehole with a varying caliper in horizontally layered viscoelastic media. The approach is based on the Boundary Integral Equation (BIE) method. Upon completion of the BIE full waveform solution, we will use it to test the $Q$ processing algorithm, evaluate the two ray models, identify the limits of the approximations, and improve the accuracy of the algorithm.

We present the result of our $Q$ inversion using 15 receiver pairs from waveforms associated with 17 different earth structures, most of which are layered media. These waveforms were synthetically generated by the Finite Difference. Figure 8 provides the geometries of 17 different earth structures, which are labelled as 1, 2a, 2b, 2c, 2d, 3a, 3b, $3 \mathrm{c}, 3 \mathrm{~d}, 4 \mathrm{a}, 4 \mathrm{~b}, 4 \mathrm{c}, 4 \mathrm{~d}, 5 \mathrm{a}, 5 \mathrm{~b}, 5 \mathrm{c}$ and $5 \mathrm{~d}$. In the cells of the geometry, the number besides the vertical axis is the coordinate of the interface in meters. The ratings of the inverted $Q$ for these geometries are given in the cell below the geometry label. Figure 9 shows the input and inverted $Q$ for all tested cases.

The rock properties of the 17 earth structures are given in Table 3 . The 8 sourcereceiver offsets are given in Table 4, and the 15 receiver pairs are defined in Table 5.

Table 3. P-, S-wave Speeds $(\mathrm{km} / \mathrm{s})$ and Mass Density $\left(\mathrm{gm} / \mathrm{cm}^{3}\right)$ in Earth Structures

\begin{tabular}{|c|c|c|c|c|c|c|c|c|}
\hline $\begin{array}{c}\text { Geometry } \\
\text { label }\end{array}$ & 1 & $2 a$ & 2-bcd & 3-abcd & $4 a$ & 4-bcd & $5 a$ & 5 -bcd \\
\hline $\begin{array}{c}\text { Upper } \\
\text { half } \\
\text { space }\end{array}$ & \multirow{4}{*}{$\begin{array}{l}4, \\
2, \\
2.7\end{array}$} & \multirow{4}{*}{$\begin{array}{l}2, \\
1, \\
2.4\end{array}$} & \multirow[t]{2}{*}{$2,1,2.4$} & $4,2,2.7$ & \multirow[t]{3}{*}{3.2 , } & \multirow[t]{2}{*}{$2,1,2.4$} & \multirow{3}{*}{$\begin{array}{l}2.4, \\
1.2,\end{array}$} & \multirow{2}{*}{$\begin{array}{l}4, \\
2, \\
2.7\end{array}$} \\
\hline Middle & & & & \multirow[b]{2}{*}{$2,1,2.4$} & & & & \\
\hline $\begin{array}{l}\text { layer } \\
\text { (if any) }\end{array}$ & & & & & & \multirow[b]{2}{*}{$3.2,1.6,2.4$} & & \multirow{2}{*}{$\begin{array}{l}2.4, \\
1.2, \\
2.7\end{array}$} \\
\hline $\begin{array}{l}\text { Lower } \\
\text { half } \\
\text { space }\end{array}$ & & & $4,2,2.7$ & $4,2,2.7$ & 2.4 & & 2.7 & \\
\hline
\end{tabular}


Table 4. Source-receiver Offsets

\begin{tabular}{|c|c|c|c|c|c|c|c|c|}
\hline Receiver & 1 & 2 & 3 & 4 & 5 & 6 & 7 & 8 \\
\hline $\begin{array}{c}\text { Offset } \\
\text { (ft) }\end{array}$ & 11 & 11.5 & 12 & 12.5 & 13 & 13.5 & 14 & 14.5 \\
\hline
\end{tabular}

Table 5. Receiver Pairs

\begin{tabular}{|c|c|c|c|c|c|c|c|c|c|c|c|c|c|c|c|}
\hline Label & 1 & 2 & 3 & 4 & 5 & 6 & 7 & 8 & 9 & 10 & 11 & 12 & 13 & 14 & 15 \\
\hline $1^{\text {st }}$ & 1 & 1 & 1 & 1 & 1 & 2 & 2 & 2 & 2 & 3 & 3 & 3 & 4 & 4 & 5 \\
\hline $2^{\text {nd }}$ & 4 & 5 & 6 & 7 & 8 & 5 & 6 & 7 & 8 & 6 & 7 & 8 & 7 & 8 & 8 \\
\hline $\begin{array}{c}\text { Offset } \\
(\mathrm{ft})\end{array}$ & \begin{tabular}{c}
1. \\
\hline
\end{tabular} & 2 & 2.5 & 3 & 3.5 & 1.5 & 2 & 2.5 & 3 & 1.5 & 2 & 2.5 & 1.5 & 2 & 1.5 \\
\hline
\end{tabular}

We plot the results of $Q$ for 15 receiver pairs in the 17 earth models shown in Figure 8 . The total number of cases is 255 , and the agreement for most cases is excellent or good. 


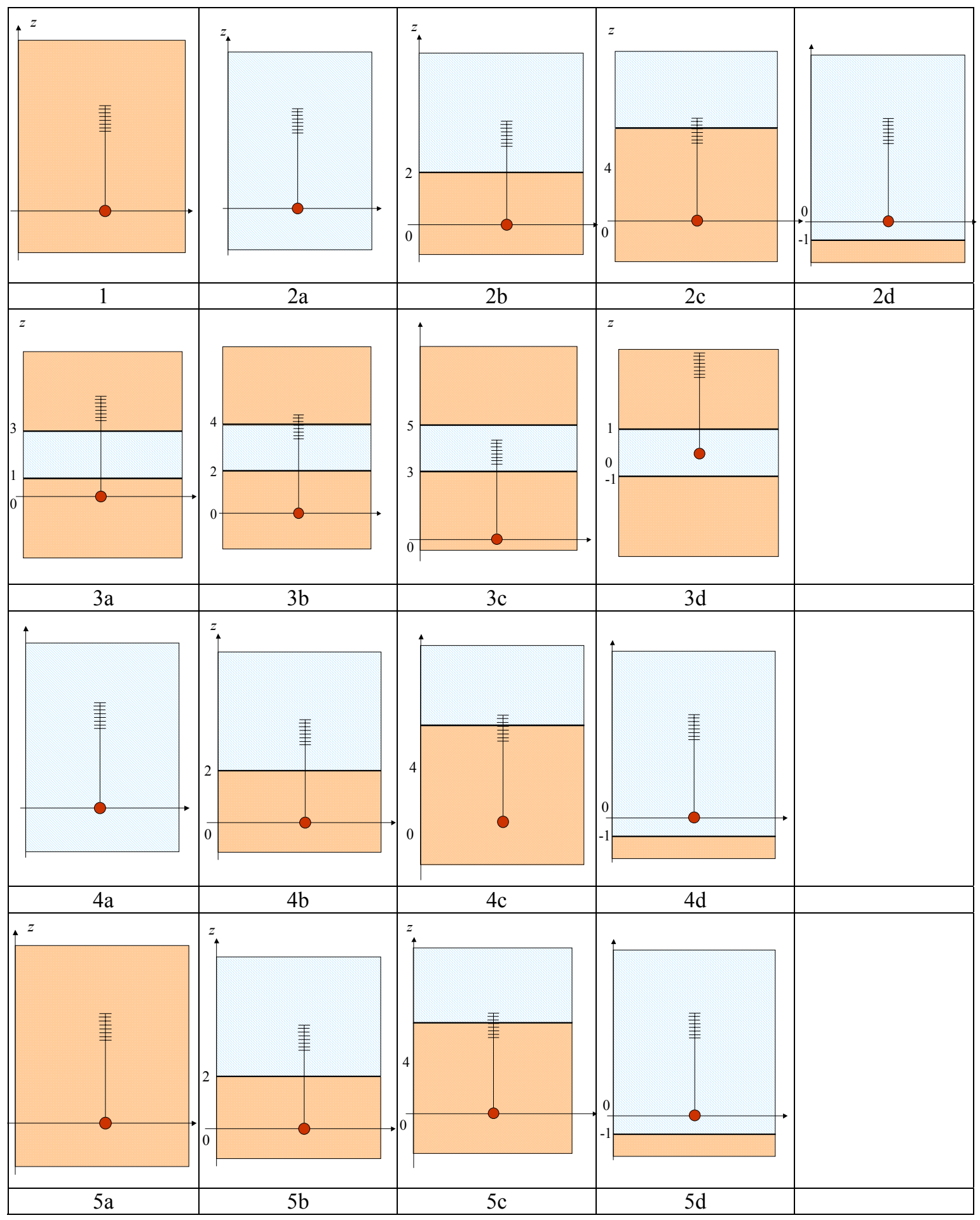

Figure 8. Geometries of the 17 different earth structures for the FD-generated waveforms. The model parameters are given in Tables 3, 4 and 5. 


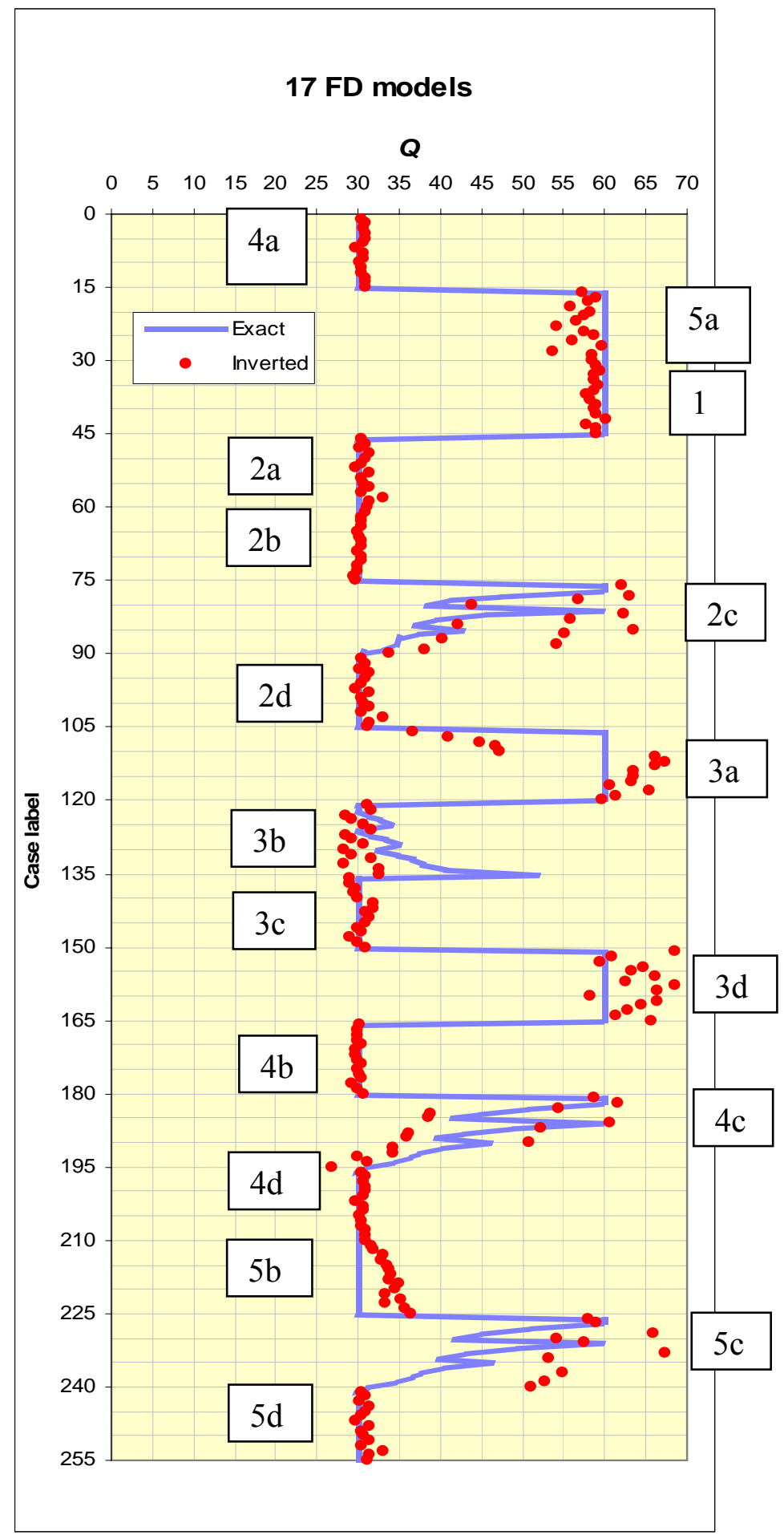

Figure 9. Input (blue line) and inverted (red dots) Qfor 17 different earth models (see Figure 8). The labels of the models are next to the curves. Each model has 15 receiver pairs (see Table 5), each giving an inverted $Q$. The total number of inversion cases is $17 \times 15=255$. 


\section{Use of the Processing Algorithm}

In the $Q$ processing, we require that the borehole sonic data be acquired with controlled gains in a multi-offset source-receiver configuration. The receivers spacing range from half a foot to several feet, which allow us to achieve low, medium or high spatial sampling.

At each source depth, the processing algorithm reads the sonic log, caliper log, and velocity $\log$ from the source depth to the receivers' depths. The sonic log is processed by Equation (4) to extract the first wavelets for a selected pair of receivers. Their amplitude spectra are $M_{1}$ and $M_{2}$ in Equation (1). Simultaneously, we use Equation (8) to synthetically generate the reference single wavelets at the same receivers. Their amplitude spectra are $S_{1}$ and $S_{2}$ in Equation (1). From our numerical tests we found that taking a small area in the neighbourhood of the peak frequency of their amplitude spectra is more reliable than taking the single frequency at the peak. Then we apply Equation (1) to the frequencies within the peak area in an average sense (see Appendix D). The parameters to control the area width and the methods of averaging have been optimally determined through calibration tests on synthetic borehole sonic logs.

Equations (4) and (8) work well if the assumptions and conditions are met. Unfortunately, this is not always the case. Given the complexity of real sonic logs and formation properties, the algorithm must be able to handle some import practical issues when processing the measured data and generating the reference data.

\section{Measured data}

Measured data are much more complex and noisier than synthetic data. However, we found that most of them can be interpreted by the wavelet concept. The key is to be able to determine the timing of the first a couple of events from the patterns of the waveform. Five timing parameters play significant roles in the waveform fitting and wavelet extraction using Equation (4). They are: time gate length $t_{g}, P$ wave arrival time $t_{P}$, borehole ray delay unit $\delta$, interfacial phase delay $\xi_{m}$, and wavelet duration $\tau$.

Clearly, $t_{g}$ directly controls the number of wavelets to be fitted and inverted. If the number of wavelets is either too large or too small, the fitting and inversion will be difficult and unreliable. In addition, a smaller $t_{g}$ can reduce the influence of the dispersive leaky modes on the gated part. From our numerical tests on synthetic data and experience with real data, we tend to make $t_{g}$ small so that $N$ is between 3 and 4 , and $M$ is between 0 and 1 . Thus if one or two external reflections do exist in the second sum, it should come from an interface ahead of the receiver position or an interface behind the source, which makes the determination of $\xi_{m}$ easier. But in any case, $t_{g}$ must not be smaller than $1.5 \tau$. In case the last one, two or even three wavelets are partially cut off by the time gate, the accuracy of the fitting will not be affected. Figure 5 has provided such an example.

Conventional methods for determining the $P$ wave arrival time $t_{P}$ consist of picking the first peak, first trough, and the first zero crossing. We choose to pick the first peak. The first peak has been slightly shifted because of the invasion of the second wavelet. Following Paillet and Cheng (1991), we use cross-correlation in the receiver array to improve the accuracy of $t_{P}$. 
Recall that Equation (4) can be used to calculate the borehole ray delay unit $\delta$ from $d, V_{P}$ and $V_{f}$ provided they are all depth independent. Based on the same concept and similar geometry, we can extend this expression to the layered media as $\delta=$ $\left(\delta_{1}+\delta_{2}\right) / 2$, where both $\delta_{1}$ and $\delta_{2}$ are given by (4), with the values of $d$ and $V_{P}$ being taken at the entry points of the source and receiver (Figure 1), respectively. However, the accuracy of the available $V_{P}$ and caliper logs may not always be reliable. We therefore implemented a wavelet matching algorithm to pick $\delta$ from two adjacent major peaks or troughs of the measured signal. This part of the signal is considered to be a result of two overlapped wavelets with an unknown $\delta$. In this algorithm, we construct a reference signal, which consists of two source wavelets apart by an adjustable $\delta$. We adjust $\delta$ in the reference signal until the best cross correlation between the measured and reference signals are reached. We are aware that the true wavelets in the measured signal are different from the source wavelet both in amplitude and shape, thus this algorithm is still approximate. However, its result should closely represent the $\delta$ needed for Equation (1).

Similarly, the reflection time from a nearby layer interface, $\xi_{m}$, can be estimated from the given $V_{P} \log$, caliper $\log$, including the nearest locations of layer interfaces. Again, they may not be accurate. Using the above wavelet matching algorithm, we can approximately but closely determine $\xi_{m}$ from the measured signal.

\section{Reference data}

The $Q$ processing algorithm does not give accurate results when two receivers are on opposite sides of a high contrast interface. The source of the inaccuracy is in Equation (8) and is due to its approximate nature. Through numerical tests on FD generated borehole sonic data for layered media, we found that the following empirical modifications moderately improve the accuracy of Equation (8) in the above case.

First, $\gamma_{n}$ in Equation (8) is originally defined as the geometrical spreading parameter in the $n$th layer. However, when the $n$th layer is a slow layer where the $\mathrm{S}$ wave velocity is smaller then the acoustic wave velocity in the borehole fluid, then $\gamma_{n}$ should be the average of the geometrical spreading parameters of all layers from the source to the receiver.

Second, we found that replacing $T_{n}$ in (8) by $T_{n}\left[\tilde{1}-\left(\gamma_{n}^{\tilde{n}}-\gamma_{n-1}\right) /\left(\gamma_{n}+\gamma_{n-1}\right)\right]$ yields better results. Note that when the geometrical spreading properties do not change across the $n$th interface the additional factor in the brackets is reduced to 1 . 


\section{Advantages of the algorithm}

Unlike other $Q$ processing techniques, our algorithm is able to physically identify and remove the non-intrinsic factors from the signal and obtain the intrinsic attenuation. The concept is presented in Equation (3). Upon removing all reflected signals, internally and externally, from the sonic log, the only correction needed is a reference synthetic solution of the first arriving wavelet for a borehole in a layered medium. The method for achieving the goal of Equation (3) is given in Equation (4) for the sonic log and in Equation (8) for the reference solution.

The second advantage of this algorithm is that the single wavelet concept of the processing provides an ideal condition for the use of the ASR method. Figure 5 demonstrates that if the input timing parameters are accurate, the unknown coefficients of the base functions, and hence the wavelets, can be inverted accurately. In Figures 6 and 7, we showed that the extracted first wavelets at any receivers have a modified shape of the source wavelet. Their amplitude spectra are well shaped and single peaked, which are ideal to apply Equation (1) as well.

\section{Approximations}

There are two source of inaccuracy in the algorithm. One is in Equation (8) for the reference data. Equation (8) is based on a ray approximation of the head wave and an assumption regarding the geometrical spreading relation across an interface.

The other source of inaccuracy is in the use of Equation (4). Although this equation does not involve approximation, the accuracy of the input timing parameters such as $t_{P}, \delta, \xi_{m}$ and $\tau$. affect the accuracy of the result to various extent.

\section{Limitations}

This algorithm has been developed to account for depth dependent variations of both borehole caliper and formation inhomogeneity.

Paillet and Cheng (1991) pointed out that borehole caliper size significantly affects the properties of the borehole head wave. In our both ray models, the borehole caliper $(d)$ does, implicitly, play an important role. The algorithm reads the caliper log at every depth as input. In the borehole multiple rays model for measured data (Equation (1)), the delay unit $\delta$ controls the timings of all wavelets. As seen in Equation (6), $\delta$ is proportional to $d$. In the borehole single ray model for reference data (Equation (8)), the geometrical spreading parameters $\gamma$ in the layers are needed to obtain the modified transmission and reflection coefficients. We will show in Appendix B that $\gamma$ is strongly dependent on $d$, among other parameters. Numerical tests showed that the algorithm handles moderate caliper changes properly, but has difficulty when the change is abrupt.

Similarly, the $V_{P} \log$ is the major input to Equation (8). We also found that the algorithm handles moderate $V_{P}$ well, but still has difficulty when the change is abrupt.

We are aware of all the limitations of our algorithm and we are making efforts to improve the ray models. For the reference data, we are working on a rigorous full waveform solution for a borehole with depth dependent caliper in a horizontally layered 
medium. In addition, we are carrying out ultrasonic simulation of the same model. For the measured data, we will further improve our ability to pick $t_{P}, \delta$, and $\xi_{m}$ accurately.

\section{Formation anomalies}

The formation and borehole may have anomalies that are beyond the scope of the model of a fluid-filled borehole in a layered linearly elastic solid. These anomalies include 3D inhomogeneity, nonlinearity, etc. They are beyond our scope. In such cases, the algorithm will fail, but at the same time will provide some helpful information about the anomaly.

\section{Observations based on practical applications}

We have applied the processing algorithm to extract $Q$ logs from two data sets. One from a sonic log acquired in a south Florida aquifer (Parra et al., 2004), and the second from a sonic log from an oil reservoir in northeast Texas (Parra et al., 2006). In the Florida aquifer we evaluated the attenuation log using porosity logs, rock physical properties, and a $Q$ model based on porelasticity. We also use vuggy and matrix porosity logs obtained by joint inversion of resistivity and velocity logs by Kazatchenko et al. (2004). We integrated the porosity logs with the lithology to visualize a region that contains different facies that give different acoustic signatures. The consistence of the integration supports the attenuation log data associated with the different lithological units. In the second site, the oil reservoir is a sand-shale sequence, which attenuation log correlates with the lithology, including a sandstone zone partially saturated with oil and water. The $Q \log$ detected a zone that contains oil and water, which has suggested fluid motion between the oil and water in the presence of a gas phase. This has reduced the acoustic energy. In addition, with the help of the petrophysics, the $Q \log$ has allowed us to evaluate the acoustic attributes in the micro-seismogram. In particular, we have observed an increased in attenuation in a few isolated short intervals when low velocity shale is bounded by a high velocity limestone. This has been useful for explaining the reservoir petrophysics and the conditions of the geological markers. In this case the attenuation between shale and limestone (both geological units have near zero permeability) occurs where not fluids are present. Alternatively, the velocity contrasts between the shale and sands (where fluids are present) the algorithm has provided $Q$ values that correlate with the partially saturated sands 


\section{E. Conclusions}

The attenuation algorithm automatically corrects the sonic data for scattering, geometrical spreading and borehole effects. The algorithm was tested and verified with synthetic data. The synthetic data generated with the solution of a point source in fluidfilled borehole gave an error within five percent; while the synthetic data generated using the Finite Difference method gave various degree of accuracy. The result depends on the position of the receivers relative to the layer interface. We observed that, when the receivers cross a velocity boundary, the algorithm is not stable, but when both receivers are on either side of the boundary, the results are stable. To handle instability problems when receivers cross a velocity boundary, we are developing a boundary value problem for a point source in a fluid-filled borehole surrounded by multilayered earth. This solution will allow us to correct $Q$ logs for coupling between the borehole and the layers for any position of the receivers at velocity boundaries. Nevertheless, the applicability of the preset algorithm to real sonic data (Parra et al. 2004 and 2006) has demonstrated its usefulness to characterize the pore structure in a carbonate aquifer, and to detect fluids in an oil reservoir. 


\section{APPENDIX A \\ BASE FUNCTIONS FOR WAVELET CONSTRUCTION}

We assume that a wavelet $W_{n}(t)$ can be written as a linear weighted sum of 5 base functions:

$W_{n}(t)=\sum_{k=1}^{5} a_{k n} w_{k}(t) \quad 0<t<\tau$

where

$w_{1}(t)=\left(1-\frac{8 \pi^{2} t^{2}}{\tau^{2}}\right) \exp \left(-\frac{4 \pi^{2} t^{2}}{\tau^{2}}\right)$

is the classic Ricker's wavelet. In (A-2), $\tau$ is the nominal duration of the wavelet. Other base functions are its higher order variations:

$$
\begin{aligned}
& w_{2}(t)=\frac{2 \pi t}{\tau} \exp \left(-\frac{4 \pi^{2} t^{2}}{\tau^{2}}\right) \\
& w_{3}(t)=\frac{8 \pi^{3} t^{3}}{\tau^{3}} \exp \left(-\frac{4 \pi^{2} t^{2}}{\tau^{2}}\right) \\
& w_{4}(t)=\left(1-\frac{64 \pi^{4} t^{4}}{3 \tau^{4}}\right) \exp \left(-\frac{4 \pi^{2} t^{2}}{\tau^{2}}\right) \\
& w_{5}(t)=\frac{32 \pi^{5} t^{5}}{\tau^{5}} \exp \left(-\frac{4 \pi^{2} t^{2}}{\tau^{2}}\right)
\end{aligned}
$$




\section{APPENDIX B GEOMETRICAL SPREADING OF BOREHOLE HEAD $P$ WAVE}

(Quan et al., 1994) proposed a model of the geometrical spreading of the head wave for a borehole in an unbounded uniform medium. The mathematical form of this model is (see A3):

$$
\frac{A\left(z_{2}\right)}{A\left(z_{1}\right)}=\left(\frac{z_{1}}{z_{2}}\right)^{\gamma}
$$

Where $z_{1}$ and $z_{2}$ are the source-receiver offsets for receivers 1 and 2, respectively, and $A$ denotes the amplitude. For a borehole with a uniform diameter in a uniform formation, the dimensionless geometrical spreading parameter $\gamma$ can be determined empirically from the synthetic borehole full waveforms. The model parameters needed to uniquely generate the waveforms are: i) frequency $(\omega)$; ii) source-receiver offset $(z)$; iii) $P$ wave speed $\left(V_{P}\right)$; iv) $S$ wave speed $\left(V_{S}\right)$; v) mass density $(\rho)$; and vi) diameter of the borehole $(d)$. For a gated portion of the head wave from this full waveform, an additional parameter is the gate length $t_{g}$. Consequently, the geometrical spreading index $\gamma$ in the above equation should be a unique function of $\left(\omega, V_{P}, V_{S}, \rho, d, t_{g}\right)$ and should fit all values of $z$.

Using numerous combinations of model parameters, we were further able to obtain a well correlated empirical relation between $\gamma$. and $\left(V_{P}, V_{S}, d\right)$ :

$\gamma(\omega)=A(\omega) V_{P}+B(\omega) \frac{V_{P}}{V_{S}}+C(\omega) d+D(\omega)$

The mass density $(\rho)$ was found to have insignificant influence on $\gamma$. As an example, we generated synthetic full waveforms at eight source-receiver offsets using 5321 different sets of model parameters. The circular frequency was $\omega=10 \times 2 \pi \mathrm{kHz}$. The time gate length was $0.3 \mathrm{~ms}$. We first conducted a regression fit of (B-1) for each set of parameters to obtain $\gamma$. Then we used the 5321 sets of $\gamma, V_{P}, V_{S}$ and $d$ to conduct a regression fit of (B-2). We obtained $A=0.6471, B=0.2445, C=0.3902, D=0.3462$ when $V_{S}>1.5 \mathrm{~m} / \mathrm{ms}(5 \mathrm{ft} / \mathrm{ms})$; and $A_{=}=0.0108, B \tilde{=}=0.0792, C=0.5928, D=1.4608$ when $V_{S}<1.5$ $\mathrm{m} / \mathrm{ms}(5 \mathrm{ft} / \mathrm{ms})$. We in turn applied these constants to (B-2) to predict $\gamma$ for these 5321 cases. We found the average relative standard deviation between the predicted $\gamma$ from (B-2) and the original $\gamma$ from (B-1) is $6 \%$.

The relationship revealed by (B-2) implies that for fast formations, $\gamma$ is between 1 and 1.5, and increases with increased $V_{P}, V_{S}$ or $d$. For slow formations, $\gamma$ is between 1.4 and 1.7. It increases with decreased $V_{P}$ or increased $V_{S}$ at a lower rate, and increases with increased $d$ at a higher rate. In all formations, $\gamma$ is sensitive to $d$ and increases with increased $d$. The above results are valid for $\omega=10 \times 2 \pi \mathrm{kHz}$. 


\section{APPENDIX C \\ REFLECTION AND TRANSMISSION OF WAVES \\ WITH GEOMETRICAL SPREADING}

\section{Geometrical spreading in an unbounded medium}

Following Quan et al. (1994), the displacement and pressure of a borehole head wave in a uniform unbounded medium can be expressed as

$u(z)=\frac{A}{z^{\gamma}} \exp \left[\frac{i \omega z}{V}\right]$

where $u$ represents either displacement $(w)$ or pressure $(p), \mathrm{z}$ is the source-receiver offset, $V$ the velocity of the head wave, $\omega$ the circular frequency, and $\gamma$ a dimensionless parameter. Accordingly the ratio of $u$ at two receivers is

$$
\frac{u\left(z_{2}\right)}{u\left(z_{1}\right)}=\left(\frac{z_{1}}{z_{2}}\right)^{\gamma} \exp \left[\frac{i \omega\left(z_{2}-z_{1}\right)}{V}\right]
$$

We assume that (C-2) can be extended to a layered medium when both receivers are within a layer while the source can be in any layer. Then the boundary conditions at the interfaces will give us the sectional geometrical spreading relation across the interfaces.

\section{Reflection and transmission across an interface}

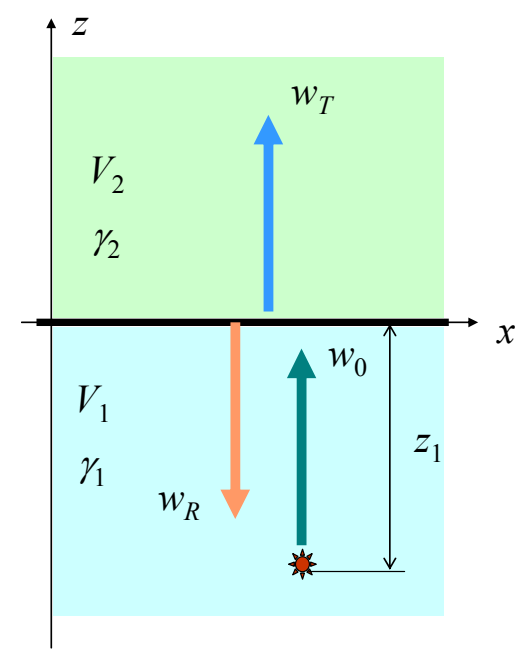

Figure C-1. Wave reflection and transmission at an interface

Following the geometry and notations of Figure $\mathrm{C}-1$, the interfacial condition for the continuity of displacement is written as

$$
\left[w_{0}(z)+w_{R}(z)\right]_{z=0}=\left.w_{T}(z)\right|_{z=0}
$$


where $w_{0}$ is the wave field when the source medium is unbounded, $w_{R}$ the reflected wave, and $w_{T}$ the transmitted wave. Following the above assumption, their expressions are

$$
\begin{array}{ll}
w_{0}(z)=\frac{1}{\left(z_{1}+z\right)^{\gamma_{1}}} \exp \left(\frac{i \omega\left(z_{1}+z\right)}{V_{1}}\right) & 0 \geq z \geq-z_{1} \\
w_{R}(z)=w_{0}(0) R \frac{z_{1}^{\gamma_{1}}}{\left(z_{1}-z\right)^{\gamma_{1}}} \exp \left(\frac{-i \omega z}{V_{1}}\right) & 0 \geq z \geq-z_{1} \\
w_{T}(z)=w_{0}\left(z_{1}\right) T \frac{z_{1}^{\gamma_{1}}}{\left(z_{1}+z\right)^{\gamma_{2}}} \exp \left(\frac{i \omega z}{V_{2}}\right) & 0 \leq z<\infty
\end{array}
$$

Equations (C-3) through (C-6) lead to

$$
(1+R)=T z_{1}^{\gamma_{1}-\gamma_{2}}
$$

where $R$ and $T$ are reflection and transmission coefficients to be determined.

The interfacial condition for the continuity of pressure requires that

$$
V_{1}^{2} \rho_{1}\left(\frac{\partial w_{0}(z)}{\partial z}+\frac{\partial w_{R}(z)}{\partial z}\right)_{z=0}=V_{2}^{2} \rho_{2}\left(\frac{\partial w_{T}(z)}{\partial z}\right)_{z=0}
$$

which can be rewritten as

$$
b I_{1}(1-R)=c I_{2} T
$$

where

$$
I_{1}=V_{1} \rho_{1} \quad I_{2}=V_{2} \rho_{2} \quad b_{1}=1+\frac{i V_{1} \gamma_{1}}{\omega z_{1}} \quad b_{2}=1+\frac{i V_{2} \gamma_{2}}{\omega z_{1}}
$$

and $\quad 1$ and 2 are the mass densities. Upon solving Equations (C-7) and (C-8), we obtain the complex reflection and transmission coefficients below.

$$
R=\frac{I_{1}-c I_{2}}{b I_{1}+c I_{2}} \quad T=z_{1}^{\gamma_{2}-\gamma_{1}} \frac{(1+b) I_{1}}{b I_{1}+c I_{2}}
$$

Not that in the absence of geometrical spreading, we have $\gamma_{1}=\gamma_{2}=0$ and $b=c=1$. Then (C9) is reduced to the well known expressions: $R=\left(I_{1}-\tilde{I} I_{2}\right) /=\left(I_{1}+I_{2}\right), T=2 I_{1} /\left(I_{1}+I_{2}\right)$. 


\section{Reflection and transmission across an interface in layered media}

Following the notations in Figure C-2, we can apply the sectional geometrical spreading relation to all the interfaces between the source and the receiver.

Let $z_{l}, l=1,2, \ldots$ denote the coordinates of the interfaces. Applying (C-9) to the $n$th interface, we have

$$
R_{n}=\frac{I_{n}-c_{n} I_{n+1}}{b_{n} I_{n}+c_{n} I_{n+1}} \quad T_{n}=z_{n}^{\gamma_{n+1}-\gamma_{n}} \frac{\left(1+b_{n}\right) I_{n}}{b_{n} I_{n}+c_{n} I_{n+1}}
$$

where

$$
I_{n}=V_{n} \rho_{n}
$$$$
b_{n}=1+\frac{i V_{n} \gamma_{n}}{\omega z_{n}} \quad c_{n}=1+\frac{i V_{n+1} \gamma_{n+1}}{\omega z_{n}}
$$

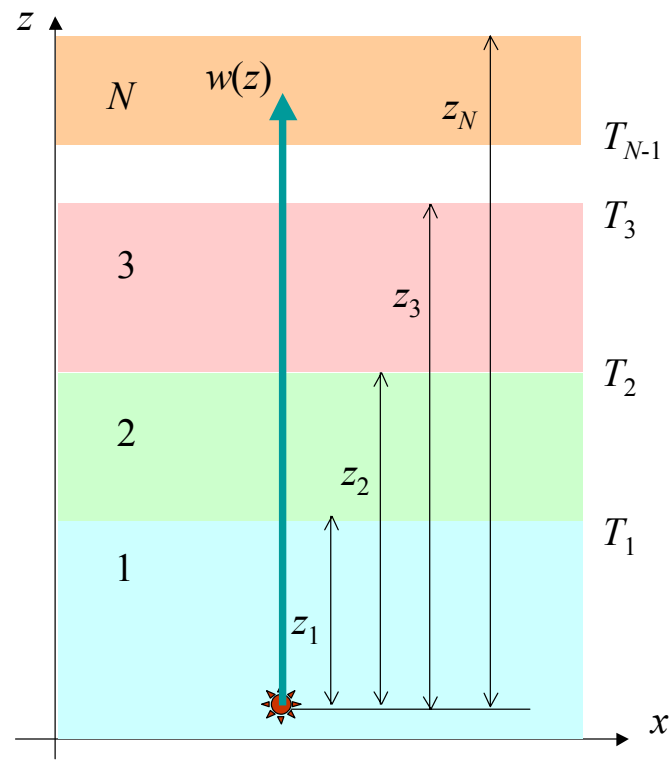

Figure C-2. Geometry of a layered medium 


\section{APPENDIX D \\ APPLICATION OF ASR METHOD TO A SPECTRAL PEAK}

Through numerous numerical tests on synthetic borehole waveforms, we found that simply applying the ASR, i.e. Equation (1), to the peak of the amplitude spectrum often yield an inaccurate result. Therefore we define a peak area for each spectrum to apply the equation in an average sense. There are many options to define the peak area and to take average in the peak area.

We determined that the peak area should cover the frequency range where the amplitudes are above $75 \%$ of the peak amplitude. The table below provides the four averaging schemes that give good results. We choose to take the mean value of them.

\begin{tabular}{|c|c|c|}
\hline \multirow{2}{*}{$\begin{array}{c}\text { Amplitude ratio } \\
\text { options }\end{array}$} & $\begin{array}{c}|c| \\
\text { Average of peak frequencies } \\
\text { of }\left(S_{1}, S_{2}, M_{1}, M_{2}\right)\end{array}$ & $\begin{array}{c}\text { Averages of peak frequencies of } \\
\left(S_{1}, S_{2}\right) \text { and }\left(M_{1}, M_{2}\right)\end{array}$ \\
\hline $\begin{array}{c}\text { Ratio of } \\
\text { mean amplitude }\end{array}$ & $Q_{1}$ in Eq. (F-1) & $Q_{2}$ in Eq. (F-2) \\
\hline $\begin{array}{c}\text { Mean of } \\
\text { amplitude ratios }\end{array}$ & $Q_{3}$ in Eq. (F-3) & $Q_{4}$ in Eq. (F-4) \\
\hline
\end{tabular}

$Q_{1}^{-1}=\frac{8 V_{P}}{\left(\omega_{\text {peak } S 1}+\omega_{\text {peak } S 2}+\omega_{\text {peak } M 1}+\omega_{\text {peakM } 2}\right)\left(z_{2}-z_{1}\right)} \ln \frac{A_{\text {mean } M 1} A_{\text {mean } S 2}}{A_{\text {mean } M 2} A_{\text {mean } 1}}$

where $A_{\text {mean }}$ denotes the mean amplitude of the peak area of the specified spectrum.

$$
Q_{2}^{-1}=\frac{V_{P}}{z_{2}-z_{1}}\left(\frac{4}{\omega_{\text {peakM } 1}+\omega_{\text {peakM2 }}} \ln \frac{A_{\text {mean } M 1}}{A_{\text {mean } M 2}}-\frac{4}{\omega_{\text {peak } S 1}+\omega_{\text {peakS } 2}} \ln \frac{A_{\text {mean } S 1}}{A_{\text {meanS } 2}}\right)
$$

Note that the peak frequency of $S_{1}$ and $S_{2}$, or $M_{1}$ and $M_{2}$, may not coincide, so their peaks areas cover different frequency ranges. In $(\mathrm{D}-3,4)$ below, the mean values are taken from the extended frequency range that covers both $S_{1}$ and $S_{2}$. The same is applied to the peak areas of $M_{1}$ and $M_{2}$. Unlike in (D-1, 2), $A_{S 1}, A_{S 2}, A_{M 1}, A_{M 2}$ vary with frequency in (D-3, 4).

$$
\begin{aligned}
& Q_{3}^{-1}=\frac{V_{P}}{z_{2}-z_{1}} \frac{8}{\omega_{\text {peakM } 1}+\omega_{\text {peak } 2}+\omega_{\text {peak } S 1}+\omega_{\text {peak } S 2}}\left(\operatorname{mean}\left[\ln \frac{A_{M 1}(\omega)}{A_{M 2}(\omega)}\right]-\operatorname{mean}\left[\ln \frac{A_{S 1}(\omega)}{A_{S 2}(\omega)}\right]\right) \\
& Q_{4}^{-1}=\frac{V}{z_{2}-z_{1}}\left(\frac{4}{\omega_{\text {peakM } 1}+\omega_{\text {peakM } 2}} \operatorname{mean}\left[\ln \frac{A_{M 1}(\omega)}{A_{M 2}(\omega)}\right]-\frac{4}{\omega_{\text {peak } S 1}+\omega_{\text {peak } 2}} \text { mean }\left[\ln \frac{A_{S 1}(\omega)}{A_{S 2}(\omega)}\right]\right)
\end{aligned}
$$




\section{WAGGONER RANCH SURFACE GEOLOGY AND PETROPHYSICS}

One of our objectives was to apply the processing techniques developed in this research program to a real oil reservoir. The understanding of the geological site geology and petrophysics are important for proof of concepts and to establish the usefulness Q as well as the applicability of the subsurface and surface algorithms. The first part of this section describes the Waggoner geology, and the second part provides relationships between the different rock physical properties of the reservoir. The core, well log, and seismic data recorded in this site were reported in the second DOE Annual report that was issued in August 2003.

\section{A. Geology}

The Waggoner Ranch comprises 1000 square miles reaching across six counties in north central Texas, but oil and gas production is associated almost exclusively with the Red River Carbonate Platform stretching through the northeast part of the ranch, which spans parts of Wichita and Wilbarger counties. More than 13,000 wells have been drilled on the ranch since the early 1900s.

Hydrocarbon production is primarily from shallower Permian horizons (less than 2,500 feet), where thin sandstone and limestone formations represent alternating, fairly rapid transgressive and regressive marine sequences. The more significant of these formations are the Thomas, Gunsight, Dyson, and Milham sandstones. Productive zones have also been found at depths to about 1585 meters $(5,200$ feet) in Ordovician carbonates, where porosity has been preserved at the tops of intervals. These formations are the Canyon, Strawn, Caddo, and Ellenburger.

Surface geology at the ranch is represented by eight depositional units (from Hentz and Brown, 1987):

1. Seymour Fm. (Pleistocene) - A massive, silty sand, the Seymour comprises a thin bedded unit [0.3-3 m (1-10 ft.) deep], and a thick bedded unit [3-23 m $(10-75 \mathrm{ft}$.$) thick] containing volcanic ash and numerous reworked$ invertebrates.

2. Windblown sheet deposits (Holocene and Pleistocene) cover portions of the Seymour - they are composed of silt, sand, and clay, and are up to $6 \mathrm{~m} \mathrm{(20} \mathrm{ft.)}$ thick.

3. Clear Fork Group (Permian) - This group contains mudstone, siltstone, sandstone, dolomite, limestone, and gypsum members; vertebrate fossils are common in the mudstone. The Clear Fork varies from 366-427 m (1200-1400 ft.) thick.

4. Petrolia Fm. (Permian) - This formation is made up of mudstone, shale, conglomerate, limestone, and several sandstone members. Rare vertebrate footprints have been found in the formation, which is from 110-122 $\mathrm{m}$ (360$400 \mathrm{ft}$.) thick. 
5. Holocene-age alluvium forms floodplain and channel deposits comprising sand, silt, clay, gravel, and some eolian dunes. Alluvium is up to $9 \mathrm{~m} \mathrm{(30} \mathrm{ft.)}$ thick.

6. Lueders Fm. (Permian) - Contains the Lake Kemp and Maybelle limestones and some interbedded shale and dolomite. It is $15-23 \mathrm{~m}(50-75 \mathrm{ft}$.) thick.

7. Waggoner Ranch Fm. (Permian) - Primarily reddish-brown mudstone in badland terrain, this formation also consists of shale, limestone, siltstone, sandstone, and conglomerate. Sandstone units of the Waggoner Ranch are thin and lenticular; limestone and dolomite occur as nodular zones in mudstones and as thin, mappable beds. A variety of plant, vertebrate, and invertebrate fossils are found in the formation, which ranges from 116-128 m (380-420 ft.) thick.

8. Holocene/Pleistocene-age fluviatile terrace deposits, up to $9 \mathrm{~m}$ (30 ft.) thick, made up of gravel, sand, and silt, with granule- to cobble-sized clasts of metamorphic rock, milky quartz, chert, and igneous rock that appears to be from far western sources.

The area of interest in this study is located in northeast Wilbarger County, just west of Lake Electra. This portion of North Texas is renowned for its exposures of Permian age stratigraphy. The project area surface stratigraphy lies in the Waggoner Ranch Formation, which is the uppermost formation of the Wichita Group and early Leonardian in age. The Waggoner Ranch is bound by the Petrolia Formation at its base and by the Leuders, which overlies it and marks the base of the Clearfork Group.

The 1000-acre seismic program (West Electra Lake) was acquired by dynamite in S.P.R.R. Section 1, part of H\&TC Block 14, Section 136, the northwest portion of H\&TC Block 5, Section 6, and the western portion of H\&TC Block 13, Section 1. An additional 356 acres (West Electra Lake Extension) of seismic was acquired by accelerated weight drop on adjoining acreage in H\&TC Block 5, Section 6 of Wilbarger County, Texas, from the western shoreline of Lake Electra to the west and north line of Section 6 . Petrophysical data to be collected at new wells in this area includes general induction logs, lateral sonic logs, sidewall cores, FMI logs, density neutron logs, micrologs, and possibly dip meter runs.

Four new wells were drilled at the West Electra Lake region of the Waggoner Ranch in the 3-D seismic survey areas. Three of the wells found oil in the Upper Milham Sand; the fourth (in a different section and formation) was a dry hole, and was plugged and abandoned. The three producing wells (Waggoner-Cadence West Electra Lake wells $\# 1,2$, and 3) were logged. In addition to being logged extensively, the \#3 well had about 34 meters (112 feet) of core pulled during the drilling process. The data from these three wells is given in the DOE 2004 Annual Report.

In the study area, the Waggoner Ranch Formation is characterized at the surface by three components: gently rolling hills that cover most of the acquired area, a bluff on the southern edge with approximately $15 \mathrm{~m}$ (50 feet) of relief, and the poorly vegetated 
drainage system of Beaver Creek to the west and south. Characterization of the gentle sloping areas came from 6-m (20-foot) shot hole samples from the West Electra Lake acquisition. The samples contained primarily reddish-brown or medium grey shale/mudstone. Quartzite gravel and pebbles were encountered in the low relief drainage areas, while a few sites contained limestone or sandstone. The bluff on the southern edge of the extension shoot has a surface of pebbles and clay soil with reddish to gray sandstone members from 3 to 6 meters (10 to 20 feet) thick directly underlying it. Red and gray mudstone and shale with some calcite and dolomite nodules underlay the sandstone. Beaver Creek and its tributaries have exposed the stratigraphy of the area by widespread erosion, creating a broad drainage basin. This erosion has led to badlands topography, as the creek has cut through the soft shale and mudstone of the paleostratigraphy. In addition, Quaternary unconsolidated silt, sand, and clay have filled much of the bounding tributaries and confining elevations of the Electra Lake reservoir.

\section{B. Petrophysics}

\section{Well log crossplots}

In this section, we examine the well logs of Waggoner well \#3, and crossplot several parameters from the reservoir interval, including $Q$ values extracted from the sonic log. The processing steps to obtain the $Q \log$ are given in the following Section IV.

Figure 10 shows some of the relevant well logs through the reservoir interval. The far-right plot shows the lithology, with sand fractions in red, shale fractions in green, and carbonate fractions in blue. It is apparent that this reservoir is characterized by alternating sand and shale beds ranging in thickness from a few meters to $9 \mathrm{~m}(30 \mathrm{ft}$.) or more. Occasional carbonate beds of no more than a few feet in thickness are interspersed among the sand and shale beds. The middle-right plot shows the measured neutron porosity and a lithology-corrected porosity to take account that neutron tools overpredict porosity in shaley rock. 

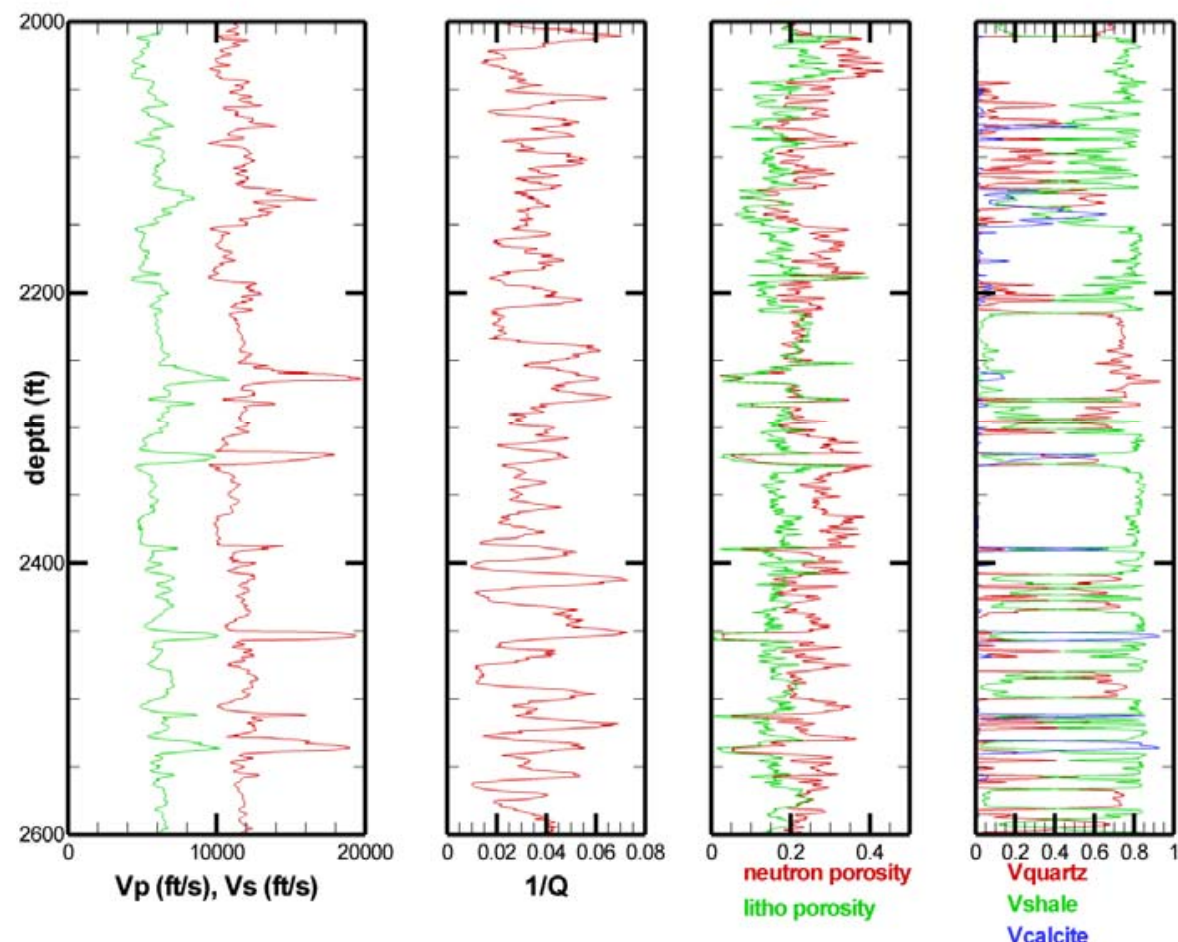

Figure 10. Measured and computed petrophysical properties in lower part of well \#3.

Since the primary lithological components are sandstone and shale, it is worth examining the relationships between different parameters in the context of sand vs. shale. For this purpose, we will rely on the lithology logs, with Vquartz values $>0.4$ indicating mostly sandstone rock, and Vshale values $>0.4$ indicating mostly shaley rock.

Figure 11 shows crossplots of porosity and permeability against Vp and Vs. There is a consistent trend of decreasing porosity with increasing velocity within each lithology type, and shaley rock velocities are generally lower than sandstone velocities. The Stonely permeability increases with increasing velocity in shaley rocks, but decreases with increasing velocity in sandstone rocks. Shaley rocks generally have Stonely permeabilities in the range of 0.01 to $10 \mathrm{md}$, while the sandstone rocks generally have Stonely permeabilities in the range of $10 \mathrm{mD}$ to 10 darcies. 


\section{Depth Range 2000 - $2600 \mathrm{ft}$ \\ $V_{\text {quart }}>0.4$
$V_{\text {shale }}>0.4$}
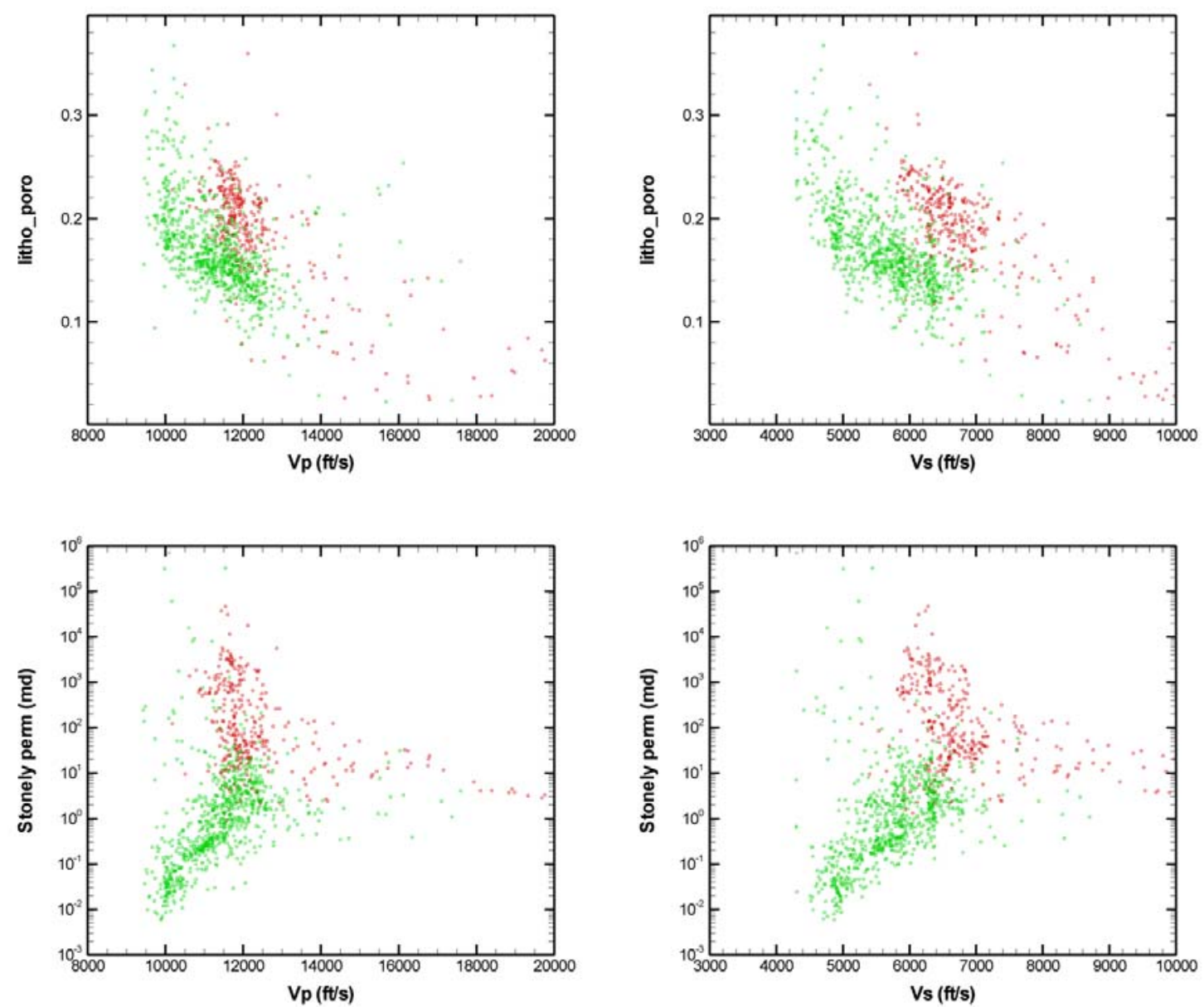

Figure 11. Crossplots of porosity and permeability with Vp and Vs for shaley and sandstone rock types.

One attribute of high interest is how $Q$ correlates with the other well logs. Figure 12 shows crossplots of the computed $Q^{-1}$ with velocity, permeability, and porosity. No strong correlation is observed between $Q$ and any of these quantities, although there is a slight trend toward higher attenuation at higher velocity. 

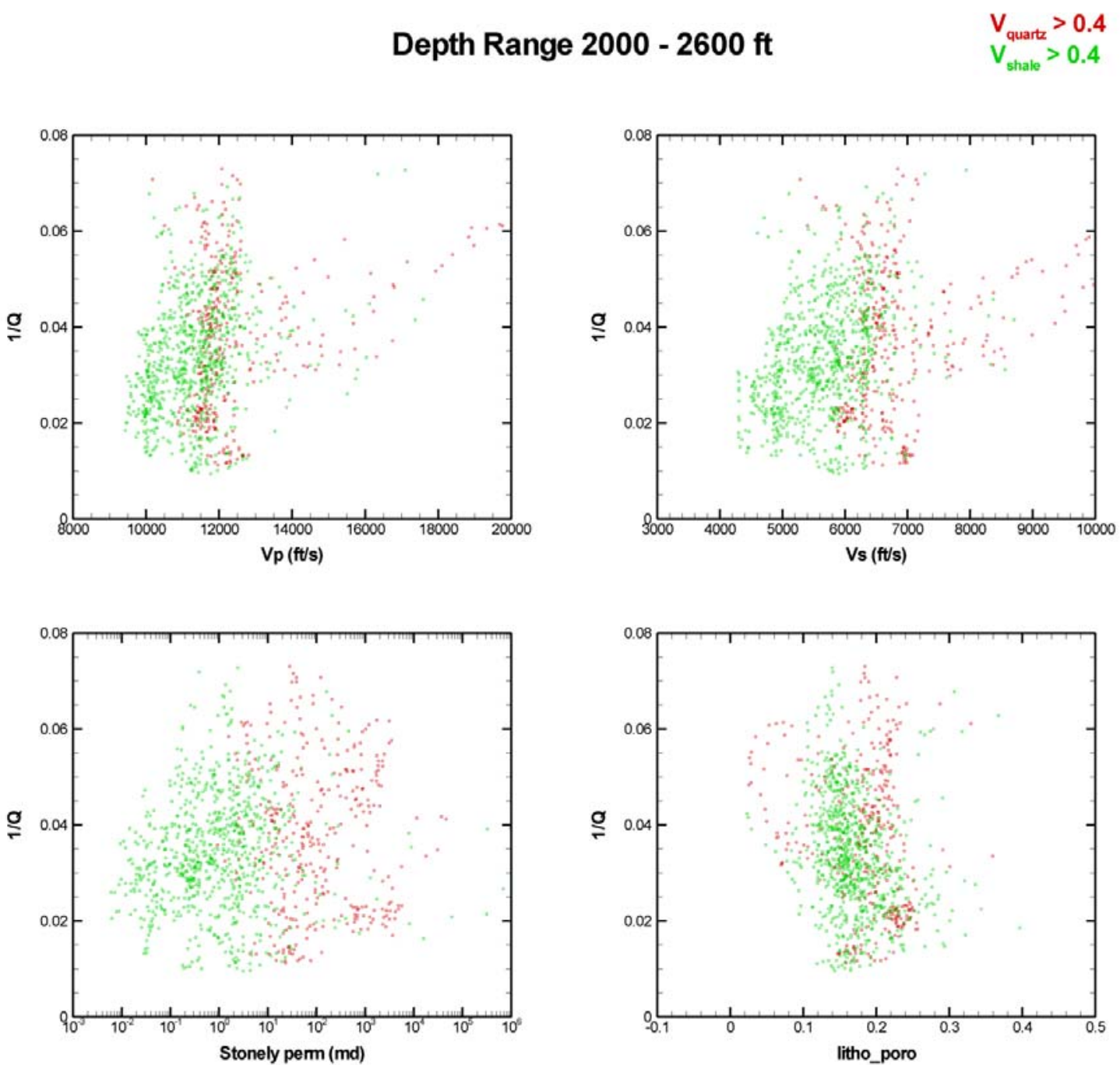

Figure 12. Crossplots of $Q^{-1}$ with Vp, Vs, porosity, and permeability for shaley and sandstone rock types.

Figure 13 explores the relationships between a few other pairs of parameters. Vs closely follows $\mathrm{Vp}$ in both lithologies, although the sandstone has a slightly lower $\mathrm{Vp} / \mathrm{Vs}$ ratio. There are no definite trends between $Q$ and rock composition, although sandstones appear to be slightly more attenuating on average than shales. Stonely permeability is positively correlated with the quartz fraction, as may be expected. 


\section{Depth Range 2000 - $2600 \mathrm{ft}$
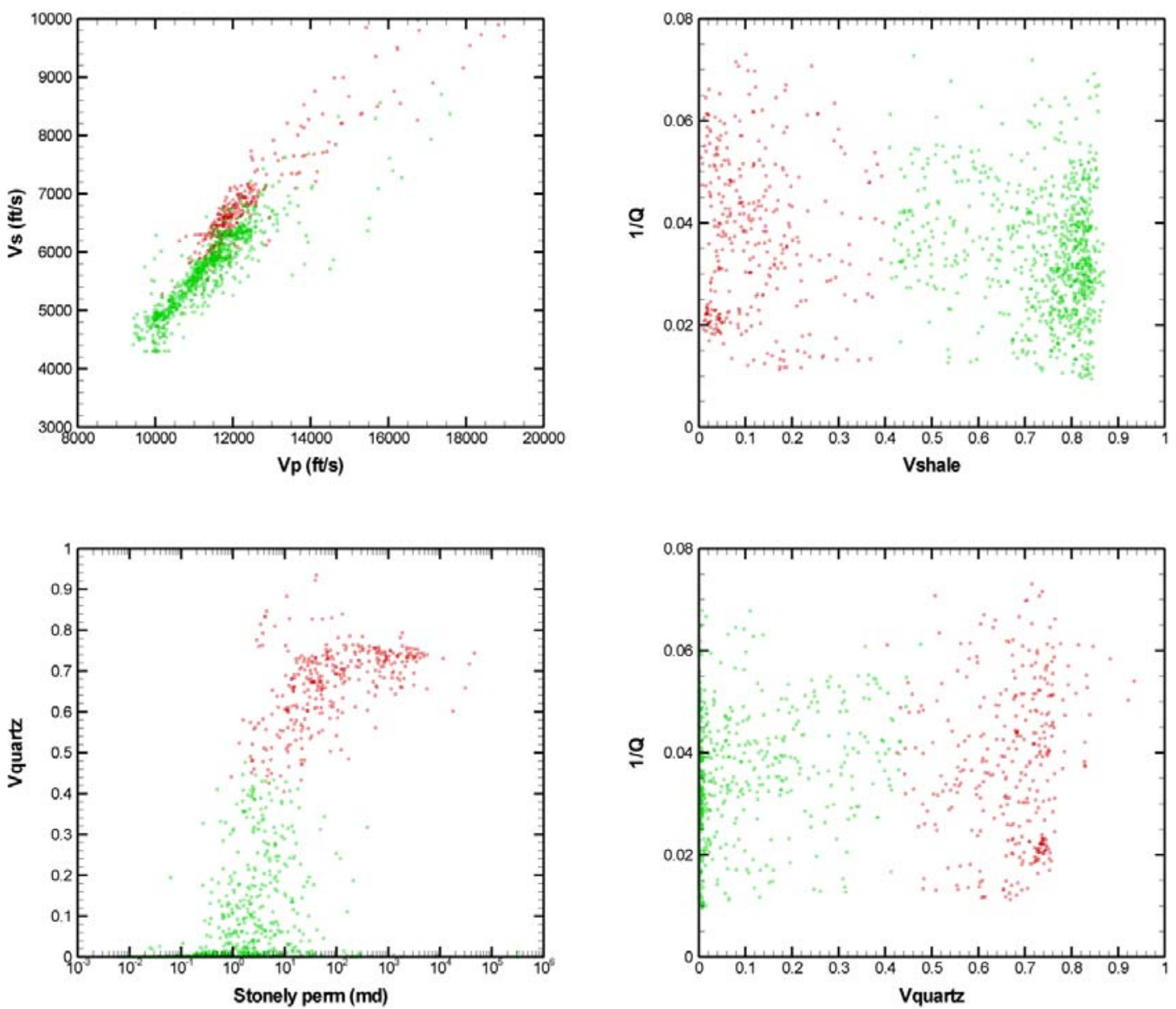

Figure 13. More crossplots from Waggoner well \#3.

\section{Synthetic logs}

In this task, we predicted bulk and shear moduli (and by extension Vp and Vs) for Waggoner wells 1, 2, and 3 using lithological composition logs. In this, we used the selfconsistent" model as described in Mavko's Rock Physics Handbook, section 4.8. This method takes as inputs the volume fractions of various constituents of a rock (including pore space), and the bulk and shear moduli of each constituent mineral or fluid. This is an iterative process in which the effective medium bulk and shear moduli of the composite rock are adjusted until the simultaneous composite equations are satisfied. For the Waggoner wells, the rock at each depth point is assumed to have four possible constituent materials: carbonate grains, quartz (sand) grains, shale grains, and water.

After experimenting with rock grain moduli values from the literature, we chose to perform an inversion for the rock grain moduli using the sonic log Vp and Vs from a small section of well \#3 [727-762 m (2385-2500 ft.)] as a training set. We iteratively adjusted the carbonate, quartz, and shale grain bulk and shear moduli (six parameters 
total) to minimize the mean-square deviation between sonic log bulk and shear moduli and the moduli predicted by the self-consistent model using the composition logs. Full water saturation was assumed. The bulk moduli of oil and water are very similar, so we did not expect oil saturation in the reservoir to significantly affect the results. Those results are presented in Table 6, along with typical values from the literature.

Table 6. Inverted and Typical Grain Moduli for Waggoner Rock Constituents

\begin{tabular}{|l|l|l|}
\hline & Computed Value & Typical Value \\
\hline Carbonate Grain Bulk Modulus & 58.8 & 70 \\
\hline Carbonate Grain Shear Modulus & 23.9 & 29 \\
\hline Quartz Grain Bulk Modulus & 29.0 & 37 \\
\hline Quartz Grain Shear Modulus & 16.7 & 33 \\
\hline Shale Grain Bulk Modulus & 31.4 & 21 \\
\hline Shale Grain Shear Modulus & 10.9 & 7 \\
\hline
\end{tabular}

Figure 14 shows the well $\log \mathrm{Vp}$ and $\mathrm{Vs}$ and the predicted $\mathrm{Vp}$ and $\mathrm{Vs}$ after inversion in the test region. The plot demonstrates a good match between calculation and observation, indicating that the inversion process was successful at determining the correct grain moduli to use for the self-consistent model in this zone. Having obtained a good set of grain moduli from a small section of one well, the next step is to apply these grain moduli and the self-consistent model to a wider zone of this well and to other wells where lithological composition logs are available.

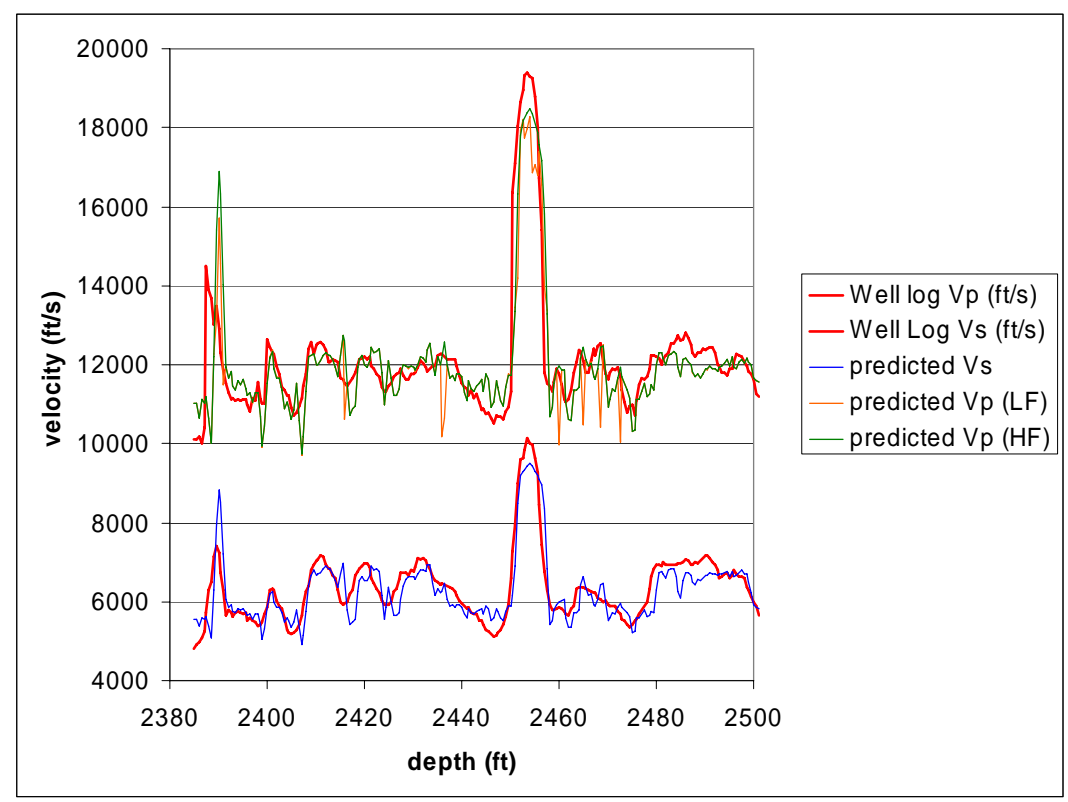

Figure 14. Comparison of well log Vp and Vs to predicted Vp and Vs after inversion for grain moduli. 
Figures 15 and 16 show that, in fact, the prediction model is broadly applicable. In Figure 15, we apply the model to depths of 457-793 m (1500-2600 ft.) in well \#3, with generally good results. In Figure 16, we apply the model to the reservoir depths of wells 1,2 , and 3 , again showing a good match over most depths.

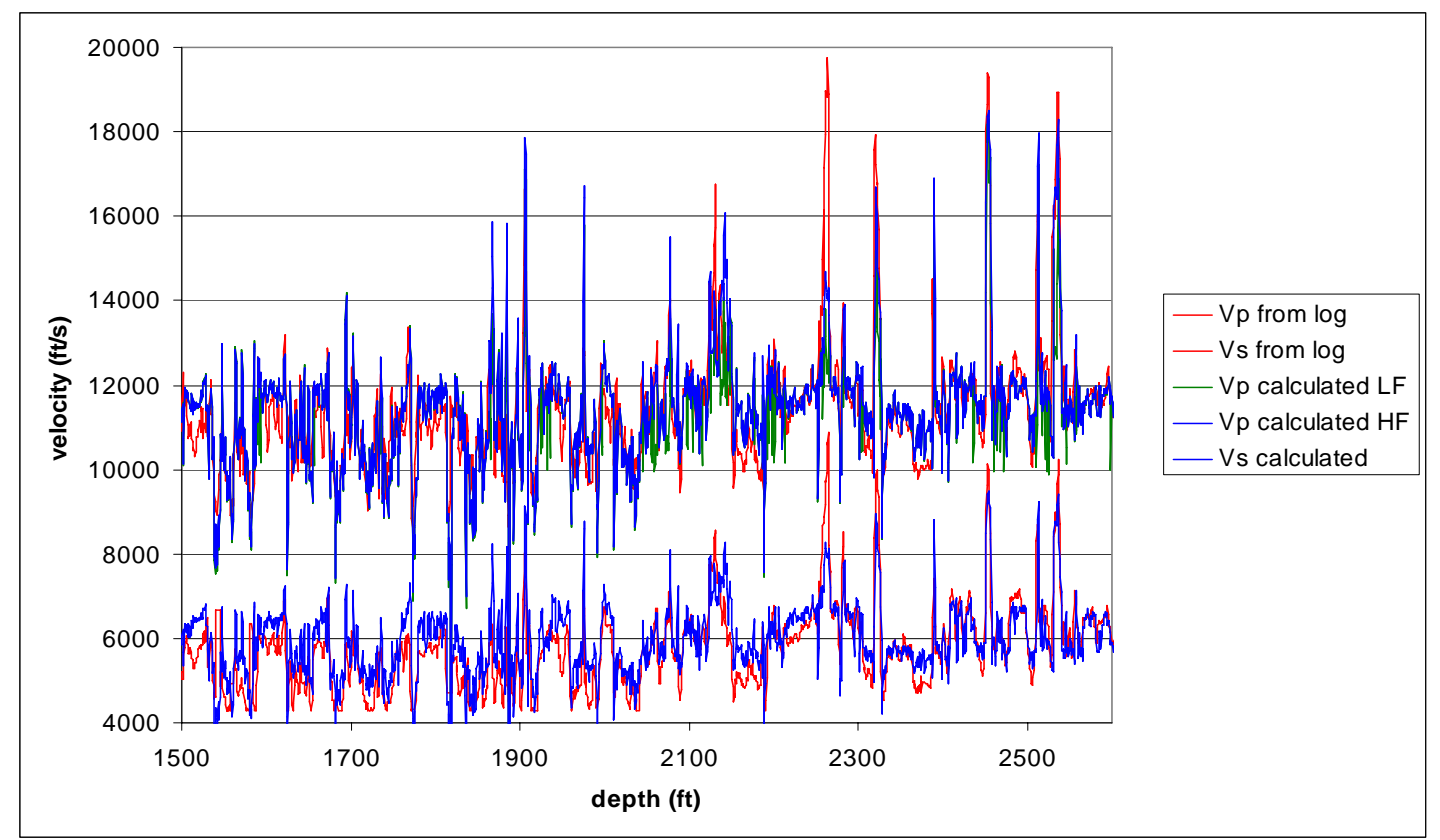

Figure 15. Comparison of measured and predicted Vp and Vs from well \#3 using grain moduli derived from a test region of 727-762 $\mathrm{m}$ (2385-2500 ft.) in depth. 

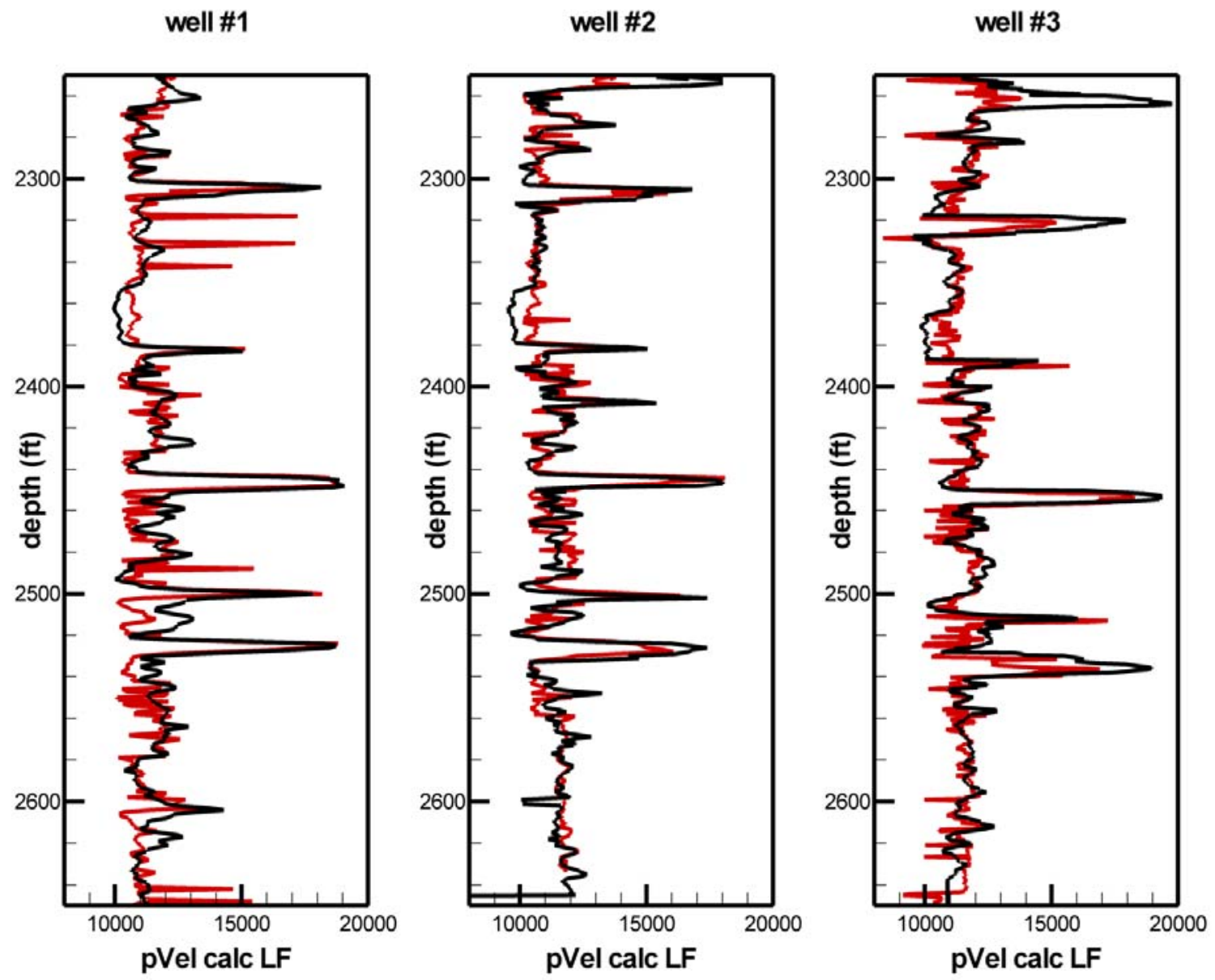

Figure 16. A comparison of measured (black) and predicted (red) Vp in three Waggoner wells, with predictions based on grain moduli derived from depths of 727-762 m (2385$2500 \mathrm{ft}$.) in well \#3. 


\section{EXTRACTION OF $Q$ LOG FROM WAGGONER DATA}

We applied the $Q$ processing algorithm to extract intrinsic attenuation $(1 / Q)$ from the head $P$-wave of a full waveform sonic $\log$. We found that the attenuation correlates with the lithology, including a sandstone zone partially saturated with oil and water. A comparison of $(1 / Q)$ with the micro-seismogram and the petrophysics of other well logs provide a way of evaluating the consistency of $Q$ at each borehole depth location. An analysis of the results explains the different attenuation anomalies found in the $Q \log$.

Figure 17 shows the primary (or first) ray and subsequent rays in the borehole. We estimate $Q$ from the sonic data and compare it with the lithology and other well logs to determine any possible correlation between $Q$ and rock and fluid properties; in particular, to determine what parameters the attenuation $(1 / Q) \log$ can capture to assist in the reservoir characterization.

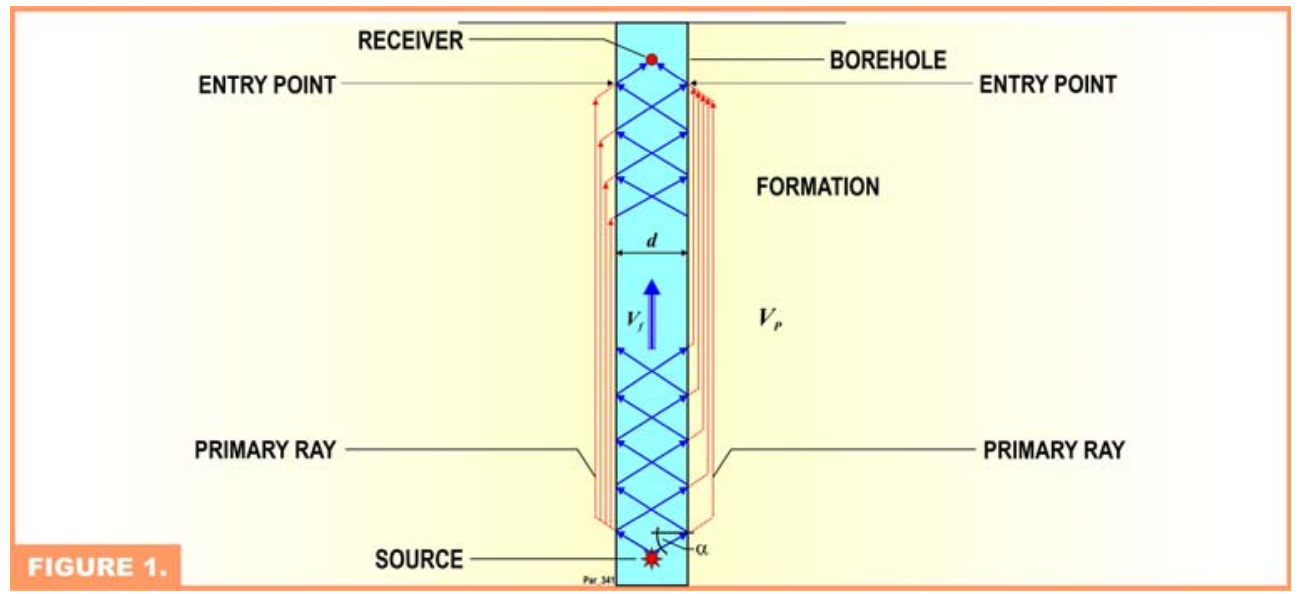

Figure 17. Examples of borehole head wave paths. $V_{P}$ is the P-wave velocity in the formation, $V_{f}$ is the acoustic wave velocity in the fluid, $d$ is the diameter of the borehole, and $\alpha$ is the critical angle. All wave fronts are cylindrically symmetric about the borehole axis, but to show more possible paths, we allow the left and the right wave fronts to be different.

The site geology consists of a sand-shale sequence. Based on well log data and cutting descriptions from three wells, the lithological units identified are sand (quartz), limestone (calcite), shale, and sandy shale (shale with a mixture of sand-to-silt-sized quartz or calcite grains). Shale laminations are present in all sandstones, and the limestone is a mudstone with very little porosity. There are two sandstone zones with moderate permeability and 20 percent porosity, one at $734-738 \mathrm{~m}(2409-2419 \mathrm{ft}$.) and one at 759-762 m (2489-2499 ft.) Both have a lamination of 10-20 percent clasts. 


\section{A. Application of the $Q$ Algorithm}

The reservoir is located in the Waggoner Ranch in northeast Texas. The three wells shown in Figure 18 intercepted a zone containing hydrocarbons at a depth of about $732 \mathrm{~m}(2400 \mathrm{ft}$.

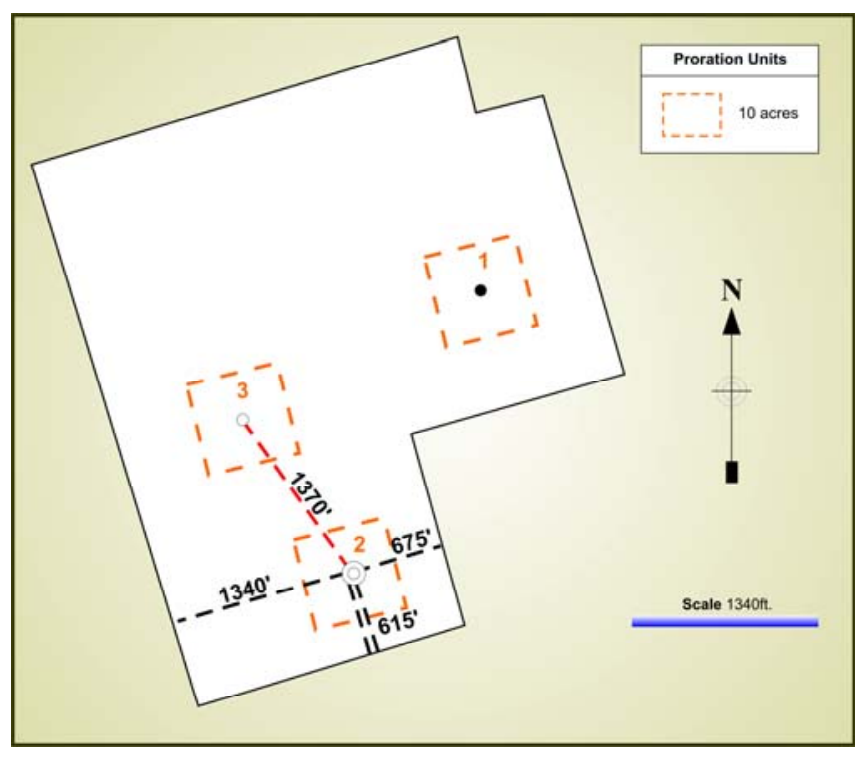

Figure 18. Location map for wells 1, 2 and 3.

Figure 19 shows a geological cross section between these three wells. The limestones are markers that helped to line up the lithological columns of the three wells. The sand containing oil saturation is about $20 \mathrm{ft}$. below the Upper Milham Marker. Only in Well \# 3 were acoustic full waveform data acquired. These data were recorded with an 8-receiver array. The source receiver offsets were 3.35, 3.5, 3.66, 3.8, 4.0, 4.12, 4.27, and $4.42 \mathrm{~m}(11,11.5,12,12.5,13,13.5,14$, and $14.5 \mathrm{ft}$.). The peak frequency of the sonic tool was about $10 \mathrm{kHz}$, and the $P$-wave velocity was typically $3.0-3.5 \mathrm{~km} / \mathrm{s}(9840-$ $11480 \mathrm{ft} / \mathrm{s}$ ) for this formation. With our code, we obtained results of $1 / Q$ for all possible receiver pairs with a spacing equal to or greater than $0.46 \mathrm{~m}$ (1.5 ft.). 

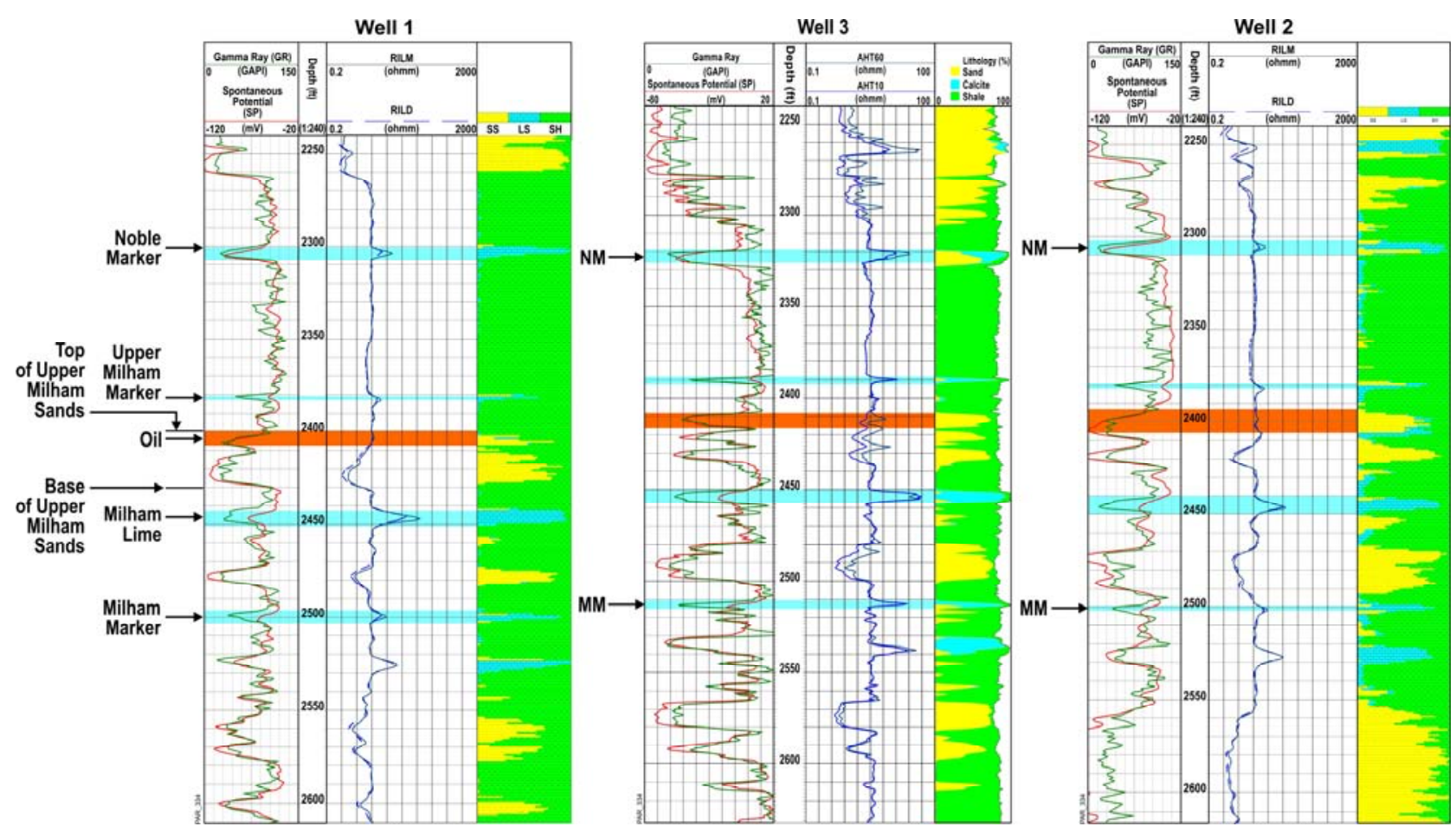

Figure 19. Geological cross section based on lithologic columns from wells 1, 2, and 3.

Figure 20 shows the $1 / Q \log$, velocity logs, and the microseismogram of receiver 1 from $671-762 \mathrm{~m}$ (2200 to $2500 \mathrm{ft}$.). We present two $1 / Q$ curves, one from receivers 1 and 5 [0.6 $\mathrm{m}(2 \mathrm{ft}$.) apart, blue line], and the other from receivers 1 and 7 [0.9 $\mathrm{m}(3 \mathrm{ft})$. apart, red line]. The depth for the processed $1 / Q$ is the midpoint between the receiver pair. On the other hand, a sonic waveform has been influenced by the average intrinsic attenuation from the source to the receiver. We therefore aligned the depth of $1 / Q$ with the midpoint between the source and the receiver of the waveform. In general, the $1 / Q$ $\log$ follows the regions of low and high amplitude waveforms. In the intervals of 674$681 \mathrm{~m}(2210-2235 \mathrm{ft}),. 692-701 \mathrm{~m}(2270-2300 \mathrm{ft}),. 710-718 \mathrm{~m}(2330-2355 \mathrm{ft}),. 720-729$ $\mathrm{m}(2360-2390 \mathrm{ft}),. 738-741 \mathrm{~m}(2420-2430 \mathrm{ft}$.$) , and 750-759 \mathrm{~m}(2460-2490 \mathrm{ft}$.$) , the$ attenuation is low or very low, and the amplitude of the head wave is high or very high. In the intervals of 683-689 m (2240-2260 ft.), 729-33 m (2390-2405 ft.), and 744-750 m (2440-2460 ft.), the attenuation is high, and the head wave amplitude is low.

We also analyzed the attenuation log for consistency by comparing it with other

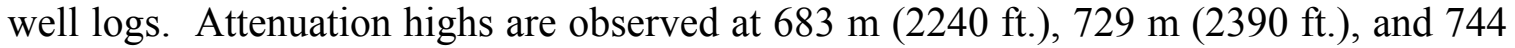
$\mathrm{m}(2440 \mathrm{ft}$.). They correspond to reservoir limestone markers. In addition, there is a high attenuation anomaly $(Q=16.5)$ in the interval at $734-738 \mathrm{~m}(2409-2420 \mathrm{ft}$.). This $Q$ corresponds to sand characterized by a permeability of $100 \mathrm{mD}$ and a porosity of 20 percent. This sand is partially saturated with oil and water and a gas phase, which results in high attenuation. Below this sandstone, in the interval at 734-762 m (2480-2500 ft.), there is another permeable sand of $184 \mathrm{mD}$ and 22 percent porosity. This sandstone is fully saturated with water and is associated with a low attenuation. The full saturation gives rise to a stiffer zone associated with a higher $Q$. 


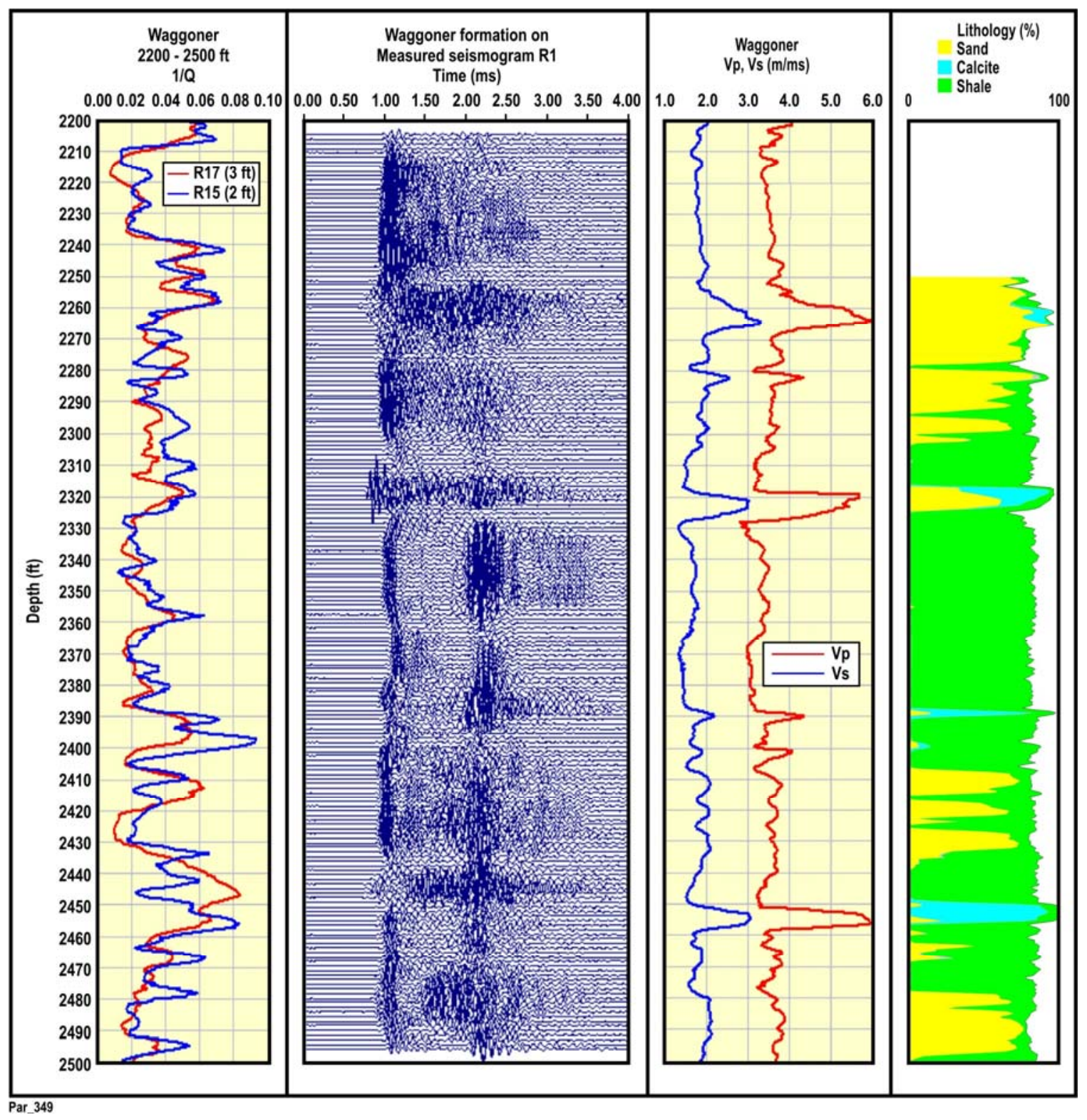

Figure 20. Comparison between $1 / Q, V_{P}$, and $V_{S}$ logs with acoustic full waveforms and the petrophysics of an oil reservoir. 
To further analyze the attenuation anomalies, we select the interval from 726-777 $\mathrm{m}$ (2380-2550 ft.) from Figure 20 that corresponds to the cored section. We compare the full waveform sonic data with their spectra, given in Figure 21.

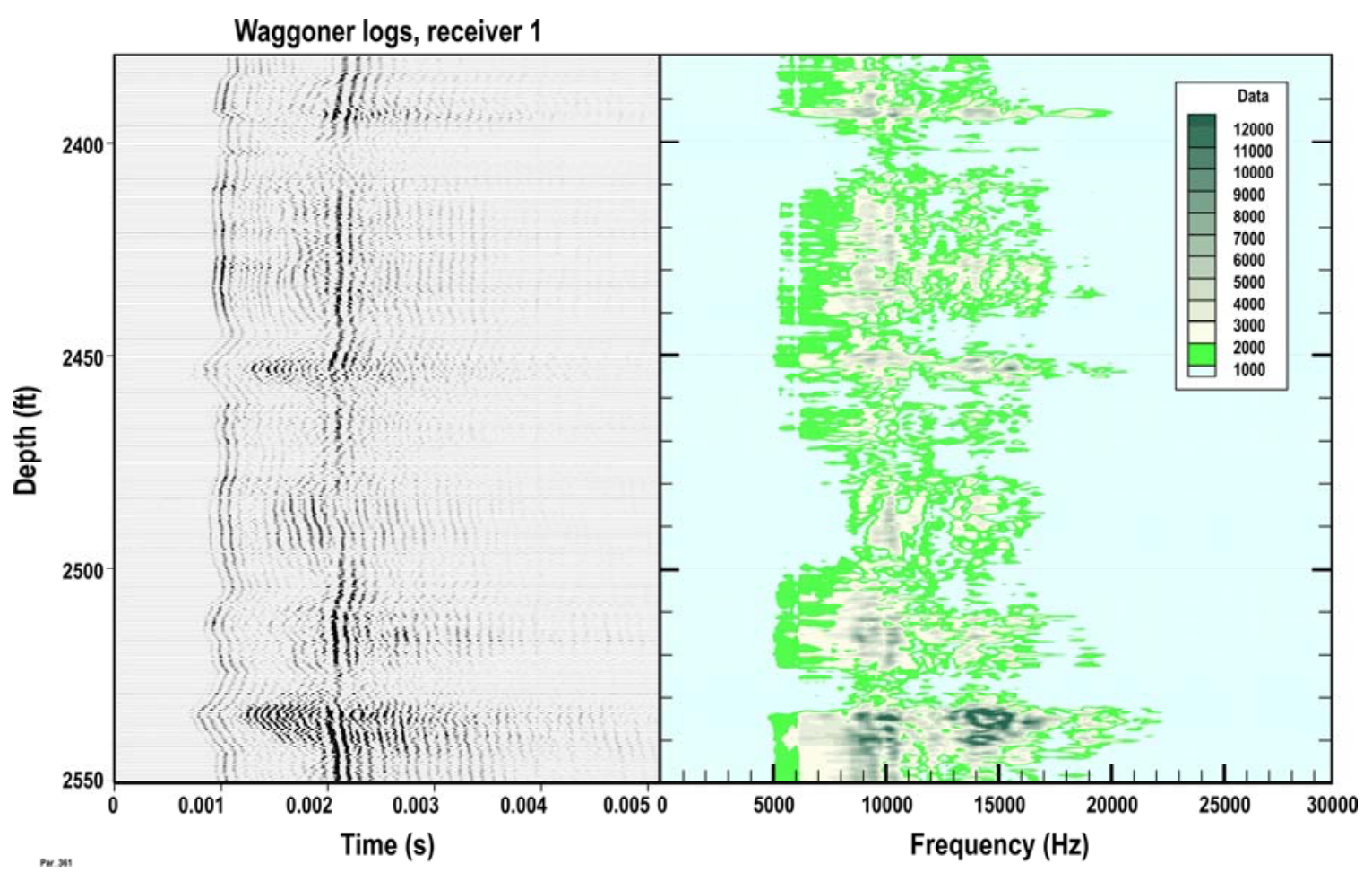

Figure 21. Comparison between acoustic signatures and their spectra in the interval depth of 726-777 m (2380-2550 ft.).

Both the spectra and the waveforms show reduced amplitude anomalies at depths

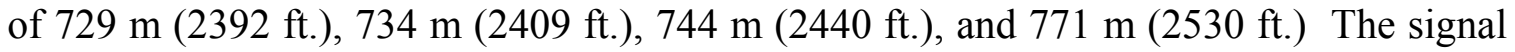

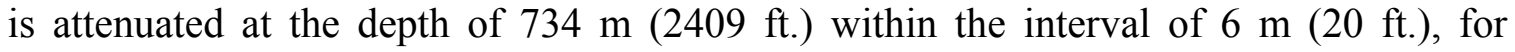
frequencies between 5000 and 20,000 Hz. On the other hand, the signal at $744 \mathrm{~m}(2440$ $\mathrm{ft}$.) is attenuated only for high frequencies (i.e., 10,000 to $20,000 \mathrm{~Hz}$ ), and it covers an interval of 3-6 m (10-20 ft.); the lower frequencies are less attenuated within the interval of $3 \mathrm{~m}$ (10 ft.). The signal is partially attenuated for frequencies of 5000 to $20,000 \mathrm{~Hz}$ in the interval of $3 \mathrm{~m}(10 \mathrm{ft}$.) at the depth of $771 \mathrm{~m}(2530 \mathrm{ft}$.).

The microseismogram and spectra show that the attenuation anomalies at $729 \mathrm{~m}$ (2392 ft.), $744 \mathrm{~m}$ (2440 ft.) and $771 \mathrm{~m}(2530 \mathrm{ft}$.) are associated with high-velocity limestone bounded by shale. These anomalies are characterized by low $Q$ values, and they occur near the transition zones between the bounding shale and the limestone.

The anomaly of interest at $734 \mathrm{~m}$ (2409 ft.), (the oil producing zone) does not present the same type of attenuation signature as that near limestone/shale transitions zones; in fact, at $734 \mathrm{~m}$ (2409 ft.) there is no limestone. In this interval Stoneley waves have been completely absorbed and body wave events are strongly reduced. Alternatively, the limestone/shale signatures contain both the head and Stoneley waves. 
We next relate the petrophysics to these signatures to characterize them and to understand their cause and origins.

\section{B. Petrophysical Analysis and Interpretation}

Figure 22 shows the lithology and the attenuation log with the resistivity and gamma ray/spontaneous potential logs for the interval between $726 \mathrm{~m}(2380 \mathrm{ft}$.) and 777 $\mathrm{m}(2550 \mathrm{ft}$.). The resistivity $\log$ accurately identifies the carbonate units, and the gamma ray/spontaneous potential logs identify the presence of sands and shale. The thin section descriptions integrated with the core analyses, logs, and core photographs allowed us to subdivide the core into seven petrophysical units.

\section{Well 3}

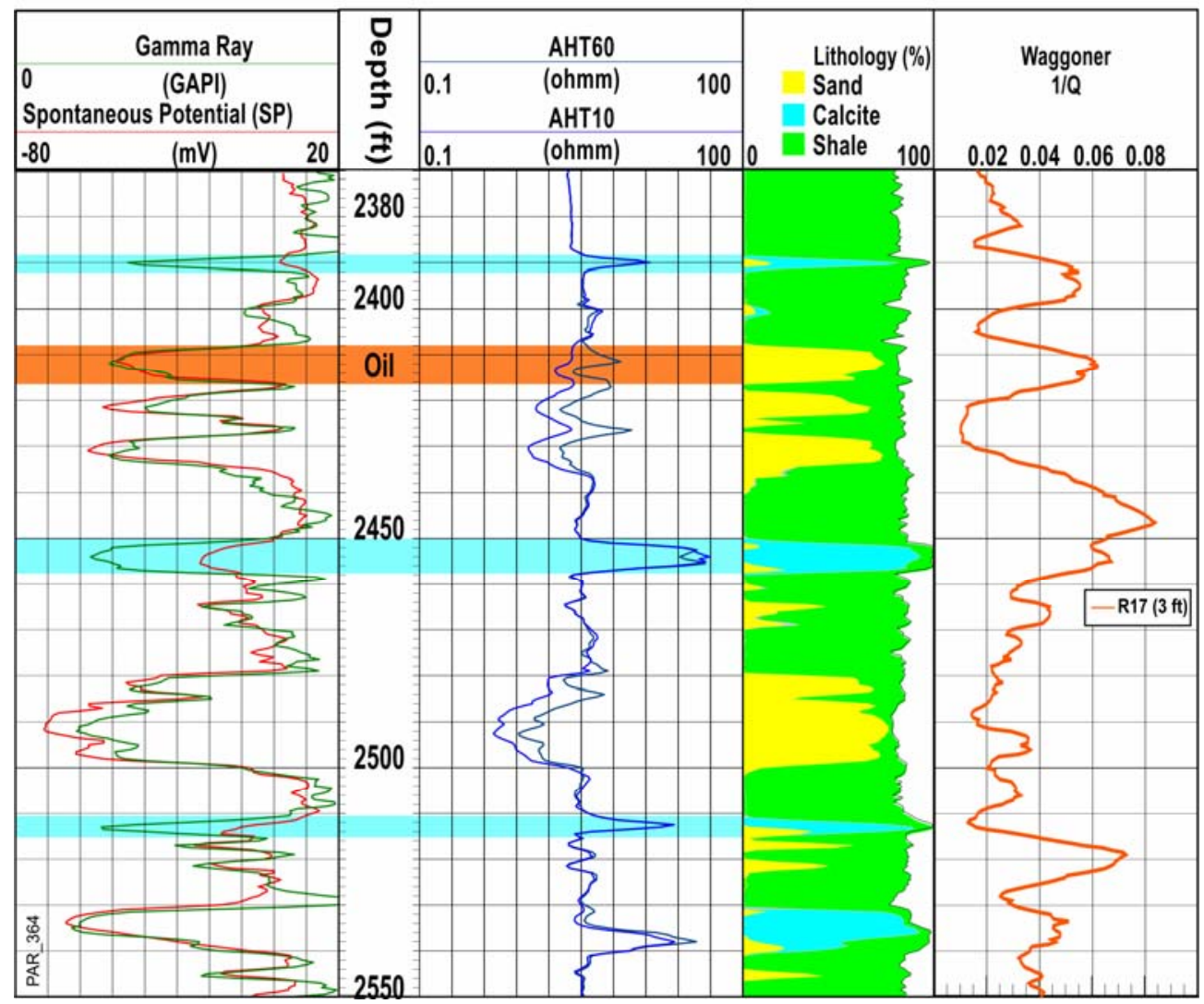

Figure 22. Comparison between standard logs (resistivity, gamma ray, spontaneous potential) and the $Q$-log to illustrate high attenuation zones versus lithology and fluid saturation.

The principal criteria for the units were lithology, permeability, porosity, and acoustic velocities. The seven units were identified 17 times in the cored interval. The names of the geological units and well logs are clearly shown in Figure 23. 


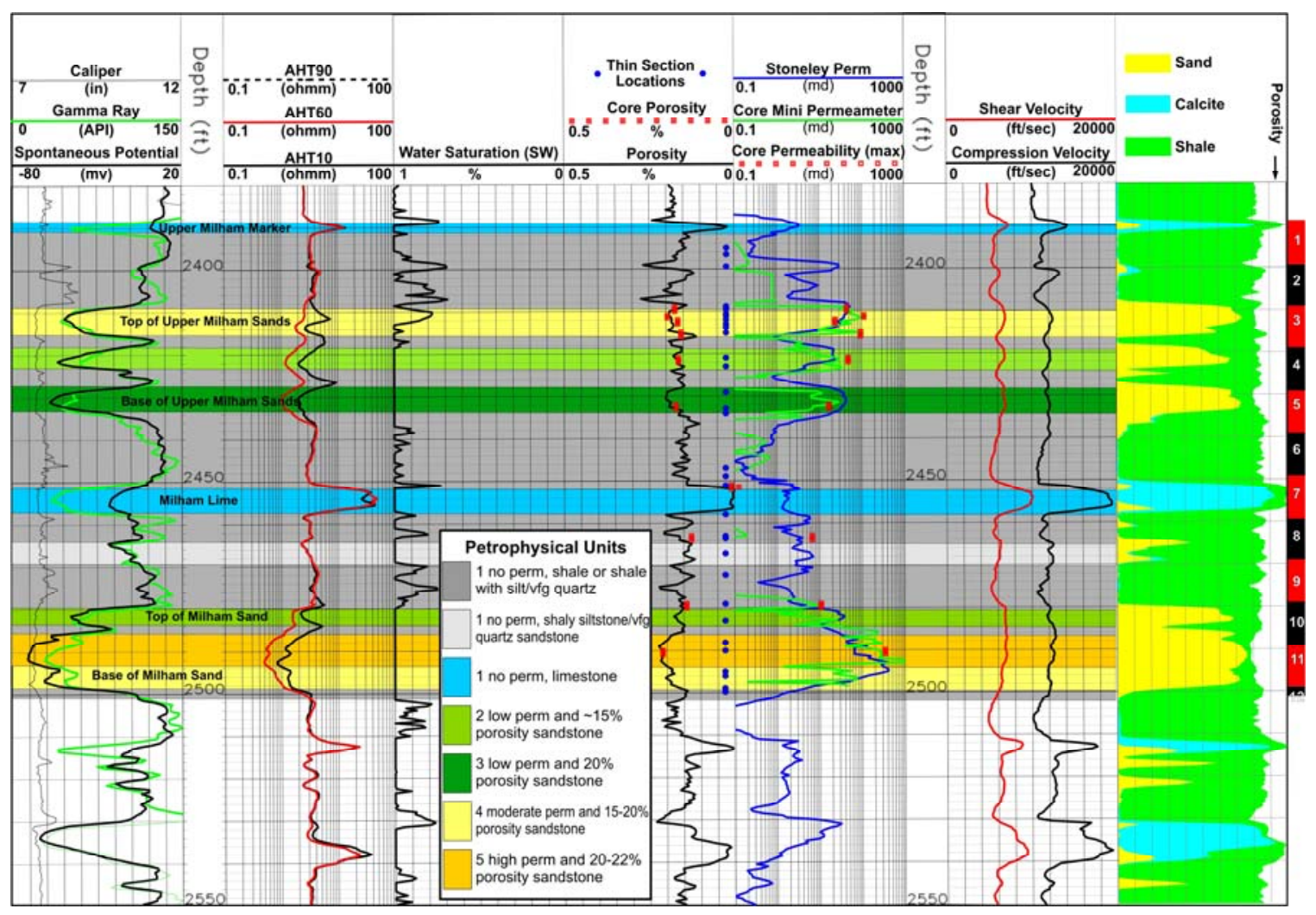

Figure 23. Petrophysical interpretation showing integration of core data and well logs, as well as the seven geological units derived from the core data analysis.

Standard well logs are compared with the lithology, permeability log, water saturation, and velocity logs. In Figures 24-26 we show examples of core intervals and thin sections at specific depths. We focus on the petrophysical units associated with the $Q$ values of interest.

\section{Unit 1 with no permeability}

In this unit, porosity is microscopic and a function of the shale content for the first two subdivisions. Log porosity values are therefore meaningless, since there is no effective porosity. There is very little pure shale. Most of it has a significant amount of silt or very fine-grained quartz. Less than $3 \%$ muscovite is present in some samples. Calcite fossil fragments and siderite nodules are present in a couple of intervals. Laminations, bioturbation, and rootlets are also present. Some intervals may be subaerially exposed "soil" horizons developed on levees or interdistributary bay fill. Shale laminations are present in all the sandstones. The limestone is a mudstone/packstone with very low, isolated, moldic porosity.

The limestone is characterized by an average P-wave velocity of $4910 \mathrm{~m} / \mathrm{s}$ $(16,107 \mathrm{ft} / \mathrm{s})$ and an S-wave velocity of $2532 \mathrm{~m} / \mathrm{s}(8306 \mathrm{ft} . / \mathrm{s})$. The shale unit has a P-wave velocity of $3540 \mathrm{~m} / \mathrm{s}(11,610 \mathrm{ft} . / \mathrm{s})$ and an S-wave velocity of $1819 \mathrm{~m} / \mathrm{s}(5965 \mathrm{ft} . / \mathrm{s})$. The 
P-wave velocity contrast between these two units is 28 percent, which suggests that shale bounded by a higher velocity limestone will act as a wave guide. A shale core sample with this characteristic in the interval at 744-747 m (2439-2449 ft.) is given in Figure 24; the shale volume is 85 percent. In this interval, the head wave event is reduced when the source moves from shale to limestone (see Figure 22). In general, this effect gives rise to scattering attenuation that, with intrinsic attenuation, produces the attenuation signal at the transition zone between limestone and shale.

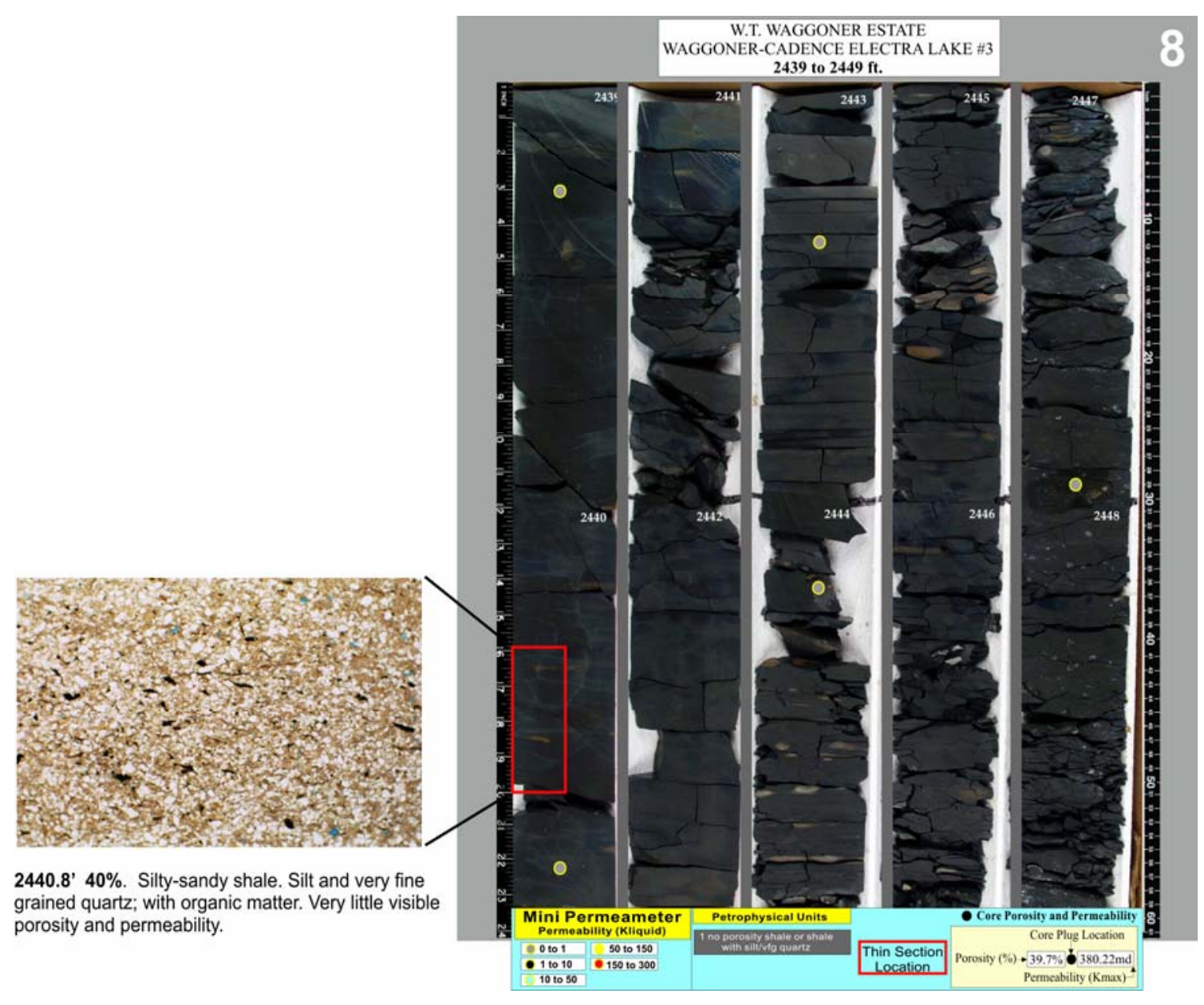

Figure 24. Core and thin sections from the interval at 744-747 m (2439-2449 ft.), characterized as low-velocity shale.

\section{Unit 4 with moderate permeability and $15-20 \%$ porosity sandstone}

There are two intervals with these petrophysical properties: 734-738 m (2409-2419 $\mathrm{ft}$; see Figure 25) and 759-762 m (2489-2499 ft.; see Figure 10). Both intervals have shale laminations and 10-20\% shale clasts. The intervals have different depositional environments. The 734-738 m (2409-2419 ft.) interval (Figures 25 and 22) has more 
shale laminations and is finer grained. Vertical and lateral permeability should be greatly restricted by the shale laminations. Oil production is from this interval. The P-wave and S-wave velocities of this interval are $3708 \mathrm{~m} / \mathrm{s}(12,161 \mathrm{ft} / \mathrm{s})$ and $2031 \mathrm{~m} / \mathrm{s}(6662 \mathrm{ft} / \mathrm{s})$, respectively, and the permeability is $100 \mathrm{mD}$. The intrinsic attenuation of this interval is associated with a $Q$ less than 20. The 759-762 m (2489-2499 ft.) interval (Figures 26 and 22) has considerable soft sediment deformation. This unit has P-wave and S-wave velocities of $3645 \mathrm{~m} / \mathrm{s}(11,956 \mathrm{ft} / \mathrm{s})$ and $1952 \mathrm{~m} / \mathrm{s}(6402 \mathrm{ft} / \mathrm{s})$, respectively, and a permeability of $184 \mathrm{mD}$. There is practically no velocity contrast between this unit and shale. The attenuation anomaly in this interval observed in the $Q$-log of Figure 22 is only intrinsic attenuation.

\section{Interpretation}

The core and thin section data from a depth of 759-762 m (2409-2419 ft.) shows that the partially oil-saturated sand contains shale laminations that diminish the fluid flow. The permeability in this case is approximately $100 \mathrm{mD}$, which is enough to allow fluid flow when a stress wave is propagated through this medium. The sand is partially saturated with water and some gas. This characteristic can reduce the wave amplitude. Figure 25 shows the core and the thin sections in the interval of 734-738 m (2409-2419 ft.). The shale laminations in the sand are clearly observed in the core. The thin sections illustrate the relationship between permeability and the degree of laminations in the core. Alternatively, the sand in the interval depth of 759-762 m (2489-2499 ft., Figure 26) has a different structure than the top sand and is more permeable. However, it is a fully water saturated sand. In this case, there is no gas and the stress wave is less attenuated. Since this sand is not near or intersected by a limestone unit, it does not produce resonances; it has its own signal characteristics. It is sand bounded by shale, with the sand being of slightly higher velocity than the shale. As a result, the seismogram and its spectra show a homogeneous pattern formed by a clean first event followed by a series of coherent events.

The above argument explains the high and low attenuation anomalies observed on the top and the bottom of Figure 22. Because of their permeability and fluid saturation, both are based on the change of pressure at the passage of an acoustic wave. To explain the high attenuation anomaly in the interval depth of 744-747 $\mathrm{m}$ (2440-2450 ft.), we use the core in Figures 24, 18 and 22 as a reference. The core shows pure shale, with no porosity and permeability, which is characterized as low velocity rock. This shale, bounded by a high-velocity limestone, produces a signature characteristic that contains resonances as observed in Figure 20. Based on the velocity log information, the P-wave velocity in the limestone interval between $747 \mathrm{~m}(2450 \mathrm{ft}$.) and $749 \mathrm{~m}(2458 \mathrm{ft}$.) is about $5581 \mathrm{~m} / \mathrm{s}(18,307 \mathrm{ft} / \mathrm{s})$, while the shale interval above this limestone unit has an average P-wave velocity of $11,047 \mathrm{ft} / \mathrm{s}$. Above this low velocity, 10 -ft. thick shale in the interval

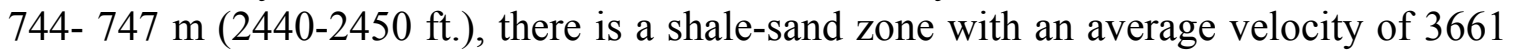
$\mathrm{m} / \mathrm{s}(12,008 \mathrm{ft} / \mathrm{s})$. This structure has a waveguide characteristic associated with resonances observed in the seismogram. Some of the energy will be trapped and lost in the waveguide, which will produce a higher attenuation near this low velocity zone. This is why a large attenuation anomaly is observed at a depth of $747 \mathrm{~m}(2450 \mathrm{ft}$.). 


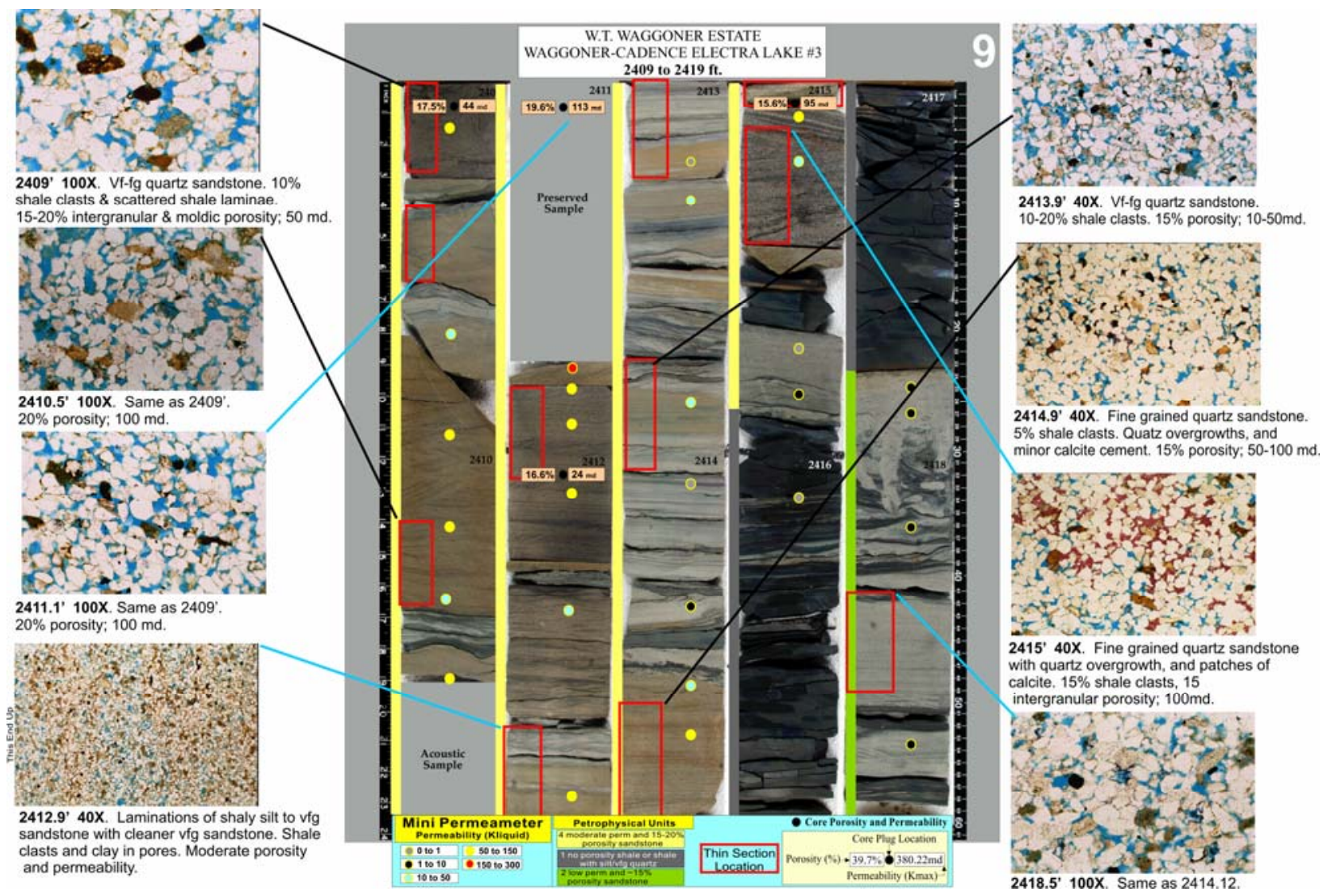

Figure 25. Core and thin sections from the interval at 759-762 m (2409-2419ft.), characterized as partially oil-saturated sand. 


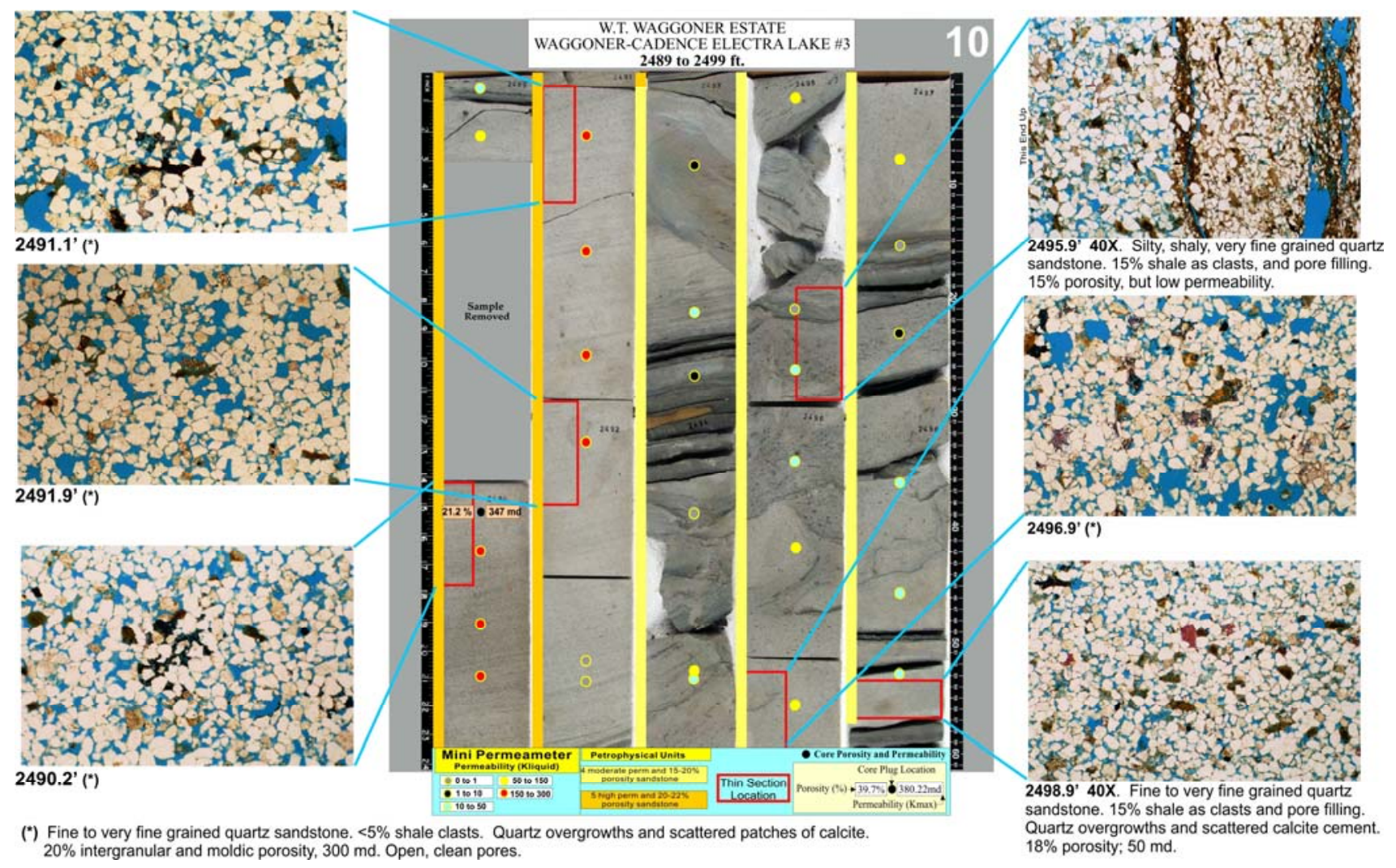

Figure 26. Core and thin sections from the interval at 759-762 m (2489-2499 ft.), characterized as fully water-saturated sand.

\section{Conclusions}

The $Q \log$ processed from the borehole sonic log demonstrates consistency with the microseismogram, including zones with high-velocity contrast between the limestone and shale and/or sandstones. The $Q \log$ has detected a zone that contains oil and water, which suggests that the fluid motion between the oil and the water in the presence of a gas phase reduces the acoustic energy in this interval. With the help of the petrophysics, the $Q \log$ allows us to evaluate the acoustic attributes in the microseismogram. It is well known that, after crossing a low velocity zone, the energy of a $\mathrm{P}$ wave is partially lost due to scattering. In this case, the observed, severely reduced $\mathrm{P}$ wave amplitude is due to both scattering and intrinsic $Q$. The results demonstrate that $1 / Q$ is an important log that, when integrated with other well logs, can discriminate between anomalies associated with lithology and those associated with oil and gas saturation. 


\section{A DOUBLE-POROSITY POROELASTIC MODEL TO RELATE P-WAVE ATTENUATION TO FLUID FLOW IN VUGGY CARBONATE ROCK}

Several self-consistent and empirical models for intrinsic and scattering attenuation were evaluated to explain the wave attenuation associated with rock/fluid types of reservoirs. Based on the data sets we used in this research program, double porosity models were effective in evaluating the $Q \log$ processing algorithm. The data set used for this study is from a south Florida aquifer. The geology and petrophysics of this site is given in Parra et al. 2003, a DOE Report that addressed the integration of NMR and acoustic well logs for the petrophysical characterization of the formation surrounding the well bore. The core data from the aquifer was used to simulate attenuation responses using the double porosity model presented in this section. This model is generalized in Section VI to simulate $Q \operatorname{logs}$ and used to explain the attenuation extracted from a sonic $\log$ from the south Florida aquifer.

In this section, we investigate the attenuation and fluid pressure due to mesoscopic flow in a double-porosity medium with a pore structure formed by a matrix and vuggy porosity. Attenuation curves of double porosity models are calculated using the theory developed by Pride and Berryman (2003). The Green's function for a volume source in a double porosity effective medium is developed to calculate fluid pressure spectral responses and seismic signatures based on typical south Florida vuggy carbonate rock properties.

\section{A. Problem Formulation}

When $\mathrm{P}$ waves excited by a seismic source propagate in an aquifer that is characterized by heterogeneities of different dimensions, such as variations in lithology and vuggy porosity, different regions respond with different fluid pressures. The associated fluid-pressure diffusion attenuates the wave energy. Three types of waves can be identified - a fast and a slow $\mathrm{P}$ wave and a shear wave. For the fast $\mathrm{P}$ wave, the pore fluid and porous matrix are compressed simultaneously; for the slow $\mathrm{P}$ wave, the porous matrix relaxes when the pore fluid is compressed.

In general, in a homogeneous fluid-filled porous medium, when the passing wave induces small fluid pressure gradients between regions of compression and extension, these gradients cause fluid flow relative to the solid. The fluid flow incurs viscous loss, resulting in a small attenuation of the passing wave. However, in real rocks this phenomenon is overwhelmed by local flow effects such those observed by Mavko and Nur (1975). These effects are caused by the pore fluid between regions of different compliances under the compression (or extension) induced by the passing wave. In an application given by Gurevich et al. (1997) for a medium composed by thin alternating layers of two poroelastic materials with different compliances, propagation of a $\mathrm{P}$-wave will squeeze the fluid from more compliant into the less compliant layers. This local flow of the pore fluid is accompanied by the viscous loss and results in the attenuation of the passing wave. On the other hand, in an aquifer when the P-waves compress an element containing mesoscopic heterogeneity, the different porosity types should respond with different changes in their fluid pressure. An internal equilibrium then takes place with fluid flowing from the more compliant high-pressure regions to the relatively stiff low- 
pressure regions. Such mesoscopic flow attenuates energy and affects the wave amplitude.

To calculate the pore pressure response due to a volume source we use the Green's function based on the effective Biot theory. We write the coupled system of equations directly from the constitutive relations given by Biot (1962). These are the total stress of the isotropic porous medium, the stress in the porous fluid, the momentum balance equation for total stress, and the generalized Darcy's law. Following Parra (1991) and Boutin et al. (1987), the coupled system of differential equations in the frequency domain for a volume source $(V)$ and a force $(F)$ is given by:

$\mu \nabla^{2} u+(\lambda+\mu) \nabla \nabla \bullet u+\omega^{2} \bar{\rho}-\bar{\alpha} \nabla p=-F$

$\theta \nabla^{2} p-\beta p-\bar{\alpha} \nabla \cdot u=-V$

where $u$ is the particle vector displacement of the solid, $p$ is the fluid pressure, and

$$
\begin{aligned}
& \bar{\rho}=\rho+\rho_{f}^{2} \omega^{2} \theta \\
& \bar{\alpha}=\alpha+\rho_{f} \omega^{2} \theta \\
& \lambda=K_{d}-2 \mu / 3 \\
& \quad \theta=\frac{j k(\omega)}{\eta \omega}
\end{aligned}
$$

where $\rho$ is effective bulk density, and $\lambda, \alpha$ and $\beta$ are effective constants related directly to the complex Biot constants $H, C$ and $M$ by:

$$
\begin{aligned}
& \beta=\frac{1}{M} \\
& \alpha=\frac{C}{M} \\
& \lambda+\mu=H-G-C^{2} / M .
\end{aligned}
$$

We also have the effective shear modulus relation, $\mu=G$, and the effective parameter, $\theta$, which includes the effective fluid inertia in the relative motion parameter, $\widetilde{\rho}$, i.e.,

$$
\theta=-\frac{1}{\omega^{2} \widetilde{\rho}}
$$

where $\widetilde{\rho}=-\eta /[j \omega k(\omega)], \quad k(\omega)$ is the complex frequency-dependent dynamic permeability, and $\eta$ is the fluid viscosity. 
The effective complex Biot constants $H, C$, and $M$ are defined in terms of the effective drained modulus of the composite $K_{d}$, the effective undrained bulk-modulus $K_{u}$, and the effective Skempton's coefficient $B$ as

$$
\begin{aligned}
& H=K_{u}+4 G / 3 \\
& C=B K_{u} \\
& M=\frac{B^{2}}{1-K_{d} / K_{u}} K_{u}
\end{aligned}
$$

where the effective complex functions $K_{d}, K_{u}$, and $B$ are given for double porosity materials by Pride and Berryman (2002).

The next step is to find the Green's function from Eq. (9). Parra (1991) solved this equation and developed a solution for the pressure source (or volume) using the Sommerfeld integrals. The fluid pressure in the frequency domain is given by

$$
p=\frac{V_{o}(\omega)}{4 \pi \omega^{2} \bar{\rho}}\left[\frac{\delta_{1}}{\alpha_{3}}\left(\frac{e^{j \lambda_{1} R}}{R}\right)-\frac{\delta_{2}}{\alpha_{3}}\left(\frac{e^{j \lambda_{2} R}}{R}\right)\right]
$$

where $R$ is the distance between the source and receiver, and the parameters $\lambda_{1}, \lambda_{2}$ are the wave numbers associated with fast and slow $\mathrm{P}$ waves traveling in an effective porous medium. The first and second terms in Eq. (13) are the fast and slow P waves, respectively. Wave numbers that control the phase velocity and attenuation of the wave traveling in the effective porous medium are summarized as follows:

$\lambda_{1}=[(b+\Delta) / 2]^{1 / 2}$

$\lambda_{2}=[(b-\Delta) / 2]^{1 / 2}$

where $b=k_{p}^{2}-\bar{\alpha}^{2} /(\lambda+2 \mu) \theta-\beta / \theta$

and $\Delta=\left[b^{2}+4 k_{p}^{2} \beta / \theta\right]^{1 / 2}$

in which $k_{p}^{2}=\omega^{2} \bar{\rho} /(\lambda+2 \mu)$.

In Eq. (13), the factors of the terms containing the exponentials are obtained from the relations given by

$\delta_{m}=\frac{\lambda_{m}^{2}-k_{p}^{2}}{2 \theta\left(\lambda_{1}^{2}-\lambda_{2}^{2}\right)} ;$

for $m=1,2$ and $\alpha_{3}=1 / 2 \bar{\rho} \omega^{2}$. 


\section{B. Results}

We use cores and well logs to construct and verify a double porosity model. Core data provide images of pore structure, such as matrix porosity and range of vug dimensions and physical properties (Figure 27). Velocity, porosity and density logs provide poroelastic properties. Permeability is derived from NMR well logs. These data are given in Parra and Hackert (2002) and Parra et al. (2002). Based on the solution given by Pride and Berryman (2002), we assume a spherical vug of radius $a$ surrounded by a homogeneous porous matrix.

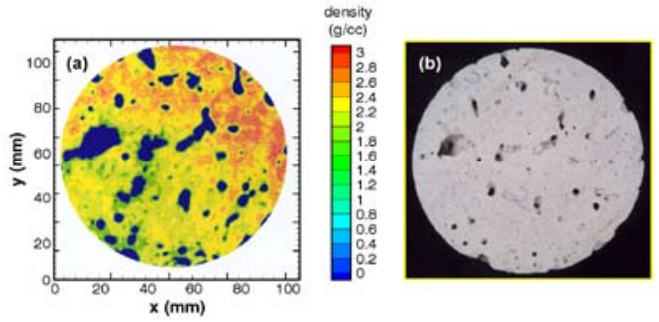

Figure 27. (a) Computed density from x-ray CT data, and (b) photograph of the end of the core.

The bulk modulus $K_{l}$ of the rock matrix in terms of the grain density, $\rho_{s}$, the P-and S-wave velocities of the dry skeleton $\left(v_{p}, v_{s}\right)$, and the porosity $\phi_{1}$, allow us to constrain the double porosity model by use of the well log data and the equation,

$K_{1}=\left(1-\phi_{1}\right) \rho_{s}\left(v_{p}^{2}-\frac{4}{3} v_{s}^{2}\right)$

From this data, we determine the following fluid and carbonate properties to construct the model:

$$
\begin{array}{lll}
K_{s}=72.2 G P & \eta=0.001 P \bullet s \\
G_{s}=38.6 G P & v_{p}=2276 \mathrm{~m} / \mathrm{s}(7465 \mathrm{ft} / \mathrm{s}) \\
\rho_{s}=2650 \mathrm{~kg} / \mathrm{m}^{3} & v_{s}=1036 \mathrm{~m} / \mathrm{s}(3398 \mathrm{ft} / \mathrm{s}) \\
v_{f}=1500 \mathrm{~m} / \mathrm{s}(4920 \mathrm{ft} / \mathrm{s}) & \phi_{1}=35 \% \\
\rho_{s}=1000 \mathrm{~kg} / \mathrm{m}^{3} & \phi_{2}=45 \%
\end{array}
$$

Alternatively, to simulate the bulk modulus of the vuggy structure we consider that soft materials are embedded in the interconnected pore matrix containing vugs. In this case we use the relationship (Pride et al., 2002),

$$
K_{2}=K_{s} \frac{\left(1-\phi_{2}\right)}{\left(1+c \phi_{2}\right)},
$$

Where $K_{s}$ is the modulus of the solid grain, $\phi_{2}$ is porosity, and $c$ is a consolidation parameter that represents the sediments and fluid inside the vugs (unconsolidated 
material). We use $c=200$. The effective compressional modulus (harmonic average of the $K_{1}$ and $K_{2}$ drained moduli) and the shear bulk modulus, $G$, of the double porosity medium can be obtained, respectively, from

$$
\begin{aligned}
& K=\frac{K_{1} K_{2}}{d_{2} K_{1}+d_{1} K_{2}}, \\
& G=(1-\phi) \rho_{s} v_{s}^{2},
\end{aligned}
$$

Where $\phi$ is the effective porosity of the double porosity medium given by

$$
\phi=\phi_{1} d_{1}+\phi_{2} d_{2} .
$$

The values of $d_{1}=0.963$ and $d_{2}=0.037$ are for a little sphere embedded in a stiff porous matrix with interconnected pore structure, as given in Pride and Berryman (2003).

Vug size varies in the pore matrix, with typical lengths from 1-6 cm $(0.03-0.20$ $\mathrm{ft})$. In the core in Figure 27, vugs are 15-20 mm (0.05-0.07 ft) in length. Based on NMR $\operatorname{logs}$, we find that permeability in the region of interest varies from 1-4 Darcies. Using these data we produce attenuation curves for vugs of dimensions $a=1,1.5,2$ and $3 \mathrm{~cm}$ $(0.03,0.05,0.066,0.10 \mathrm{ft})$, in an effective medium having a permeability of 3 Darcies, as shown in Figure 28(a). In this case the fluid flows easily from pore matrix to vugs and vice versa. Attenuation peaks are shifted to low frequency range as vug dimension increases. The mesoscopic heterogeneity (vug) of characteristic length $L=1.92 a$ equilibrates the pressure at a frequency

$f=D / L^{2}$

where $D$ is the pore-pressure diffusivity given by

$$
D=\frac{k}{\eta} \frac{B K}{\alpha}
$$

and where $k$ is permeability, $B$ is Skempton's coefficient, $K$ is drained bulk modulus, and $\alpha$ is the Biot-Willis coefficient of the medium. For given fluid and rock physical properties, frequency is inversely proportional to the characteristic length, $L$. This makes attenuation peaks shift downward with increasing vug size. The calculated equilibrium frequencies $\left(f_{\text {meso }}\right)$ for heterogeneities with radii of $1.0,1.5,2.0$, and $3.0 \mathrm{~cm}(0.03,0.05$, 0.066 , and $0.10 \mathrm{ft}$.) are $4446 \mathrm{~Hz}, 1976 \mathrm{~Hz}, 1112 \mathrm{~Hz}$, and $494 \mathrm{~Hz}$, respectively. To relate attenuation to fluid dynamics, we calculate the fluid pressure response due to a pressure source (or volume source) located $100 \mathrm{~m}$ from a pressure detector. The pressure response is calculated in MPa as a function of frequency, as shown in Figure 28(b), for $a=1$ to 3 $\mathrm{cm}(0.03-0.1 \mathrm{ft}$.). Each curve shows an increase in pressure to a maximum and then a sharp decrease to a minimum to values near the mesoscopic frequency $\left(f_{\text {meso }}\right)$. The plots show that as vug dimension increases, pore pressure decreases in the frequency range of 
3-1000 Hz. The pressure peaks are shifted to the lower frequencies as vug size increases. An internal equilibrium takes place as the pulse deforms the pore space, and fluid tends to flow from compliant high-pressure regions to relatively stiff low-pressure regions. Such mesoscopic flow reduces wave amplitude. In fact, the maximum pressure for each correlation length (vug dimension) occurs at a frequency less than the corresponding mesoscopic frequency and at lower attenuations. Lower frequency corresponds to long periods for a traveling pressure pulse. Long periods (low frequencies) provide sufficient time for the flow to occur during one-half period of the wave cycle. Thus, the fluid pressure will equilibrate and the rock will be in a relaxed state.

At high frequencies (short periods) there is not enough time for flow to occur, and the rock is in an unrelaxed state, thus, the rock is stiffer at high frequencies and has higher wave velocities. At intermediate frequencies, interactions between fluid and solid is at a maximum, and a maximum attenuation occurs. In this case, the wave amplitude is reduced practically to zero.

In Figure 28(b) we observe that in the frequency range between $3-750 \mathrm{~Hz}$, the spectra exhibit rapid oscillations. This part of the spectra captures the slow P-wave motion due to propagation of a pulse in the fluid (water) of the aquifer when the matrix is relaxed (or in state of equilibrium). Oscillations in the spectrum arise from phase differences in the slow and fast waves; these rapid oscillations occur for each of the pressure peaks. This suggests that the slow wave can be excited at frequencies equal to or less than the mesoscopic frequency.

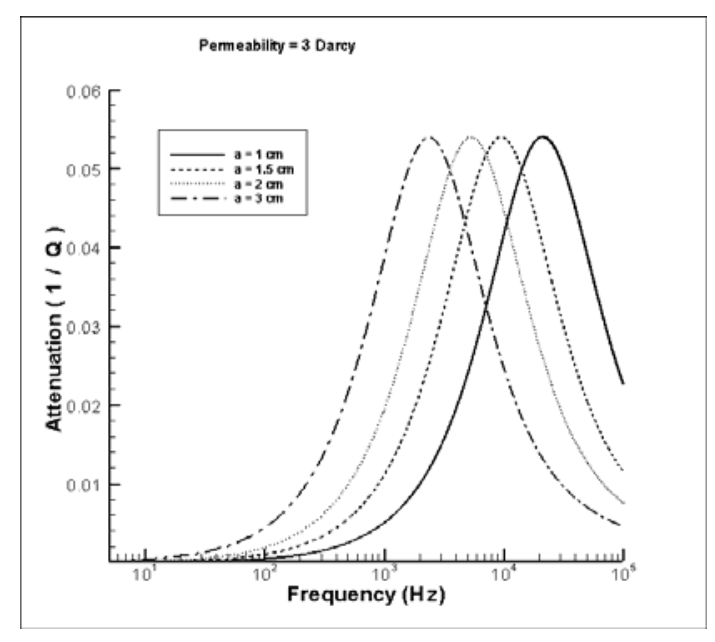

(a)

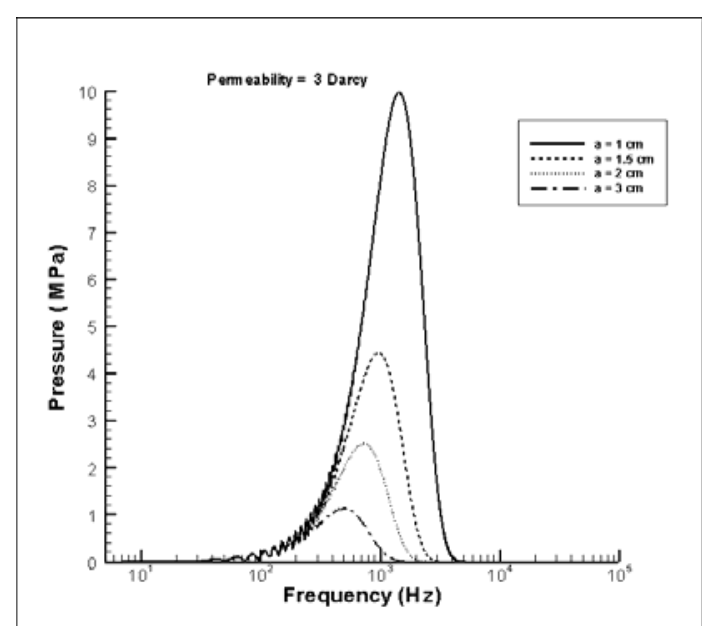

(b)

Figure 28. (a) Attenuation curves, and (b) pressure spectral response illustrating the length scale effect on attenuation and fluid pressure.

To analyze this effect in more detail, we calculate the spectrum of slow $\mathrm{P}$ waves only by using the second term of Eq. (13). Figure 29 shows the spectra of slow P waves for $a=1-3 \mathrm{~cm}(0.03-0.1 \mathrm{ft})$. The curves show pressure sensitivity to vugs in the pore matrix; the fluid pressure is sensitive to vugs at frequencies greater than $100 \mathrm{~Hz}$. For frequencies less than approximately $100 \mathrm{~Hz}$, the fluid pressure amplitude should remain unchanged. 
The high permeability of the carbonate aquifer (easy flow) provides the right conditions for part of the P-wave energy to compress the fluid and excite slow waves. This phenomenon can also be seen in the time domain. A pressure source having a peak source frequency of $300 \mathrm{~Hz}$ excites fast and slow $\mathrm{P}$ waves that are recorded by a pressure detector at a horizontal distance of $100 \mathrm{~m}$. The waveforms are calculated for $a=1,1.5,2$, and $3 \mathrm{~cm}(0.03,0.05,0.066,0.10 \mathrm{ft}$.) vugs, in an effective medium of permeability $=3$ Darcies (see Figure 30). In the traces, the fast P wave arrives with a velocity of $2450 \mathrm{~m} / \mathrm{s}$ $(8036 \mathrm{~m} / \mathrm{s})$, which agrees with the P-wave velocity log. The second event observed in each trace is the slow $P$ wave that travels with a velocity of $1250 \mathrm{~m} / \mathrm{s}(4100 \mathrm{ft} . / \mathrm{s})$. As expected when vug size increases, the amplitude of the fast $\mathrm{P}$ wave is more attenuated, and the amplitude of the slow P wave remains approximately the same. It is not affected by vug size as long as permeability does not vary.

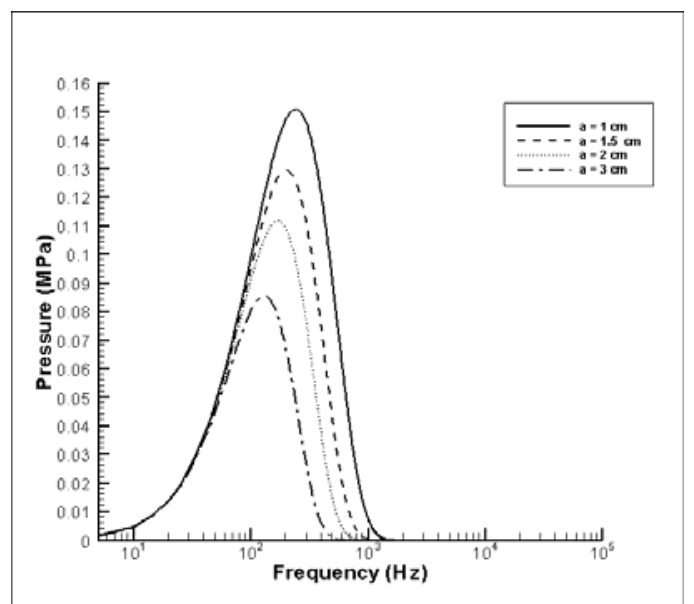

Figure 29. Slow-wave pressure spectral responses illustrate the length scale effect on fluid pressure.

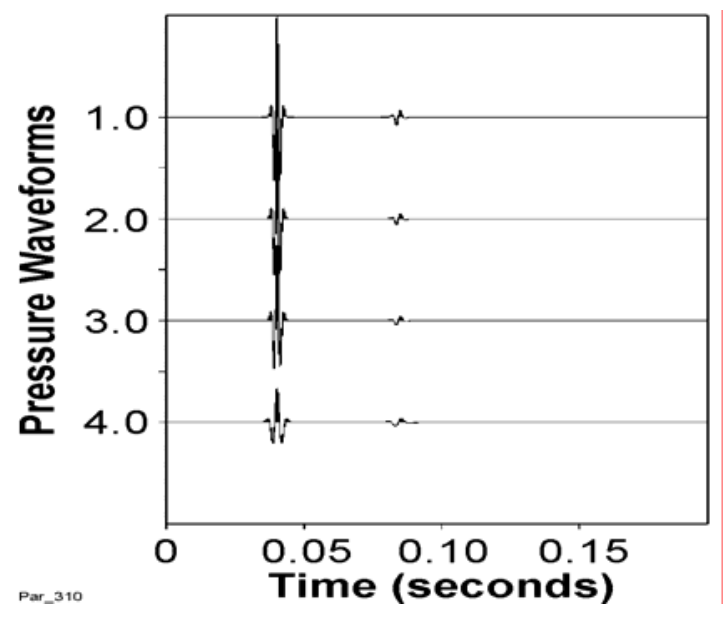

Figure 30. Pressure waveforms illustrating the length scale effect on fast and slow Pwave events. Waveforms correspond to vug sizes of $1,1.5,2$, and $3 \mathrm{~cm}(0.03,0.05,0.066$, $0.10 \mathrm{ft}$ ), in a matrix of 3-Darcy permeability. 
In the second example given in Figure 31, we examine how permeability affects interconnected vugs. We assume that fluid will flow from the vugs to the pore matrix with the same permeability for a given length scale. We vary permeability from 1-3 Darcies for a vug size of $2 \mathrm{~cm}$. As in the previous example, the mesoscopic heterogeneity has a characteristic length $[a=2 \mathrm{~cm}(0.066)]$, and the fluid pressure equilibrates when the frequency is $f=D / L^{2}$. According to Eq. (20), pore pressure diffusivity $D$ is proportional to permeability, which causes the mesoscopic attenuation peaks to shift upward to higher frequency with increasing permeability for a given heterogeneous medium, as shown in Figure 31(a). For low frequencies, fluid pressure attenuates more as permeability decreases, as in Figure 31(b). This means that at lower frequency (long periods), fluid motion (from vugs to pore matrix) becomes unrelaxed in low permeability environments, and the presence of slow waves is diminished (i.e., less fluid pressure decreases the generation of slow waves). In a more permeable medium (e.g., 3 Darcies), fluid pressure increases and the slow wave can be excited and will be less attenuated.

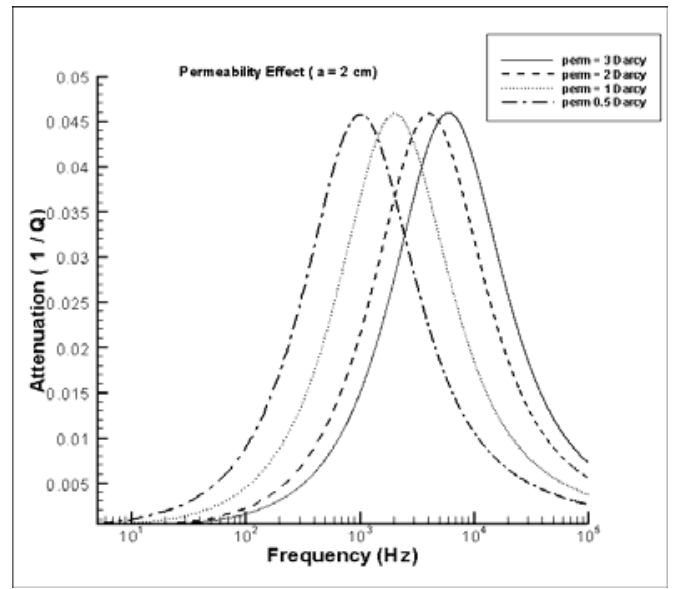

(a)

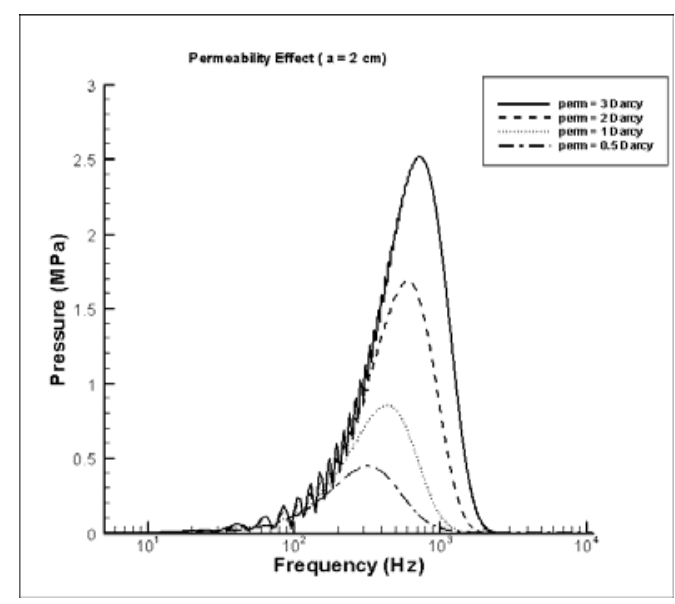

(b)

Figure 31. (a) Attenuation curves, and (b) pressure spectral response illustrate the effect of permeability effects on fluid pressure attenuation.

Finally, we calculate synthetic seismograms for this model to show the effect of fast and slow $\mathrm{P}$ waves as a function of permeability. Each trace of Figure 32 represents different permeability. The waveforms were produced for a peak source frequency of 300 $\mathrm{Hz}$. At this frequency, both waves are sensitive to permeability. For frequencies $\leq 100 \mathrm{~Hz}$ (see Figure 31), as permeability decreases, the amplitude of the fast $\mathrm{P}$ wave remains about the same, but slow wave amplitude decreases as permeability decreases. 


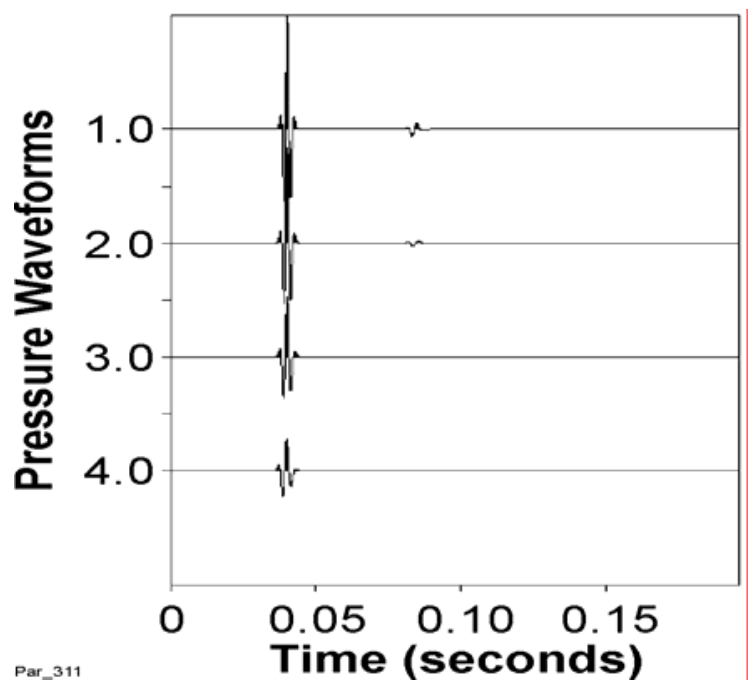

Figure 32. Pressure waveforms illustrating permeability effects on fast and slow P-wave events. Waveforms are for 0.5, 1, 2, and 3 Darcy permeability, with a vug of radius $a=2 \mathrm{~cm}(0.066 \mathrm{ft}$. $)$

\section{Conclusions}

The effective double-porosity model was successfully adapted to the dynamic poroelastic wave equation to simulate $\mathrm{P}$-wave and slow wave attenuation and spectral responses due to poroelastic fluid flow in vuggy carbonate rocks. We demonstrated that $\mathrm{P}$-wave attenuation is sensitive to the pore structure of the carbonate rock formation. We found that the slow wave has a characteristic spectral signature that can be detected with seismic techniques in formations having interconnected vuggy porosity. This suggests that the hydraulic properties of vuggy zones can be inferred from P-wave attenuation measurements. The present solution allows us to relate the amplitude of fluid pressure oscillations to the attenuation of the wave motion, thus enhancing our understanding of coupling between the poroelastic fluid-flow and the vuggy porous matrix when conducting monitoring surveys. 


\section{MODELING AND INTERPRETATION OF $Q$ LOGS IN CARBONATE ROCK}

As demonstrated in Section $\mathrm{V}$, attenuation is sensitive to vuggy and matrix porosities in a carbonate aquifer. To understand how the petrophysics relates to attenuation, we first use velocity and densities versus porosity relationships based on core and well log data to determine the matrix, secondary, and effective bulk moduli. The attenuation model requires the bulk modulus of the primary and secondary porosities. We use a double porosity model described in Section $\mathrm{V}$ to investigate attenuation at the mesoscopic scale. Thus, the secondary and primary porosities in the aquifer should respond with different changes in fluid pressure.

\section{A . Data Analyses}

The south Florida well is a test site for an aquifer storage and recovery system. The formation of primary interest is a vuggy, high-porosity, high-permeability carbonate bounded above by sand, shale, and carbonate mudstones; and below by lower-porosity, lower-permeability chalky carbonate. The attenuation algorithm was used to estimate $Q$ from a monopole sonic log recorded in well PBF10 in south Florida (Parra et al., 2004). In Figure 33, we compare acoustic attributes in the micro-seismogram between depths of 305-381 m (1000-1250 ft.).
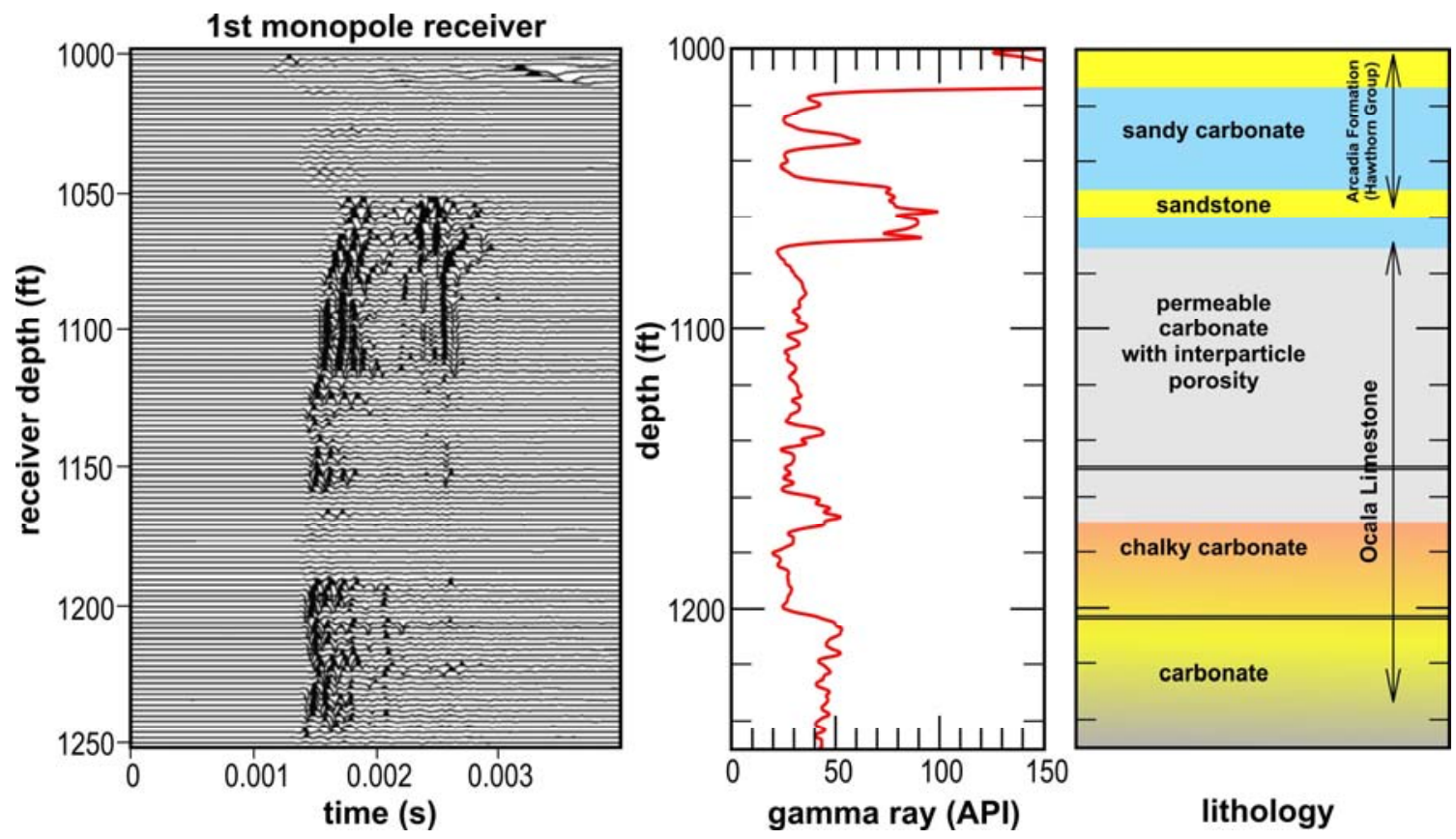

Figure 33. Comparison of sonic waveforms and lithology at the south Florida aquifer

This figure shows regions where acoustic waves are strongly attenuated. The highly attenuated attributes between depths of 305-323 m (1000-1060 ft.) are due to sandy carbonates and two thin sandstone layers. The pores are not well connected due to 
the presence of clay and quartz. These two minerals and the calcite that is also present comprise a mixture that, on average, forms a rock type with much lower bulk moduli than that formed by calcite alone. The high amplitude attribute observed between depths of 323 and $354 \mathrm{~m}$ (1060 and $1160 \mathrm{ft}$.) is associated with a vuggy carbonate with interconnected porosity. This interval is a vuggy permeable carbonate rock formation characterized by a pore structure formed by interconnected vugs with the pore matrix that allow fluid to flow in this double porosity medium. This interpretation was supported by high permeability measurements at the core and borehole scales (Parra et al., 2001). The next highly attenuated attribute between depths of 354-366 m (1160-1200 ft.) is due to a chalky carbonate. This unit has high porosity and low permeability, and it has a muddy texture with a vuggy porosity of less than 10 percent. The pore structure has collapsed to yield a medium with absorbing characteristics. The last zone corresponds to a much more permeable carbonate with higher vuggy porosity and an interconnected pore matrix.

The $Q$ processing algorithm automatically takes into account scattering and geometrical spreading, as well as borehole effects. The observed attenuation (in gray) is presented along with the vuggy and matrix porosity logs in Figure 34, which shows the statistically predicted attenuation (black line).
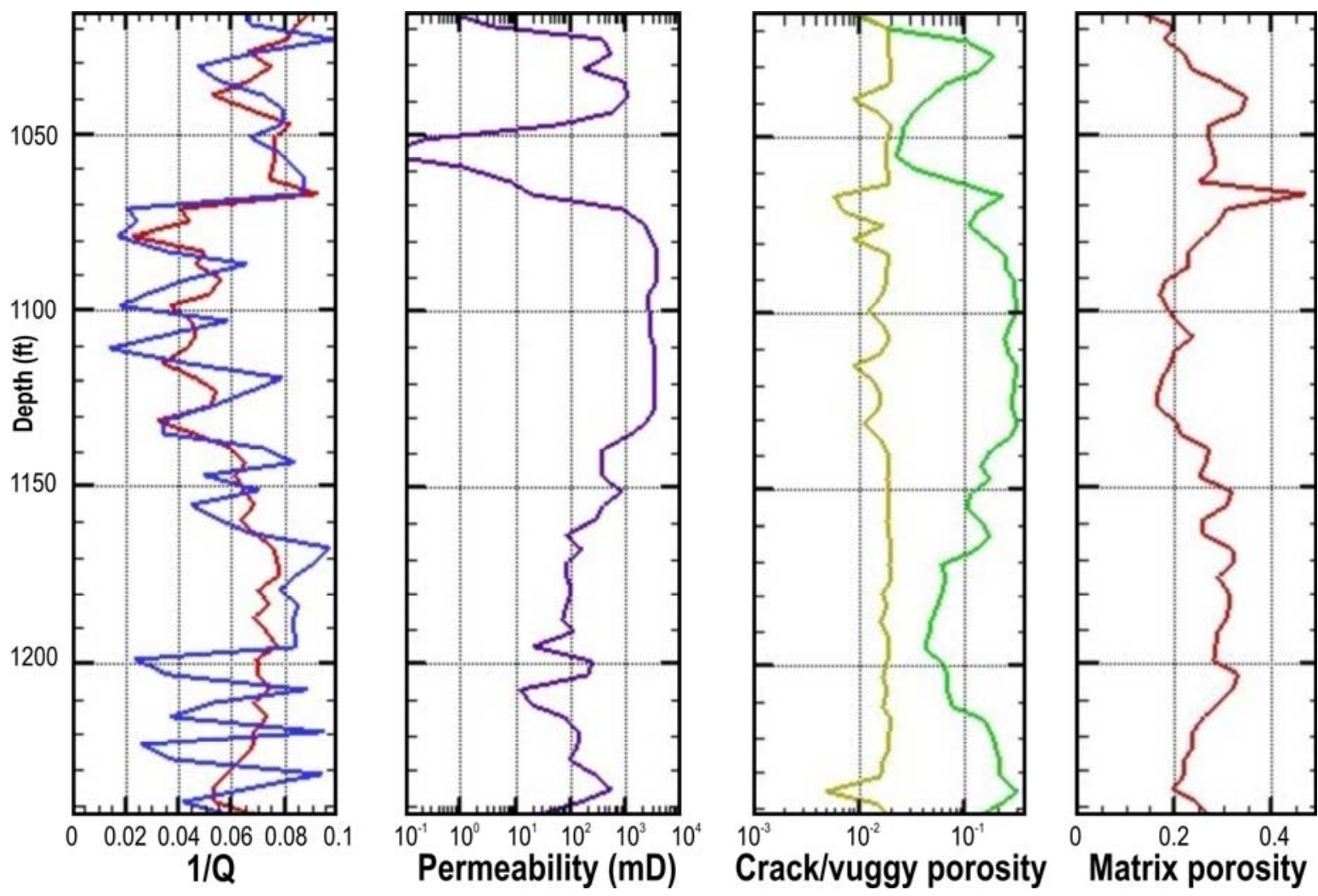

Figure 34. Comparison between observed (gray line) and predicted (black line) attenuation logs with the permeability, vuggy porosity, and matrix porosity logs. The porosities are given in decimal fraction. 
These secondary and primary porosity logs were obtained from the simultaneous inversion of the resistivity and velocity logs recorded in well PBF10 (Kazatchenko et al., 2004). The $1 / Q$ plot shows that, on average, the pore structure of the formation indeed affects acoustic wave attenuation. Predicted attenuation in terms of primary and secondary porosity was calculated with a regression equation given in Parra et al. (2004). In this interpretation the attenuation was well predicted by the three porosity types. The plots illustrate that permeability indirectly affects attenuation correlation in the carbonate units. This is observed in the strong correlation between permeability and the vuggy porosity log. In fact, the permeability trends are reflected in the vuggy porosity log.

To further explain attenuation data we use a double porosity model based on the mesoscopic flow in a poroelastic medium, which indicates that pore fluid in different porosity types responds with different changes in fluid pressure (Pride and Berryman, 2003a). This model requires the primary and secondary porosities as well as the matrix and vuggy bulk modulus. In the next section we present an approach to calculate these parameters from well logs and core data as a means to predict the synthetic attenuation in a double porosity system.

\section{B. Rock Formation Properties and Modeling}

Since we have the vuggy and matrix porosity logs, we can calculate the matrix bulk modulus, $K_{m}$ as a function of depth using the equation

$$
K_{m}\left(\phi_{m}\right)=\rho_{b}\left(V_{p}^{2}-\frac{4}{3} V_{s}^{2}\right),
$$

where $\rho_{b}=\left(1-\phi_{m}\right) \rho_{s}+\phi_{m} \rho_{f}$,

$$
V_{p}=5079.3-4990 \phi_{m} \text {, }
$$

and $\quad V_{s}=2481.1-2330 \phi_{m}$.

where $\phi_{m}$ is the matrix porosity in decimal fraction.

Equations (21c) and (21d) were predicted from fully saturated core measurements (see Kazatchenko et al., 2004 and Parra et al., 2003). In these equations, Vp and Vs are the P-wave and S-wave velocities measured at the core scale. In a similar manner we predict the bulk modulus based on well logs. In this case the P-wave and S-wave velocity equations are obtained from the cross plots between the velocities and the total porosity $\phi_{t}$ in percent, as shown in Figure 35. 

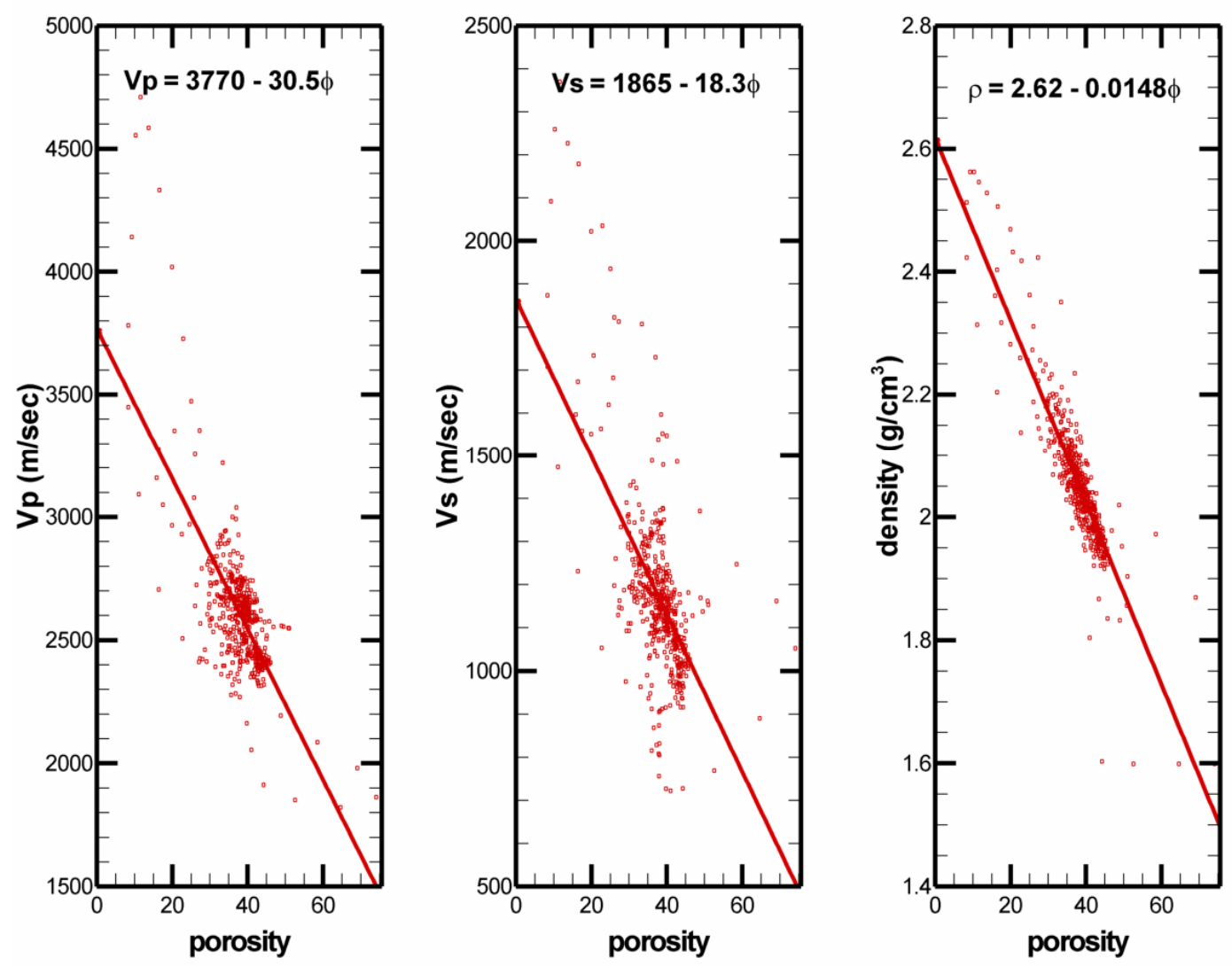

Figure 35. Crossplots of P-wave and S-wave velocity logs and density log, with the total porosity log in percent.

The equations are given by

$$
\begin{gathered}
V_{p}^{l}=3770-30 \phi_{t}, \\
V_{s}^{l}=1865-18 \phi_{t}, \\
\text { and } \quad \rho_{t}=2.62-0.0148 \phi_{t} .
\end{gathered}
$$

The bulk modulus based on Equations $(22 a)-(22 c)$ is given by

$$
K\left(\phi_{t}\right)=\rho_{t}\left(V_{p}^{(l) 2}-\frac{4}{3} V_{s}^{(l) 2}\right) .
$$

This bulk modulus, based on well log data, represents the effective fully saturated bulk modulus of the porous formation. Since the double porosity model by Pride and Berryman (2003a) requires the bulk modulus under dry conditions, we use Gassman equations to obtain these properties. Thus, the dry bulk modulus for the matrix, $K_{m d r y}$, and 
the effective dry bulk modulus of the double porosity system, $K_{d r y}$, are given, respectively, by

$$
K_{m d r y}\left(\phi_{m}\right)=\frac{K_{m}\left(\phi_{m}\right)\left(\frac{\phi_{m} K_{s}}{K_{f}}+1-\phi_{m}\right)-K_{s}}{\frac{\phi_{m} K_{s}}{K_{f}}+\frac{K_{m}\left(\phi_{m}\right)}{K_{s}}-1-\phi_{m}},
$$

and

$$
K_{d r y}\left(\phi_{t}\right)=\frac{K\left(\phi_{t}\right)\left(\frac{\phi_{t} K_{s}}{K_{f}}+1-\phi_{t}\right)-K_{s}}{\frac{\phi_{t} K_{s}}{K_{f}}+\frac{K\left(\phi_{t}\right)}{K_{s}}-1-\phi_{t}},
$$

where $K_{s}$ is the bulk modulus of the solid grain, $K_{f}$ is the bulk modulus of the water, and $\phi_{t}$ is the total porosity (volume fraction) related to the matrix and vuggy porosity by the equation

$\phi_{V}+\phi_{m}\left(1-\phi_{V}\right)=\phi_{t}$.

Where $\phi_{m}, \phi_{V}$, and $\phi_{t}$ are in decimal fraction.

To determine the bulk modulus associated with the vuggy porosity, we use an expression that relates the overall drained bulk modulus of the double porosity system including the vugs, $K_{d r y}$, with the porous matrix phase with effective properties $K_{m d r y}$, $K_{s}$, and $\phi_{m}$. We can thus write the following expression (Berryman and Wang, 1995):

$$
\frac{1}{K_{d r y}\left(\phi_{t)}\right)}=\frac{1-\phi_{V}}{K_{m d r y}\left(\phi_{m}\right)}+\frac{\phi_{V}}{K_{V}} .
$$

Then the $K_{V}$ is given by

$$
K_{V}=\frac{\phi_{V} K_{m d r y}\left(\phi_{m}\right) K_{d r y}\left(\phi_{t}\right)}{K_{m d r y}\left(\phi_{m}\right)-\left(1-\phi_{V}\right) K_{d r y}\left(\phi_{t}\right)}
$$

In equation (7), $K_{V}$ is denoted as the effective vug space bulk modulus, (1- $\left.\phi_{V}\right)$ represents the volume fraction of the total volume, and the vuggy phase occupying the remaining fraction of the volume is denoted by $\phi_{V}$.

In Figure 36 the bulk modulus of the dry matrix and the vugs are given as a function of depth. For comparison, the vuggy porosity, matrix porosity, and total porosity are given in Figure 34. The curves show that the matrix bulk modulus is greater in the carbonate zone at 326-348 $\mathrm{m}(1070-1140 \mathrm{ft}$.) or at low matrix porosity, and is lower in the region between depths of 320 and $363 \mathrm{~m}$ (1050 and $1190 \mathrm{ft}$.), where the matrix porosity is about 30 percent. This makes sense, because in a porous medium when the 
primary porosity increases, the matrix bulk modulus should decrease and the matrix became weaker. In the vuggy zone the vuggy porosity is higher (about 30 percent) and the matrix porosity is less (18 percent). In general, the vug space bulk modulus is smaller than the matrix bulk modulus. Although the vug space modulus $K_{V}$ is much smaller that the matrix bulk modulus $K_{m d r y}$, both curves have similar patterns.

At this point we have the input parameters to calculate attenuation based on the double porosity model developed by Pride and Berryman (2003a). These parameters are the bulk modulus of the carbonate matrix and the vug pore space, porosities, density, and permeability. The double porosity system is represented by phase 1, the matrix, and phase 2 , the vugs. The matrix is characterized by a matrix porosity and the vugs by a vuggy porosity. We assume that the double porosity system is represented by spherical vugs embedded in a porous stiff matrix with interconnected pore structure, and the phases have volume fractions $v_{1}$ and $v_{2}$ within an averaging volume of the combined pore structure of matrix and vuggy porosities. This allows us to assume that the composite's shear modulus can be determined using the expression (Pride and Berryman, 2003a) given by

$$
G=\frac{(1-\phi) G_{s}}{(1+c \phi)}
$$

where $\phi=\phi_{V} v_{2}+\phi_{M} v_{1}$ and $\mathrm{c}$ are the consolidated parameter $2<\mathrm{c}<10$. We consider phase 2 (the vugs) to be in the form of spheres of radius $a$ embedded within a radius $\mathrm{R}$ sphere of the two-phase pore structure. For example, if we assume that each vug of radius $a$ is surrounded by a pore matrix having a fixed radius $\mathrm{R}=3 a$, then the volume fractions are $v_{2}=(a / R)^{3} \approx 0.037$ and $v_{1}=0.963$. These parameters allow the model to calculate the scale length, $L=1.98 a$, that "characterizes the average distance in the porous matrix (phase 1) over which the fluid pressure still exists in the final approach to equilibrium," Pride and Berryman (2003a). Additional physical parameters are the solid grain modulus $K_{s}=72.2 \mathrm{GPa}$, shear grain modulus $G_{s}=38.6 \mathrm{GPa}$, grain density $\rho_{s}=2.65 \mathrm{~g} / \mathrm{cm}^{3}$, fluid viscosity $\eta=0.001 P \bullet s$, and fluid velocity $V_{f}=1500 \mathrm{~m} / \mathrm{s}(4920 \mathrm{ft} / \mathrm{s})$. 


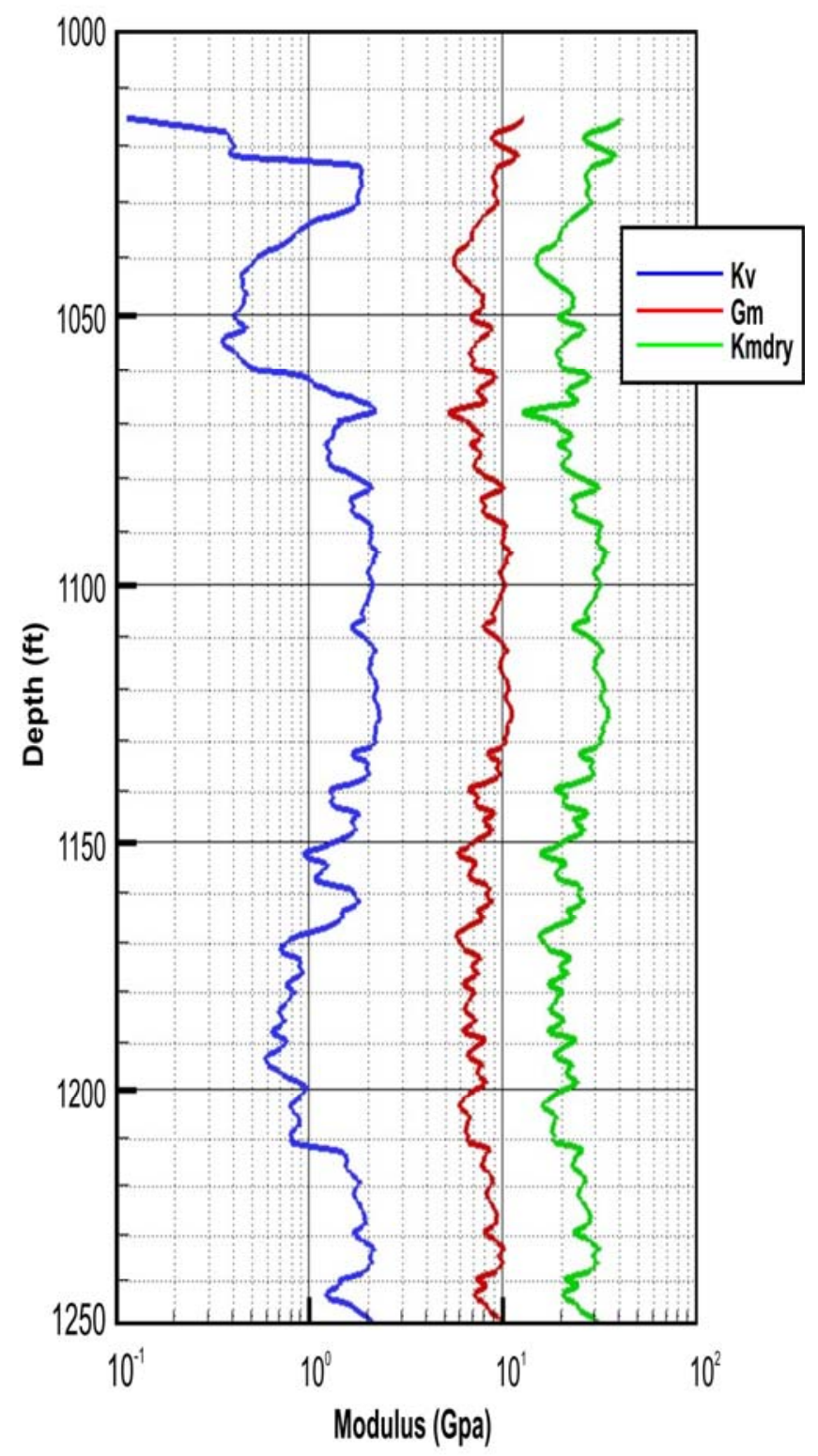

Figure 36. Comparison of matrix bulk and shear modulus with the vug space bulk modulus.

Before we calculate the attenuation $\log$ as a function of depth at the source center frequency of $10 \mathrm{kHz}$, we investigate frequency dependent attenuation for different vug radii and permeabilities. In the vuggy carbonate zone, vug size varies in the pore matrix, with typical lengths from 1-8 cm (0.03-0.26 ft). Based on NMR logs (Parra et al., 2001), we find that permeability in the region of interest varies from 1-4 Darcies. First we select the well log parameters for the highly vuggy and permeable carbonate zone at a depth of $335 \mathrm{~m}$ (1100 ft.), next we vary the scale length, $L$, from about $2-8 \mathrm{~cm}$, and then we select the less permeable zone (chalky carbonate) and vary the scale length for it as well. Figure 37 shows attenuation curves for the vuggy carbonate zone at $335 \mathrm{~m}(1100 \mathrm{ft}$.). 


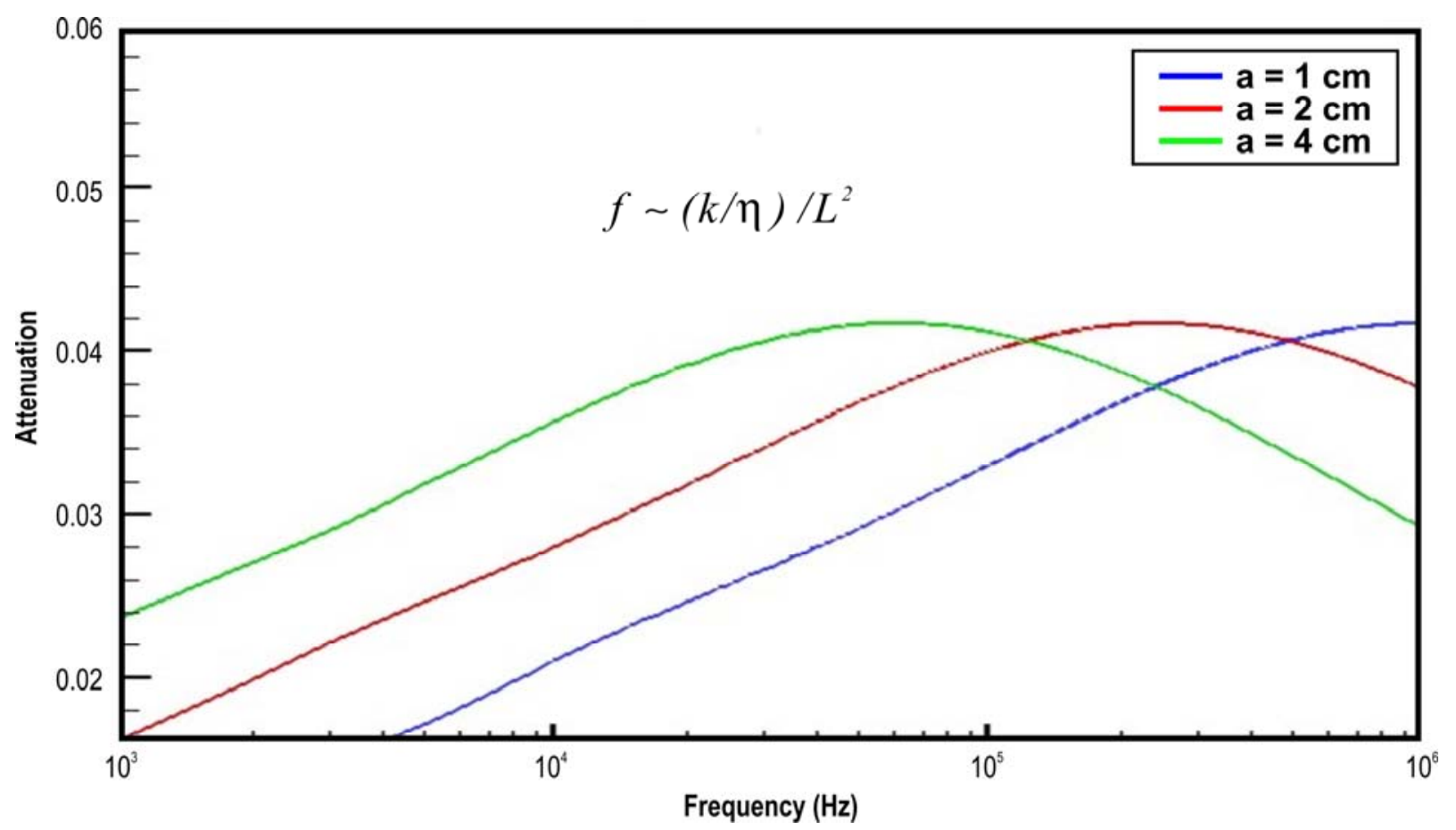

Figure 37. Attenuation curves illustrating the length scale effects on the attenuation associated with the vuggy carbonate at a depth of $335 \mathrm{~m}$ (1100 ft.). The frequency is inversely proportional to the characteristic length, $L$. This makes the attenuation peaks shift downward with increasing vug size.

In Figure 37, the attenuation peaks shift toward the high frequencies as vug size decreases. This is based on the expression given by

$$
f \approx(k / \eta) / L^{2},
$$

where $k$ is the permeability. Since we are expecting an average attenuation of 0.038 at 10 $\mathrm{kHz}$, the vugs should have a characteristic length $L=8 \mathrm{~cm}(0.26 \mathrm{ft}$.)in a permeable medium of near 3 Darcies. In a similar way we calculate attenuation curves for the chalky carbonate zone at $357 \mathrm{~m}$ (1170 ft.); in this case the vug porosity is low, but the matrix porosity is high, so the contrast between both moduli is very high. This produces a higher attenuation of about 0.085 associated with a characteristic length of $2 \mathrm{~cm}(0.066 \mathrm{ft}$.) and a permeability of $100 \mathrm{mD}$ (see Figure 38). The attenuation peaks are in the lower frequency region because they have been shifted to the lower permeability. 


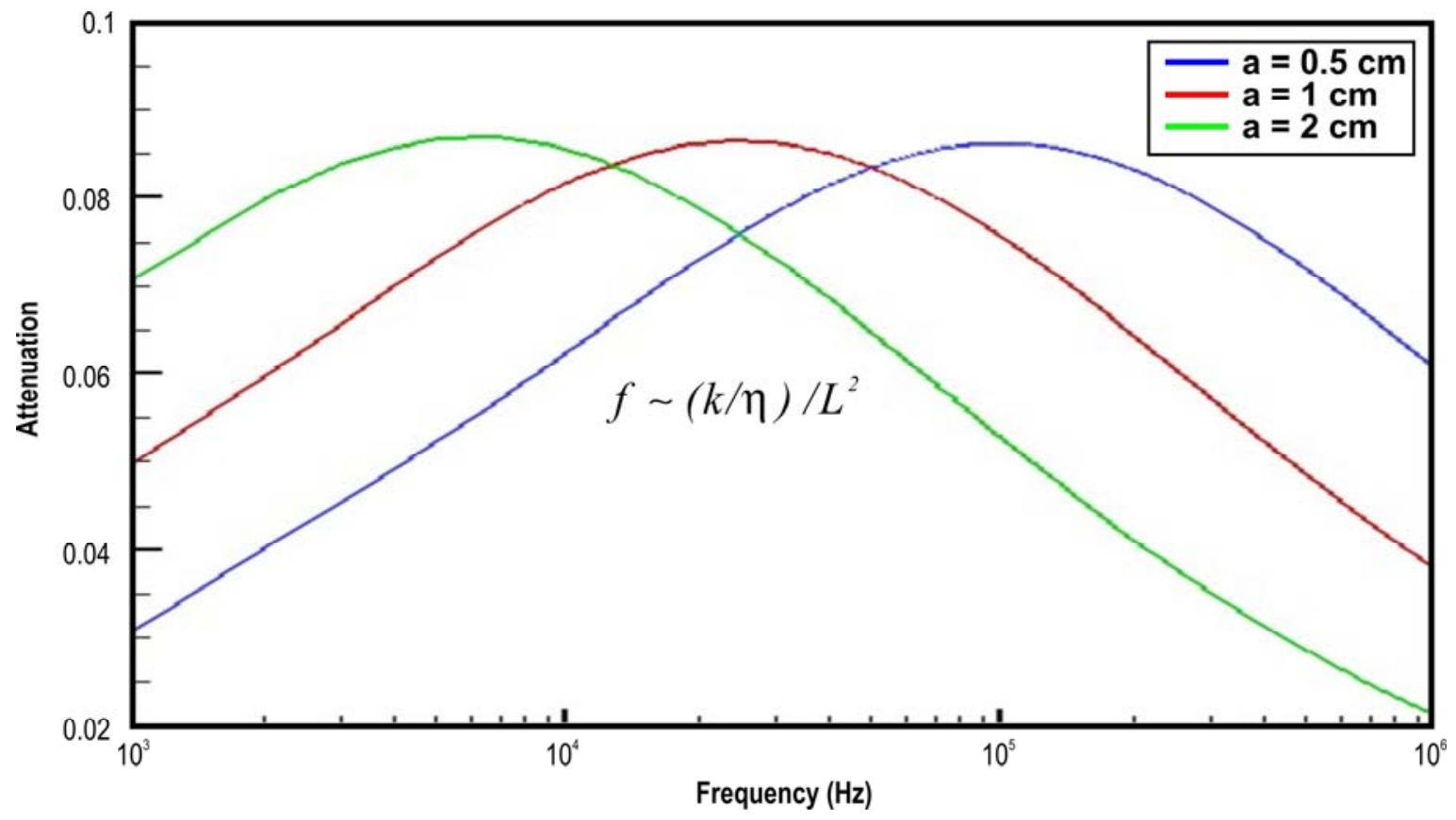

Figure 38. Attenuation curves illustrating the length scale effects on the attenuation associated with the chalky carbonate at a depth of $357 \mathrm{~m}$ (1170 ft.). The frequency is inversely proportional to the characteristic length, $L$. This makes the attenuation peaks shift downward with increasing vug size.

The final step is to calculate the attenuation $\log$ at $10 \mathrm{kHz}$ as a function of depth. Figure 39 shows the model-based attenuation (heavy line) with the observed estimated attenuation curve (gray line). In general, the agreement between both logs is very good. There are two zones with higher attenuation and a third zone of lower attenuation. The figure shows a comparison of the two attenuation logs with the lithological column to aid in visualizing regions of low and high attenuations. The vuggy carbonate is characterized by a $Q$ that varies from 25 to 50 based on the estimated attenuation and for an average $Q$ of 30 based on the synthetic log. This occurs because the acoustic attenuation based on $P$ head waves having an average wavelength of $30 \mathrm{~cm}$ (1 ft.) is sensitive to small inhomogeneities, and the synthetic log is computed with effective average parameters. In the zone above the vuggy carbonate unit, the estimated attenuation is corrected for the sandstone layer to produce a smoother signature that is due to the intrinsic attenuation (Parra et al., 2004). On the other hand, in the synthetic log, the effect of the sandstone layer is not removed. As a result, in this region the synthetic log presents more variability than the estimated attenuation.

In the region below the vuggy carbonate, the estimated and calculated attenuation agree well, except below $366 \mathrm{~m}$ (1200 ft.) where the sonic log data captures the effects of small inhomogeneities that cannot be predicted with the double porosity model. In the last track of Figure 39, we plot the assumed value of the vug radius as a function of depth that best fit the observed and calculated attenuation logs. This $a$-log follows the trend of the vuggy porosity log and its values are consistent with those observed in cores (Parra et al., 2003). 
The heterogeneous conditions of the zone below $357 \mathrm{~m}$ (1170 ft.) occur when the carbonate formation has collapsed, forming a porous chalky carbonate zone that becomes muddy and soft, which is characterized by a lossy heterogeneous structure of $Q=12.5$. On the other hand, the stiff pore structure in the highly permeable carbonate between depths of 323-357 m (1060-1170 ft.) allows water flowing from the porous matrix to the interconnected vugs, resulting in a higher $Q$ that varies from 25 to 50 .
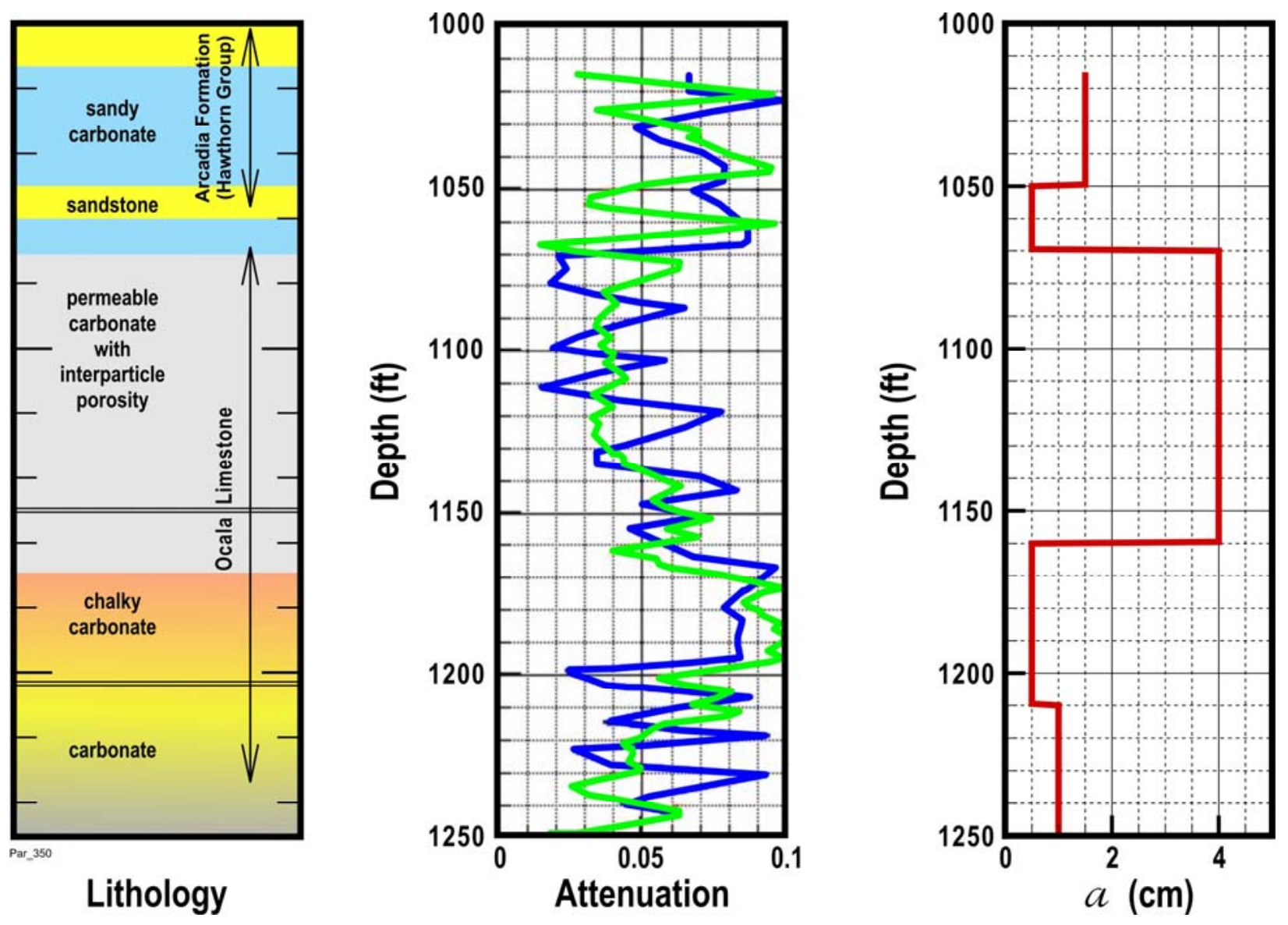

Figure 39. Comparison between observed (gray line) and calculated (heavy line) attenuation logs with the lithology at the south Florida aquifer. The a-log shows the change of vug size in the lithological column.

\section{Conclusions}

The double porosity model is consistent with the experimental attenuation measurements, and the model results explain the physics of the measured attenuation. The suitability of the double porosity model to interpret the attenuation $\log$ is supported by the strong correlation between the attenuation and the vuggy and matrix porosity logs. The results suggest that $Q$ logs can discriminate between lithology and can provide information on the pore structure when integrated with cores and other logs. In this study 
the model results show that, in permeable formations, pore structure can be related to fluid flow between the pore matrix and the vugs. In particular, permeability indirectly influences attenuation through the statistical correlation of attenuation and pore structure. This is evident from the strong correlation between permeability and interconnected vuggy porosity. Permeability trends are reflected in the vuggy porosity logs. Attributes of the chalky carbonate layer reveal that this highly porous rock is characterized by low bulk moduli and low density. Based on knowledge of the primary and secondary porosities, we are able to calculate the matrix and space vuggy moduli and use them as input parameters for the poroelastic modeling. The model allows us to predict attenuation at the sonic log peak frequency of $10 \mathrm{kHz}$ and at a broadband frequency range. The results also suggest that the pores in the vuggy carbonate are stiffer than those in the chalky carbonate; as a result, attenuation is lower in the vuggy carbonate. 


\section{PERMEABILITY AND POROSITY IMAGES BASED ON P-WAVE SURFACE SEISMIC DATA}

Surface and borehole geophysical techniques have been used to delineate aquifers and estimate the fluid and rock physical properties of the subsurface. In particular, surface methods such as seismic, resistivity, electromagnetic (EM), and ground penetrating radar (GPR) have been widely used. Sandersen and Jorgensen (2003) characterized buried valleys in western Denmark and their inferred influence on groundwater resources and vulnerability using transient electromagnetic (TEM), seismic, and gravimetric surveys. Day-Lewis et al. (2003) used borehole geophysics to investigate specific water and rock properties. They applied crosswell difference-attenuation GPR to monitor saline-tracer migration in an experiment at the U.S. Geological Survey Fractured Rock Hydrology Research Site in Grafton County, New Hampshire. Paine (2003) integrated borehole EM measurements with surface EM soundings to determine salinization depth and to relate ground conductivity to chloride content. Sumanovac and Weisser (2001) evaluated seismic and resistivity geophysical methods for hydrogeological mapping in karst terrains. They found that the high-resolution seismic method allowed them to map fracture zones and identify lithological boundaries associated with a contact between dolomites and compact limestone, and that the resistivity method provided information on water circulation. Cardimona et al. (1998) compared images of seismic reflections and ground penetrating radar in a shallow aquifer. The results showed that seismic data imaged clay lenses, whereas low-frequency radar profiles did not provide clear results. Yamamoto (2003) introduced a new inversion method of acoustic velocity and attenuation to extract permeability from acoustic data based on the Biot theory of poroelasticity. He used this method to extract permeability from attenuation tomography data recorded in a shallow limestone aquifer in south Florida.

High-resolution crosswell technology for petrophysical analysis, in conjunction with core and geophysical log data, was used by Parra et al. (2003a) to relate lateral impedance variations in a south Florida carbonate aquifer to permeability and porosity variations. High-resolution crosswell seismic provided good information on the flow units of the proposed Aquifer Storage and Recovery (ASR) horizon at three scales (core, borehole, and crosswell seismic) between test wells PBF10 and PBF13 at a separation of 330 feet $(100 \mathrm{~m})$. The high-resolution crosswell data was obtained with the use of a piezoceramic X-series source; the source sweep length was 1.2 seconds at a sampling rate of $200-2000 \mathrm{~Hz}$. The peak frequency was about $1500 \mathrm{~Hz}$, which provided a resolution of $1.4 \mathrm{~m}(4.5 \mathrm{ft})$. However, since deep-water wells in south Florida are drilled at separations exceeding $1600 \mathrm{~m}$ (one mile), crosswell seismic technology is often not practical, in particular for the delineation of regional flow units related to large-scale ASR implementation.

In this section, we evaluate the applicability of surface reflection seismic as a possible technique for characterizing the subsurface hydrogeology at the Florida site. For this purpose, we ran a 2D seismic line parallel to the line containing test wells PBF10 and PBF13, and we used impedance images based on reflection data and well logs for imaging flow unit properties. One test well had a complete suite of logs, and the other well had a few logs, a crosswell seismic study, and a 2D P-wave seismic data set. In 
addition, in the case of this aquifer, it was desirable to minimize the drilling and construction cost by using non-intrusive sensing techniques for characterizing the subsurface geology. Since we have all the petrophysical information from one well, including permeability, one of our goals is to relate the P-wave reflection data with permeability and porosity at the borehole location so that we can produce images of these parameters. A practical way is to convert 2D P-wave reflection data to impedance, and then correlate the derived impedance log at the borehole scale with the permeability and porosity logs to produce permeability and porosity profiles. We describe work undertaken to evaluate the applicability of P-wave (compressional) surface seismic reflection data for imaging both high permeability flow units and low permeability confining units over a wide range of depths in a regional carbonate environment. The ultimate objective is to obtain site information over a large lateral extent without drilling costly near-spaced wells and to use the seismic data for locating additional ASR wells at the site. Important factors related to ASR implementation are lateral continuity in upper and lower confining units and permeable zones (ASR Horizon) that allow high water volume flows to occur. The depth range of interest is generally from ground surface to $610 \mathrm{~m}$ (2000 ft.). We demonstrate that previous crosswell images based on highresolution crosswell seismic data and well logs can be used to verify how well the $2 \mathrm{D}$ surface seismic reflection method captures important flow units within a proposed ASR horizon in the Floridan aquifer. The 2D surface seismic reflection profile is further evaluated with synthetic model data by including well logs and attenuation data. The well log data, reflection seismic and 2D surface reflection seismic data were recorded in feet; however, all the plots were converted to SI units.

\section{A. Seismic Reflection Method}

\section{Acquisition and processing}

A 2D seismic P-wave line survey was conducted at the proposed ASR site in southern Palm Beach County, Florida, adjacent to Hillsboro Canal and Water Conservation Area 1 shown in Figure 40(a). The surface seismic reflection test profile was acquired along the north side of the Hillsboro Canal. The seismic line is parallel to the line containing the vertical test boreholes PBF10 and PBF13, which are $100 \mathrm{~m} \mathrm{(330}$ $\mathrm{ft}$.) apart. The seismic line was located at $9.1 \mathrm{~m}(30 \mathrm{ft}$.) from the plane containing the boreholes. A sketch of the site is illustrated in Figure 40(b). The P-wave profile was collected along an unpaved levee road running parallel to the canal, to which the South Florida Water Management District provided access. The topography of the test area was relatively flat except for a small drop in elevation near the beginning of the west to east transect due to construction of a water control structure (Bennett and Linton, 2002). A total of $1060 \mathrm{~m}(3,475 \mathrm{ft}$.) of reflection P-wave surface seismic profile was acquired. Reflections containing frequencies of $300 \mathrm{~Hz}$ were recovered, which was the maximum frequency input by the seismic source. 


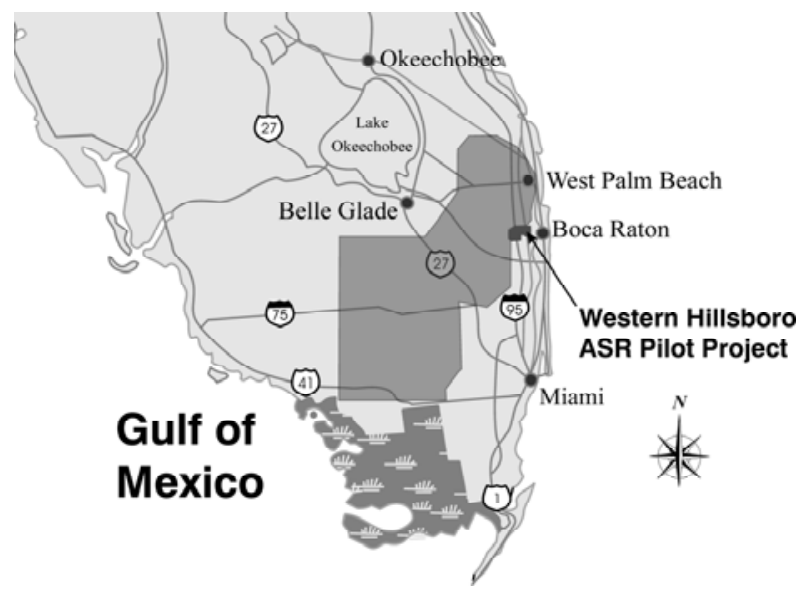

(a)

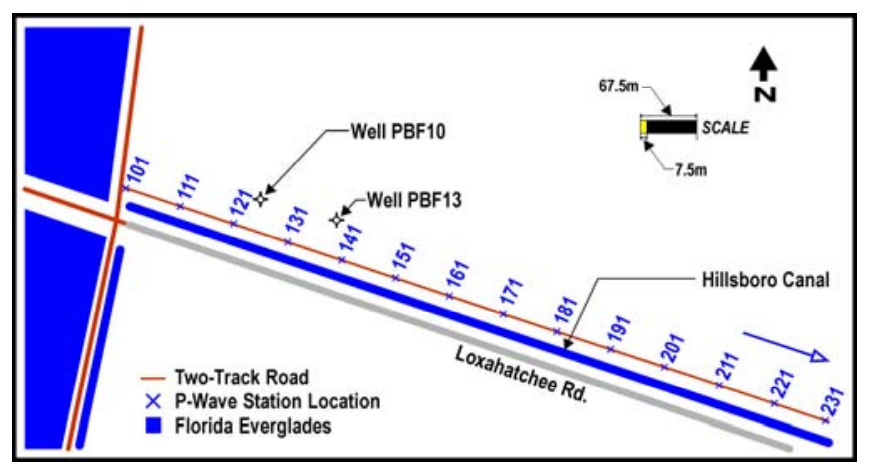

(b)

Figure 40. (a) ASR Pilot Project regional map; (b) location of seismic experiments at the western Hillsboro Aquifer Storage Recovery (ASR) site near Boca Raton, Florida. One Pwave profile of $1060 \mathrm{~m}$ (3475 ft.) was acquired at $9.1 \mathrm{~m}$ (30 ft.) from the line containing test wells PBF10 and PBF13. At the surface, the wellheads are approximately $100 \mathrm{~m}$ (330 ft.) apart. The distance between stations is $7.6 \mathrm{~m}$ (25 ft.).

The data quality is demonstrated in the raw data shown in Figure 41, an example of a shot gather recorded at station 179.5. In this case, the source was placed between stations 179 and 180. While reflections are prominent, the shot gather also includes the effects of ground roll and head waves. In the survey, the station receiver interval was 7.6 $\mathrm{m}$ (25 ft.), as was the source interval. The sweep was linear, with sweep frequencies between 28 and $300 \mathrm{~Hz}$ and a sweep length of 6 seconds. The important acquisition parameters are given in Table 7. 


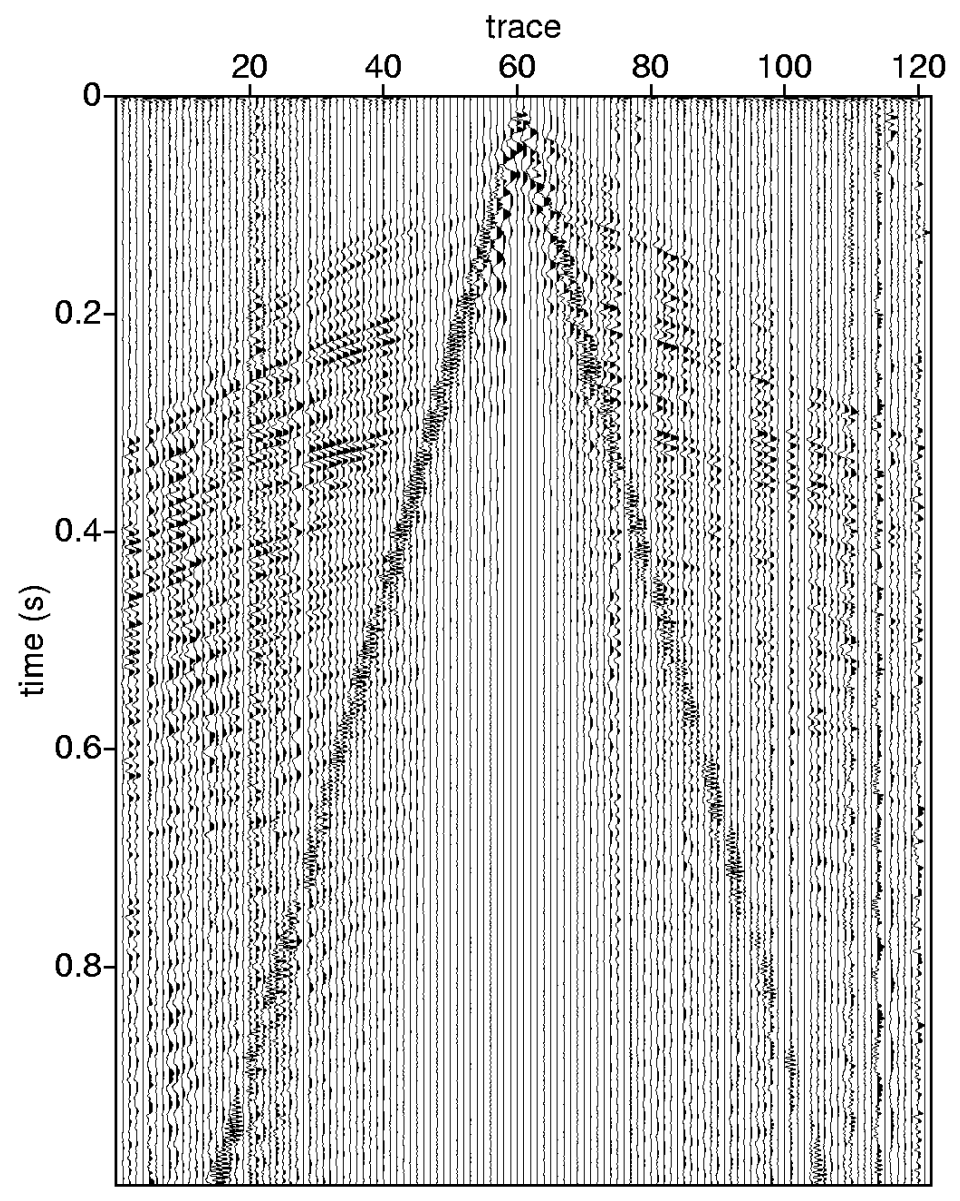

Figure 41. Shot gather recorded at station 179.5. 
Table 7. Acquisition Parameters

\begin{tabular}{|l|l|}
\hline Recording system & OYO DAS-1 \\
Source & Mini-Vibrator of T1 5000 LB peak force \\
Source weight & $300 \mathrm{lb}$ \\
Source frequency & $18-550 \mathrm{~Hz}$ \\
Geophones & $20-300 \mathrm{~Hz}$ \\
Geophones/station & 6 Geophones in-line array \\
Shot interval & $7.6 \mathrm{~m}(25 \mathrm{feet})$ \\
Receiver interval & $7.6 \mathrm{~m}(25 \mathrm{feet})$ \\
Channels & 120 \\
Sampling interval & 0.5 millisecond \\
Record length & 3 seconds \\
Pre-A/D filters & 24 bit sigma-delta A/D converter \\
Data redundancy & 60 \\
& \\
\hline
\end{tabular}

The seismic reflection profile is shown in Figure 42 as a Common Depth Point (CDP) stacked section. In this figure, red indicates maximum negative signal and blue represents maximum positive signal. The maximum vertical resolution of the P-wave data is approximately 4.6-6.1 m (15-20 ft.). A number of continuous reflectors are observed through the section, especially between 0.1 and 0.5 seconds (61-430 m or 200-1740 ft.). Events at and below 0.5 seconds [approximately $518 \mathrm{~m}$ (1700 ft.)] show an anticlinal structure between stations 135 and 200. Below 0.7 seconds [about $823 \mathrm{~m} \mathrm{(2700} \mathrm{ft.)]} \mathrm{the}$ reflections become discontinuous and are harder to follow. If we assume that the stratification beneath $823 \mathrm{~m}$ (2700 ft.) is continuous, the implication is that the maximum penetration of the P-wave data in this interval is about 0.7 seconds. The processing sequence is similar to that described in Yilmaz (2000). The initial data processing included trace editing, true amplitude gain recovery, surface consistent amplitude analysis, and compensation surface consistent deconvolution. The standard processing applied to the data included normal move-out corrections, mute analysis, surface consistent automatic static, and velocity analysis. Time-variant amplitude scaling and CDP consistent trim static were applied to the data as well. In addition, the processing flow included a time-varying gain to compensate for attenuation losses. After the data was processed, finite-difference time migration was applied. The impedance calculation was done in conjunction with well logs, so seismic impedance was calibrated against well $\log$ impedance. The concept of impedance is explained in the next section. 


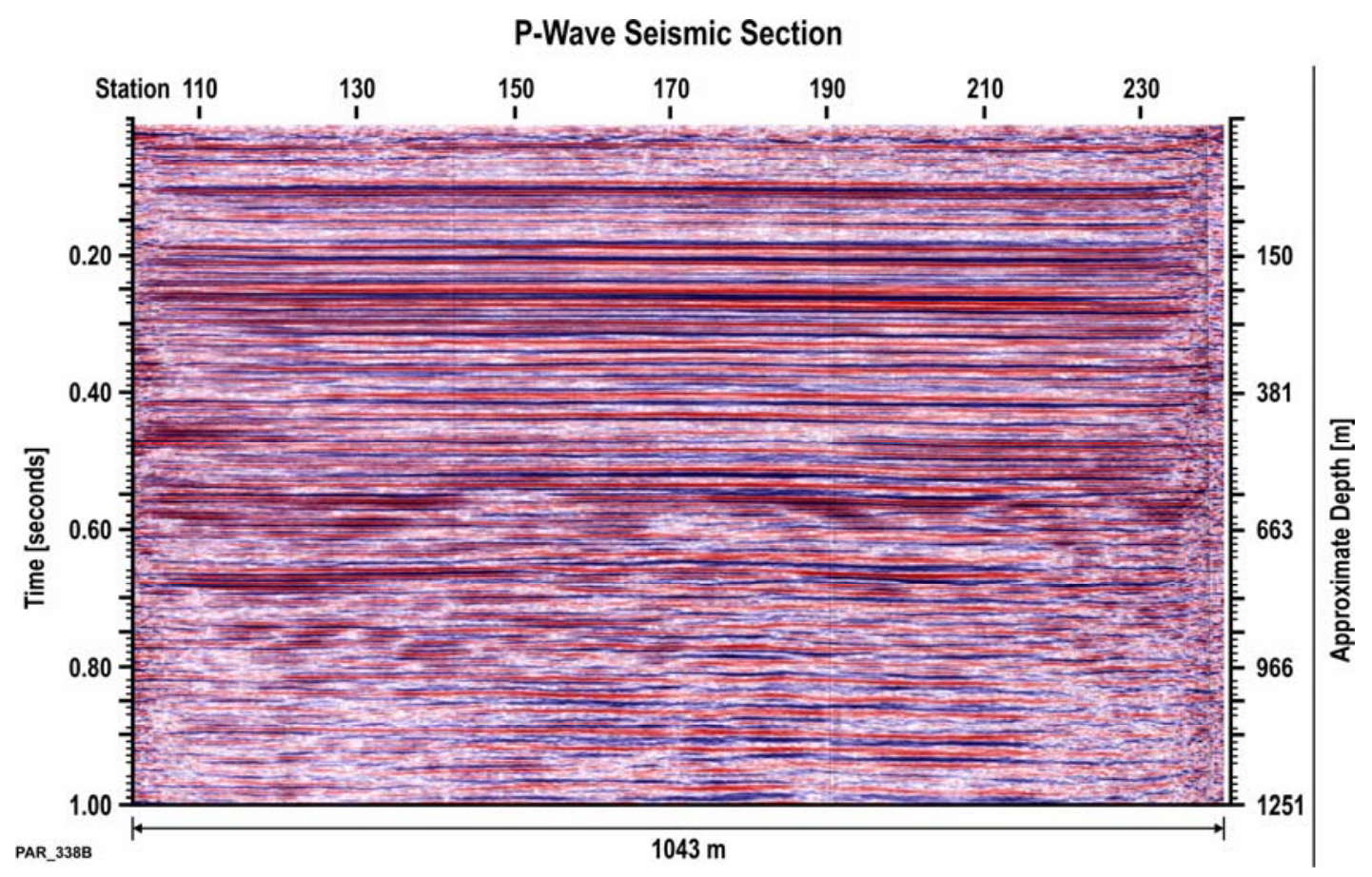

Figure 42. Observed surface seismic reflection data illustrated as a stacked CDP section.

\section{Seismic impedance and inversion algorithm}

Seismic impedance is the ratio of stress to wave velocity and is given by the product $\rho V$, where $\rho$ is the density and $V$ is the wave velocity (Aki and Richards, 1980; and Mavko et. al, 1998). A normally incident plane wave traveling from the surface of the earth and reflected at boundaries characterized by the impedance well log produces the reflectivity wave response. When the reflection coefficient associated with the reflectivity wave response is positive, the incident wave is propagating from a low impedance medium into a higher impedance medium. Alternatively, when a wave travels from a medium of higher impedance to a medium of lower impedance, the reflection coefficient is negative. Based on this concept, several researchers in the last 50 years (e.g., Peterson et al., 1955) have developed analog and computer models to produce synthetic seismograms based on well log data. Wuenschel (1960) introduced the time domain approach and Bouchon (1976) implemented controlled sources. Others have implemented modeling methods accounting for heterogeneities using finite-difference schemes (e.g., Zahradnik et al. 1994), anisotropy (Collino and Tsogka, 2001), viscoelasticity (Carcione, 1993), and poroelasticity (Hackert and Parra, 2000). The finitedifference method allows the interpreter to include additional rock physics and fluid properties in the model, thus resulting in more realistic synthetic data that more closely resemble the seismic response of actual rock formations.

The seismic data was inverted for impedance using the band-limited inversion technique given by Oldenburg et al. (1983). Briefly, the conversion from seismic reflection to impedance images requires the description of the input seismic data, $x(t)$ as the convolution of the seismic wavelet, $w(t)$, with a reflection coefficient series, $r(t)$, 


$$
x(t)=w(t) * r(t),
$$

where $t$ is time. The wavelet analysis consists of determining a filter $v(t)$ that best matches the well log reflection coefficients to the input seismic data $x(t)$ at the well log location. Thus, if the reflection coefficients from the well logs (P-wave velocity and density) are $r_{k}$, then we write the time series as

$$
\mathrm{r}(\mathrm{t})=\sum_{\mathrm{k}=1}^{\mathrm{N}} \mathrm{r}_{\mathrm{k}} \delta\left(\mathrm{t}-\mathrm{t}_{\mathrm{k}}\right) .
$$

In Equation (30), $t_{k}$ corresponds to the two-way travel time to the $k^{\text {th }}$ layer; $N$ is the total number of layers, and $r_{k}$ is the reflectivity coefficient at the interface between the $k^{\text {th }}$ and the $(k+1)^{\text {th }}$ layer; and $\delta$ is the Dirac function.

Since we know $r(t)$ from the well logs and $x(t)$ from the seismic data, we can predict the optimum wavelet $\mathrm{w}(t)$ at the borehole location using Equation (29). Then the inverse filter is determined such that

$$
w(t) * v(t)=\delta(t)
$$

For any other trace in the seismogram, $x(t)$, we convolve the trace with the filter $v(t)$. In general, if Equation (29) represents any trace in the seismogram and $w(t)$ is the known wavelet constrained by the well log, then the reflection series associated with that input trace is given by the convolution of Equation (29) and the filter $v(t)$. That is,

$$
r(t)=x(t) * v(t)
$$

The new $r(t)$ series based on the new trace $x(t)$ combined with Equation (30) gives the coefficients $r_{k}$. The acoustic impedance is related to the reflection coefficients by

$$
\mathrm{z}_{k+1}=z_{1} \prod_{j=1}^{k}\left(\frac{1+r_{j}}{1-r_{j}}\right)
$$

where $z_{l}$ is the surface impedance. The impedance inversion is designed to best satisfy Equation (33) while values are constrained to match the well-log impedance in the vicinity of the well. Away from the well location, the impedance is constrained by lateral continuity, but it may vary as the seismic reflections vary in amplitude or depth.

In summary, a filter is determined based on well logs and seismic data at the borehole location. This filter is subsequently applied to all the traces in the seismogram. This operation gives the reflection time series associated with each trace. The impedance is recovered in terms of the reflection coefficients at each trace location. Since part of the operation is done in the frequency domain and the data is band-limited, the more advanced procedure developed by Oldenburg et al. (1983) is required to recover the acoustic impedance from reflection seismograms.

\section{Computer model}

To evaluate the observed seismic data we use a modeling approach based on the solution of a plane wave propagating in an arbitrary plane in a viscoelastic multilayered medium (Parra and Hackert, 2001). The solution assumes that the $\mathrm{z}$ plane (vertical axis) is the axis of symmetry and the layer interfaces are paralleled to the horizontal $\mathrm{x}$-y plane. The differential wave equation governs the wave motion in each layer. The displacement and stress fields satisfy the boundary conditions of continuity of displacements and 
tractions across each interface as well as the radiation condition at infinity. To avoid precession problems caused by the growing exponential in individual matrices for large wave numbers, we used the global method to calculate the vector wave displacement in the frequency domain (Schmidt and Tango, 1986). That is, the plane-wave matrix solution operates in the frequency domain, and the final spectrum is transformed to the time domain by means of Fourier transformation. This modeling scheme provides realistic synthetic seismograms that can represent the surface seismic reflection data.

\section{B. Petrophysics}

Well logs and cores from an exploratory test well identified as PBF-10 (see Figure 1) were used to create a lithological column between depths of $229 \mathrm{~m}$ (750 ft.) and $381 \mathrm{~m}$ (1250 ft.). The permeability log is derived from the Nuclear Magnetic Resonance (NMR) $\log$ (Chang et al., 2001). P-wave and S-wave velocity and $Q$ logs are obtained from the full waveform sonic data. These logs are given in Figure 43 together with the density and porosity logs and the lithological column. Based on the relation between the velocity tomography image, the P-wave velocity log (Parra et al., 2003a), and the permeability log, three flow units were identified that have been related to the velocity. These permeability anomalies - beginning at depths of $296 \mathrm{~m}$ (970 ft.), $311 \mathrm{~m}(1020 \mathrm{ft}$.),

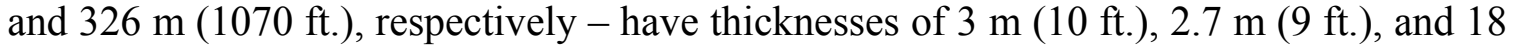
$\mathrm{m}(60 \mathrm{ft}$.), and they are associated with changes in impedance at the same depths. The first flow unit has a low P-wave velocity and $28 \%$ porosity. This unit correlates with a high gamma ray anomaly due to $15-20 \%$ of phosphatic sands. The second flow unit has a high velocity and an average porosity of $20 \%$ and is associated with a low gamma ray anomaly. Based on core data, this unit contains separated vugs that do not contribute to overall permeability; the permeability is controlled by the matrix of the rock formation. The last flow unit is a low velocity region within the Ocala Limestone that corresponds to a clean carbonate, which is confirmed by the low natural gamma-ray anomaly. The porosity in this region varies between $28-43 \%$.

The parameter $Q$ is the inverse of the wave attenuation $\left(Q^{-1}\right)$ and was

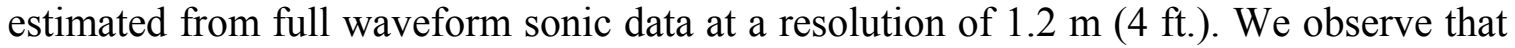
the $Q \log$ generally correlates with the lithology and captures the texture of the sand and limestone units (Parra et al., 2004). When P-waves excited by a source propagate through a carbonate aquifer characterized by heterogeneities of different dimensions, such as variations in lithology and vuggy porosity, some regions respond with different fluid pressure than others. The associated pressure diffusion attenuates the wave energy (Pride and Berryman, 2003; Parra et al., 2003b). 

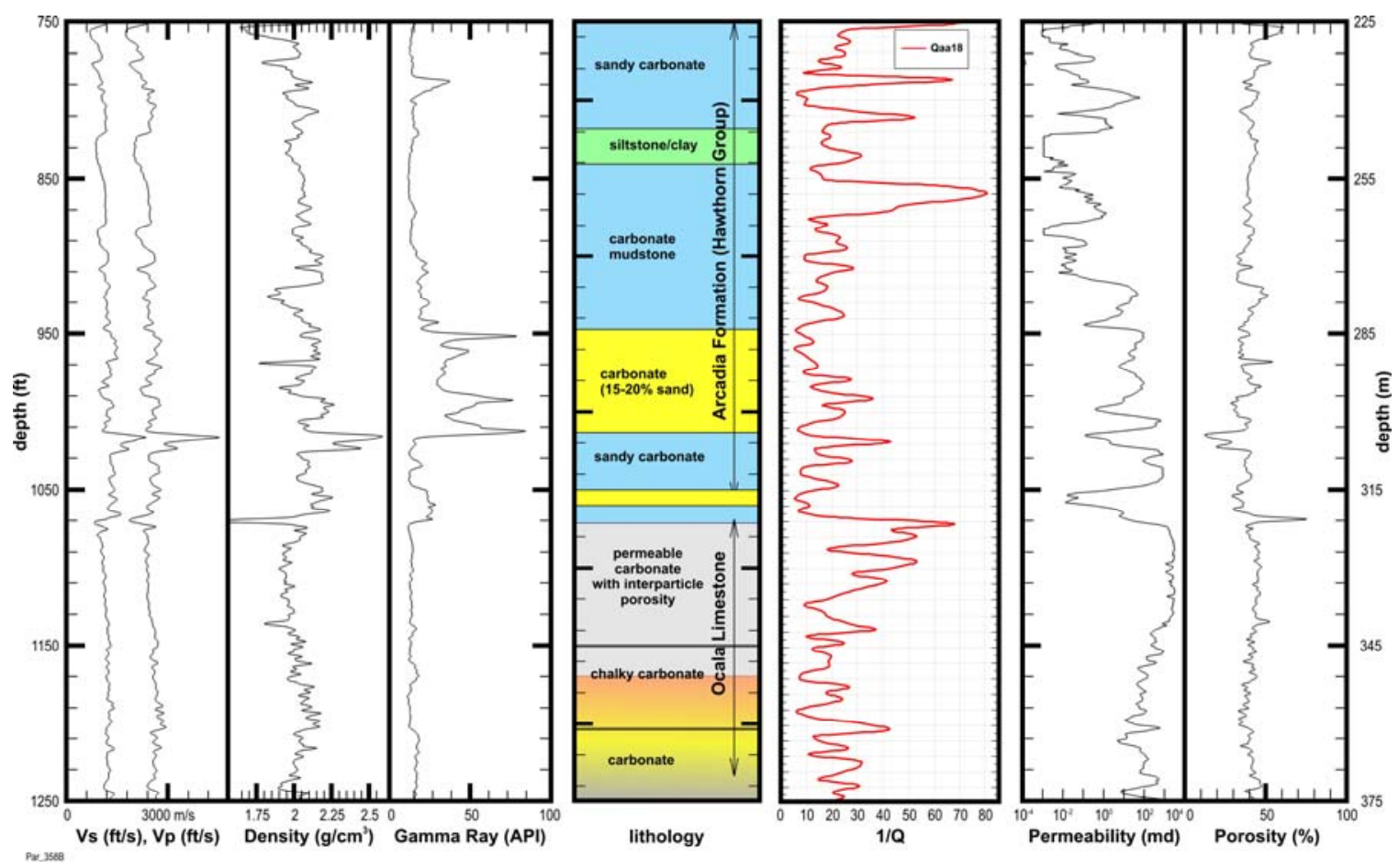

Figure 43. Well logs from well PBF10, south Florida. Comparison of Vp, Vs, density, and gamma ray log with lithology, $Q$ log, permeability and porosity.

The effect of attenuation can be observed by comparing the lithology with the full waveform sonic $\log$ between depths of $229 \mathrm{~m}$ (750 ft.) and $381 \mathrm{~m}(1250 \mathrm{ft}$.). Figure 44 shows depth intervals where acoustic waves are strongly attenuated and how those intervals correspond to the lithological column. In this study we use $Q$ only to generate synthetic seismograms to better understand the observed seismic data.

\section{Analysis and Interpretation}

\section{Modeling}

Synthetic seismograms are useful as a quality control measure to evaluate whether the seismic reflection data have captured the reflections observed at the borehole location. In addition, the comparison between observed and synthetic data allows the interpreter to relate seismic attributes to the petrophysics.

We attempt to use the modeling approach that includes viscoelasticity in the layered earth model. This requires including the quality factor as a function of depth, as shown in Figure 4, and having the density log as well as the Vp and Vs logs. The overall effect of including the quality factor in the model is to simulate realistic waveforms that are broadened by the combined effects of scattering and intrinsic attenuation. Indeed, the perfect match is not always possible. Since we do not know the exact source function for the surface seismic reflection survey, in some places we cannot obtain the perfect match. 
We are also assuming a constant $Q$ at all frequencies. If there is a frequency dependence of $Q$ in some part of the formation, then we may expect a mismatch. Because this subject is beyond the scope of the present paper, we refer the reader to Dvorkin and Nur (1993) and to Mavko et al. (1998) for more details on the frequency dependence of $Q$. The modeling scheme previously addressed provides realistic seismograms to represent the surface seismic reflection data.

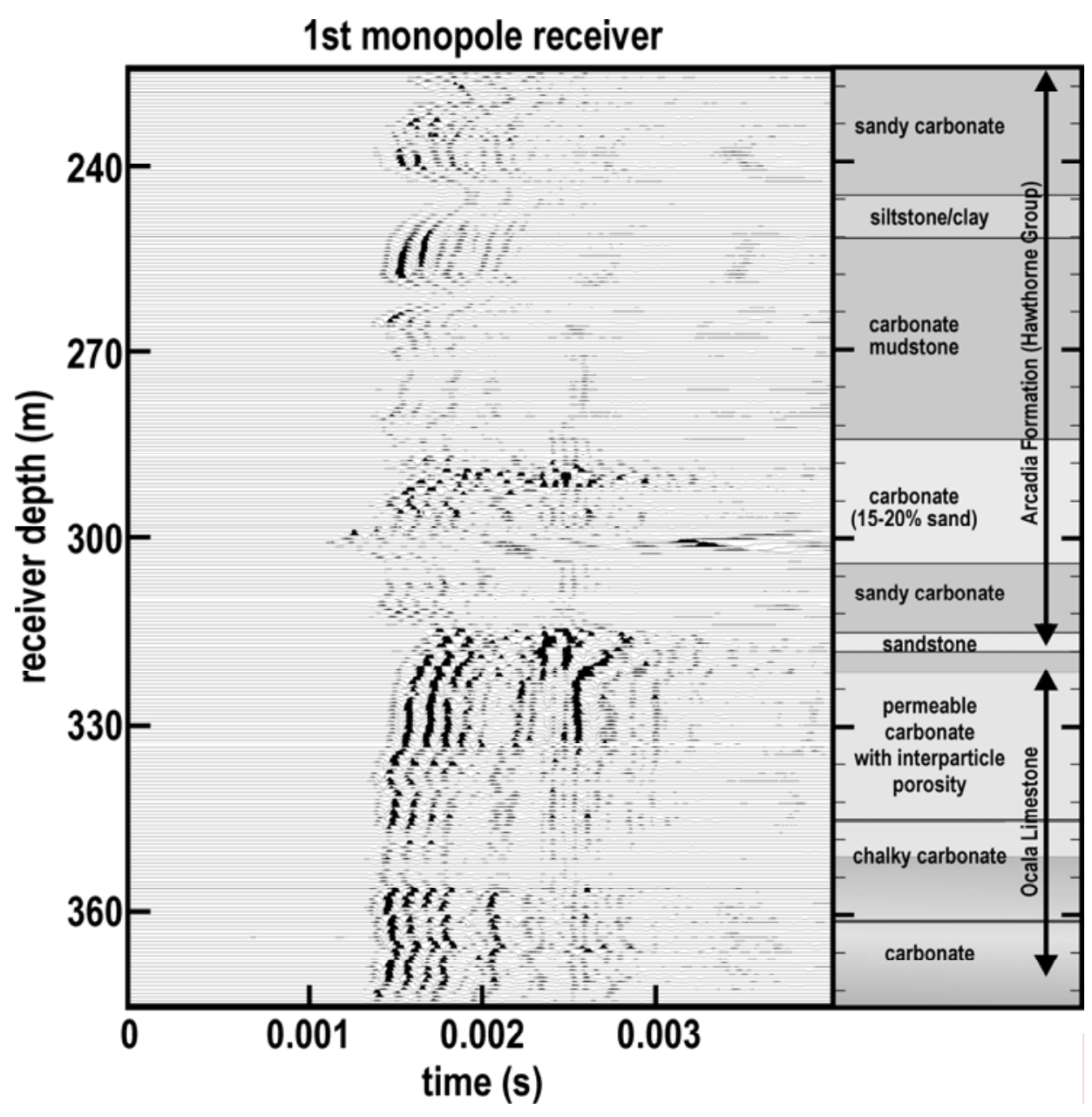

Figure 44. A comparison of full waveform monopole sonic data with the lithological column between $229 \mathrm{~m}$ (750 ft.) and $381 \mathrm{~m}$ (1250 ft.).

Thus, synthetic seismograms based on the well logs were produced and compared with the corresponding migrated seismogram (Figure 45) to assess the quality of the data and the continuity of the major lithofacies and boundaries. The plane-wave matrixmethod solution produces all reflections, including multiples, from a given layered model. We created one synthetic seismogram using the $Q \log$ given in Figure 42. The synthetic and observed seismograms matched well, so we are confident that the surface seismic reflection data at the well location are delineating the same features that appear in the well logs. 


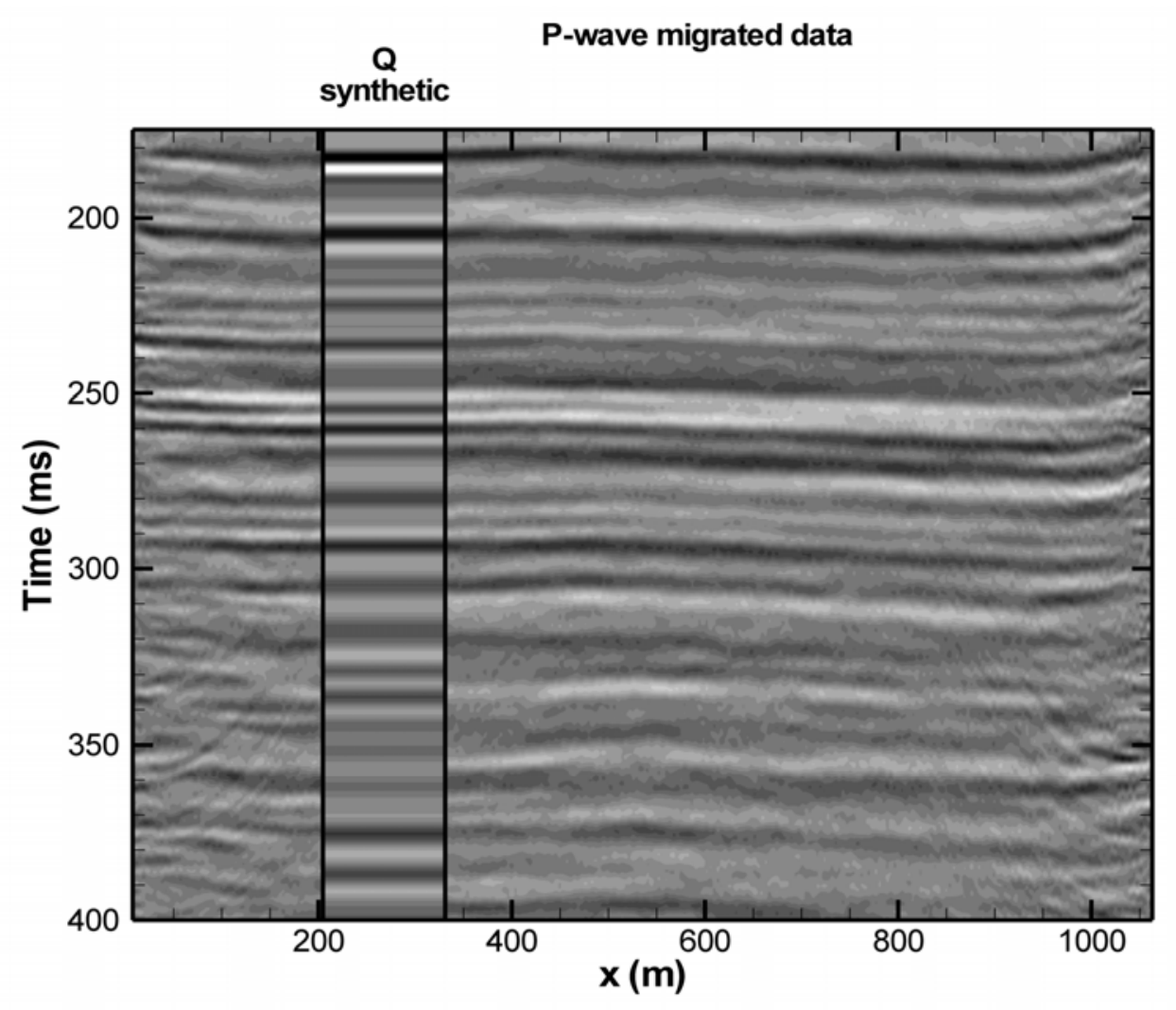

Figure 45. 2D surface seismic reflection data. The inset shows synthetic seismograms based on the well logs for attenuating media. There is a good match between the synthetic seismograms and the real data.

\section{Seismic inversion}

We invert the surface seismic data for impedance using the Vp and density logs and the band-limited method (a feature of the STRATA software, developed by the Hampson and Russell Company). The inversion helps us estimate the acoustic impedance from band-limited normal incidence reflection seismograms. The method consists of constructing an impedance model that satisfies the processed seismogram constrained by well logs. The impedance model, based on geological and geophysical information, forms a well-constrained estimation of the true earth impedance. We constrain the impedance model using P-wave velocity and density logs, and we perform a controlled inversion using linear programming. In general, the inversion is done trace by trace, and its required preprocessing includes data checks and possible corrections.

The surface P-wave reflection data from South Florida was inverted to produce an impedance image using this band-limited inversion algorithm. The resulting impedance image shows the geologic structure of the site more clearly than the reflection data alone (Figure 46). With the $3.8 \mathrm{~m}$ (12.5 ft.) spacing of the CDP, the image covers 


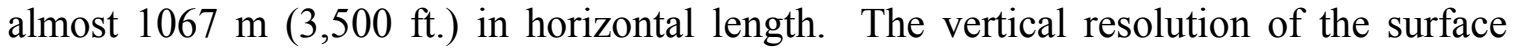
seismic data [wavelength equal to $9.1 \mathrm{~m} \mathrm{(30} \mathrm{ft.)]} \mathrm{is} \mathrm{much} \mathrm{less} \mathrm{than} \mathrm{that} \mathrm{of} \mathrm{the} \mathrm{sonic} \mathrm{well}$ $\operatorname{logs}$ [wavelength of $0.3 \mathrm{~m}(1 \mathrm{ft}$.)], as previously noted. In Figure 47 we show a comparison between the impedance well log and the surface seismic derived-impedance to illustrate the seismic resolution versus sonic well log resolution.

Figure 48 shows an expanded view of the P-wave impedance from the seismic line in the region where it overlaps the crosswell reflection data. This region covers the crosswell seismic measurements between wells PBF10 and PBF13. The figure also shows a comparison between borehole log impedance parameters (P-wave velocity and density) and the impedance computed from the surface seismic section. The impedance image of Figure 48 is compared with the P-wave impedance derived from the crosswell reflection data given in Figure 49. This comparison allows us to visualize the resolution of the surface seismic versus the crosswell seismic. The crosswell data have roughly three times the bandwidth of the surface seismic data, with a corresponding increase in vertical resolution. This is the reason for the sharp features observed in the crosswell impedance image that resemble the scale of the sonic log much better than that of the $2 \mathrm{D}$ seismic reflections. The crosswell data also provide slightly better horizontal resolution, but of course are limited to the zone between the two test wells.

The above comparison between the 2D seismic and crosswell images supports the use of surface seismic reflection data for imaging the major geological features associated with flow units. For example, the boundaries between the sandstone and the sandy carbonate at $305 \mathrm{~m} \mathrm{(1000} \mathrm{ft.)} \mathrm{and} \mathrm{between} \mathrm{the} \mathrm{sandstone} \mathrm{and} \mathrm{the} \mathrm{vuggy} \mathrm{carbonate}$ at $326 \mathrm{~m}$ (1070 ft.) are well defined by 2D seismic. Below this last boundary, a low impedance region is observed in the image. This low impedance slowly increases to reach a boundary at a depth of about $366 \mathrm{~m}$ (1200 ft.). To analyze how the impedance changes are related to the hydrological characteristics of the aquifer, we construct cross plots of the impedance at the borehole location versus the permeability and porosity well logs. 


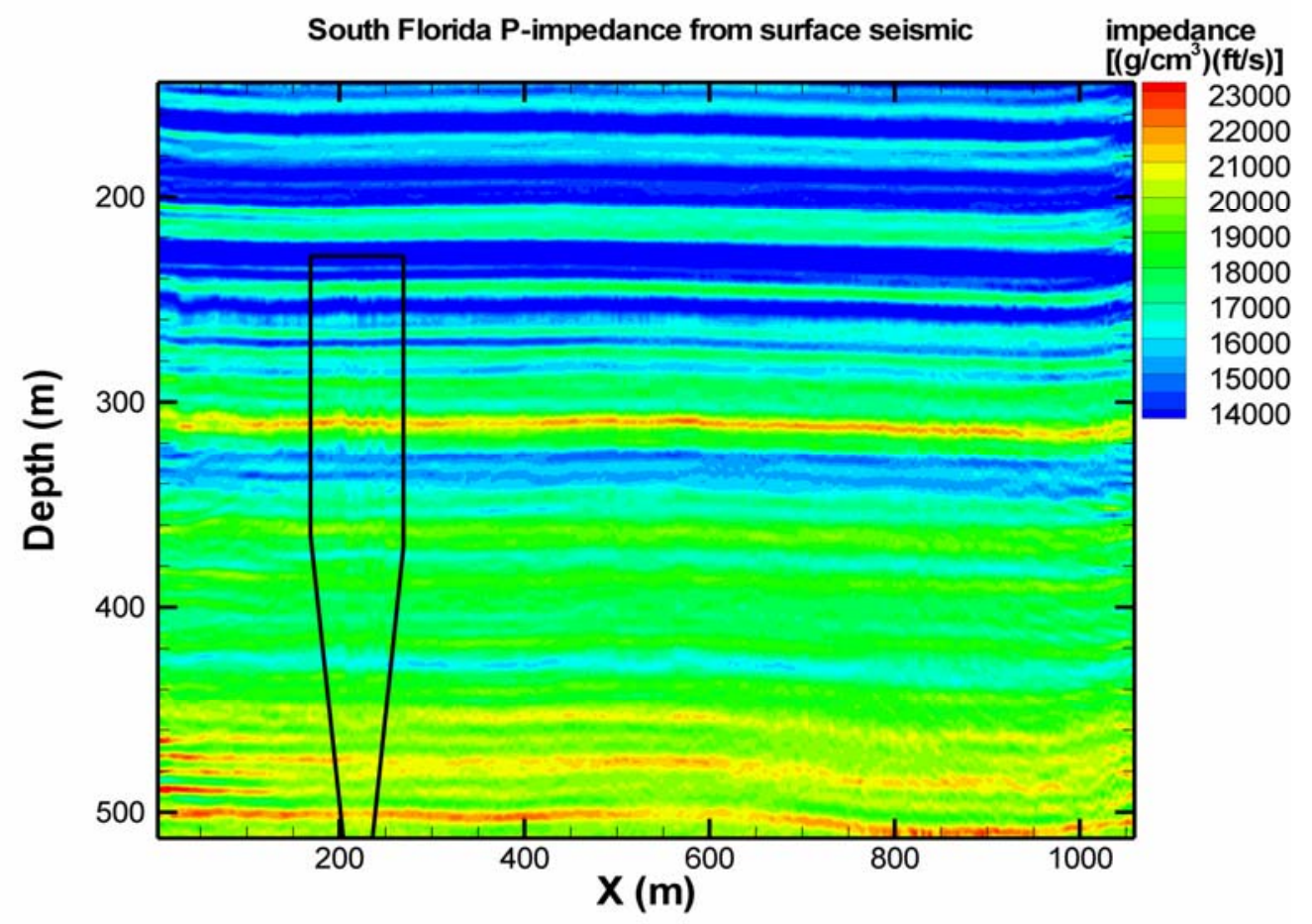

Figure 46. P-wave impedance from south Florida 2D surface seismic data. The heavy line indicates the approximate data coverage of a recent crosswell reflection survey.

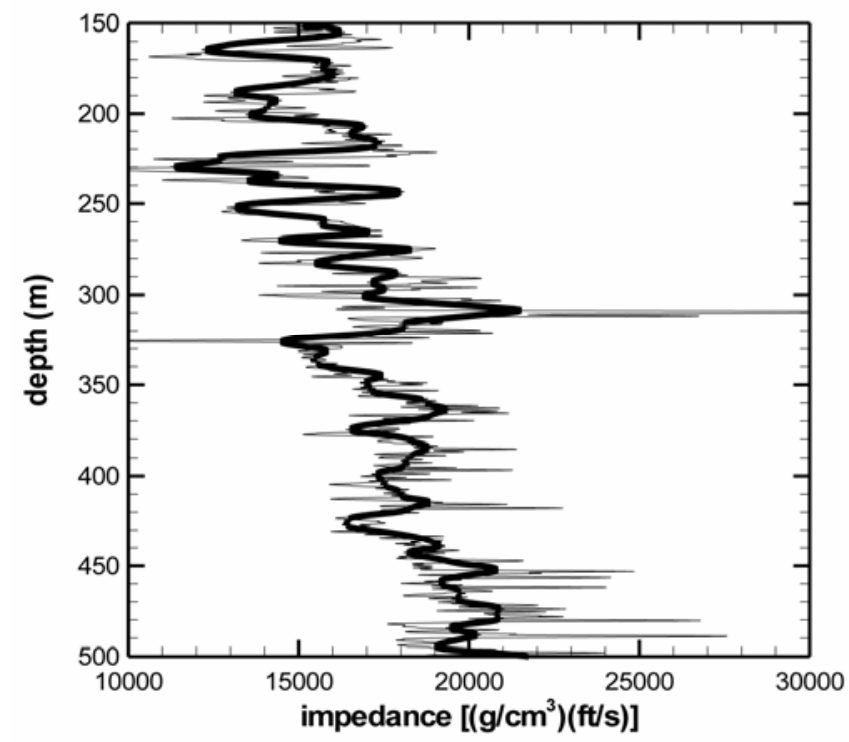

Figure 47. Comparison of well log impedance (black) from the PBF10 well and impedance from the surface seismic line at the same location (red). The well log impedance is used to constrain the seismic impedance. 


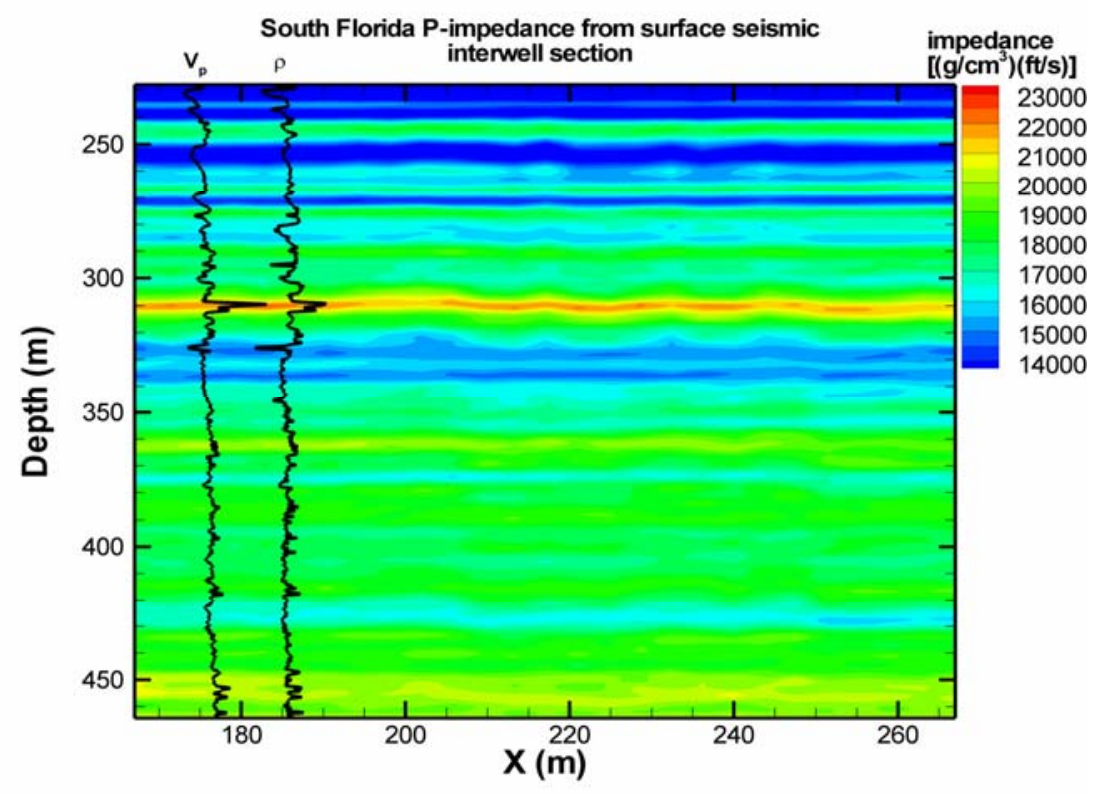

Figure 48. A section of the P-impedance from surface seismic data, which overlaps with the crosswell reflection data. Although the well where the logs were obtained is located at $x=167 \mathrm{~m}$ (548 ft.), the P-wave and density logs are superimposed at offsets to provide better visual comparison to the surface seismic impedance.

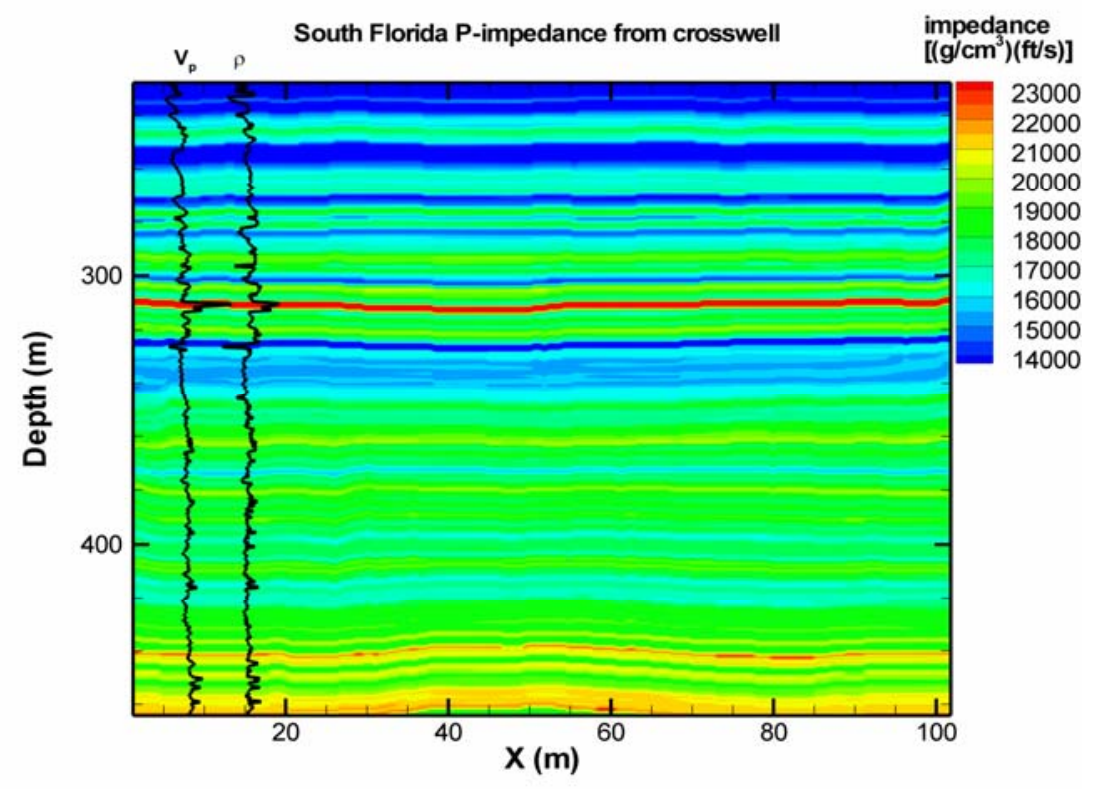

Figure 49. P-wave impedance computed from crosswell reflection data. Although the well where the logs were obtained is located at $x=0 \mathrm{~m}$, the P-wave and density logs are superimposed at offsets to provide better visual comparison to the surface seismic impedance. 


\section{Crossplots and porosity/permeability images}

To visualize the flow units in more detail, we selected the region between $290 \mathrm{~m}$ (950 ft.) and $381 \mathrm{~m}$ (1250 ft.) from the impedance image of Figure 48. This zone is bounded by well PBF10, so we were able to overlay the permeability and porosity logs from that well for comparison. We first created crossplots between the impedance and the permeability and porosity well $\operatorname{logs}$ to derive regression equations at the borehole location. We used the resulting relationships to produce permeability and porosity images at the surface seismic scale. Since we know that rock physical properties vary with depth, we do not expect perfect correlations with the impedance. The effect of the heterogeneous conditions is observed in the $Q-\log$ characterized by an attenuation average $\left(Q^{-1}\right)$ of 0.071 and a standard deviation of 0.040 , with a $1.2 \mathrm{~m}(4 \mathrm{ft}$.) resolution at different depths (see Figure 43).

The well logs in the carbonate region show a strong trend of porosity and permeability varying inversely with impedance. Crossplots of impedance versus permeability and porosity (from the well logs) were produced for the depth of $290 \mathrm{~m}$ $(950 \mathrm{ft}$.) to $381 \mathrm{~m}(1250 \mathrm{ft}$.), as shown in Figure 50 . We established empirical relationships (or impedance crossplot fit equations) for permeability (k) and porosity $(\phi)$ for these depths. The permeability fit is

$$
k=7 \times 10^{10} \exp \left(-1.1 \times 10^{-3} \mathrm{Z}\right) \text {, }
$$

where $\mathrm{k}$ is millidarcies and $\mathrm{Z}$ is impedance in $(\mathrm{ft} / \mathrm{s})\left(\mathrm{g} / \mathrm{cm}^{3}\right)$. The goodness of fit is $\mathrm{R}^{2}=$ 0.51 . The porosity fit is

$$
\phi=80-0.0024 \mathrm{Z},
$$

where $\phi$ is in percent and $\mathrm{Z}$ is impedance in $(\mathrm{ft} / \mathrm{s})\left(\mathrm{g} / \mathrm{cm}^{3}\right)$. The goodness of fit is $\mathrm{R}^{2}=$ 0.55 . To demonstrate the reliability of the regression Equations (34) and (35), we use them to predict permeability and porosity as a function of depth at the borehole location and we compare the results with the corresponding well logs.
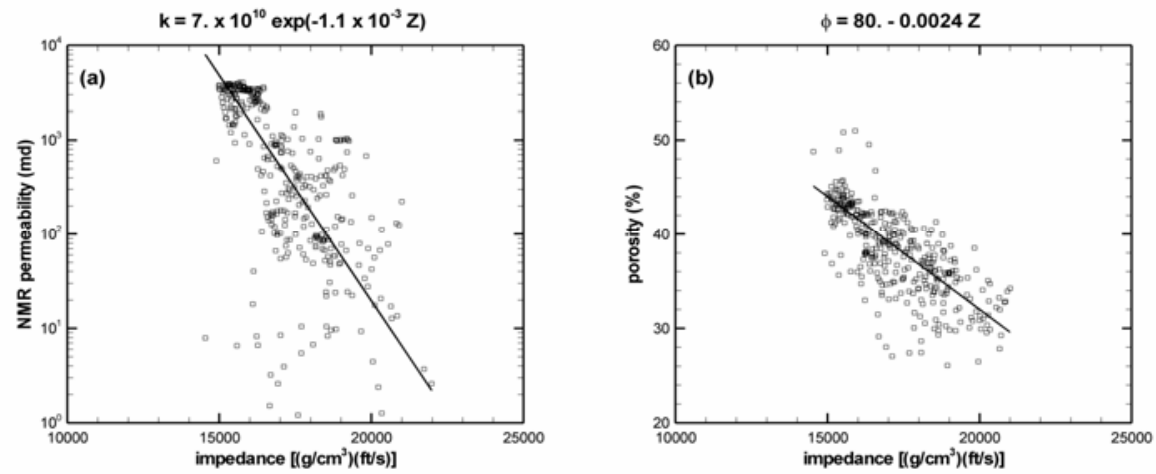

Figure 50. (a) Crossplot of well log impedance with well log NMR permeability from a 290-m (950-ft.) to a 381-m (1250-ft.) depth, and corresponding fit equation; (b) Crossplot of well log impedance with well log porosity from a 290-m (950-ft.) to a 381-m (1250-ft.) depth, and corresponding fit equation. 
This validation is performed using solely well log data. Figure 51(a) shows a comparison between the permeability well $\log$ and the permeability derived from impedance using Equation (34), and Figure 51(b) illustrates a comparison between the porosity well $\log$ and the porosity derived from impedance using Equation (35). The fit between the porosity well logs is very good. The fit between the permeability logs is very good in vuggy carbonate zones. However, in low permeability zones associated with sandstones there is a poor match between the permeability logs. In these zones (sands) we did not have enough data points to predict a relation between permeability and velocity. As a result we could not predict the permeability log very well in that region. In addition, we already know that this unit is a thin permeability barrier. Our main focus is to map the thicker vuggy carbonate zone, where the major production of water occurs. Since the model predicts the observed permeability in this interval very well, we are confident that we can use the regression equations to convert impedance images to porosity and permeability profiles in the carbonate units.

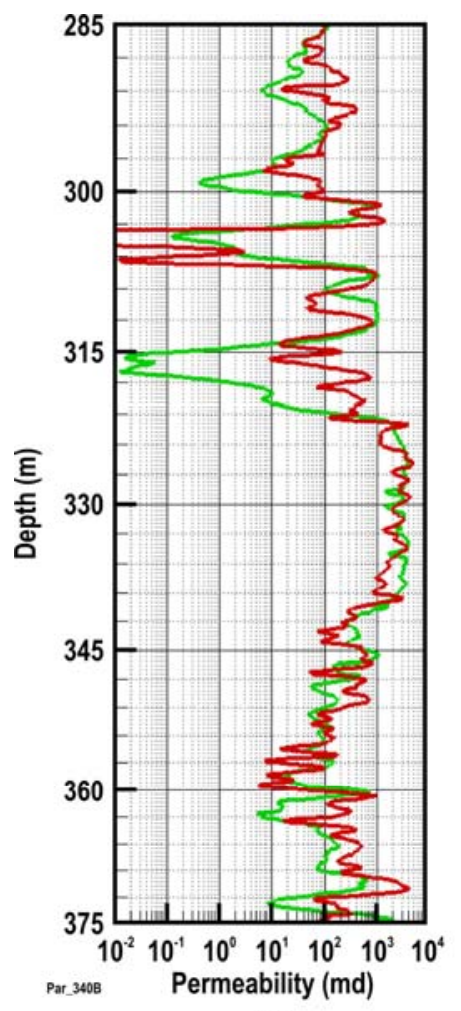

(a)

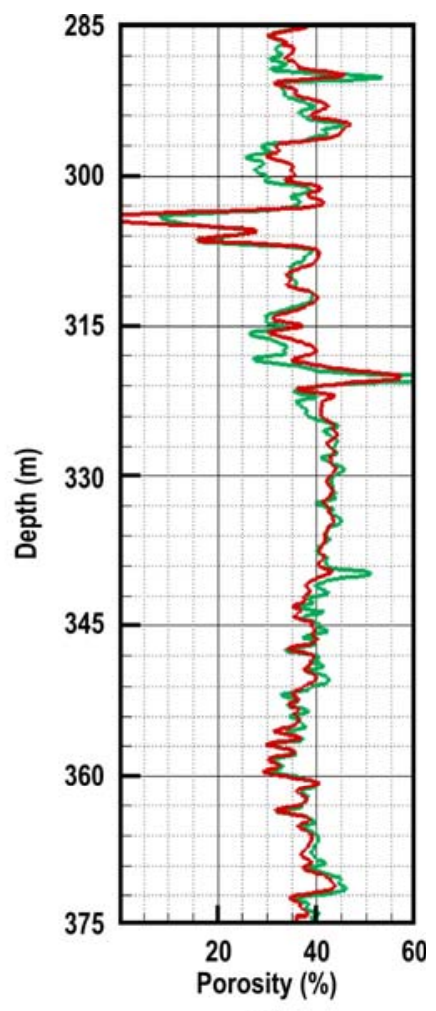

(b)

Figure 51. (a) Comparison between the NMR-permeability well log (green) and the permeability (red) derived from regression Equation (6); (b) Comparison between the porosity well log (green) and porosity (red) derived from regression Equation (7).

To convert the impedance section to permeability and porosity images (Figure 52 ), we use the regression equations for porosity versus impedance and permeability versus impedance given by Equations (34) and (35), respectively. Each pixel of the 
impedance image is converted to permeability and porosity images by replacing its value in the proper relationship given either by Equation (34) or (35). Thus, the permeability image in Figure 52 is obtained by placing the impedance values given in Figure 46 in the regression Equation (34). In a similar way the porosity image in Figure 52 is determined by inserting the impedance values given in Figure 46 into the regression Equation (35). Thus, the correlation equations used to map the impedance images into permeability and porosity images reflect trends in the lithofacies at this depth. These images contain their corresponding well logs.

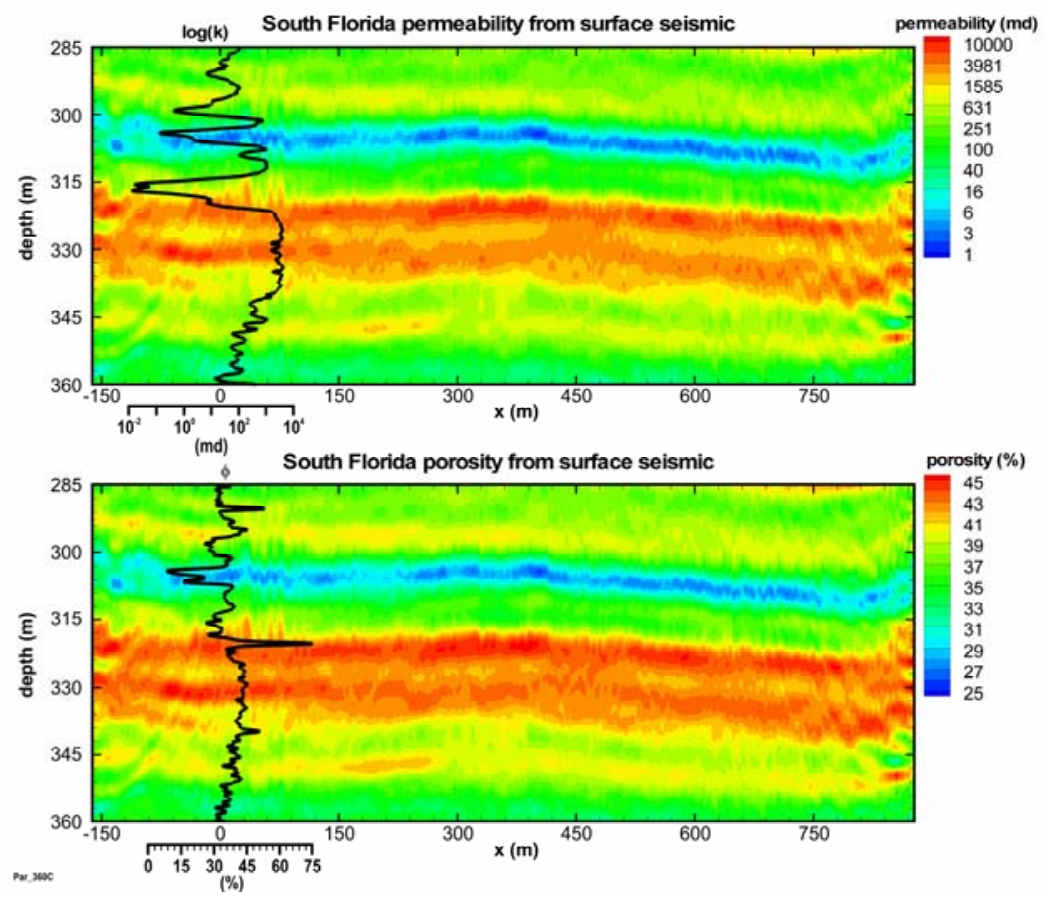

Figure 52. Permeability (top) and porosity (bottom) inferred from surface seismic $P$-impedance and correlations with well logs.

To analyze the resolution of the images we compared them with images based on crosswell seismic data, as shown in Figure 53. The crosswell images also show correlations with well logs. The images based on surface seismic data show flow units that are consistent with the well log data. For example, the red high impedance unit at

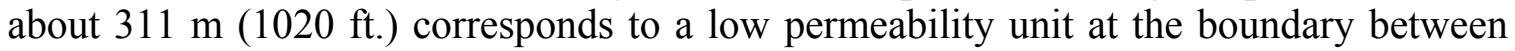
the sand and the sandy carbonate (see Figures 48 and 52). This unit is also observed in the crosswell permeability image. In the crosswell image the unit is better resolved, and it is easer to determine its thickness. The vuggy, highly permeable carbonate can also be identified in the surface seismic derived permeability and porosity images between $326 \mathrm{~m}$ $(1070 \mathrm{ft}$.) and $351 \mathrm{~m}(1150 \mathrm{ft}$.). The corresponding crosswell permeability and porosity images show much sharper images. These results suggest that the same important features captured with crosswell seismic measurements can also be visualized with 2D seismic data, although at lower resolution. 


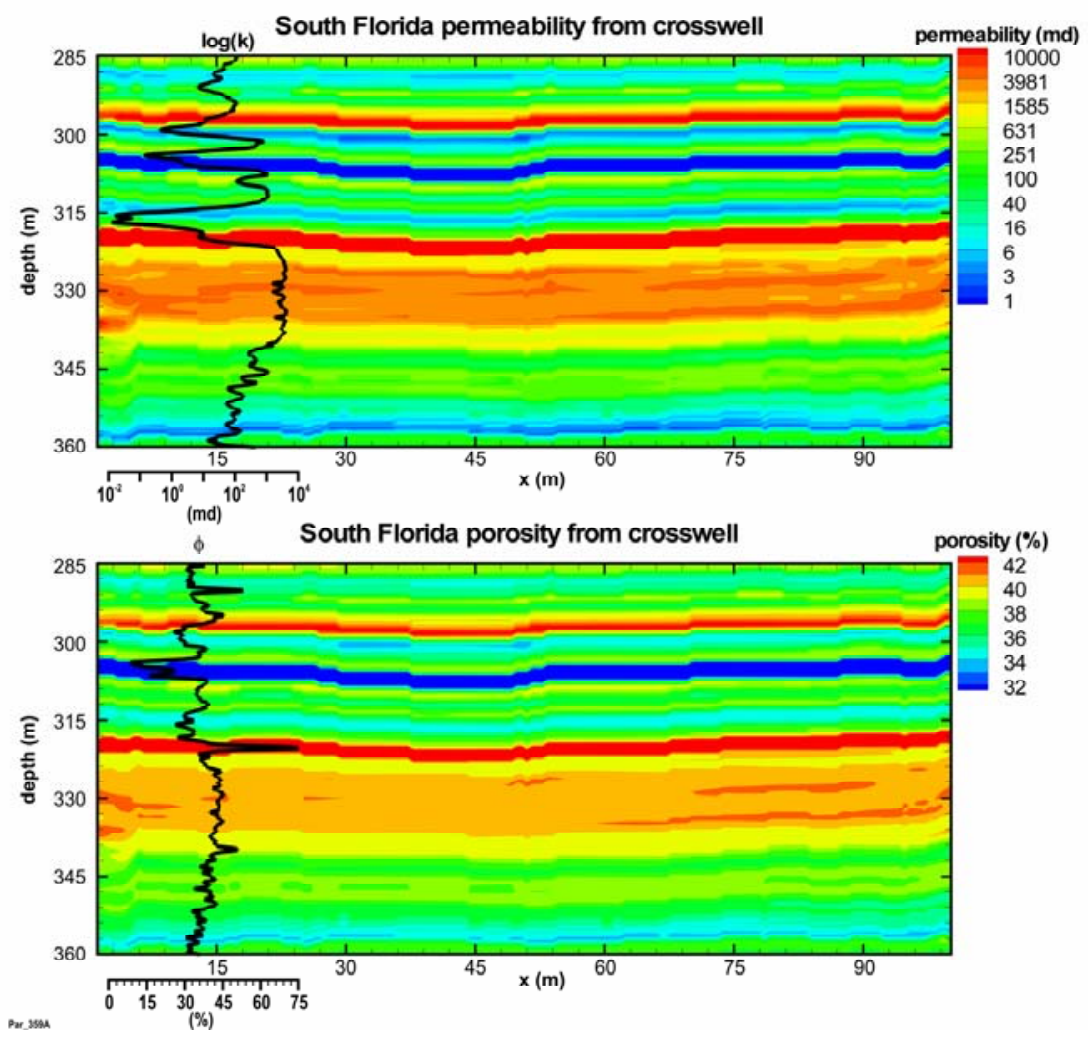

Figure 53. This figure shows the permeability image (top) and porosity image (bottom) computed from the P-wave impedance derived from crosswell seismic data. The respective well logs are superimposed in the images. Although the well where the logs were obtained is located at $x=0 \mathrm{~m}$, the permeability and porosity logs are superimposed at offsets to provide better visual comparison to the permeability and porosity images.

\section{Discussion}

The main objective of this study was to identify a cost-effective method to determine the lateral continuity of hydraulic units identified via the initial test borehole at the Hillsboro site using surface seismic surveys. The optimal subsurface condition for Aquifer Storage and Recovery (ASR) is that hydraulic units are fairly consistent over a lateral extent so that multiple large-capacity wells ( 5 million gallons per day) can be constructed and the individual injected bubbles of water coalesce to form a freshwater reservoir in a brackish water aquifer. The cost of this $800-\mathrm{m}(1 / 2-\mathrm{mile})$ survey was approximately $\$ 50,000$, as compared to $\$ 375,000$ to drill and test a single 20 -cm (8-inch) diameter test hole to a depth of $396 \mathrm{~m}$ (1300 ft.). Thus, this method provided a costeffective way to determine the feasibility of full-scale ASR implementation at the Hillsboro site.

However, the 2D seismic method has some limitations relative to $3 \mathrm{D}$ seismic surveys if it is required to image $3 \mathrm{D}$ features. This situation can occur if the ASR unit is opened in other directions, and as a result of the eventual need to map the 3D flow unit that may be developed. In our experiments, the narrow Hillsboro site did not allow us to consider running a second perpendicular 2D line. 
A potential pitfall of the use of the impedance method can occur in complicated geological environments with strong lateral variations in all directions. If the seismic data does not accurately map the true geology (because of complicated structure, lack of resolution, or poor data), then the impedance calculations may be inaccurate. This can be mitigated by using more wells, which will allow including more well logs for better control but will also increase the total drilling and logging cost. We advise conducting a seismic survey in an area where wells exist for calibration purposes. In this case, the logging cost would be the only major expense.

In contrast to other model-based methods for estimating rock properties (e.g., Yamamoto, 2003) the method presented here is strictly empirical. Model-based methods are limited to the specific situation where the model applies, often regions of very high permeability or carbonate aquifers only. Our data-driven approach assumes no model and is not limited to specific permeability values. The present impedance technique can be used when well log control is available. In complex heterogeneous geology, it requires more well control for imaging the subsurface. In zones where there is no correlation of impedance with porosity or permeability, images cannot be accurately predicted.

\section{E. Conclusions}

A comparison of 2D surface seismic, crosswell seismic, and sonic log data demonstrates that 2D seismic reflection measurements capture several flow units, although at a lower resolution than does crosswell seismic data. However, the permeability and porosity well $\operatorname{logs}$ (overlain on the images) correlate with the permeability and porosity images based on the 2D seismic impedance. In particular, a vuggy carbonate zone is delineated by the permeability images between depths of $323 \mathrm{~m}$ (1060 ft.) and $341 \mathrm{~m}$ (1120 ft.). The combined detectability and resolution obtained with the surface seismic method allow us to characterize the flow units associated with the coherent reflectors observed in the entire original seismic section and its corresponding impedance image. Thus, the continuous reflectors delineate the ASR over a lateral distance of $1067 \mathrm{~m}$ (3500 ft.). This suggests that surface reflection seismic is a practical and economical method for mapping flow units in the deep carbonate aquifer in south Florida. In general, the seismic line data integrated with well logs can be an important and cost-effective technology for delineating flow units in large hydrological areas. The advantage of using surface reflection seismic over crosswell is that only one well and a surface reflection seismic survey are required to cover long distances, assuming that there is not much lateral variation in the geology. On the other hand, crosswell measurements require two closely spaced wells, which may lead to additional costs in hydrogeology regions. For planning purposes where large 2D or 3D seismic surveys will be carried out, we recommend conducting a crosswell survey for a better understanding of the petrophysics at the borehole and crosswell scales. This will allow the interpreter to relate seismic attributes to the rock and fluid properties in the interwell region. 


\section{THEORY FOR ESTIMATING $Q$ FROM SEISMIC REFLECTION DATA}

In reflection data, $Q$ must be computed from the change in spectra of the reflections. As a seismic wave travels through an attenuating medium, the high frequencies are attenuated more quickly than the lower frequencies. (We will assume that $Q$ in the rock is constant over the bandwidth of the source.) Two primary methods have been proposed for computing $Q$ from changes in a signal's spectral characteristics: the log-spectral-ratio (LSR) method, which calculates the reduction in amplitude of each frequency in the spectrum; and the frequency-shift method, which calculates the shift in the peak or centroid of the spectrum (e.g. Quan and Harris, 1995; Zhang and Ulrych, 2002). Both methods assume that attenuation is the only thing that affects the shape of the reflection spectra.

Estimating $Q$ from surface seismic reflection data is much more difficult than computing $Q$ from transmission data, because the reflection coefficient strongly affects the amplitude and is often poorly known. The algorithms for computing $Q$ from reflection data fall into two basic categories: spectral ratio methods and frequency shift methods. Both methods rely on the fact that, for a constant $Q$, higher frequencies are attenuated faster than lower frequencies. Spectral ratio methods compute the decrease in amplitude for each frequency in the reflection; the slope of the decrease versus frequency then determines the $Q$ value. Frequency shift methods look at the peak or center frequency of the reflected pulse rather than the amplitude; this frequency will shift lower as the pulse is attenuated. Using these methods allows the $Q$ estimate to be based on the relative frequency content of the reflected pulse and not its absolute amplitude.

Specific implementations of algorithms are outlined below. For the purposes of this discussion, consider a signal $s(t)$, which at time $t=t_{0}$ has a spectrum $A(f)$. The pulse originates from a point at $t=0$, and propagates through a uniform medium with a constant $Q$. Thus, at time $t$, the signal is given by

$$
s(t)=\frac{1}{t} \int A(f) \exp \left(-i 2 \pi f t^{\prime}\right) \exp \left(-\pi f t^{\prime} / Q\right) d f
$$

while the corresponding amplitude spectrum is

$$
S(f, t)=\frac{1}{t} A(f) \exp \left(-\pi f t^{\prime} / Q\right)
$$

which includes amplitude reduction by both spherical spreading and intrinsic attenuation. Here, $t^{\prime}=t-t_{0}$.

These methods all provide a $Q$ for a uniform, although bounded, layer. The $Q s$ for multiple layers are found by a layer-stripping technique. In this method, the $Q$ of the top layer is solved for directly. Then a combined $Q$ for the top two layers is found, and knowledge of the relative transit times and attenuation in the top layer is used to extract the $Q$ of the second layer. 


\section{A. Dasgupta and Clark (1998)}

Dasgupta and Clark use the spectral ratio method. Dividing the amplitude spectra at time $t_{1}$ by that at time $t_{0}$ and taking the natural logarithm,

$$
\ln \left(\frac{S\left(f, t_{1}\right)}{S\left(f, t_{0}\right)}\right)=\ln \left(\frac{t_{0}}{t_{1}}\right)-\frac{\pi f}{Q}\left(t_{1}-t_{0}\right) .
$$

Thus, the log-spectral-ratio between two times is a linear function of frequency with slope proportional to $1 / Q$. If the signal at time $t_{1}$ is a reflected signal, then the spectral amplitude is multiplied by the reflection coefficient $R$ :

$$
\ln \left(\frac{S\left(f, t_{1}\right)}{S\left(f, t_{0}\right)}\right)=\ln \left(\frac{R t_{0}}{t_{1}}\right)-\frac{\pi f}{Q}\left(t_{1}-t_{0}\right) .
$$

This results in a change to the intercept of the linear function but not the slope. Therefore, this method may be used to determine $Q$ from reflection data.

In an interesting application, Dasgupta and Clark apply this method to each trace in a CMP NMO pre-stack gather. They then plot $1 / Q$ versus offset squared and fit a line to determine the $Q$ at zero offset. By using data from several traces, all of which have different shot points and receiver locations, they can obtain a more reliable estimate of $Q$.

\section{B. Quan and Harris (1995)}

Quan and Harris apply the frequency shift method to crosswell transmission data. Although this is transmission and not reflection data, the frequency shift method should also be applicable to reflection data sets. Their particular implementation, later slightly expanded upon by Neep et al. (1996), is based on the centroid frequency. Given a pulse spectrum of $S(f, t)$, the centroid frequency is defined as

$$
f_{c}(t)=\frac{\int f S(f, t) d f}{\int S(f, t) d f} .
$$

The method also depends on the spectral variance, which is related to the bandwidth of the pulse:

$$
v(t)=\frac{\int\left(f-f_{c}\right)^{2} S(f, t) d f}{\int S(f, t) d f} .
$$

Given that the spectrum changes with time as in Equation (37), the shift in the centroid frequency between times $t_{1}$ and $t_{2}$ is 


$$
f_{c}\left(t_{2}\right)-f_{c}\left(t_{1}\right)=-\frac{g\left(t_{2}-t_{1}\right) v\left(t_{1}\right)}{Q},
$$

where $g$ is a factor which depends on the shape of the spectrum at time $t_{1}$. If the spectrum is Gaussian at time $t_{1}$, then $g=\pi$.

A problem with this method is the need for prior knowledge of the pulse spectral shape to determine the coefficient $g$.

\section{Zhang and Ulrych (2002)}

Zhang and Ulrych explicitly extend the central frequency method of Quan and Harris to reflection data, modifying the formulation slightly to focus on the peak frequency rather than the central frequency. Assuming that the spectrum of the deconvolved wave is a Ricker wavelet, they provide a relationship between the shift of the peak frequency and the $Q$ value.

The spectrum of a Ricker wavelet with primary frequency $f_{0}$ after traveling for some time $t$ in an attenuating medium is

$$
S(f, t)=\frac{2}{\sqrt{\pi}} \frac{f^{2}}{f_{0}^{2}} \exp \left(-\frac{f^{2}}{f_{0}^{2}}\right) \exp \left(-\frac{\pi f t}{Q}\right)
$$

The peak frequency, $f_{\mathrm{p}}$, is found by taking the derivative with respect to frequency and setting the result equal to zero. The resulting equation relates the shift in peak frequency to $Q$ by

$$
Q=\frac{\pi t f_{p} f_{0}^{2}}{2\left(f_{0}^{2}-f_{p}^{2}\right)}
$$

Since the peak frequency of the source pulse is often unknown, $f_{0}$ may be estimated by measuring $f_{\mathrm{p}}$ for the same reflection at different offsets and requiring the resulting $Q$ to be the same as well. Thus,

$$
f_{0}=\sqrt{\frac{f_{p 1} f_{p 2}\left(t_{2} f_{p 1}-t_{1} f_{p 2}\right)}{t_{2} f_{p 2}-t_{1} f_{p 1}}} .
$$

The disadvantages of this method are the assumption of a particular form for the wavelet spectrum (although many deconvolved seismic pulses resemble Ricker wavelets) and the use of the spectrum peak frequency, which may be obscured by noise or scalloping in the spectrum. Also, the peak frequency is more sensitive to the discrete nature of the Fourier transform. If short time windows are needed, then the frequency resolution could be less than or similar to the frequency shift. It is difficult to accurately measure a $12 \mathrm{~Hz}$ frequency shift for example, when you're limited to $5 \mathrm{~Hz}$ frequency resolution. 


\section{Barnes (1993)}

Barnes (1993) suggests that, instead of the peak or centroid frequency, the instantaneous frequency may be used to determine $Q$ effects on waveforms. To review, the instantaneous attributes (Taner et al., 1979) of a signal, $s(t)$, may be found by assuming the signal is composed of an amplitude envelope, $A(t)$, and a time-varying phase, $\phi(t)$ :

$s(t)=A(t) \cos (\phi(t))$.

While this decomposition would appear to be severely underdetermined, it is in fact well constrained by requiring that the envelope amplitude is non-negative and that the phase is monotonically increasing with time:

$$
\begin{aligned}
& A(t) \geq 0 \\
& \frac{d \phi}{d t} \geq 0
\end{aligned}
$$

Under such conditions, the amplitude function may be constructed from the real signal, $s(t)$, and its Hilbert transform, $s^{*}(t)$, as

$$
A(t)=\sqrt{s^{2}(t)+s^{* 2}(t)} .
$$

The quantity $\mathrm{d} \phi / \mathrm{dt}$ is then the instantaneous circular frequency. This may be justified by considering that a sine wave of frequency $\omega$ and amplitude $A_{0}$ has a decomposition of $A(t)=A_{0}$ and $\phi(t)=\omega t-\pi / 2$.

For computing $Q$, Barnes suggests a method similar to that of Quan and Harris (1995), using the instantaneous frequency and the instantaneous bandwidth instead of the spectrally weighted quantities. The instantaneous frequency was defined above; the instantaneous bandwidth, $\sigma_{\mathrm{f}}$, is defined by

$$
\sigma_{f}^{2}=\left[\frac{1}{2 \pi A(t)} \frac{d A(t)}{d t}\right]^{2}
$$

Since all relevant quantities are now available on a sample-by-sample basis, rather than averaged over a time window, an instantaneous $Q$ may also be defined:

$$
Q_{i}(t)=\pi f_{i}(t)\left(\frac{1}{A(t)} \frac{d A}{d t}\right)^{-1}
$$

A problem with this method is that the $Q$ values are based primarily on the rate of change of the amplitude envelope. This creates difficulties with reflection data, because the amplitude envelope is primarily controlled by the reflection coefficient, and only secondarily affected by the attenuation. In cases where the reflection coefficient magnitudes are not uniform, the instantaneous $Q$ will bear little relation to the true $Q$. 


\section{ALGORITHM FOR $Q$ ESTIMATION WITH THIN-BED CORRECTIONS}

Many sedimentary formations the reflection spectra have a significant component from interfering closely spaced reflections. This gives rise to the well-known tuning effect (e.g., Sheriff and Geldart, 1995), in which amplitudes may be increased or decreased by constructive or destructive interference from reflectors less than a quarterwavelength apart. In addition to the amplitude changes, however, there is also a change in the apparent frequency and phase of the reflected wave, as noted by Robertson and Nogami (1984) as well as Raikes and White (1984). In this section, we examine how the changes in the apparent frequency due to thin beds affect the spectrum of the reflected wave, and in turn, affect the computation of $Q$ from seismic reflection data. We mention here that we are not concerned with analyzing or interpreting the absorption due to short period multiples (e.g., O'Doherty and Anstey, 1971) but rather with correcting for the influence of local thin bed primaries on the windowed reflection spectrum. We combine a method for using well log data to correct for the thin bed effect (Raikes and White, 1984) with the Q-versus-offset (QVO) method of Dasgupta and Clark (1998), and demonstrate its successful application in computing $Q$ from both synthetic and real surface seismic data.

Though we compute $Q$ as a function of CDP using the QVO method, we also employ both the LSR method and the centroid frequency shift method (CF) as described in Quan and Harris (1995) to evaluate the spectral changes. The peak frequency shift method, applied to surface seismic data in Zhang and Ulrych (2002), was also tried, but found to be too sensitive to noise and other perturbations to the spectrum. Both the LSR and CF methods depend on comparing the spectrum of the target signal with a reference spectrum. The reference spectrum is ideally that of the source; but since that is often unavailable, a shallow strong reflection may be substituted. Briefly, the QVO calculation involves taking post-NMO CDP gathers, stacking several adjacent offsets within each CDP gather to improve the signal to noise ratio, computing $Q$ for each offset stack within each CDP (using LSR and/or CF), and extrapolating the resulting $Q$ values to zero offset for each CDP using a least squares fit. The resulting $Q$ indicates the attenuation between the reference reflector and the target reflector.

One difficulty with computing $Q$ from surface seismic data is that both the LSR and CF methods for determining $Q$ were originally derived for use with transmission data rather than reflection data. The recorded signal in a transmission experiment is the directly transmitted pulse plus a coda of forward scattered energy. Since the coda is necessarily delayed with respect to the directly transmitted wave, the two may be at least partially separated with time gating. This results in a relatively robust estimate of the transmitted pulse spectrum. Ganley and Kanasewich (1980) examined how thin-bed multiples affect the LSR method for transmitted waves, and proposed a correction based on synthetic seismograms.

In reflection data, the reflected wave train is the convolution in the time domain of the apparent source wavelet with the reflection spike time series "stickogram" (Sheriff and Geldart, 1995). Since convolution in the time domain is equivalent to multiplication in the frequency domain (Oppenheim and Schafer, 1989), the reflected pulse spectrum can be distorted by closely spaced reflectors. Figure 54 demonstrates this effect. For the single isolated reflector shown in Figure 54(a), no spectral distortion is seen. For the three 
evenly spaced reflectors shown in Figure 54(b), with the same total reflectivity as Figure 54(a), the spectrum of the reflected wave is enhanced near $50 \mathrm{~Hz}$ and suppressed near 20 and $80 \mathrm{~Hz}$. This effect arises from constructive and destructive interference of the reflections at certain frequencies. Finally, if there are many closely but randomly spaced reflectors, as in Figure 54(c), the reflected spectrum may be affected irregularly. It could suppress low frequency content in the combined reflection, as in this case, which gives the appearance of negative $Q$ through increased amplitude at high frequencies.
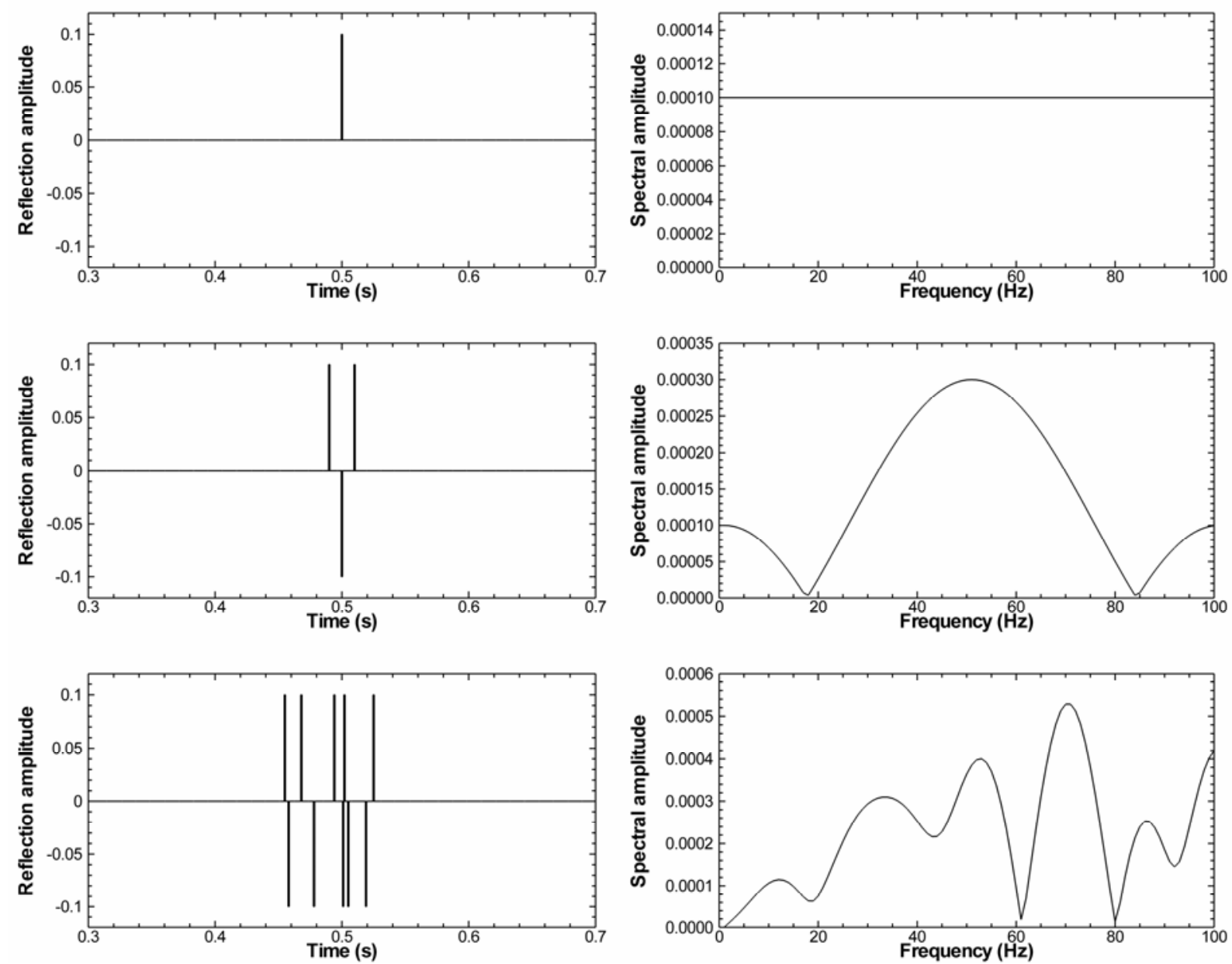

Figure 54. Three hypothetical spike reflectivity stickogram sequences and their corresponding spectra. The reflected pulse spectrum will be the product of the incident pulse spectrum and the spectrum of the spike sequence.

Raikes and White (1984) and White (1992) discuss a method for correcting for these effects, which we will outline here. Consider a source pulse $s(t)$, with corresponding spectrum $S(\omega)$. (We will use a convention of lower case letters denoting time-domain data and upper case letters denoting the equivalent frequency domain data.) The source pulse travels through a layered earth, for which the reflectivity stickogram $r(t)$ is the reflection coefficient at two-way traveltime $t$, and $g(t)$ describes the geometric spreading 
loss in amplitude. If the layered medium is elastic, the resulting observed (primariesonly) reflected signal, $a(t)$, is given by

$$
a(t)=g(t) \int_{-\infty}^{\infty} s(\tau) r(t-\tau) d \tau
$$

In practice, the source pulse is finite so only a small range of $\tau$ contributes to the integral. The convolution operation is linear time-invariant (Oppenheim and Schafer, 1989), so we may take a subset of reflectors, $r_{1}(t)$, which lie near two-way time $t_{1}$, and the local reflected wavetrain contribution from these reflectors will be

$a_{1}(t)=g(t) \int_{-\infty}^{\infty} s(\tau) r_{1}(t-\tau) d \tau$

Then, the local spectrum of the reflected pulse near time $t_{1}, A_{1}(\omega)$, is approximately equal to

$$
A_{1}(\omega)=g\left(t_{1}\right) S(\omega) R_{1}(\omega)
$$

where $R_{1}(\omega)$ is the Fourier transform of $r_{1}(t)$.

If the layered medium is attenuating, with constant $Q$, then the spectrum will be reduced in a frequency-dependent manner such that

$$
A_{1}(\omega)=g\left(t_{1}\right) S(\omega) R_{1}(\omega) \exp \left(-\frac{\omega t_{1}}{2 Q}\right)
$$

Similarly, the local subset of reflectors $r_{2}(t)$ near two-way time $t_{2}$ will generate a reflected pulse with spectrum $A_{2}(\omega)$. Taking the logarithm of the ratio of $A_{2}$ and $A_{1}$,

$$
\ln \left(\frac{A_{2}(\omega)}{A_{1}(\omega)}\right)=\ln \left(\frac{g\left(t_{2}\right)}{g\left(t_{1}\right)}\right)+\ln \left(\frac{R_{2}(\omega)}{R_{1}(\omega)}\right)-\frac{\omega}{2 Q}\left(t_{2}-t_{1}\right)
$$

The first term on the right hand side is independent of frequency. If $R_{1}(\omega)$ and $R_{2}(\omega)$ are both independent of frequency (as they would be if $r_{1}(t)$ and $r_{2}(t)$ represent single, isolated reflectors), then the second term on the right hand side is also constant and $Q$ may be computed from the slope of the log-spectral ratio of $A_{2} / A_{1}$. This is exactly the traditional LSR method. If $r_{1}(t)$ or $r_{2}(t)$ represents a group of closely spaced reflectors, then the second term on the right hand side will vary with frequency, and the slope of the LSR will not be directly proportional to $1 / Q$.

When well log impedance data is available, we in principle should be able to compute the $r(t)$ associated with a particular time window, and hence derive the $R(\omega)$ that is needed to correct the spectral ratio. This should work at least for seismic traces near 
the well, where lateral variations in impedance or dipping beds will not have significantly altered the $r(t)$ profile. For such a case,

$$
\ln \left(\frac{A_{2}(\omega) / R_{2}(\omega)}{A_{1}(\omega) / R_{1}(\omega)}\right)=\ln \left(\frac{g\left(t_{2}\right)}{g\left(t_{1}\right)}\right)-\frac{\omega}{2 Q}\left(t_{2}-t_{1}\right),
$$

and we may again compute $Q^{-1}$ from the slope of the known values on the left hand side of the equation. Note that the $A(\omega) / R(\omega)$ is a local spectrum corrected for the primariesonly thin-bed effects, and as such may also be used in frequency-shift methods for computing $Q$.

Given seismic data consisting of NMO-corrected CDP gathers processed without any kind of spectral balancing and a well log converted to reflectivity stickogram in twoway time, our method is to:

(1) Select a reference reflection and one or more target reflections.

(2) Apply a tapered time window such as the Hanning window (Oppenheim and Schafer, 1989) to each trace in the seismic data and the reflectivity stickogram. The time window should be short enough to select the reflection of interest, but long enough that the frequency resolution (equal to the inverse of the time window length) is sufficiently smaller than the bandwidth of the pulse. The tapered time window gradually reduces the influence of reflectors away from the two-way time of interest.

(3) Take the Fourier transform of each time window, and divide the seismic spectrum amplitude by the amplitude of the reflectivity stickogram spectrum. We recommend smoothing the stickogram spectrum before the division since it often contains zeros. These zeros result from assuming planar, laterally invariant reflectors and wave propagation at normal incidence. In reality, or even in multidimensional numerical simulation, these frequencies will correspond to spectral minima, but not true zeros.

(4) Using the target reflection local corrected spectrum and the reference reflection local corrected spectrum, compute $Q$ using the LSR and CF methods. Following the QVO method of Dasgupta and Clark (1998), these values may be extrapolated to zero offset within each CDP, producing an average $Q$ value for each CDP for each target reflection. 


\section{SYNTHETIC TEST DATA}

To test this method, we first used a synthetic data example consisting of traces from a viscoelastic 2-D finite difference model. The model geometry consists of five layers (four reflectors) each with different $Q$ and P-velocity (Figure 55). The model density and S-wave velocity are kept constant to eliminate any S-wave reflections that might interfere with the recorded signal. The 2-D model consists of a staggered grid with normal stresses at cell centers, densities and displacements at cell faces, and shear stress at cell corners. The cells have 1-m spacing, and there are 2100 grid cells horizontally and 4500 grid cells vertically. The source pulse is a Ricker wavelet of $100-\mathrm{Hz}$ peak frequency, and the time step is 0.25 ms. The absorbing boundary condition of Higdon (1991) is used to reduce reflections from the domain boundary, although the boundaries are placed sufficiently far from the source and receivers that no significant reflection would be detected regardless. We use two variations on this model. In one case, there are no thin beds, while in the second case there are two thin beds added at each primary layer boundary, as shown in Figure 55.
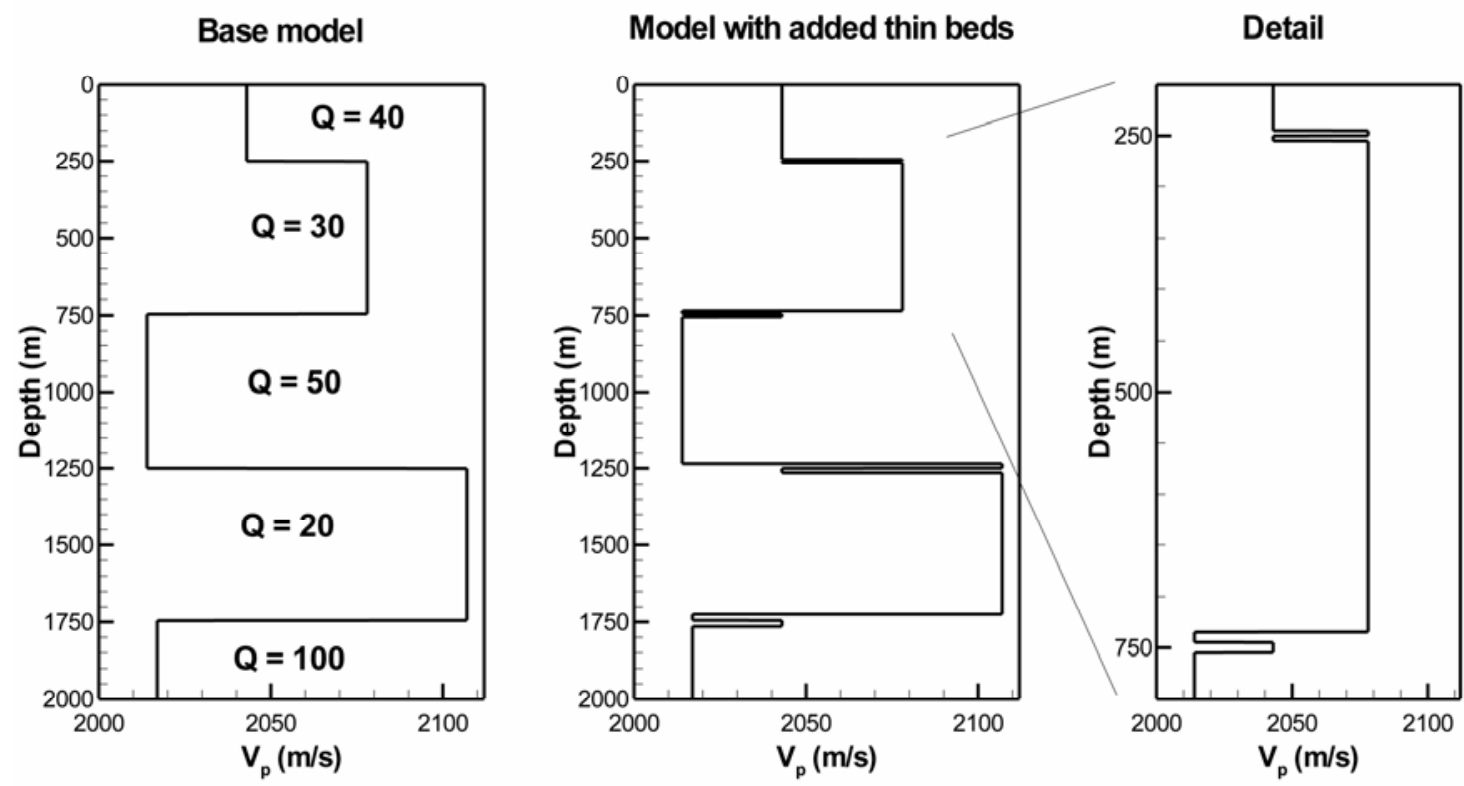

Figure 55. Models for synthetic seismic reflection data without and with thin beds added near each main layer boundary.

Since there is no lateral variation in the model geometry, a single shot suffices to generate all necessary offsets. The resulting seismic data is shown for each case (processed with automatic gain control only) in Figure 56.

For cases of no thin beds and thin beds added, we use the first reflection as the reference and compute the average $Q$ between that and each later reflection, using both the uncorrected and corrected spectra when thin beds are added. The results are shown in Table 8. 

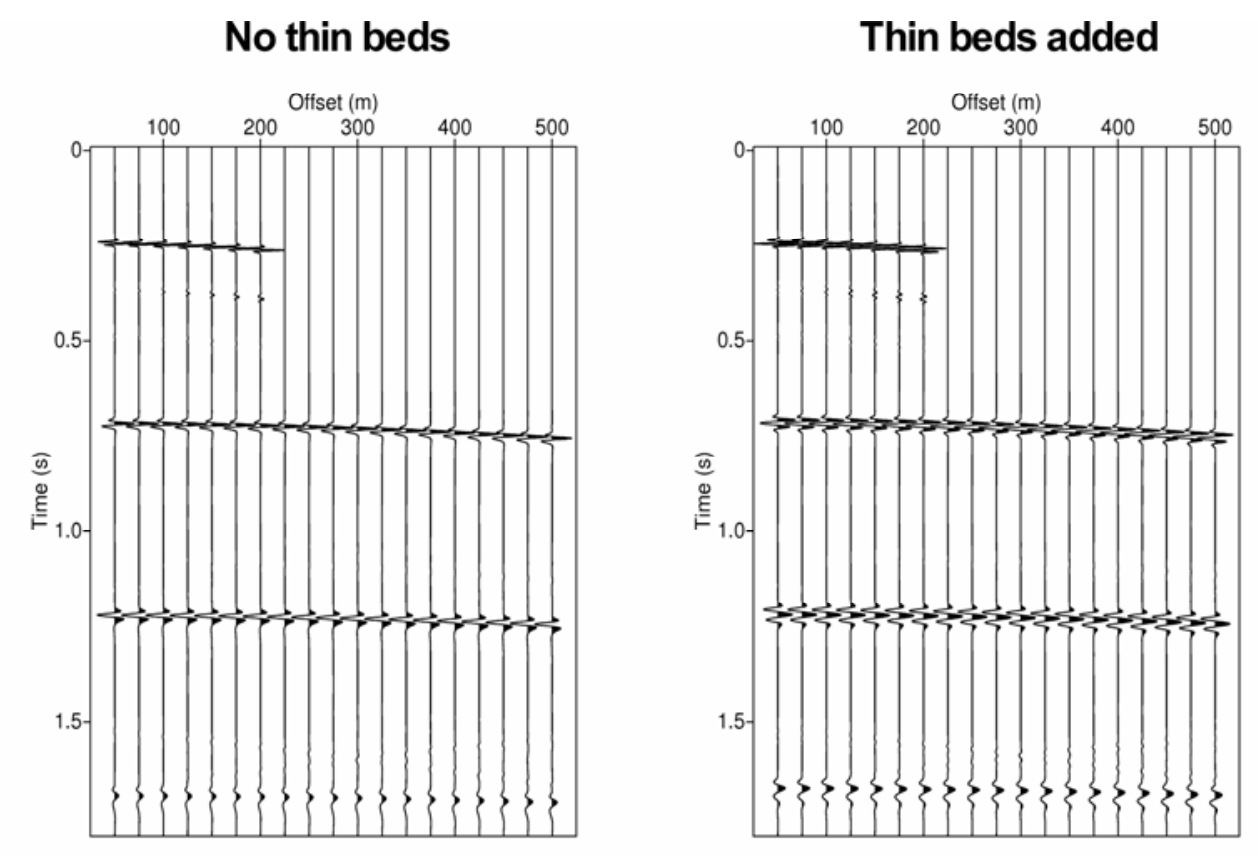

Figure 56. Seismic reflection response of synthetic models without (left) and with (right) thin beds near each main layer boundary. Seismograms are shown with AGC, since attenuation strongly reduces the amplitude of the later reflections. The low-amplitude reflections following the first reflection are $P-S$ and $S-S$ converted waves. The signals from the first reflector are muted after $200 \mathrm{~m}(656 \mathrm{~m})$ offset because of interference from the direct wave.

Table 8. True and Computed Values of $Q$ from the Synthetic Test Data

\begin{tabular}{|l|l|l|l|l|}
\hline & true value & $\begin{array}{l}\text { no thin } \\
\text { beds }\end{array}$ & $\begin{array}{l}\text { thin beds added } \\
\text { (uncorrected) }\end{array}$ & $\begin{array}{l}\text { thin beds added } \\
\text { (corrected) }\end{array}$ \\
\hline $\begin{array}{l}\text { QLSR reference to } \\
\text { reflection 2 }\end{array}$ & 30 & 32 & 14 & 33 \\
\hline $\begin{array}{l}\text { QLSR reference to } \\
\text { reflection 3 }\end{array}$ & 38 & 42 & 14 & 43 \\
\hline $\begin{array}{l}\text { QLSR reference to } \\
\text { reflection 4 }\end{array}$ & 29 & 32 & 26 & 29 \\
\hline$Q_{3}$ from layer stripping & 50 & 57 & 15 & 61 \\
\hline$Q_{4}$ from layer stripping & 20 & 22 & -38 & 18 \\
\hline
\end{tabular}


Based on this data, a layer-stripping method (Dasgupta and Clark, 1998) provides the $Q$ of each individual layer as well. Time windows are 0.145 to $0.345 \mathrm{~s}$ for the first (reference) reflection, 0.528 to $0.908 \mathrm{~s}$ for the second reflection, 0.97 to $1.47 \mathrm{~s}$ for the third reflection, and 1.54 to $1.84 \mathrm{~s}$ for the fourth reflection. Only the LSR method is used in this synthetic example, because the large frequency shift between the first and fourth reflections invalidates some assumptions of the CF method. Since this is an almost ideal data set, it is not surprising that with no thin beds present, the QVO method recovers the $Q$ of each layer almost exactly. The thin beds at the layer boundaries of the second model, however, interfere with the reflection spectra in the manner discussed above, so that the uncorrected QVO method $Q$ values are quite poor. Applying the well-log-based spectral correction substantially removes this effect, and the corrected $Q$ values are of reasonable accuracy.

The uncorrected and corrected normalized local spectra are shown in Figure 57 for each of the four primary reflections. The dotted line is the uncorrected spectrum for reflections including thin beds, while the solid line is the spectrum with no thin beds. The latter is a close approximation of the actual spectrum of the incident wave when thin beds are present; the thin beds introduce complexity in the reflected waveform, but because of the low reflection coefficient do not significantly impact the transmitted wave.
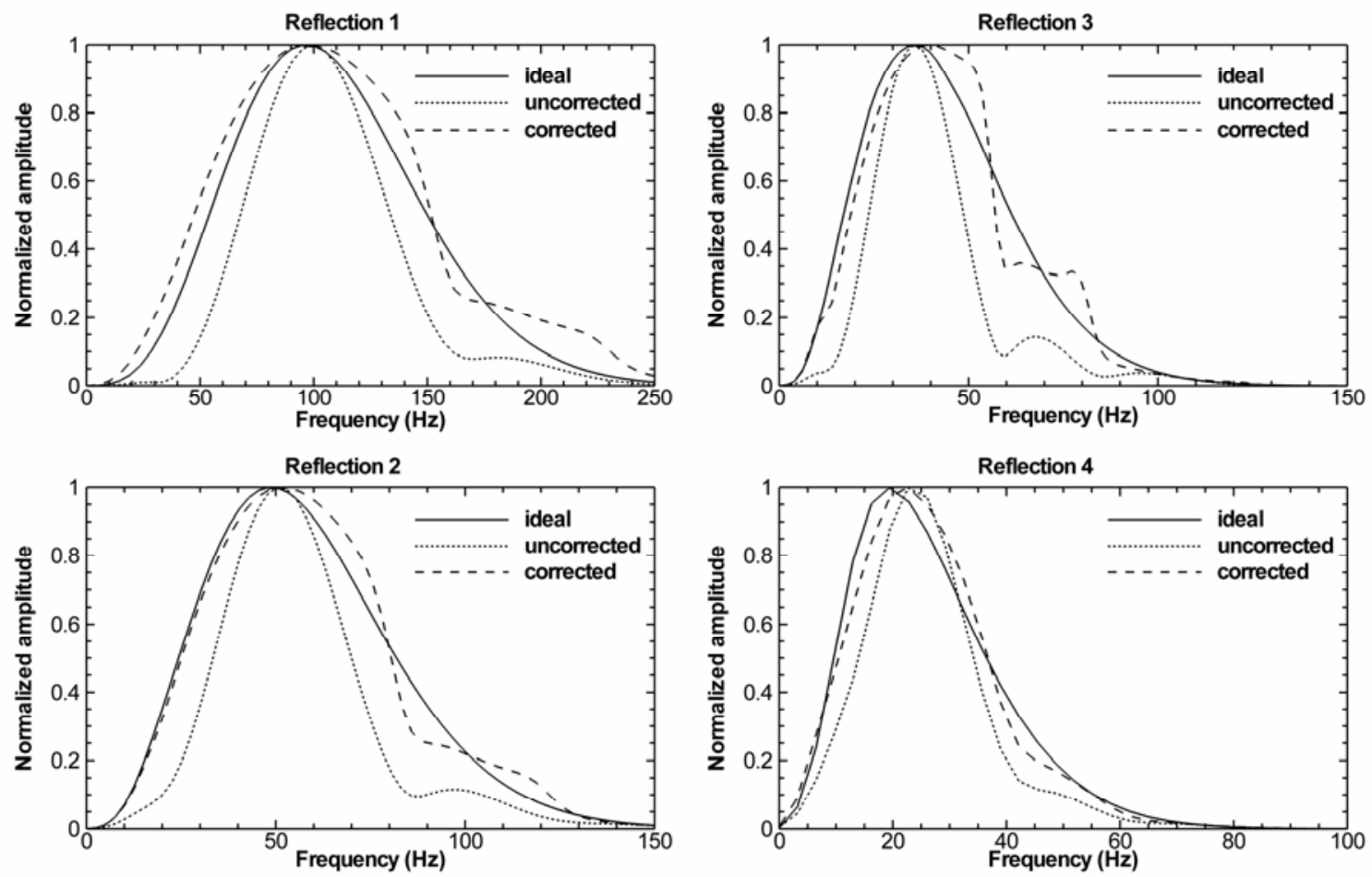

Figure 57. Comparison between uncorrected and corrected normalized local reflection spectra. In each figure, the solid line is the spectrum if there are no thin beds. The dotted line is the spectrum with thin beds present. The dashed line is the corrected spectrum using the well log deconvolution method. Ideally, the dashed line would coincide with the solid line. The corrected spectrum is, however, a definite improvement over the uncorrected spectrum. Note that each frequency axis spans a different range. 
The dashed line is the corrected spectrum for each case. Ideally, the corrected spectrum (dashed line) would exactly match the incident spectrum (solid line). This does not happen because we have imperfectly smoothed out the zeros in the spectrum of the reflectivity stickogram. Nevertheless, the corrected spectrum is an improvement over the uncorrected spectrum, and the differences between corrected and incident spectra appear to average out over a range of frequencies.

The correction method appears to be robust in the presence of moderate amounts of noise and uncertainty. One of the major sources of uncertainty for this technique is the possibility of errors in the well logs. Errors in the depth-to-time tie between well log and seismic data are not overly significant since a shift of a signal in time only affects the phase of the spectrum and not the amplitude. Time shifts may become important if strong reflectors are moved into or out of the time window of the Fourier transform. The use of a tapered windowing function such as the Hanning window (Oppenheim and Schafer, 1989) can minimize the problem for small time shifts.

Errors in the well log values are more significant, since they directly alter the reflectivity stickogram used to compute the corrected spectra. To evaluate this effect, we introduced uniform Gaussian noise to the reflectivity stickogram at levels of 1, 2, 5, and $10 \%$, and reprocessed the spectrally corrected $Q$ values for eight realizations of each noise level. The resulting average $Q$ values and their standard deviations are given in Table 9. With increasing well log error, there is a trend toward increasing uncertainty in $Q$ and a trend toward (in this case) lower $Q$. The latter follows from Equation (43): as the noise level in the reflectivity stickograms increases, the spectra become dominated by the noise and both $R_{1}$ and $R_{2}$ approach the noise spectrum. On average, $R_{1}$ and $R_{2}$ cancel out, and the mean $Q$ approaches that of the uncorrected spectrum (see Table 8). When the noise added to the reflectivity stickogram reaches $5 \%$, the added error begins to overtake the inherent uncertainty in the calculation, and there is notable deterioration in the accuracy of the spectrally corrected $Q$ factor.

Table 9. Effect of Well Log Error on Uncertainty of Computed, Spectrally Corrected $Q_{L S R}$ Values

\begin{tabular}{|l|l|l|l|l|l|l|}
\hline & true value & $0 \%$ error & $1 \%$ error & $2 \%$ error & $5 \%$ error & $10 \%$ error \\
\hline $\begin{array}{l}\text { reference to } \\
\text { reflection 2 }\end{array}$ & 30 & 33 & $33 \pm 4$ & $34 \pm 8$ & $29 \pm 6$ & $16 \pm 4$ \\
\hline $\begin{array}{l}\text { reference to } \\
\text { reflection 3 }\end{array}$ & 38 & 43 & $43 \pm 3$ & $40 \pm 6$ & $32 \pm 6$ & $26 \pm 5$ \\
\hline $\begin{array}{l}\text { reference to } \\
\text { reflection 4 }\end{array}$ & 29 & 29 & $30 \pm 4$ & $26 \pm 5$ & $22 \pm 5$ & $24 \pm 5$ \\
\hline
\end{tabular}

Note: Average values are presented along with the standard deviations derived from eight realizations of each degree of error in the reflectivity stickogram. 


\section{APPLICATION TO FLORIDA SITE (REAL DATA)}

Having successfully demonstrated the correction method in the idealized model data, we now turn to an example using real data. To leverage existing data from the south Florida carbonate aquifer near West Palm Beach, we were able to acquire 2-D seismic profiles of P- and S-wave data at the same location at minimal cost to the project in cooperation with the South Florida Water Management District and Bay Geophysical. Existing data from this site already at SwRI includes sets of logs from two wells, extensive core information, and a high-resolution crosswell data set. Full-waveform sonic log data from one of these wells is currently being used in the development of a borehole-scale sonic-frequency $Q$ inversion process.

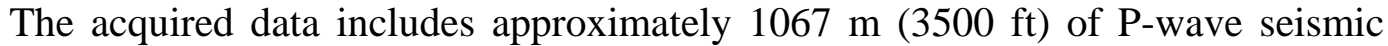

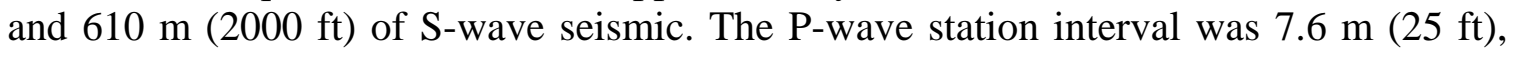
while the S-wave station interval was $5 \mathrm{ft}$. A mechanical vibrator source was used, with frequency sweep up to $300 \mathrm{~Hz}$. We obtained data from the contractor in both migrated and prestack form. To aid in later processing for attenuation, we also obtained prestack data with frequency-independent processing. The migrated data, showing many predominantly planar reflectors, is shown in Figure 58, along with well logs.

An example of a prestack NMO gather is shown in Figure 59. Most of the reflectors are clearly visible on this gather, as is the low-frequency ground roll (Rayleigh wave) and high-frequency airblast.

Since there is little visible lateral variation, we expect the well log correction technique to be applicable over most of the seismic line. Synthetic seismograms show a good tie between the well log (near CDP 50) and the actual seismic data. From this data, we take the reference reflection time window to extend from 0.15 to 0.20 seconds, and choose two target time windows: 0.30 to 0.36 seconds and 0.46 to 0.56 seconds. These times cover roughly the entire range of the well log. 

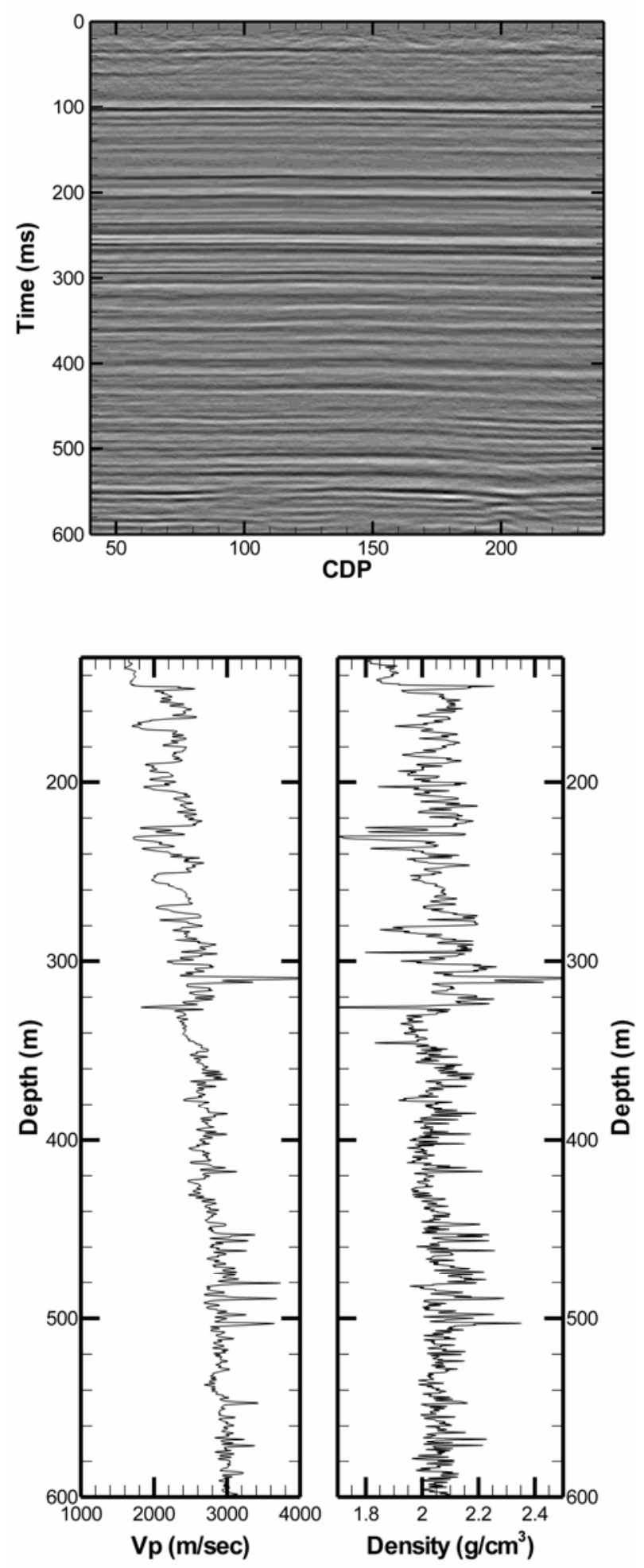

Figure 58. Migrated seismic data and well logs from the Florida aquifer test site. 


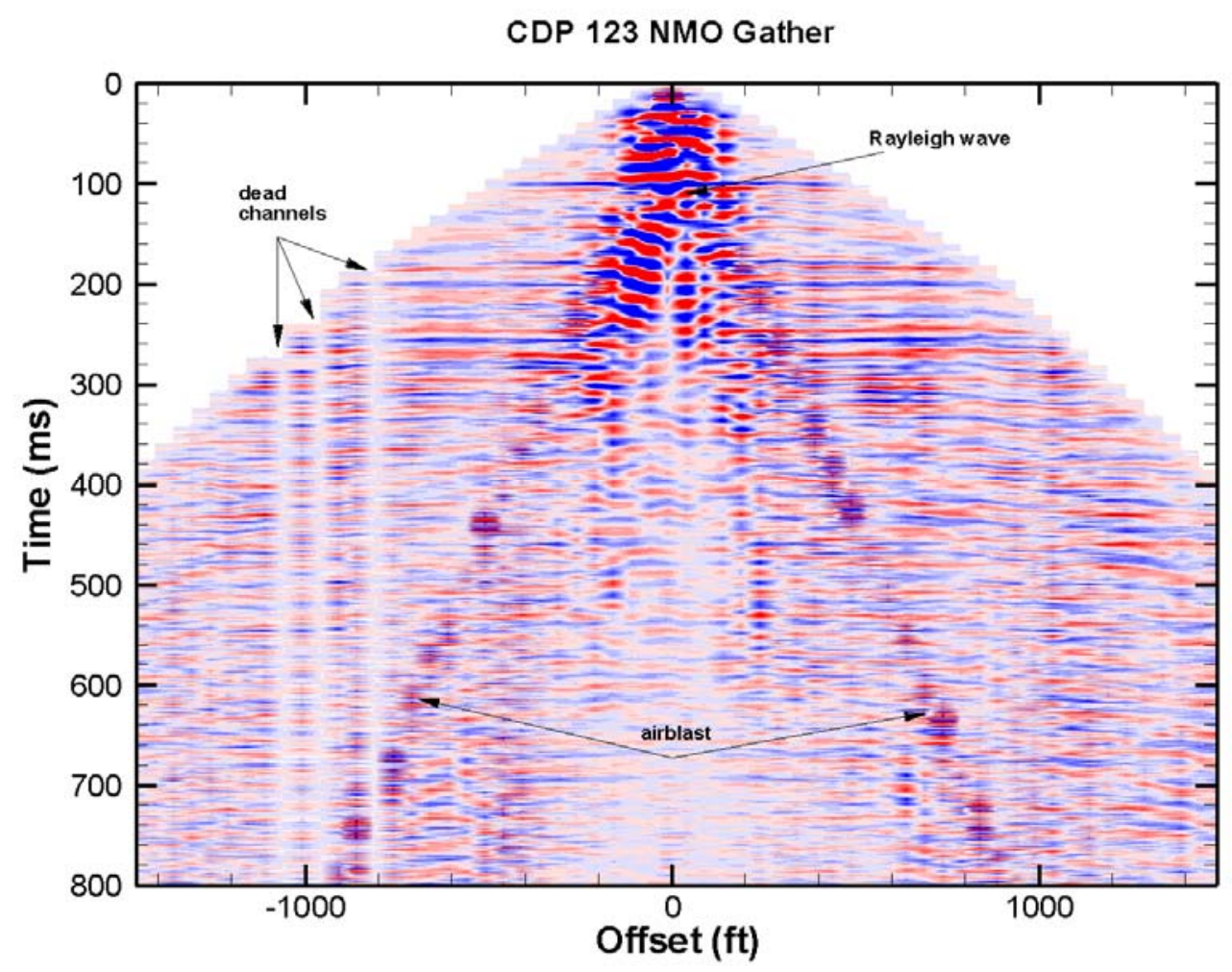

Figure 59. An unmuted pre-stack CDP NMO gather from the South Florida surface seismic data set. The ground roll (Rayleigh wave) and airblast are visible on these raw data traces.

Using the spectral correction technique along with the QVO calculation significantly enhances the stability of the computation of $Q$. At the top of Figure 60, we show the uncorrected and corrected log-spectral ratios as a function of frequency for one 5-trace offset stack from a CDP in the middle of the spread, using the reference and first target time windows. Small shifts in the spectral amplitude at a few frequencies make a large difference in the resulting straight-line least-squares fit. The LSR profile using corrected spectra much more closely matches a straight line, and produces a rather different (and more realistic) slope. The bottom plots of Figure 60 show the computed $Q^{-1}$ for each offset stack of this CDP.

Here the LSR results are shown as square symbols with error bars and the CF results are shown as triangles. The spectral corrections not only produce a more consistent value of $Q$ for each offset, but also reduce the statistical uncertainty computed from the least-square fit to the LSR profile. 

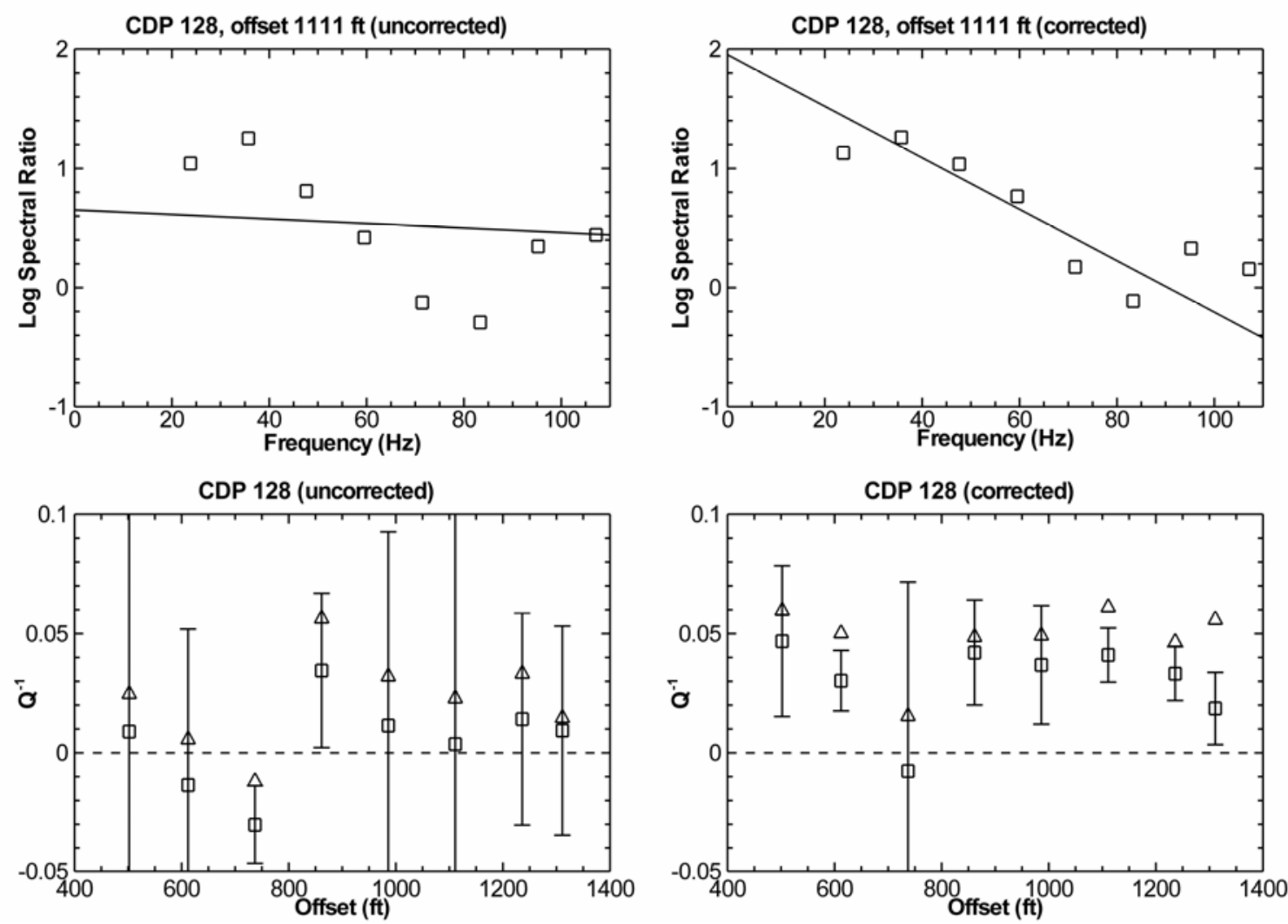

Figure 60. (top) Plots of corrected and uncorrected log-spectral-ratio as a function of frequency for CDP 128, offset $1111 \mathrm{ft}$. in the Florida data. (bottom) $Q^{-1}$ versus offset plots for CDP 128 of log-spectral-ratio $Q^{-1}$ (square symbols with error bars) and centroid-frequency $Q^{-1}$ (triangles). This data is using the 0.18-s and 0.34-s window centers.

Figure 61 shows the uncorrected (left) and spectrally corrected (right) $Q$ estimates as a function of CDP using both the LSR and CF methods. Here, we plot the average $Q^{-1}$ and the standard deviation within each group of 10 CDPs. The computed $Q$ from the shallower reflection target is highly variable in the uncorrected case, with many negative $Q$ values. After applying the spectral correction, the $Q$ values are much more stable, and seem to have an average value of $Q^{-1} \sim 0.03(Q \sim 33)$ using the LSR method and $Q^{-1} \sim$ $0.05(Q \sim 20)$ using the CF method. Within the given range of uncertainty, these values hold fairly constant along the length of the line. Apart from a few faults and some small changes in bed thickness, the rock properties at this site are expected to be laterally invariant. For the deeper target reflection, there is not so much change between the uncorrected and corrected $Q$ estimates. The spectrally corrected $Q$ estimate is definitely somewhat improved, however, in terms of fewer negative $Q$ values and slightly less variability. (Negative $Q$ values are physically unrealistic.) Again, we see that $Q^{-1} \sim 0.03$ $(Q \sim 33)$ across the length of the line. We would expect the deeper reflection target to produce more stable $Q$ values because of the longer travel time. In this case, the LSR and CF methods produce similar results, which give us additional confidence in the computed $Q$ values. 

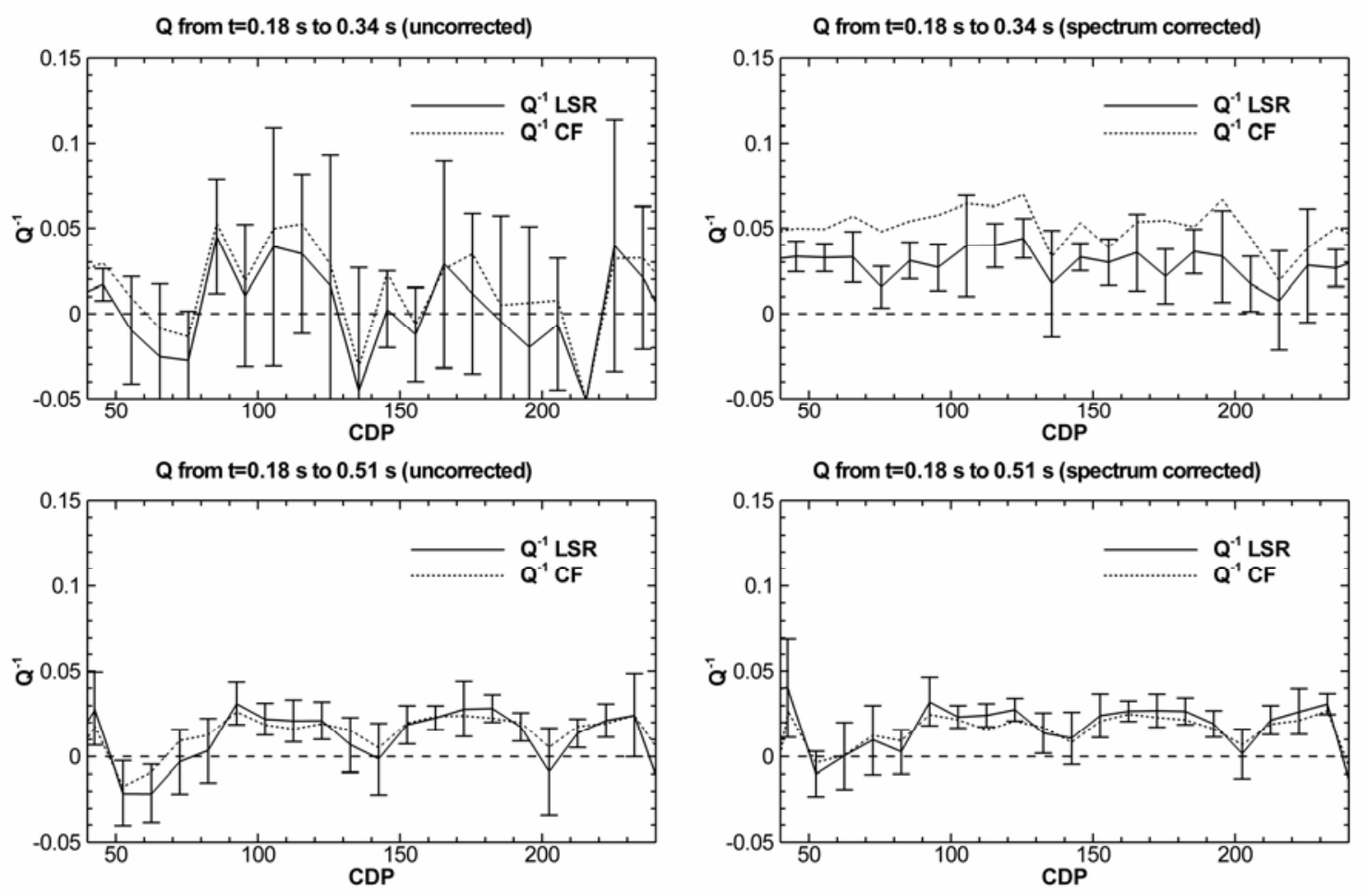

Figure 61. Comparison of uncorrected (left) and corrected (right) $Q^{-1}$ values computed from two sets of reflections from the Florida seismic line. Plotted are the average and standard deviation of $Q^{-1}$ over groups of $10 C D P$ using both the log spectral ratio and centroid frequency methods. The corrected values have less variance and fewer negative $Q$ values. 


\section{APPLICATION TO WAGGONER SITE (REAL DATA)}

With some success in using the method with synthetic and real data, we turned to the 3-D seismic “extension” shoot of the Waggoner Ranch (Figure 62). This dataset was acquired in a rolling patch pattern using an accelerated-weight-drop source rather than dynamite, and suffered from low center frequency and relatively low signal-to-noise ratio. Noise was higher than usual, since part of the acquisition was around the shores of Electra Lake. There are some structural differences between the 2-D Florida dataset and the 3-D data as well. The 2-D line has denser and more uniform offsets within each CDP. The 3-D data has more irregular offsets, and because of the extra dimension offsets that are adjacent in distance may be widely separated in space. To minimize shot-to-shot variance, we considered keying off the shot location rather than CDP. However, there are many fewer shot locations than CDPs, providing low spatial resolution. Also, with the rolling patch acquisition some shot locations are used multiple times with different receiver groups, complicating this approach as well.

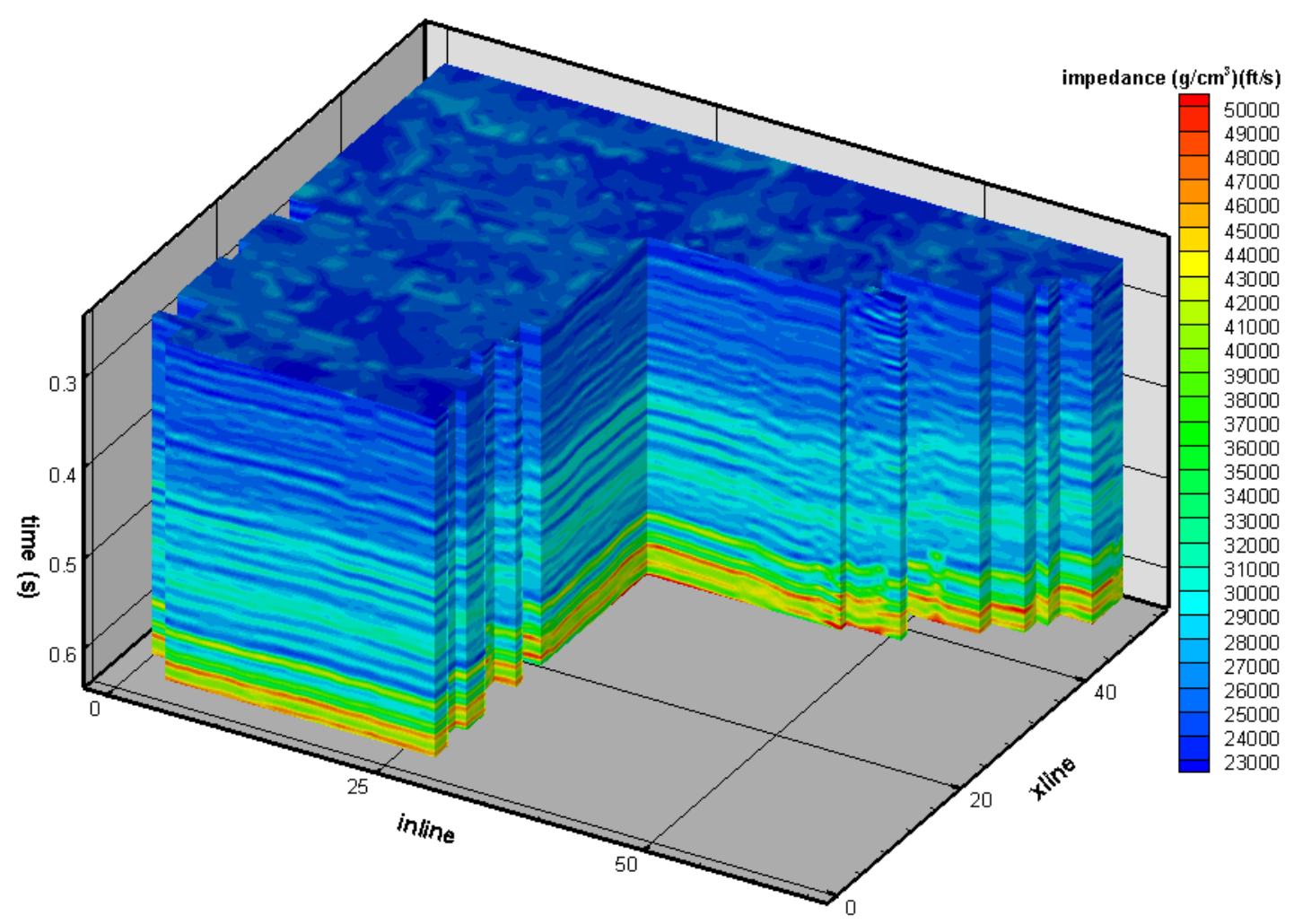

Figure 62. Cutaway view of 3-D extension survey impedance.

We found that, with the low source frequency, we could get consistent data by using only the earliest and latest coherent reflections as reference and target $(0.2-0.4 \mathrm{~s}$ and $0.7-0.9 \mathrm{~s}$, respectively). This corresponds to averaging over depths from roughly 
274 to $1220 \mathrm{~m}$ (900 to $4000 \mathrm{ft}$ ). The best results came from CDP gather data without spectral correction or source deconvolution. The centroid frequency shift method provided smoother results than the spectral ratio method, although both came up with similar averages. Figure 63 shows the computed $Q_{\mathrm{CF}}{ }^{-1}$ based on the target and reference reflections from the Waggoner extension shoot. The average $Q^{-1}$ is around $0.05(Q=20)$, which is reasonable for the finely layered shaley sandstone formations in the area. A few unrealistically high (red) and low (blue) values are spread throughout the data map, particularly along the lake edge which bounds the survey at high inlines. The approximate locations of wells 1,2 , and 3 are marked on the map for reference.

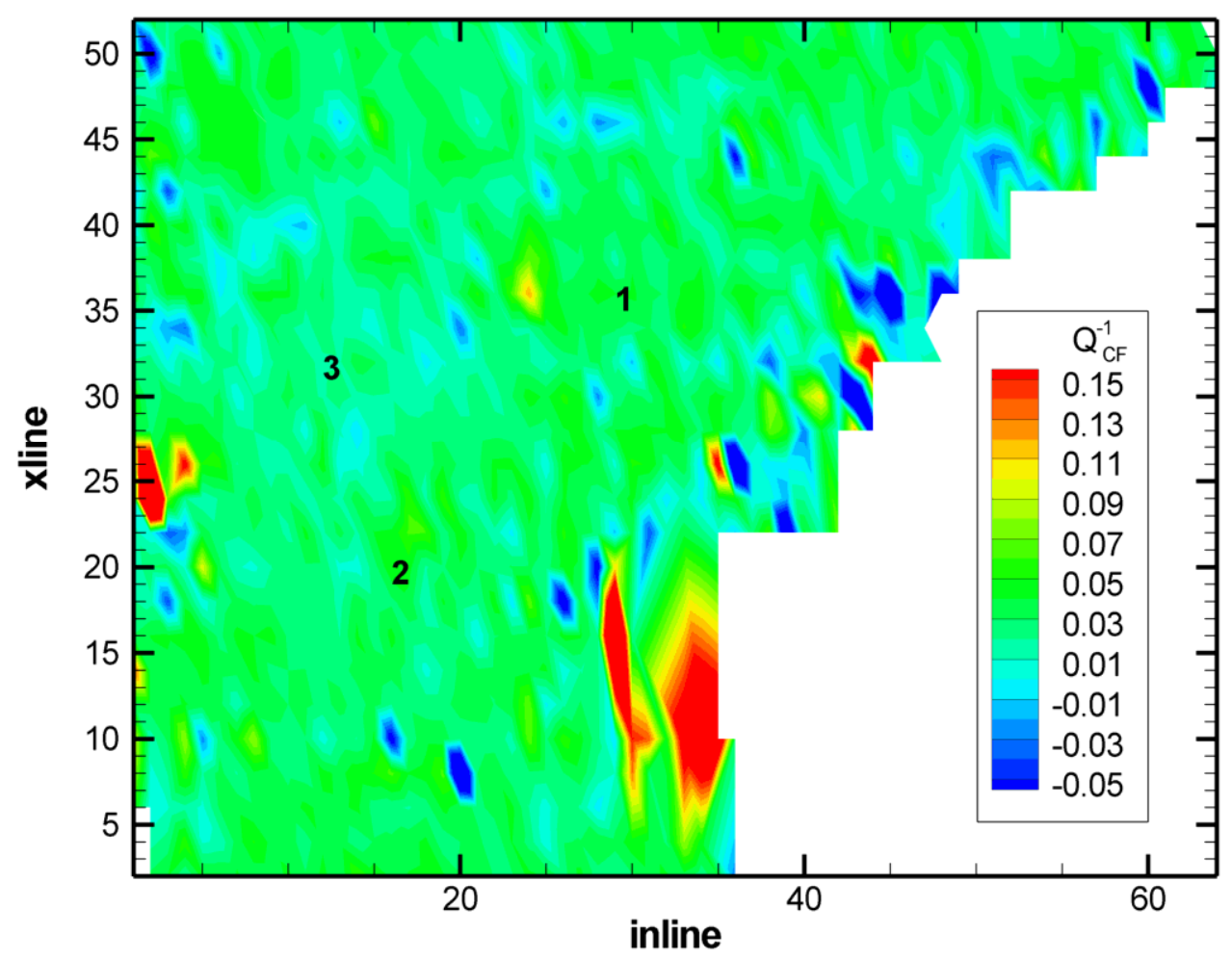

Figure 63. Map of $Q^{-1}$ computed via the centroid frequency $Q V O$ method from the Waggoner extension shoot. The approximate locations of wells 1, 2, and 3 are marked.

A new 3-D seismic survey was conducted adjacent to the extension shoot. This data set was less noisy and contained higher frequencies than the extension shoot. An impedance volume for the new survey was completed based on logs from well \#5 (Figure 64). This was the only well where the synthetic data showed a respectable match to the real data in the STRATA program $\left(\mathrm{R}^{2}=0.70\right)$. 


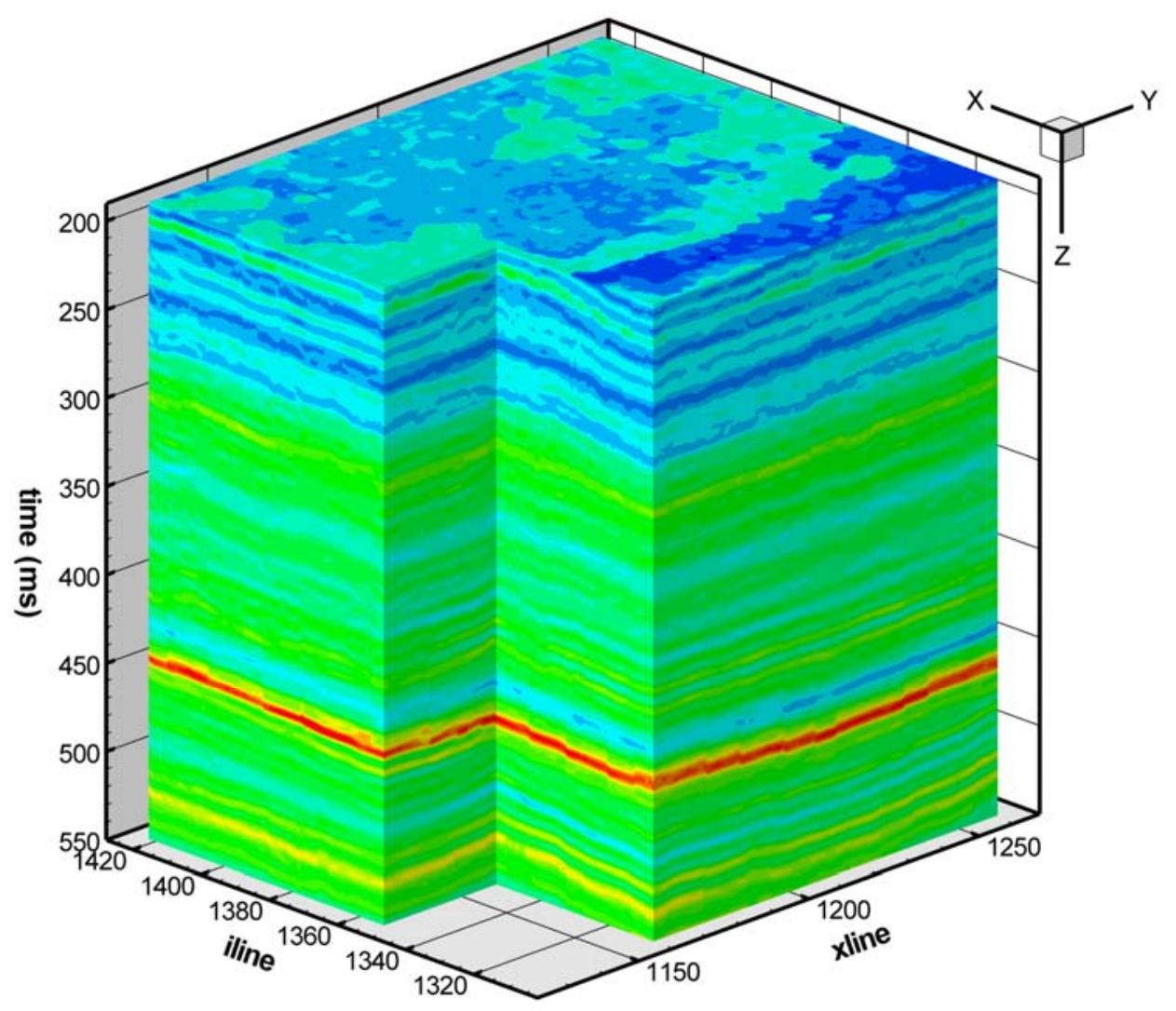

Figure 64. Impedance volume image of Waggoner Ranch 3D seismic data.

We applied the $Q$ processing algorithm to the new 3-D seismic volume from Waggoner, trying to focus more tightly on the reservoir zone. The result (Figure 65) is an average $Q$ from $200 \mathrm{~ms}$ to $600 \mathrm{~ms}$, or roughly from a 274 to $915 \mathrm{~m}$ (900 to $3000 \mathrm{ft}$.) in depth. Resulting $Q$ values are spotty and have only minimal lateral consistency. Better $Q$ values might have been obtainable if we had more (and more accurate) well control. 

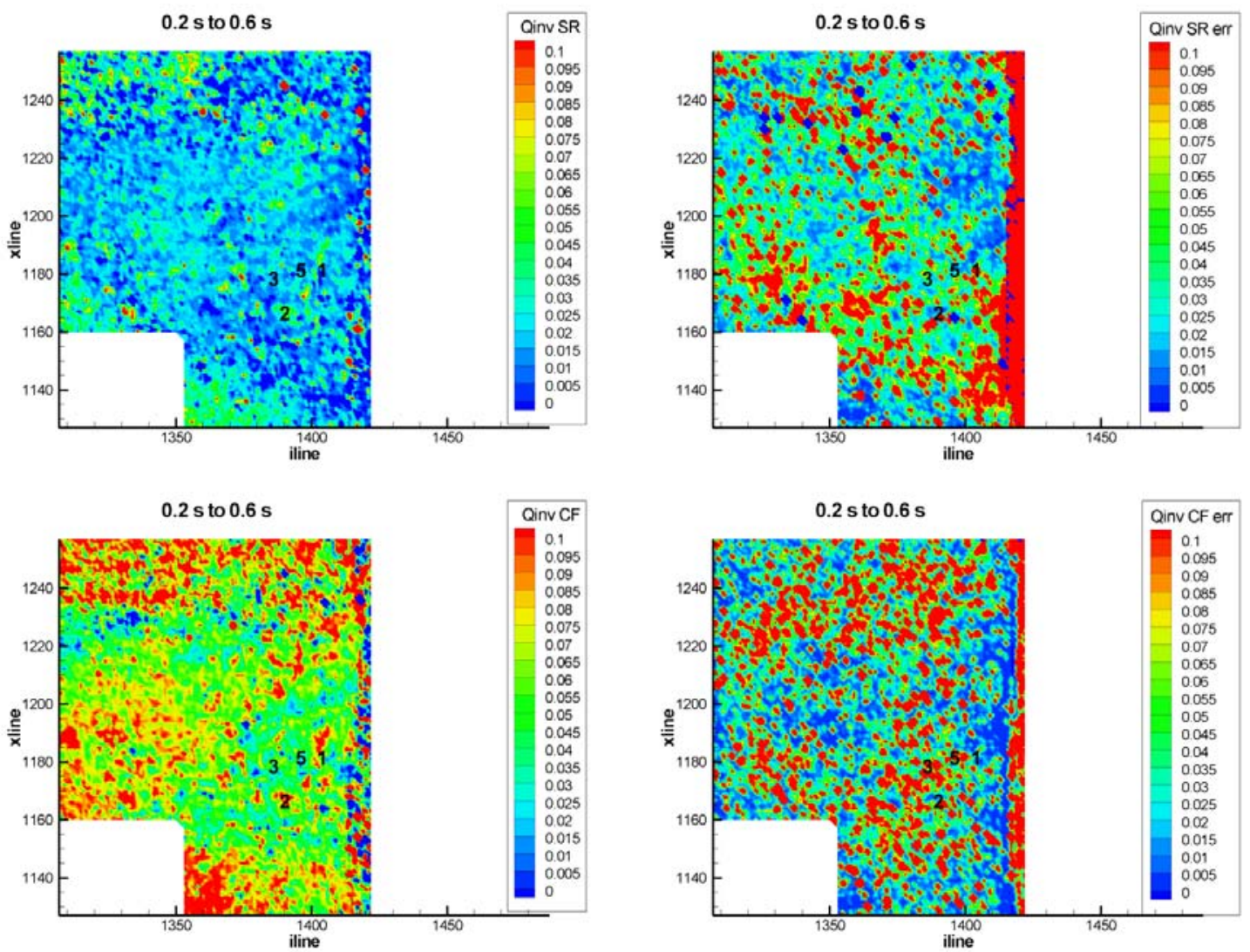

Figure 65. $Q$ values resulting from processing of the Waggoner $3 D$ seismic data. The upper left plot is $Q$ using the spectral ratio method, the upper right plot is the associated uncertainty, the lower left plot is $Q$ using the centroid frequency shift method, and the lower right plot is the associated uncertainty.

From these plots, it is apparent that the spectral ratio (SR, upper left) and centroid frequency (CF, lower left) results agree in trend but not in magnitude: the CF $Q^{-1}$ is about 0.03 higher than the SR result. The SR values are generally in the range of $20<Q<50$, but many areas have $Q$ that seems too high $(Q>100)$. The $C F$ values are generally in the range of $14<Q<25$, but many locations have $Q$ that seems too low $(Q<10)$. In addition, the intrinsic error estimates (upper and lower right) are generally larger than the actual value predicted, implying a low degree of confidence in the results.

We have compared the $Q$ structure with seismic impedance by vertically integrating the seismic impedance from 200 to $550 \mathrm{~ms}$ in order to yield a comparable grid of (x, y) values over a similar time (depth) range. There seems to be no good correlation between the measured $Q$ values and the known impedance structure (Figures 66 and 67). This indicates that either the $Q$ image is of sufficiently poor quality that it is uninterpretable, or that the $Q$ is highly variable at this site and does not correlate with any other known parameters. We also tried additional processing of the $Q$ values to smooth the results into larger bins. This reduces the variability, but still does not produce a consistent and interpretable image of $Q$ at this site. 

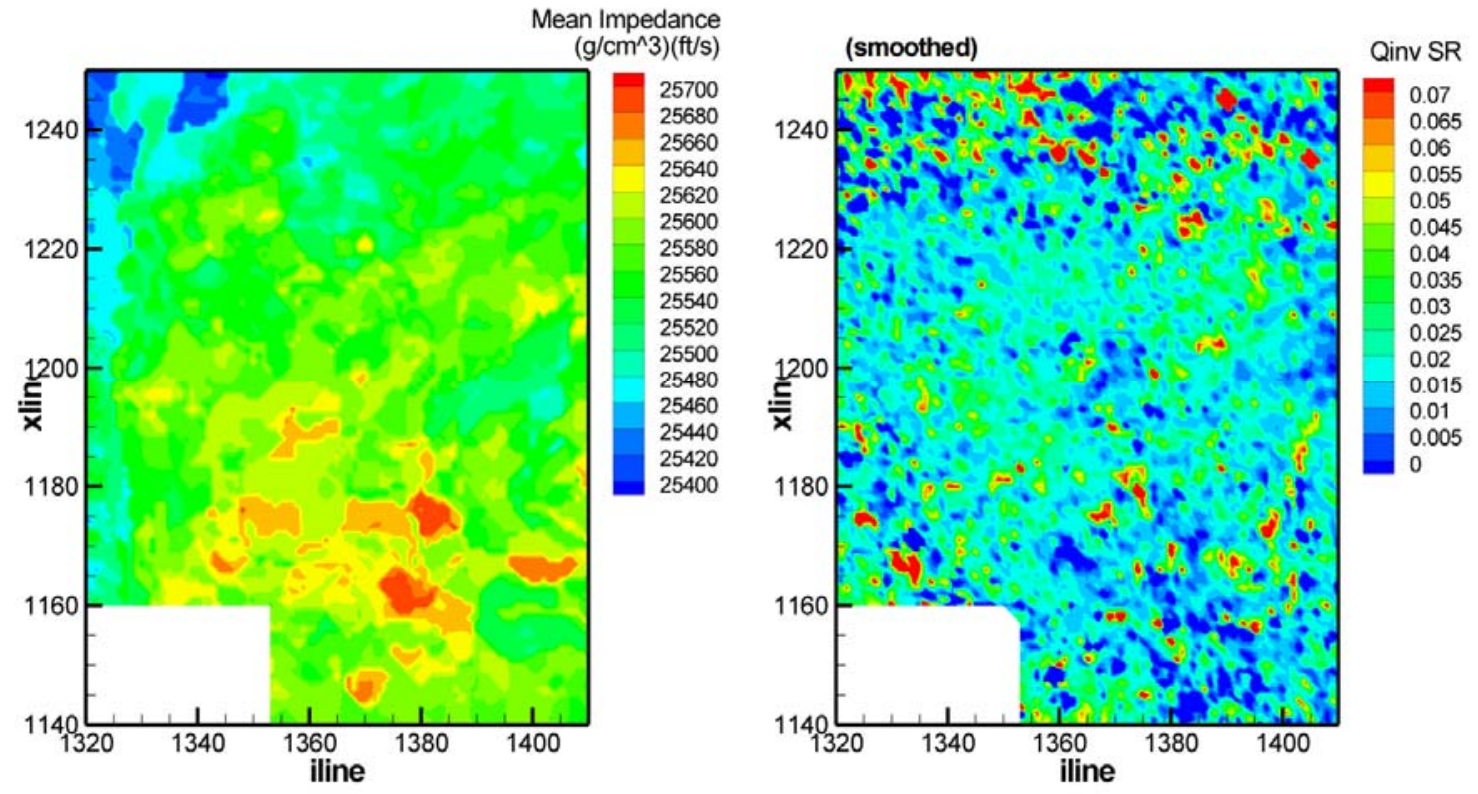

Figure 66. Comparison of mean structural impedance and recovered spectral ratio $Q$ values for comparable time horizons.

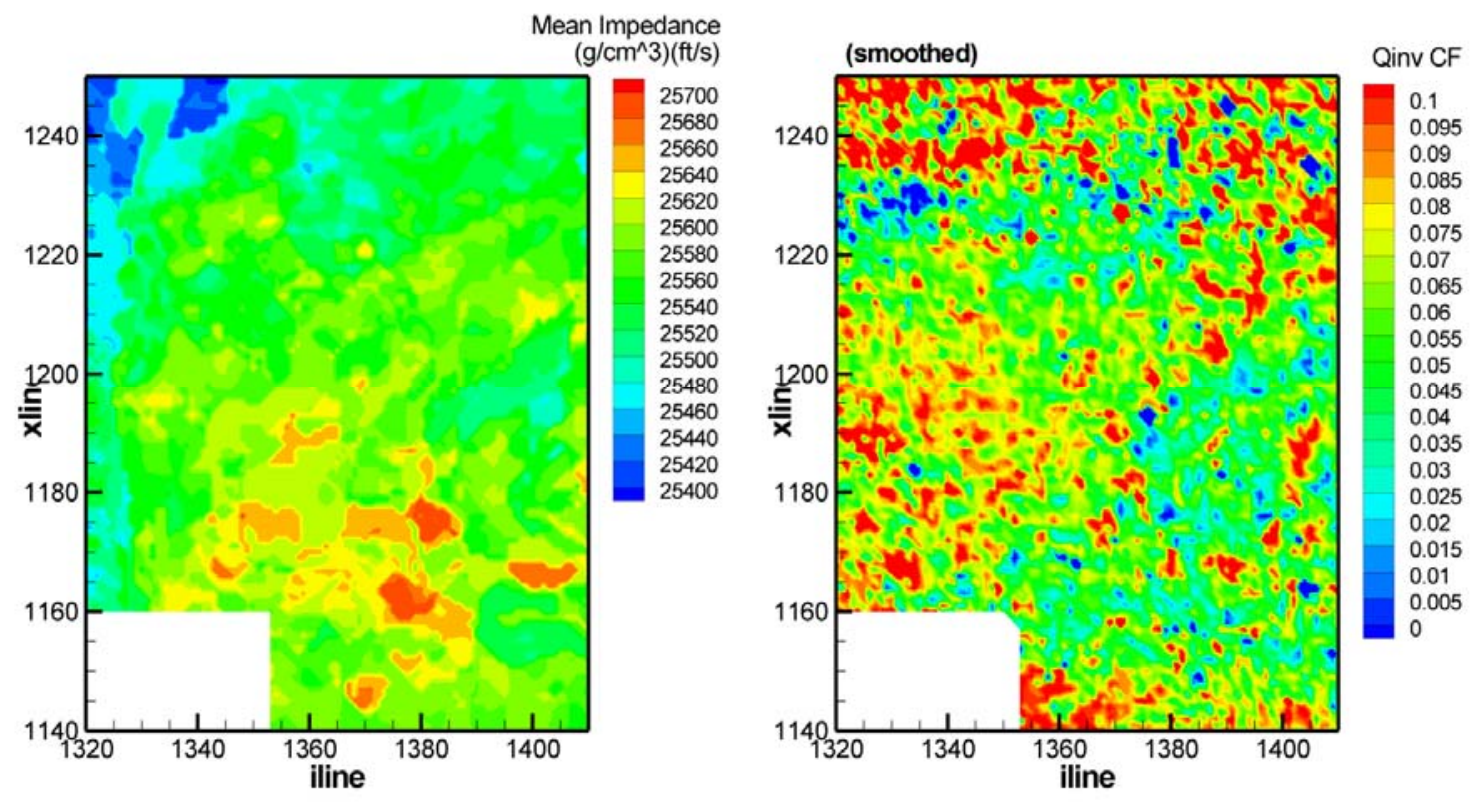

Figure 67. Comparison of mean structural impedance and recovered centroid frequency shift $Q$ values for comparable time horizons.

We attribute the failure to obtain good $Q$ estimates at the Waggoner site to the large number of thin layers at the site. The poor well-log-to-seismic tie prevents us from using the thin-bed correction algorithm with this data set. In addition, we have reduced seismic bandwidth and lower CMP density than we did with the Florida data set. 


\section{EFFECTS OF LATERAL VARIATIONS ON Q MEASUREMENTS}

To quantify the resolution of lateral changes in $Q$ and the effects of lateral changes on the measured $Q$ values, we chose to work with noise-free synthetic data. This allowed us to work with known $Q$ values and specify our vertical and horizontal boundaries precisely. We used a 5-layer, 2-D model with the rock properties shown in Table 10, based loosely on those used in Taner and Treitel (2003). The water content in the sand layer of the model varied laterally from wet sand $\left(\mathrm{S}_{\mathrm{w}}=100 \%\right)$ to fizz sand $\left(\mathrm{S}_{\mathrm{w}}=\right.$ $80 \%)$ to gas sand $\left(\mathrm{S}_{\mathrm{w}}=20 \%\right)$ and back again, as shown in Figure 68. Shear velocities for shale and clean wet sand were computed from P-velocities based on empirical correlations for saturated sands and shales given in Mavko et al. (1998). Shear velocities for partially saturated sands were based on the invariance of the shear modulus with fluid saturation. The numerical model extended for an additional $2000 \mathrm{~m}$ on either side of the primary domain, with the sand layer continuing fully saturated.

Table 10. Rock Properties for 5-Layer, 2-D Model

\begin{tabular}{|l|l|l|l|l|l|}
\hline \multicolumn{1}{|c|}{ Layer } & Vp $(\mathrm{m} / \mathrm{s})$ & Vs $(\mathrm{m} / \mathrm{s})$ & $Q$ & Density $\left(\mathrm{g} / \mathrm{cm}^{3}\right)$ & Thickness $(\mathrm{m})$ \\
\hline Shale 1 & 3300 & 1672 & 46 & 2.30 & 400 \\
\hline Shale 2 & 3500 & 1827 & 53 & 2.44 & 400 \\
\hline Wet sand $(\mathrm{Sw}=100 \%)$, & 3210 & 1725 & 33 & 2.29 & \\
Fizz sand (Sw=80\%), & 2990 & 1737 & 15 & 2.26 & \multirow{2}{*}{250} \\
\cline { 2 - 6 } Gas sand (Sw=20\%) & 3020 & 1776 & 25 & 2.15 & \\
\hline Shale 3 & 3500 & 1827 & 40 & 2.22 & 450 \\
\hline Shale 4 & 3700 & 1981 & elastic & 2.53 & \\
\hline
\end{tabular}

To compute the synthetic seismograms, we used a 2-D finite-difference timedomain viscoelastic model. This model uses a staggered grid, with normal stresses at cell centers, displacements and densities defined at cell faces, and shear stresses at the cell

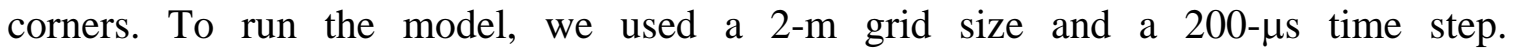
Viscoelasticity was implemented as a time-domain relaxation phenomenon, using a method similar to that of Carcione (1995). The numerical model edges used the absorbing boundary conditions of Higdon (1991). The source was a Ricker wavelet with a peak frequency of $75 \mathrm{~Hz}$. Receivers were placed every $20 \mathrm{~m}$ from the source, out to a maximum offset of $1000 \mathrm{~m}$ (3280 ft.) on both sides. In simulating a 2-D seismic line survey, we moved the shot point in 100-m (328-ft.) increments. Furthermore, since the model was symmetric across $x=2500 \mathrm{~m}(8200 \mathrm{ft}$.), we were able to exploit symmetry to cut the required number of shot points in half. Basic seismic processing was performed with the SU (Seismic Un*x) system (Cohen and Stockwell, 2001; Stockwell, 1999). This included muting of direct waves, sorting, NMO correction, and stacking. 


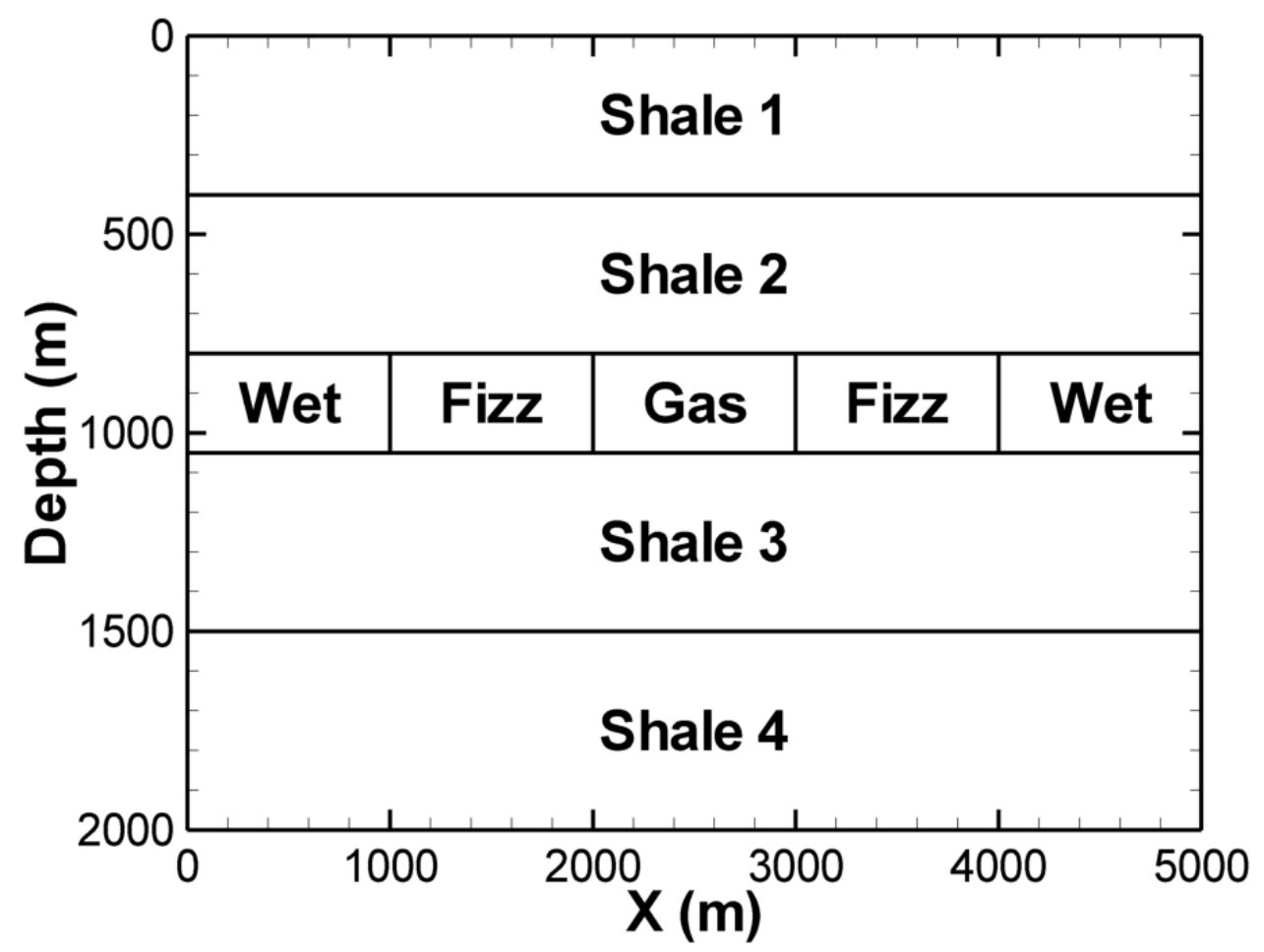

Figure 68. Test geometry. The middle layer consists of wet sand, fizz sand, and gas sand. The rock properties are given in Table 10.

$Q$ estimation was accomplished using the log spectral ratio (LSR) and Q-versusoffset (QVO) method of Dasgupta and Clark (1998). This is one of the few published surface-seismic $Q$ estimation methods to have demonstrated clear success in processing field data. The LSR method is based on seismic energy at higher frequencies attenuating faster than that at lower frequencies. In a frequency-independent $Q$ model, the logarithm of the ratio of spectral amplitudes measured at two points along a ray path is a straight line. The slope of this line is proportional to the product of attenuation and travel time. By measuring the slope of the log spectral ratio from one reflection to another, and by factoring in the known travel-time difference between the two reflections, it is possible to estimate the average $Q$ in the rock between the two reflectors. In the QVO technique, an estimate of $Q$ at zero offset is produced for each CMP by computing the attenuation for every offset in the gather, and extrapolating $Q$ back to zero offset. The attenuation is computed using the LSR method and the zero-offset two-way travel time between the reflections. Since larger offsets involve longer path lengths and travel times, they have a higher apparent attenuation for a given reflector separation. As the difference in path length is proportional to offset-squared (for small offsets), the apparent $Q^{-1}$ values are extrapolated back to zero offset to estimate the true $Q^{-1}$ of the medium between the two reflections. By using traces at multiple offsets, the QVO method not only corrects for the bias toward higher attenuation at long offset, but it can average out some of the random error in each trace to produce a more reliable $Q$ estimate. 


\section{A. Results}

To provide baseline information for the lateral variation tests, we first ran the simulation program with laterally homogeneous sandstone cases (i.e., only wet sand, or only fizz sand, or only gas sand). In the laterally homogeneous cases, only a single shot is needed to acquire all desired data. The results of these tests are summarized in Table 11. These results are presented as both the direct measurement of $Q^{-1}$ from the first reflection to the second, third, and fourth reflections using the QVO method, and as layer-by-layer $Q$ values using layer stripping of the direct measurements. (Layer stripping is a method for computing an interval $Q$ for a specific layer from an average $Q$ over many layers by removing the attenuating effects of higher layers with known $Q$ values, e.g., Dasgupta and Clark, 1998.) Note that the direct estimated attenuation is slightly less than the "true" attenuation in each case, but within about $10 \%$ of the nominal value. This is partly an artifact of trying to simulate constant $Q$ with frequency-dependent attenuation models, and (for lower reflectors) partly due to frequency-dependent transmission coefficients at each interface due to the velocity dispersion that accompanies attenuation. Similarly, although the $Q$ of shale 2 is the same in all three models, the frequencydependence of the reflection coefficient at the top of the sand layer is different in each case, leading to slightly different estimates of $Q$ in shale 2 .

Turning now to the horizontally varying model, the processed and stacked data from the simulated 2-D line is shown in Figure 69. CMPs are spaced every $10 \mathrm{~m}$. The effects of each sand section can be seen in the data. The decrease in velocity in the gassaturated sections shows itself in a slight drop to later times in the reflections at the base of the sand and below. The low velocity and density in gas-charged sections also manifest themselves in increased magnitude of the reflection coefficient, resulting in "bright spots" at the top and base of the sandstone layer. The increased attenuation in these sections is also apparent in the reduced amplitude and frequency of later reflections.

Table 11. True and Estimated $Q$ Values for Laterally Homogeneous Cases

\begin{tabular}{|c|c|c|c|c|c|c|}
\hline & \multicolumn{2}{|c|}{ Water Sand } & \multicolumn{2}{|c|}{ Fizz Sand } & \multicolumn{2}{|c|}{ Gas Sand } \\
\hline & True & Measured & True & Measured & True & Measured \\
\hline \multicolumn{7}{|l|}{ Direct measurement } \\
\hline Reflection $1-2 Q^{-1}$ & 0.0189 & 0.0181 & 0.0189 & 0.0189 & 0.0189 & 0.0180 \\
\hline Reflection $1-3 Q^{-1}$ & 0.0235 & 0.0211 & 0.0381 & 0.0349 & 0.0274 & 0.0256 \\
\hline Reflection $1-4 Q^{-1}$ & 0.0241 & 0.0219 & 0.0329 & 0.0291 & 0.0264 & 0.0246 \\
\hline \multicolumn{7}{|l|}{ Layer stripping } \\
\hline Shale $2 Q$ & 53 & 55 & 53 & 53 & 53 & 56 \\
\hline Sand $Q$ & 33 & 39 & 15 & 17 & 25 & 27 \\
\hline Shale $3 Q$ & 40 & 43 & 40 & 49 & 40 & 44 \\
\hline
\end{tabular}




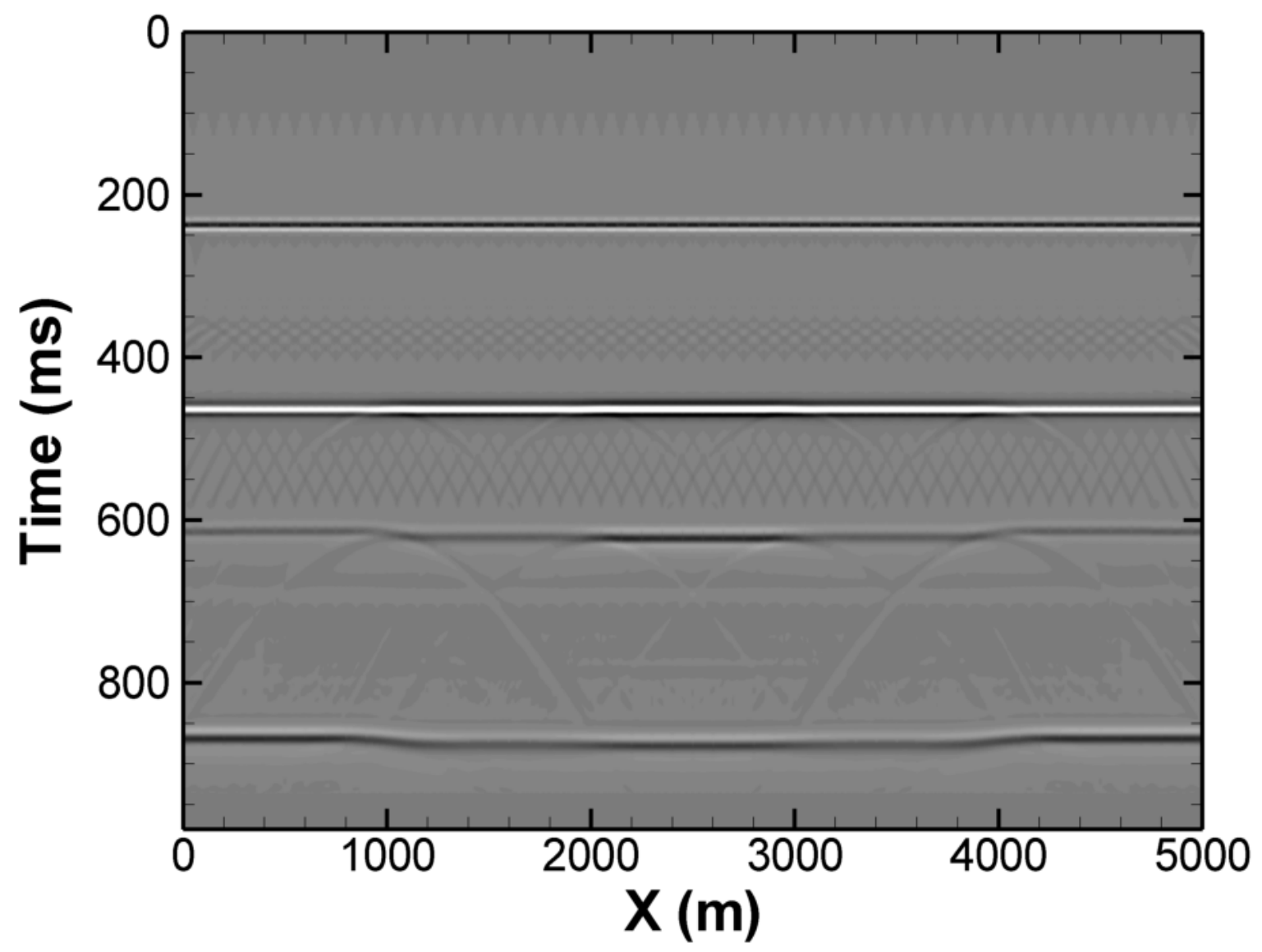

Figure 69. Processed and CMP-stacked data based on model of Table 11.

We estimate $Q$ for each offset of every CMP gather using the log spectral ratio method as discussed above. Figure 70 shows a typical example of log-spectral-ratio data for one offset of three different CMP on the third reflection (base of sand layer). At $x=$ $580 \mathrm{~m}(1640 \mathrm{ft})$, the resulting $Q$ is affected by the water sand; at $1580 \mathrm{~m}, Q$ is affected by the fizz sand; and at $2580 \mathrm{~m}(8462 \mathrm{ft}), Q$ is affected by the gas sand. This plot shows the three distinct slopes associated with the three distinct $Q$ values. The shallowest slope (least attenuation, solid line) is in the water sand, and the steepest slope (highest attenuation, dotted line) is in the fizz sand. At very low and very high frequencies, the signal falls into the background noise and no longer follows the linear trend on the plot. 


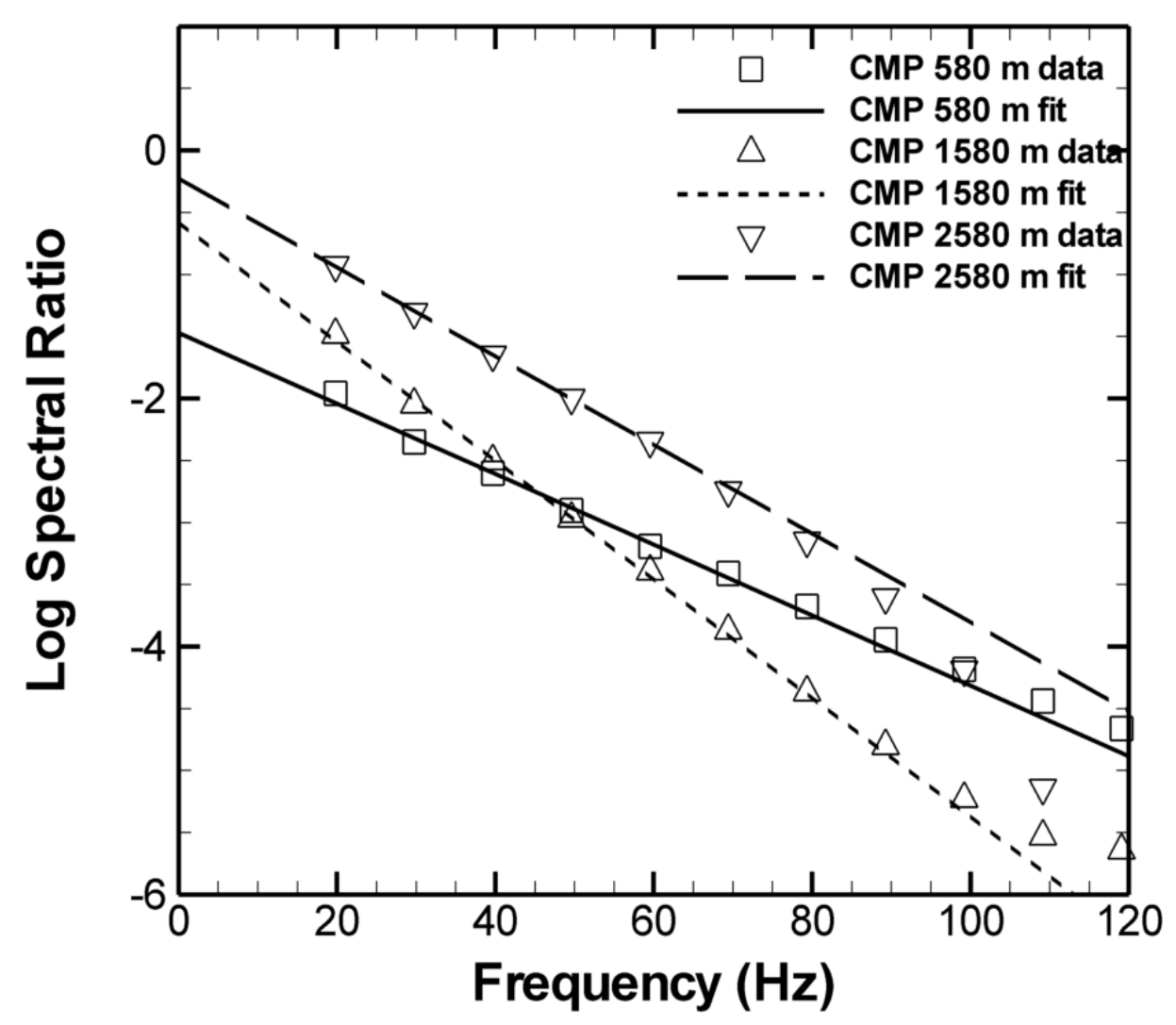

Figure 70. Plot of log-spectral ratios of three traces, from reflections 1 to 3, at different CMP locations. The offset is $640 \mathrm{~m}$ in each case. CMP $580 \mathrm{~m}$ is at the water sand, CMP $1580 \mathrm{~m}$ (5182 ft.) is at the fizz sand, and CMP $2580 \mathrm{~m}$ (8462 ft.) is at the gas sand.

We then apply the QVO method, extrapolating $Q^{-1}$ to zero offset for each CMP, every $10 \mathrm{~m}$ along the line. This provides $Q^{-1}$ as a function of lateral extent across the reservoir for each reflection. The resulting data are plotted in Figure 71. The measured $Q^{-1}$ between the first and second reflections shows little lateral variation, and has a value very close to the nominal level of attenuation. This is as expected, since shale 2 is uniform. The small oscillations in $Q^{-1}$ are due to different offsets being available for different CMP, and have a spatial period of $100 \mathrm{~m}$ (the shot spacing). 

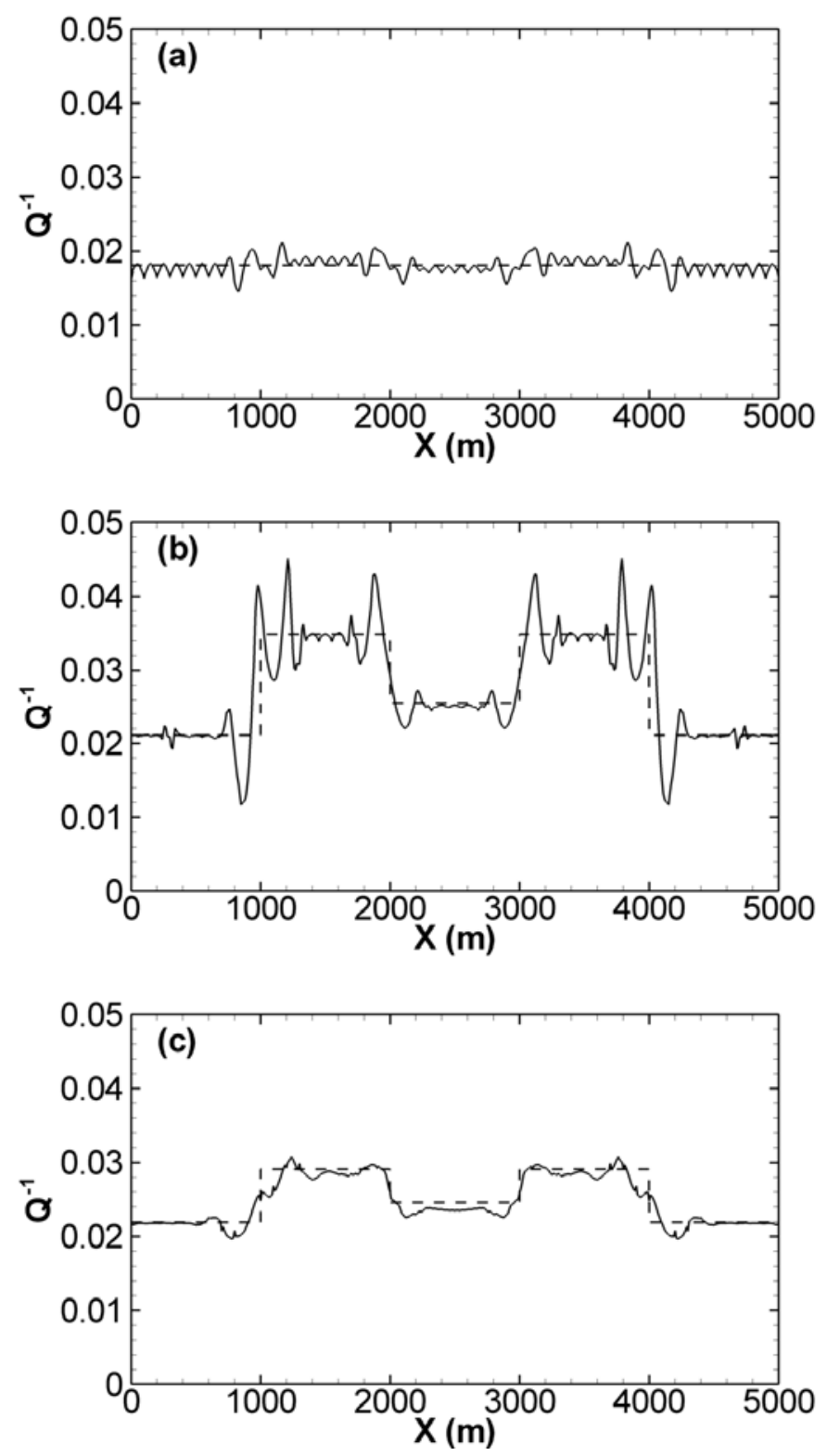

Figure 71. Lateral variation of apparent $Q^{-1}$ : (a) from base of shale 1 to base of shale 2 (top sand), (b) from base of shale 1 to base of sand layer, and (c) from base of shale 1 to base of shale 3. Solid line is measured $Q^{-1}$, and dashed line is $Q^{-1}$ from laterally homogeneous case.

There are additional excursions at the saturation boundaries of the sand unit underlying reflector 2. The measured $Q^{-1}$ between the first and third reflections (top shale 2 to base sand) mostly follows the nominal trend of low, high, and medium attenuation in the various sand types. However, there is quite a bit of variability in attenuation measured between these reflections, especially near the boundaries between different sand units. The attenuation appears enhanced in the fizz sand unit near the edges, suppressed in the water and gas sands near the boundaries, and oscillates 
somewhat as the CMP moves away from the edge. The measured $Q^{-1}$ between the first and fourth reflections (top shale 2 to base shale 3) again follows the same trend seen in the sand units, but with much less variability in the data. Here, the measured attenuation closely matches the expected values, but with some smoothing and some oscillations for CMP below the sand block boundaries.

The oscillatory nature of the variability near the saturation boundaries in and below the sand units suggests that this is the result of wave interference. Snapshots of the vertical motion wavefield (Figure 72) show that diffracted waves are generated at the corners of the saturation blocks. Trorey (1970) discusses how diffraction occurs whenever a reflecting boundary comes to an edge, or when the reflection coefficient changes along a boundary. Diffractions in our model are thus produced by the change in reflection coefficient along the top and base sand, as well as from the edges of the vertical boundaries that divide the saturation units in the sand layer. Trorey (1977) and Berryhill (1977) show that in CMP gathers, diffractions at all offsets have the same polarity and waveform, and similar amplitudes. If the diffraction results from a change in reflection coefficient along a planar boundary, the peak magnitude of the diffracted wave is one-half of the difference in reflection coefficient across the edge. This peak in diffracted amplitude occurs where the diffraction meets the reflection, which maximizes wave interference. Assuming the reflection coefficient does not change sign at the edge, the diffracted wave has polarization opposite to the reflection under the stronger reflection and the same polarization as the reflection under the weaker reflection. Note that diffractions also propagate in the forward direction and are tangent to the direct wave as well as the reflected wave.

Furthermore, reflected $\mathrm{P}$ and both reflected and transmitted converted P-S waves are generated by the direct wave at the vertical boundary between saturation blocks. Since the direct wave is generally arriving at a steep angle to the vertical interface, mode conversion is potentially strong and both the transmitted and reflected waves continue downward. When the direct P-wave reaches the base of the sand layer, it is closely followed by the reflected P-wave from the vertical boundary, the diffracted P-wave from the upper block corner, as well as reflected or transmitted shear converted waves. The shear waves, which arrive at a relatively steep angle to the flat interface, produce in turn converted P (P-S-P) wave reflections. The result is that the bottom of the sand layer produces a direct reflection, a direct diffraction, a reflected diffraction, and multiple reflected $\mathrm{P}$ and PSP converted waves. These entire upward-propagating waves become tangent to each other at the CMP of the block boundary. In Figure 72, we can see that there is strong interference in reflections 2, 3, and 4 at $x=1000 \mathrm{~m}$ (near the sand boundary). 

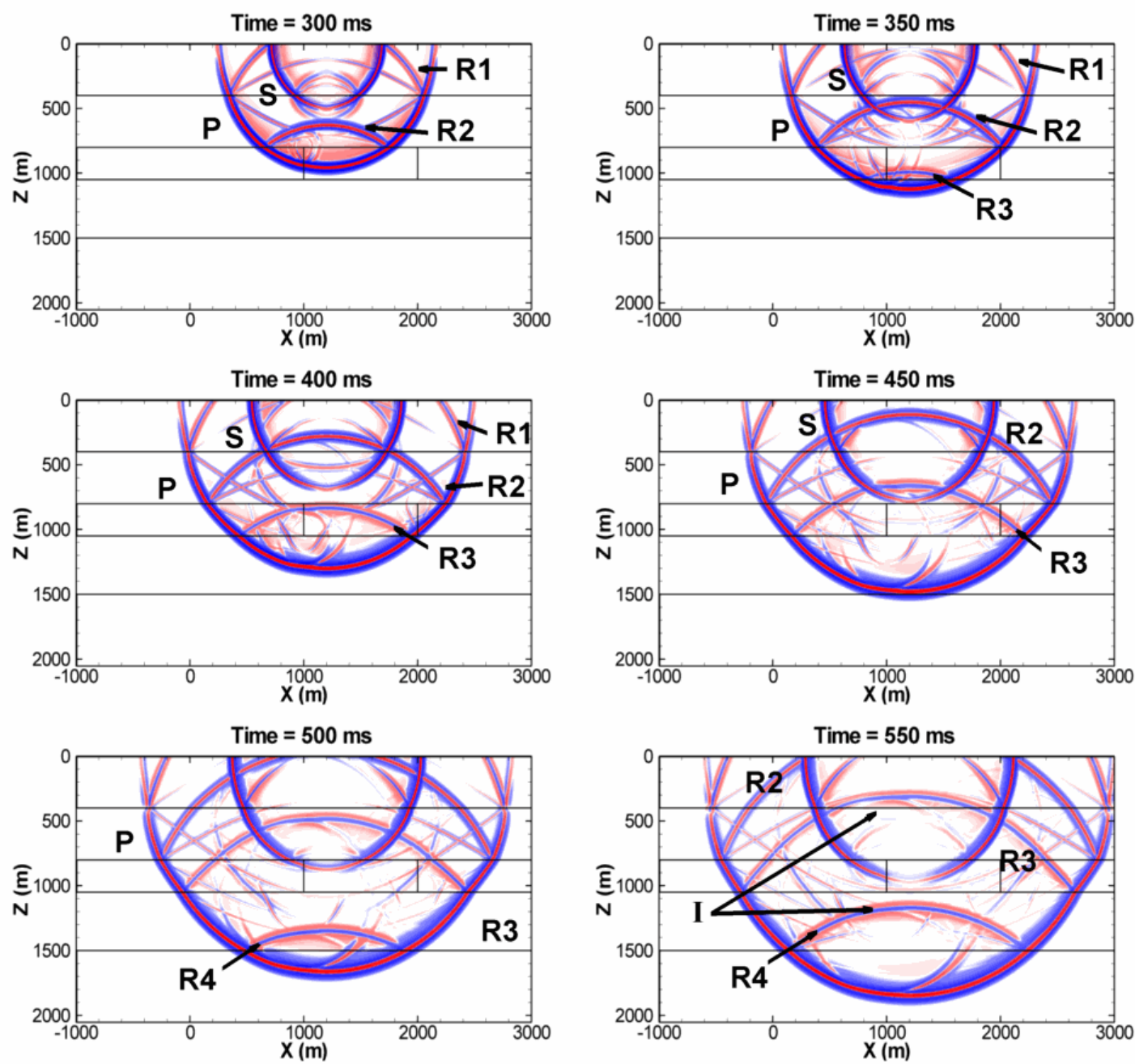

Figure 72. Snapshots of vertical particle velocity at different times, with the source at $x=1200 \mathrm{~m}$ (3936 ft). Red indicates positive vertical velocity, and blue indicates negative vertical velocity. The direct $P$-wave is marked ' $P$,' the direct $S$-wave is marked ' $S$,' and the primary reflections are marked $R 1-R 4$. Locations in the final frame where diffractions and other waves interfere with $R 3$ and $R 4$ are marked as ' $I$.' 
Thus, we conclude that the oscillatory changes in measured attenuation near the lateral boundaries are attributable to interference between the reflected direct waves and the diffracted or multiply reflected waves. These waves decrease in amplitude and move away from the direct reflection as the CMP moves away from the edge. This results in a tuning pattern in which high and low frequencies are alternately enhanced and suppressed with decreasing amplitude away from the boundary. The Q-measurement algorithm depends on the ratio of high to low frequencies, and thus reports incorrect values of $Q$. Since the polarization of the diffracted wave (and sometimes the P-S converted waves) reverses across the horizontal boundary, the pattern of constructive and destructive interference between these waves and the main reflection also reverses. This then has the effect of reversing the sequence of enhanced and suppressed high and low frequencies on the other side of the boundary. These polarity reversals are thus the reason why attenuation is enhanced in the low-Q, low-impedance fizz sand and suppressed in the higher- $Q$, higher-impedance water and gas sands at CMP adjacent to the boundary. Since diffractions are consistent in time and amplitude in NMO-corrected CMP gathers, the tuning effect does not depend on offset and the QVO method reports only the distorted $Q$ value for a given CMP. Figure 73 shows that the $Q$ values for CMP with wave interference are consistently too high or too low regardless of offset.

In summary, the small deviations in measured $Q^{-1}$ at the top sand reflection are due to the interfering effects of diffractions produced at the saturation block boundaries. The larger oscillations in measured $Q^{-1}$ at the base sand reflection are a result of interference from the effects of diffractions at the lower corners of the saturation block boundaries and of reflections of downward-propagating diffractions, reflections, and converted waves from the vertical interface. These downward-propagating waves continue into shale 3, producing somewhat reduced and diffused tuning distortions in measured attenuation at this reflector for CMP below the sand boundaries. As these locally-generated secondary waves travel away from the vertical boundary that is their source, they experience greater geometric spreading loss than the direct wave traveling from the surface, so their effect on the final reflection is much weaker.

At this lowest reflection, the oscillations are sufficiently small in amplitude that we can see some effect of smoothing of the measured $Q^{-1}$ values in the background trend. The smoothing length appears comparable to the size of the first Fresnel zone $(\sqrt{\lambda d / 2})$, where $\lambda$ is the nominal wavelength and $d$ is the depth to the target of interest), which is $180 \mathrm{~m}$ at this depth. However, the smoothing effect is not created at the reflector (as it is in Fresnel smoothing of reflectivity), but rather by different legs of the propagating wave traveling through media with varying attenuation constants. It is not possible to exactly measure the scale of the smoothing because of the overlying oscillations. We attempted to remove the oscillations by running a case in which all sand media had the same densities and $P$ - and $S$-velocities, but different $Q$ values. However, we found that because of the velocity dispersion that accompanies attenuation, there is always some degree of diffraction and reflection at the boundaries separating different $Q$ values. Thus, we were not able to produce a model with laterally varying $Q$ that did not have oscillations in the measured $Q$ values. 

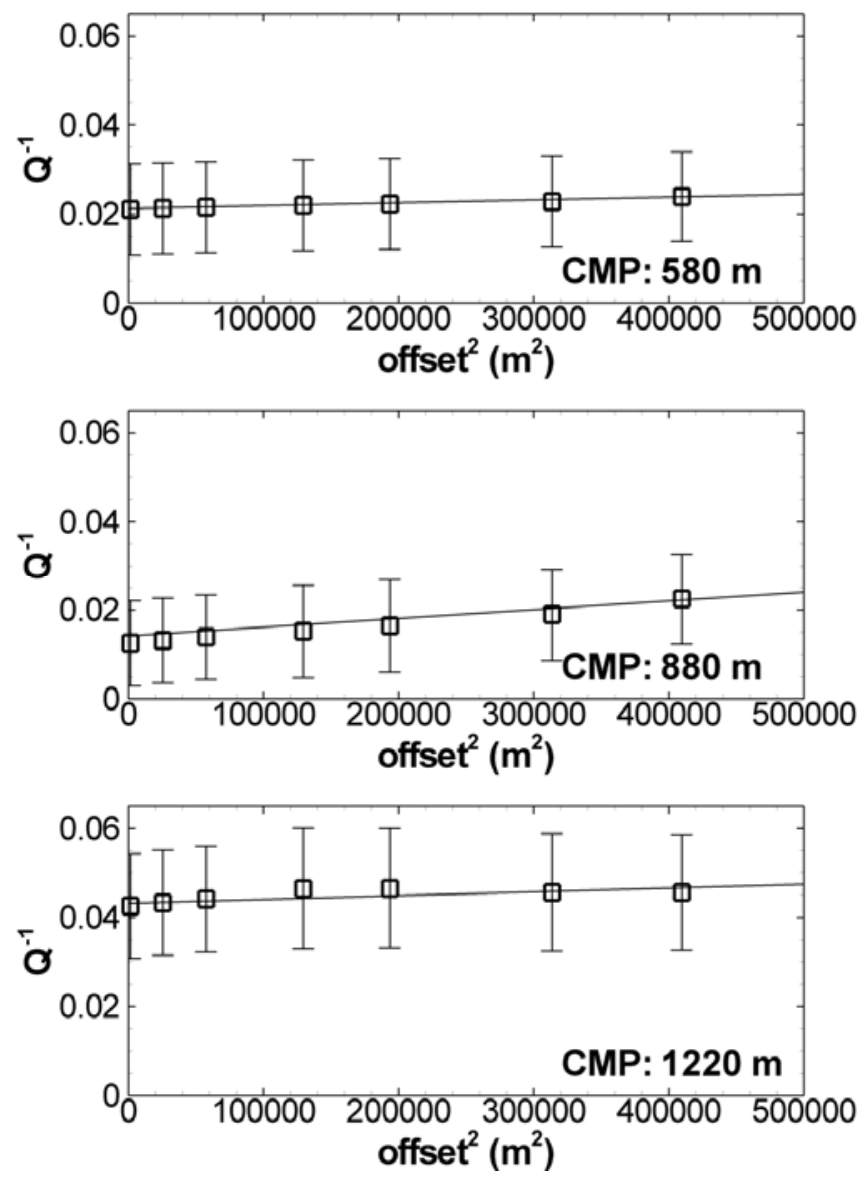

Figure 73. $Q^{-1}$ versus offset-squared for three CMP gathers at the base sand reflector, showing normal, suppressed, and enhanced attenuation. At CMP $580 \mathrm{~m}$, the measured $Q^{-1}$ behaves normally for all offsets. At CMP $880 \mathrm{~m}$ (2886 ft.), the measured $Q^{-1}$ is low at all offsets. At CMP $1220 \mathrm{~m}$ (4001 ft.), the measured $Q^{-1}$ is high for all offsets. 


\section{TECHNOLOGY TRANSFER}

A significant part of this project is technology transfer. We made nine presentations at professional conferences and wrote a total of eight papers and one book chapter. Two of the papers are in press and will be published in 2006.

We presented the Q-log algorithm and applications at the SEG Development and Production Forum in Austin, Texas, in May 2005. The presentation attracted significant interest from the attendees and, at the request of the SEG delegates, a powerpoint presentation of the paper was placed on the Institute internet site. The presentation can be obtained directly from the link:

http://Www.swri.org/4org/d15/elecsys/resgeo/ppt/Algorithm.pps

We presented a second application of the $Q$-log algorithm at the SEG International Conference in Houston, Texas, in November 2005. This presentation attracted significant interest from delegates as well. It can be obtained directly from the link:

http://wWw.swri.org/4org/d15/elecsys/resgeo/ppt/attenuation.pps

\section{A. Presentations}

Hackert, C.L. and Parra, J.O. (2004) "Improving Q estimates from seismic reflection data using well-log based spectral correction,” presented at SEG $74^{\text {th }}$ International Meeting, October 10-14, Denver, Colorado.

Parra, J.O., and Hackert, C.L. (2003) "High resolution P-wave surface seismic profiling to delineate flow units in carbonate aquifers of south Florida: a feasibility study," presented at the Society of Exploration Geophysicists (SEG) 73rd Annual International Meeting, October 26-30, Dallas, TX. Expanded Abstracts, paper RCT P1.8.

Parra, J., Hackert, C.L., Green, R., and Pride, S. (2003) "A double-porosity poroelastic model to relate P-wave attenuation to fluid flow in vuggy carbonate rock," presented at GeoProc 2003, October 13-15, Stockholm, Sweden, paper published in GeoProc 2003, Stephansson et al., (eds.), pp. 481-486.

Parra, J.O., Hackert, C.L., Bennett, M., and Collier, H.A. (2003) "High-resolution acoustic and seismic investigation of carbonate rock properties," presented at the 44th Annual SPWLA Symposium, June 23-25, Galveston, TX, paper NN in SPWLA 44th Annual Logging Symposium Transactions.

Parra, J.O., Hackert, C.L., and Xu, P.C. (2004) "Q logs for lithology and pore structure discrimination,” presented at the SEG $74^{\text {th }}$ International Meeting, October 10-15, Denver, CO. Expanded Abstracts, 1754-1757. 
Kazatchenko, E., Markov, M., Mousatov, A, and Parra, J.O. (2004) "Application of petrophysical inversion of well logs for pore-system characterization of a south Florida aquifer,” presented at the SEG $74^{\text {th }}$ International Meeting, October 10-15, Denver, CO. Expanded Abstracts.

Parra, J.O., and Xu, P.-C. (2005) "An algorithm to extract $Q$ from sonic logs and its application to a double porosity carbonate aquifer," presented at the SEG Development and Production Forum, Austin, Texas, May 15-19, 2005.

Parra, J.O., Hackert, C.L., and Xu, P.-C. (2005) "Attenuation analysis and modeling of acoustic waveforms in a borehole intercepted by a sand shale sequence," presented at the SEG $75^{\text {th }}$ Annual International Mtg., November 6-11, Houston, TX. Expanded Abstracts, paper BG 1.1.

Korneev, V., Parra, J., and Bakulin, A. (2005) "Tube-wave effects in crosswell seismic data at the Stratton Gas Field,” presented at the SEG $75^{\text {th }}$ Annual International Mtg., November 6-11, Houston, TX. Expanded Abstracts, paper BG 1.4.

Parra, J.O. (2004) "Method for extracting quality factor Q from full waveform sonic logs,” presented at the Instituto Mexicano del Petróleo, Mexico City, Mexico, May 2004.

\section{B. Publications}

Parra, J.O., Hackert, C.L., Bennett, M., and Collier, H.A. (2003) "Permeability and porosity images based on NMR, sonic, and seismic reflectivity: Application to a carbonate aquifer,” The Leading Edge 22, 1102-1108 (DOI 10.1190/1.1634914).

Hackert, C.L., and Parra, J.O. (2003) "Estimating scattering attenuation from vugs or karsts,” Geophysics 68, 1182-1188 (DOI 10.1190/1.1598111).

Hackert, C.L., and Parra, J.O. (2004) "Improving $Q$ estimates from seismic reflection data using well-log-based localized spectral correction,” Geophysics 69, 521-1529.

Parra, J.O., Hackert, C.L., and Pride, S. (2004) "Double-porosity poroelastic model to relate P-wave attenuation to fluid flow in vuggy carbonate rock," in Coupled ThermoHydro-Mechanical-Chemical Processes in Geo-Systems, 2, O. Stephansson, J. Hudson, and L. Jing, eds., Elsevier, 483-488.

Parra, J.O., Hackert, C.L., Bennett, M., Jervis, M. and Collier, H. (2005) “An integrated approach based on NMR/acoustic techniques to map permeability in carbonate aquifers: from the pore to field scales," in Near-Surface Geophysics, SEG, Investigation in Geophysics Series No. 13, D.K. Butler, ed., 473-489.

Parra, J.O., and Hackert, C.L. (2006) "Modeling and interpretation of $Q$ logs in carbonate rock using a double-porosity model and well logs,” Journal of Applied Geophysics , 58, 253-262.. 
E. Kazatchenko, Markov, M., Mousatov, A., and Parra, J.O. (2006) "Carbonate microstructure determination by inversion of acoustic and electrical data: Application to a south Florida aquifer,” Journal of Applied Geophysics (in press).

Parra, J.O., Hackert, C.L., and Bennett, M.W. (2006) "Permeability and porosity images based on P-wave surface seismic data: Application to a south Florida aquifer," Water Resources Research, 42, W02415 (DOI: 10.1029/2005WR004114).

Parra, J.O., Hackert, C.L., and Xu P.-.C, (2006) Attenuation analysis of acoustic waveforms in a borehole intercepted by a sand-shale sequence reservoir, The Leading Edge, February, 187-193. 


\section{GENERAL CONCLUSIONS}

In this report we present the implementation and application of two methods for estimating $Q$; one for extracting $Q$ from full waveform sonic data $(Q \log )$ and one for estimating $Q$ from surface seismic data. The $Q \log$ algorithm automatically corrects the sonic data for scattering, geometrical spreading, and borehole effects. This algorithm has been tested and verified with synthetic data. In general the algorithm gives excellent results when the receivers are away from an interface. When the algorithm crosses a boundary, it becomes numerically unstable. To handle this problem we corrected the apparent attenuation for coupling between the borehole and the layers. We suggest development of a boundary value problem for a point source in a fluid-filled borehole surrounded by a multilayered earth. This solution will allow to the interpreter to correct the attenuation for coupling effects at any position of the receivers at velocity boundaries.

Despite the need to correct the attenuation near an interface, application of the $Q$ log algorithm to real sonic data proved quite useful in detecting fluids in an oil reservoir and characterizing the pore structure in a carbonate aquifer. In the oil reservoir, the $Q \log$ detected a zone that contained oil and water, and it helped describe the rock physical properties of the formation surrounding the borehole. We showed that the $Q \log$ can indeed explain the attributes of the microseismogram, and our results demonstrate that $Q$ is an important log that, when integrated with other well logs, can discriminate between anomalies associated with lithology and those associated with oil and gas saturation.

This study provided original and useful technology for interpreting attenuation from sonic data. We implemented a double porosity model to relate pore structure to attenuation. The effective double-porosity model was successfully adapted to the dynamic poroelastic wave equation to simulate P-wave and slow wave attenuation and spectral responses due to poroelastic fluid flow in vuggy carbonate rocks. We generalized the model to interpret the attenuation logs in carbonate reservoirs and applied it to interpret data from south Florida. We showed that, in permeable carbonate rock formations, pore structure can be related to fluid flow between the pore matrix and the vugs. In particular, permeability indirectly influences attenuation through the statistical correlation of attenuation and pore structure.

The attenuation work in south Florida resulted in the complete characterization of a single borehole. Knowledge of lithology and rock physical properties at the borehole and crosswell scales allowed us to evaluate the use of surface seismic to map flow units. We produced permeability and porosity images at the field scale using a single well, and we concluded that surface reflection seismic is a practical and economical method for mapping flow units in carbonate reservoirs, assuming that there is not much lateral variation in the geology. Reflected wavelets were altered in the time and frequency domains by the presence of thin beds near the primary reflector and by lateral variations in seismic properties at the primary reflector or higher strata; these lateral variations made it difficult to extract reliable $Q$ information from the seismic data.

We also successfully demonstrated the use of a well-log-based spectral correction method for improving $Q$ estimation with QVO analysis of surface seismic data. In this method, the spectrum of the reflection is divided by the spectrum of the local reflectivity stickogram before $Q$ is computed. An inherent assumption is that the observed reflections consist of primaries only (multiple-free). We tested the method using synthetic 
seismograms, and then applied it to real data. The $Q$ values computed using the corrected spectra had less variance and fewer negative values than those computed with uncorrected spectra. We found an average $Q$ value near 33 for sediments from roughly $170 \mathrm{~m}$ to $600 \mathrm{~m}$ deep at the south Florida test site. This value generally held stable along the length of the seismic line, using both the line spectral ratio and central frequency methods for $Q$ extraction.

We also applied the $Q$ extraction method to two surface seismic surveys from the Waggoner site, recovering an average $Q$ of 20-50 for the field as a whole, with the LSR and CF methods each producing different results. There was a high degree of variability in the recovered $Q$ values, including many unreasonably high values of $Q$ and many unphysical negative values of $Q$. We were unable to find a consistent trend of $Q$ versus inline/crossline position, or any reasonable correlation of $Q$ versus known structure (based on the impedance volume). We believe this is mostly due to the large number of thin beds at the Waggoner site interfering with the reflection spectra. We were unable to use the spectral correction method here, because of the small number of well logs available and the poor tie between those well logs and the seismic data.

The success of the spectral correction method depends on a good local tie from the well $\log$ to the seismic data. The need for time windows to bracket each reflection group means that $Q$ inversion will be a low-resolution process. The time windows must be long enough (i.e., several periods of the peak frequency of the waveform) so that a Fourier transform produces several frequencies within the bandwidth of the pulse. Otherwise, there will be too few data points for the LSR or CF methods to be reliable. Furthermore, for accurate estimation of travel times, the separation between time windows must be several times their length. For most cases, the recovered $Q$ estimates will be averages over a hundred meters or more of depth. In many formations this may be sufficient to find evidence of gas, fractures, or changes in lithology. If many overlapping $Q$ measurements are taken, there is also the possibility of downscaling the low-resolution $Q$ information to a higher resolution in conjunction with seismic or borehole data.

The lateral-variability modeling study showed that results of $Q$ estimation from surface seismic data should be evaluated cautiously in regions containing faulting, abrupt changes in dip, or rapid horizontal variations in saturation or facies. All of these geological features can produce diffractions and other waves that follow closely behind the direct and reflected waves in some propagation directions. The resulting constructive and destructive interference may hinder accurate spectral estimation in the region of lateral discontinuity. Since the most common methods of $Q$ estimation from reflection data (spectral ratio and centroid-frequency shift) depend on correct spectra, precise $Q$ measurement will be difficult for CMPs that include such lateral inhomogeneities. In addition, if the target of interest is immediately below the region of strong lateral variability, downward-propagating diffractions and reflections from near-vertical interfaces will closely follow the direct wave and can affect spectral measurements in these lower strata as well.

Aside from the interference phenomenon, the horizontal resolution of $Q$ is controlled by the Fresnel zone diameter at the reflector and the paths the traveling wave takes through regions of different seismic attenuation factors. Using the QVO method, the measured $Q$ will be biased toward the short-offset measurements, which minimizes the influence of attenuation from ray paths that travel through volumes far from the CMP 
of interest. Prestack migration may be useful in mitigating the effects of diffractions on CMP near the lateral boundaries, but seems unlikely to help in removing waveform distortions from the downward-propagating waves. Furthermore, any additional manipulation of the surface seismic data must be performed carefully, so as not to introduce any additional spectral distortions through post-processing.

Based on the work reported here, we expect $Q$ estimation from surface seismic to work best in formations with thick, uniform beds; good well control; and no abrupt lateral changes. It will also work best when the seismic source has a large bandwidth, and noise is low. Testing our methods in reservoirs of this type would help to establish the technique. There may also be a benefit in researching the use of wavelet-transform-based rather than Fourier-transform-based spectral estimators. The wavelet-based methods have some potential for reducing the size of the time windows needed to produce spectra, thus providing spectra that are more representative of specific reflectors and transit times. 


\section{REFERENCES}

Adriansyah and G.A. McMechan, 1998, Effects of attenuation and scattering on AVO measurements: Geophysics, 63, 2025-2034.

Aki, K., and P.G. Richards, 1980, Qualitative Seismology, vol. 1: W.H. Freeman and Co.

Bennett, M., P.E. Linton, and E.E. Rectenwald, 2002, Hydrologic investigation of the Floridan aquifer system, western Hillsboro Basin, Palm Beach County, Florida: Technical Publication WS-8, South Florida Water Management District.

Berryhill, J.R., 1977, Diffraction response for nonzero separation of source and receiver: Geophysics, 42, 1158-1176.

Berryman, J.G., and H.F. Wang, 1995, The elastic coefficient of double-porosity models for fluid transport in joined rock: Journal of Geophysical Research, 100, 2461124627.

Biot, M.A., 1962. Mechanics of deformation and acoustic propagation in porous media: Journal of Applied Physics, 33, 1482-1498.

Bouchon, M., 1976, Calculation of complete seismograms for an explosive source in a layered medium: Geophysics, 45, 197-203.

Bouchon, M., 1993, A numerical simulation of the acoustic and elastic wavefields radiated by a source on a fluid-filled borehole embedded in a layered medium: Geophysics, 58(4), 475-481.

Boutin, C., G. Bonnet, and P.Y. Bayard, 1987, Green's functions and associated sources in infinite and stratified poroelastic media: Geophysical Journal of the Royal Astronomical Society, 90, 521-550.

Carcione, J.M., 1993, Seismic modeling in viscoelastic media: Geophysics, 58, 110120.Carcione, J.M., 1995, Constitutive model and wave equations for linear, viscoelastic, anisotropic media: Geophysics, 60, 537-548.

Cardimona, S.J., W.P. Clement, and K. Kadinsky-Cade, 1998, Seismic reflection and ground penetrating radar imaging of a shallow aquifer: Geophysics, 63, 13101317.

Chang, D., H. Vinegar, C. Morriss, and C. Straley, 2001, Effective porosity, producible fluid, and permeability in carbonates from NMR logging: The Log Analyst, 38, 21-43.

Cheng, C.H., M.N. Toksoz, and M.E. Willis, 1982, Determination of in situ attenuation from full waveform acoustic logs: Journal of Geophysical Research, 87, 54775484.

Cohen, J.K., and J.W. Stockwell, Jr., 2001, CWP/SU: Seismic Un*x Release No. 35: A free package for seismic research and processing, Center for Wave Phenomena, Colorado School of Mines.

Collino, F., and C. Tsogka, 2001, Application of the perfectly matched absorbing layer model to the linear elastodynamic problem in anisotropic heterogeneous media: Geophysics, 66, 294-307.

Dasgupta, R., and R.A. Clark, 1998, Estimation of $Q$ from surface seismic reflection data: Geophysics, 63, 2120-2128.

Datta-Gupta, A., G. Xue, and S.H. Lee, 1997, Non-parametric transformations for data correlation and integration: from theory to practice: Proceedings, $4^{\text {th }}$ International Reservoir Characterization Technical Conference, Houston, Texas, March 2-4. 
Day-Lewis, F.D., J.W. Lane, J.M. Harries, and S.M. Gorelick, 2003, Time-lapse imaging of saline-tracer transport in fractured rock using difference-attenuation radar tomography: Water Resources Research, 39, 1290.

Dvorkin, J., and A. Nur, 1993, Dynamic poroelasticity: A unified model with the squirt and Biot mechanisms: Geophysics, 58, 524-533.

Ganley, D.C., and E.R. Kanasewich, 1980, Measurement of absorption and dispersion from check shot surveys: Journal of Geophysical Research, 85, 5219-5226.

Gurevich, B., V.B. Zyrianov, and S. Lopatnikov, 1997. Seismic attenuation in finely layered porous rocks: Effects of fluid flow and scattering: Geophysics, 62, 319324.

Hackert, C.L., and J.O. Parra, 2000, Analysis of multiscale scattering and poroelastic attenuation in a real sedimentary rock sequence: Journal of the Acoustical Society of America, 107, 3028-3034.

Hackert, C.L., and J.O. Parra, 2004, Improving $Q$ estimates from seismic reflection data using well-log-based localized spectral correction: Geophysics, 69, 1521-1529.

Hauge, P.S., 1981, Measurements of attenuation from vertical seismic profiles: Geophysics, 46, 1548-1558.

Hentz, T.F., and L.F. Brown, Jr., 1991, Wichita Falls-Lawton Sheet, Geologic Atlas of Texas, Bureau of Economic Geology, University of Texas at Austin, GA0038.

Higdon, R.L., 1991, Absorbing boundary conditions for elastic waves: Geophysics, 56, 231-241.

Kazatchenko, E., M. Markov, and A. Mousatov, 2004, Joint modeling of acoustic velocities and electrical conductivity from unified microstructure of rocks: Journal of Geophysical Research, 109, B01202.

Kazatchenko, E., M. Markov, A. Mousatov, and J.O. Parra, 2004, Application of petrophysical inversion of well logs for pore-system characterization of a South Florida Aquifer. Presented at the Society of Exploration Geophysicists (SEG) $74^{\text {th }}$ Annual International Meeting, October 10-15, Denver, CO; published in SEG Expanded Abstracts.

Korneev, V.A., G.M. Goloshubin, T.M. Daley, and D.B. Silin, 2004, Seismic lowfrequency effects in monitoring fluid-saturated reservoirs: Geophysics, 69, 522532.

Mavko, G., and A. Nur, 1975, Melt squirt in aesthenosphere, Journal of Geophysical Research, 80, 1444-1448.

Mavko, G., T. Mukerji, and J. Dvorkin, 1998, The Rock Physics Handbook: Tools for Seismic Analysis in Porous Media: Cambridge University Press, UK.

Neep, J.P., M.S. Sams, M.H. Worthington, and K.A. O’Hara-Dhand, 1996, Measurement of seismic attenuation from high-resolution crosshole data: Geophysics, 61, 11751188.

O’Doherty, R.F. and N.A. Anstey, 1971, Reflections on amplitudes: Geophysical Prospecting, 19, 430-458.

Oldenburg, D.W., T. Scheuer, and S. Levy, 1983, Recovery of the acoustic impedance from reflection seismograms: Geophysics, 48, 1318-1337.

Oppenheim, A.V., and R.W. Schafer, 1989, Discrete-time signal processing: Prentice Hall. 
Paine, J.G., 2003, Determining salinization extent, identifying salinity sources, and estimating chloride mass using surface, borehole, and airborne electromagnetic induction methods: Water Resources Research, 39, 1059.

Parra, J.O., 1991. Analysis of elastic wave propagation in stratified fluid-filled porous media for interwell systemic applications, Journal of the Acoustic Society of America, 90: pp. 2557-2575.

Parra, J.O., and C.L. Hackert, 2001, A poroelastic model with azimuthal anisotropy to analyze flow unit responses at the Siberia Ridge Field, Greater Green River Basin: Petrophysics, 42, 93-106.

Parra, J.O., and C.L. Hackert, 2002, Wave attenuation attributes as flow unit indicators: The Leading Edge, 21, 564-572.

Parra, J.O., and C.L. Hackert, 2006, Modeling and interpretation of $Q$ logs in carbonate rock using a double-porosity model and well logs: Journal of Applied Geophysics (in press).

Parra, J.O., C.L. Hackert, and M.W. Bennett, 2006, Permeability and porosity images based on P-wave surface seismic data: Application to a south Florida aquifer, Water Resources Research, 42, W02415, doi:10.1029/2005WR004114.

Parra, J.O., C.L. Hackert, H.A. Collier, and M. Bennett, 2001, A methodology to integrate magnetic resonance and acoustic measurements for reservoir characterization: U.S. Department of Energy National Petroleum Technology Office, DOE/BC/15203-3 (ID: 790861) (www.reservoirgeophysics.swri.edu).

Parra, J.O., C.L. Hackert, M. Bennett, and H.A. Collier, 2003, Permeability and porosity images based on NMR, sonic, and seismic reflectivity: Application to a carbonate aquifer: The Leading Edge, 22, 1102-1108.

Parra, J.O., C.L. Hackert, R. Green, and S. Pride, 2003, A double-porosity poroelastic model to relate P-wave attenuation to fluid flow in vuggy carbonate rock: Conference Proceedings, Royal Institute of Technology, Sweden. Editors: O. Stephansson, J. Hudson, and L. Jing, 481-486.

Parra, J.O., C.L. Hackert, and P.C. Xu, 2004, Q logs for lithology and pore structure discrimination. Presented at the SEG $74^{\text {th }}$ Annual International Meeting, October 10-15, Denver, CO; published in SEG Expanded Abstracts, 1754.

Parra, J.O., C.L. Hackert, and P.-C. Xu, 2006, Attenuation analysis of acoustic waveforms in a borehole intercepted by a sand-shale sequence reservoir: The Leading Edge, February, 187-193.

Parra, J.O., P.-C Xu, and C.L. Hackert, 2004, Algorithm to estimate $Q$ logs from full waveform sonic and its application to pore structure characterization in carbonates. Paper submitted to Geophysics.

Peterson, R.A., W.P. Fillipone, and F.B. Cohen, 1955, The synthesis of seismograms from well log data: Geophysics, 20, 510-530.

Pride, S., E. Tromeur, and J.G. Berryman, 2002, Biot slow-wave effects in stratified rocks: Geophysics, 67, 271-281.

Pride, S., and J.G. Berryman, 2002, Attenuation of P waves by wave-induced fluid flow, Proceedings, Second Biot Conference on Poromechanics, Auriault et al. (eds.), Grenoble, France, pp. 739-744. 
Pride, S.R., and J.G. Berryman, 2003a, Linear dynamics of double-porosity and dual permeability materials. I. Governing equations and acoustic attenuation: Physical Reviews, E68, 036603.

Pride, S.R., and J.G. Berryman, 2003b, Linear dynamics of double-porosity and dual permeability materials. II. Fluid transport equations: Physical Reviews, E68, 036604.

Pride, S., et al., 2003, Permeability dependence of seismic amplitudes: The Leading Edge, 22, 518-525.

Quan, Y., and J.M. Harris, 1995, Seismic attenuation tomography using the frequency shift method: Geophysics, 62, 895-905.

Quan, Y., J.M. Harris, and X. Chen, 1994, Acoustic attenuation logging using centroid frequency shift and amplitude ratio methods: A numerical study. Presented at the SEG $64^{\text {th }}$ Annual International Meeting, published in SEG Expanded Abstracts, 8-11.

Raikes, S.A., and R.E. White, 1984, Measurements of earth attenuation from downhole and surface seismic recordings: Geophysical Prospecting, 32, 892-919.

Rapoport, M.B., L.I. Rapoport, and V.I. Ryjkov, 2004, Direct detection of oil and gas fields based on seismic inelasticity effect: The Leading Edge, 23, 276-278.

Robertson, J.D., and H.H. Nogami, 1984, Complex seismic trace analysis of thin beds: Geophysics, 49, 344-352.

Sandersen, P.B.E., and F. Jorgensen, 2003, Buried quaternary valleys in western Denmark - occurrence and inferred implications for groundwater resource and vulnerability: Journal of Applied Geophysics, 53, 229-248.

Schmidt, H., and G. Tango, 1986, Efficient global matrix approach to the computation of synthetic seismograms: Geophysical Journal of the Royal Astronomical Society, 84, 331-359.

Sheriff, R.E., and L.P. Geldart, 1995, Exploration Seismology, $2^{\text {nd }}$ ed.: Cambridge Univ. Press.

Stockwell, Jr., J.W., 1999, The CWP/SU: Seismic Un*x Package: Computers and Geosciences, 25, 415-419.

Sumanovac, F., and M. Weisser, 2001, Evaluation of resistivity and seismic methods for hydrogeological mapping in karst terrains: Journal of Applied Geophysics, 47, 13-28.

Sun, X., X. Tang, C.H. Cheng, and L.N. Fraser, 2000, P- and $S$-wave attenuation logs from monopole sonic data: Geophysics, 65, 755-765.

Taner, M.T., and S. Treitel, 2003, A robust method for $Q$ estimation, SEG $73^{\text {rd }}$ Annual International Meeting, Expanded Abstracts, paper IT3.6.

Toksoz, M.N., D.H. Johnson, and A. Timur, 1979, Attenuation of seismic waves in dry and saturated rocks, 1, Laboratory experiments: Geophysics, 44, 681-690.

Trorey, A.W., 1970, A simple theory for seismic diffractions: Geophysics, 35, 762-784.

Trorey, A.W., 1977, Diffractions for arbitrary source-receiver locations, Geophysics, 42, 1177-1182.

White, R.E., 1992, The accuracy of estimating $Q$ from seismic data: Geophysics, 57, 1508-1511.

Wuenschel, P.C., 1960, Seismogram synthesis including multiples and transmission coefficients: Geophysics, 25, 106-129. 
Yamamoto, T., 2003, Imaging permeability structure within the highly permeable carbonate earth: Inverse theory and experiment: Geophysics, 68, 1189-1201.

Yilmaz, O., 2000, Seismic Data Analysis, $2^{\text {nd }}$ ed., Society of Exploration Geophysicists, Tulsa, Oklahoma, USA, 2027 pages.

Zahradnik, J., O. Patrick, and J. Sochacki, 1994, Finite-difference schemes for elastic waves based on the integration approach: Geophysics, 59, 928-937.

Zhang, C., and T.J. Ulrych, 2002, Estimation of quality factors from CMP records: Geophysics, 67, 1542-1547. 